$$
\begin{aligned}
& \text { AN AFFECTIVE } \\
& \text { AWARENESS }
\end{aligned}
$$




\title{
AN AFFECTIVE AWARENESS
}

\author{
By Bronwyn Phillipps
}

A thesis submitted to the Victoria University of Wellington in partial fulfilment of the requirements for the degree of Master of Architecture (Professional) 


\section{Acknowledgments}

Firstly, I wish to acknowledge my architecture class; for without your support, humour, knowledge, advice and friendship these last five years would have been unbearable.

To Natasha and Simon; your knowledge, direction and grounded approach to this year motivated me to work hard and not worry so much about the little things.

Mum and Dad, words cannot express the depth of my appreciation for your passion for learning, your life lessons, support and belief in my abilities even when I lacked it myself. To Papa, for constantly making me laugh with wild exaggerations about my architecture fame. And to my brother Ian for being my personal editor for the last five years.

To my flatmates over the years, thank you. Especially to Soph and Kir, for looking after me when low on sleep, energy and time. To Kayla, Brookey, Liv, Darnz, Mack, Kelly, Dybs, DB and all my other friends; thank you for providing me with endless humour, distraction and support.

Last but not least, to Jesse, thank you for always being there no matter where in the world you are, for listening, loving and believing in me. 



\begin{abstract}
One of the most captivating qualities of the New Zealand wilderness is its temporality; season's, weather, night and day, are multiple interconnected systems in flux. These temporal systems are intricately linked and determine the feel of the environment. We perceive this natural temporal environment through levels of experience that build from affects. Architecture is designed to resist this temporality, with lights, shelter, warmth and more. The limited engagement between interior and exterior also removes environmental conditions that have positive impact. The fixity of architecture, in its resistance to the uncontrollable, to the temporal flux inherent in the environment, limits the potential for wilderness architecture to relate to its constantly changing context.

This thesis argues that non-static architecture creates a stronger connection between people, architecture and the natural environment. The success of architecture to emphasise this connection is analysed through the lens of affect as a preconscious reaction, which counters the social and cultural expectations of space. The study of affect provided a framework of understanding to inform the active designing.
\end{abstract}

\begin{abstract}
A fluid semi-permanent shelter is designed, constructed of a woven structural system responsive to both weather conditions and the occupants. The activities that occur within these structures maximise the nonstatic potential for movement and create a constant interaction with the landscape. The interplay between uncontrollable flux and controllable movement in the structures provides a challenging context that heightens the inhabitant's awareness of the relationship between people, architecture and natural environment.
\end{abstract}




\section{Contents}

Abstract

Introduction

Problem

Aim + Proposition

Research Context + Scope

Wilderness

Affect

Non-static

Research Method + Thesis Structure

-1 -

11

12
Site

Ecology and Biology

Cultural History

Environmental History

Temporal Site Conditions

Geological Time

Weather/Seasonal Time

Short Time Period

Site Visits

Site Visit One - 17.02.13

Site Visit Two - 09.03.13

Site Visit Three - 31.04.13

Site Visit Four - 11.07.13

18

22

22

24

24

24

24

25

25

25

28

32

2

Context

Wilderness

38

A place for people, a place for design? 38 Affect

What is Affect? 40

Non-Static $\quad 42$

The Interactive Architecture Paradigm 42

Glamping 44

Precedents 46

Wilderness Case Study - Back Country Hut 46

Affect Case Studies - Weather Art + Architecture 52

Non-Static Case Studies - Movement Systems 56

Material Case Studies - Bamboo Architecture $\quad 58$

Material Case Studies - Skin Precedents 59

Structural Case Studies - Weave Precedents 59 
Phase One - Design Tests

Design Iteration $1 \mathrm{~A}$

A Wilderness Design Dialogue

Reflection

Design Iteration $1 B$

The Engineering of Affect through Design Affect Reflection

Design Iteration $1 C$

A Moving Interaction

Phase One Discussion

- 4 -

Phase Two - Concept Design

Space, Time and Affect

Design Iteration 2

Form - Structure

Sited Occupation

Concept One

Phase Two Reflection

$-5-$

$-7-$

Conclusion 
4 


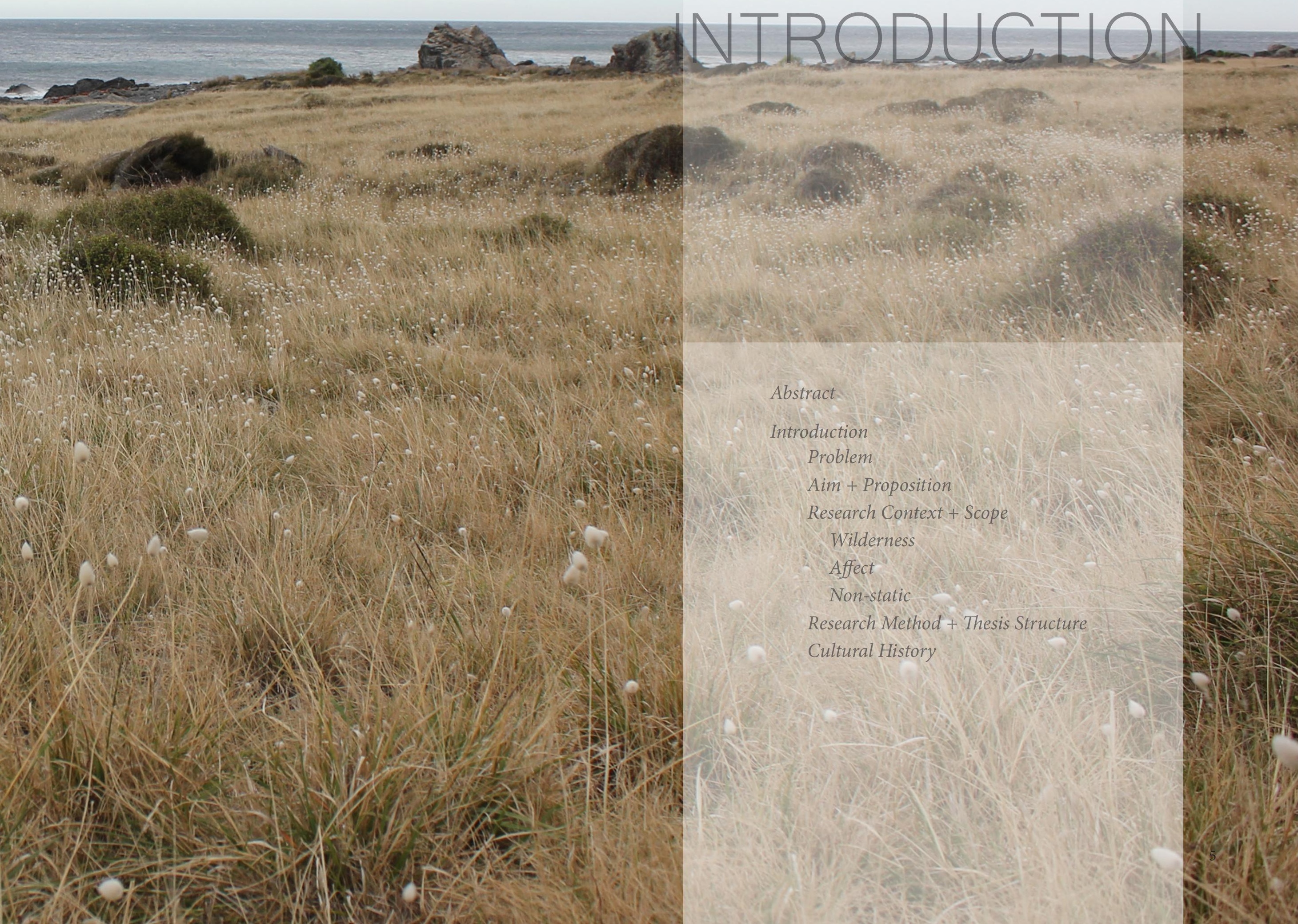




\section{Problem}

Traditionally architecture is designed to resist temporal conditions like; sun, rain, wind and snow. Limited engagement between interior and exterior removes environmental conditions that have positive impact. This resistance of the uncontrollable environment limits the potential for wilderness architecture to relate to its constantly changing context.

\section{Aim + Proposition}

Through design, this thesis aims to explore an interactive architecture that is adaptable to the temporality of its context. It also seeks to create a design that attracts people to a wilderness setting and amplifies a person's awareness and connection with the natural environment. This aim will be explored through the design of a semi-permanent glamorous camping shelter located in an accessible wilderness site on the southern-most tip of the North Island of New Zealand. A kinetic architecture is designed to challenge the preconceptions of architectural space by creating a built environment that is transformed by occupants and the surrounding environmental conditions. The balance between controllable and uncontrollable movement in the shelter creates a constantly indefinable relationship that creates connection with the landscape.

Introduction 


\section{Research Context + Scope}

The research context has been defined through three fields; wilderness, affect and non-static.

\section{Wilderness}

Wilderness is the physical context, the rich natural environment and a term that is commonly used to represent an iconic New Zealand landscape. Abbott (2004) discusses wilderness as a separation between social culture and the untouched, untainted natural environment. ${ }^{1}$ This disconnect establishes a challenging context for this research. In my view, there is a need to create a dialogue between social, cultural and natural fields to reintroduce our wilderness landscapes as places for people to inhabit.

\section{Affect}

The success of the shelter to emphasise a connection between wilderness environments and people is analysed through the lens of affect. Affect is a preconscious reaction, which counters the social and cultural expectations of space. There are multiple translations of affect but the scope of this investigation is limited to the relationships present in the Darwinian/ Spinozian approach to affect and the drive based affect theories. Both translations framed my design decisions. Tomkins, Sedgwick and Frank's critique of the Freudian singular drive approach provides a base understanding of the differences between survival and emotional or experiential drives. I have then foregrounded Nigel Thrifts theories, which align with the Darwinian/Spinozian tradition and provide art-based methods of increasing affect. ${ }^{2}$
Non-static

Non-static, interactive architecture is a field that is currently dominated by technological developments. Technology is used to create architectural spaces that respond to spatial requirements; energy consumption, programmatic or climatic changes. ${ }^{3}$ There is little research into the experiential qualities of non-static architecture and the value of interactive systems designed to heighten the occupant's awareness of space. When a building is static, the occupant can understand it from a conventional perspective. If we explore the notion of space as undeterminable and deformable, it can become less defined in the mind of the occupant, thus creates the possibility for repetitive moments of surprise and connection with each encounter ${ }^{4}$. 


\section{Research Method + Thesis Structure}

This thesis engages physical and digital modelling as a method. The design process is split into three phases follow the development of the shelter, refer to Figure 0.1.

Each phase is examined through a three-layer method:

Investigation: Theoretical and visual framing occurs at the beginning of each design phase. This refers to one or multiple ideas from the context established in section two that will be explored through design.

Design: Investigates form, structure, occupation, programme and relationship to site.

Reflection: Critically reflects on each section with indication of how this informs the next stage of research.

A thorough site description and analysis is explained through visual, written and film media in section one. Following this, Chapter Two positions the research within theoretical, art and architecture contexts defined through case studies and literature follows this. In section three we begin the first design phase with design research tests into wilderness, affect and nonstatic. Phase Two explains the development of the structural mechanism and describes the design process for concept one. Phase Three is the developed design phase where I design through digital and analogue models to resolve the structure, followed by defined narrative and programme development. This phase is concluded with a detailed section that resolves the shelter to a product level. A final discussion and conclusion summarises the process and findings.

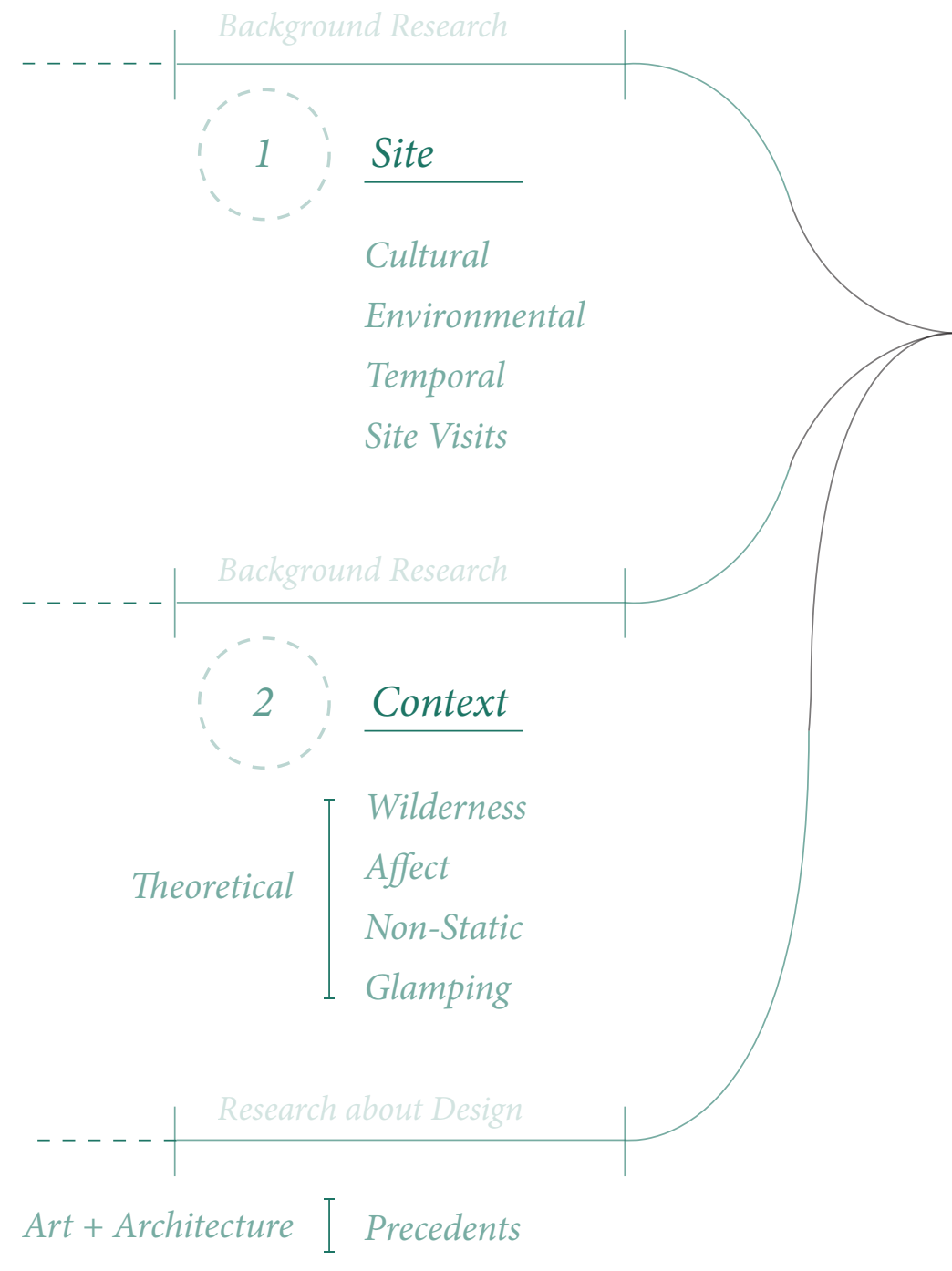

Figure 0.1

Visual Diagram of the thesis structure/research process 


\section{Phase One Design Tests}

$1 \mathrm{~A}$ Investigation

Design

Reflection

$1 B$

Investigation

Design

Reflection

1C Investigation

Design

Reflection

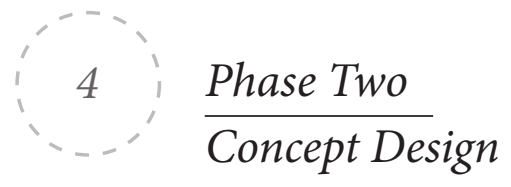

$2 A$

Investigation

Design

Reflection

\section{Phase Three \\ Developed Design}

$3 A$

Investigation

Design

Reflection

1. Mick Abbott, "Designing Wilderness as a Phenomenological Landscape: Design-Directed Research Within the Context of New Zealand's Conservation Estate" (Lincoln University, 2008), 12, http://researcharchive. lincoln.ac.nz/dspace/handle/10182/1026.

2. Nigel Thrift, "Intensities of Feeling: Towards a Spatial Politics of Affect," Geografiska Annaler: Series B, Human Geography 86, no. 1 (2004): 57-78, doi:10.1111/j.0435-3684.2004.00154.x.

3. Michael Fox and Miles Kemp, Interactive Architecture (Princeton Architectural Press, 2009), 20.

4. Mehul Bhatt, Frank Dylla, and Joana Hois, "Spatia-Terminological Inference for the Design of Ambient Environments," in Spatial Information Theory (Aber Wrac'h, France: COSIT, 2009), 81.France: COSIT, 2009 
${ }^{10} \mid$ 


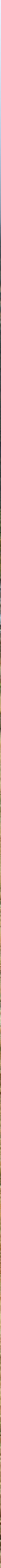


The site selected for this proposal lies on the South Wairarapa Coast at the southern-most tip of the North Island (Figure 1.1). This shelter is to be situated within Ngā Pōtiki Reserve, a Department of Conservation block located two and a half hours drive from Wellington City, just north of Ngawi and Matakita-ki-a-Kupe (Cape Palliser). As you pass Cape Palliser lighthouse the sealed road turns into a rough four-wheel drive track that winds through rugged private farmland until you reach the reserve. Strong northerly and southerly storms batter the site as winds rush along the hills towards the reserve and the open expanse of tussock lands on either side of Waitutuma stream. Trees and shrubs are sculpted to form large solid expanses of dense growth that ascend the rocky scree fan. The Aorangi Range towers over the lowland expanses as the site rapidly rises from sea level to $856 \mathrm{~m}$ (Mangatoetoe) around 300-400m inland from shore (Figure 1.2). This wilderness site is ruled by the elements and conditions on site are temporal in the extreme due to immense scale of the hills that funnel wind and attract rain.

\section{Environmental History}

The Wairarapa region consists of three different environmental zones - a mountainous hinterland (the Aorangi Mountains), a complex alluvial valley (Wairarapa Plains) with associated rivers and lakes, and a narrow coastal platform". 'The Aorangi Mountains and narrow coastal platform are clear features of the Ngā Pōtiki Reserve block as well as the Ngā Pōtiki Fan; a large redeposited gravel that extends out of a river valley into a forested shingle scree (refer to Figure 1.2, 1.3). Further up the coast the screes descend all the way to the ocean and form impressive expansive landscapes.

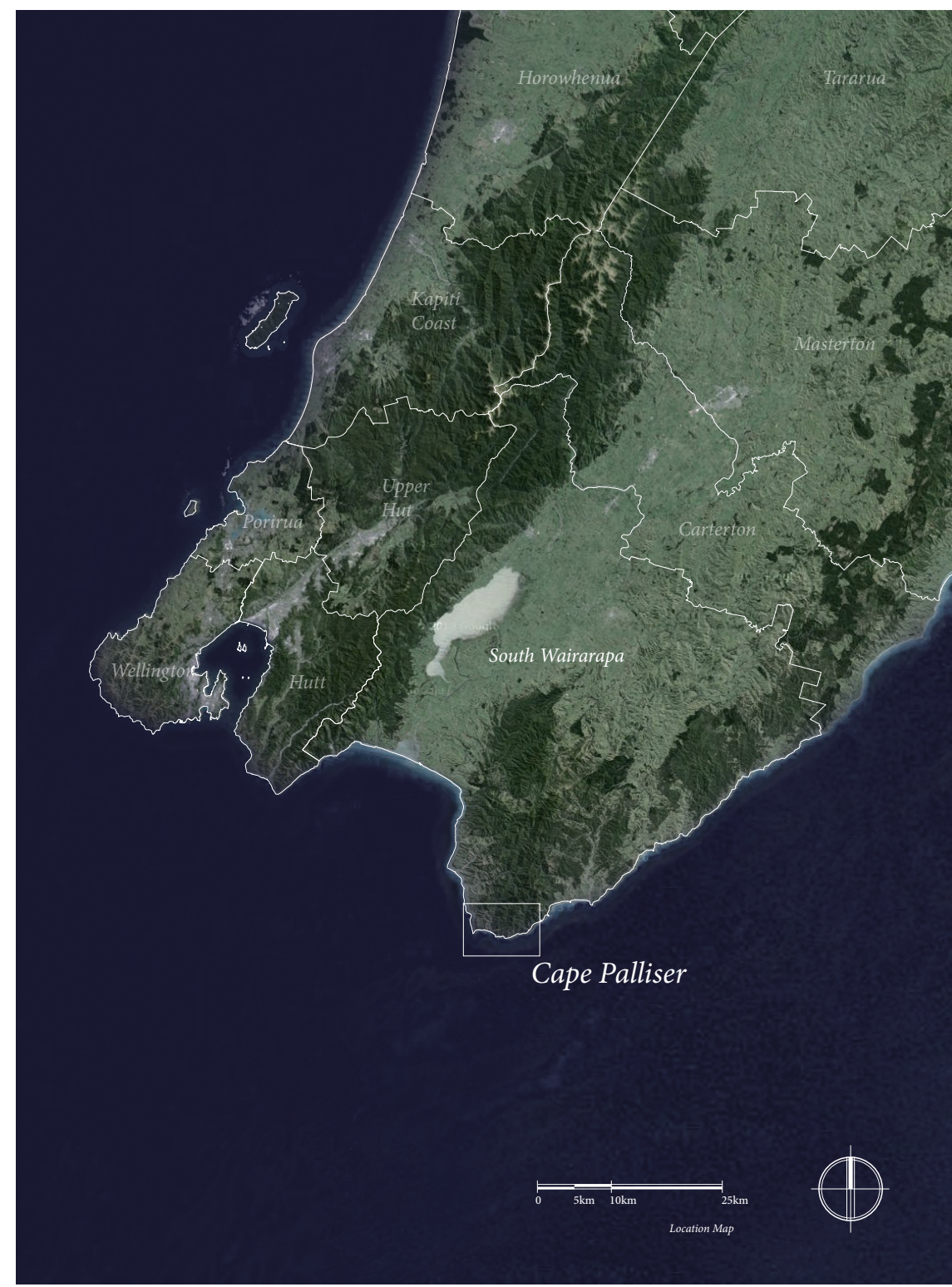

Figure 1.1

Site Map, showing Ngapotiki reserve in relation to the lower north island.

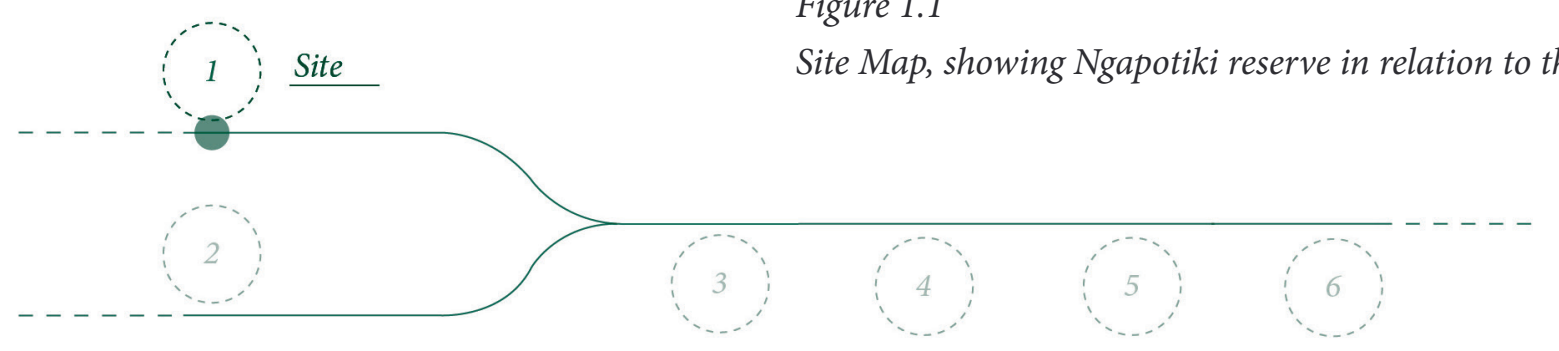




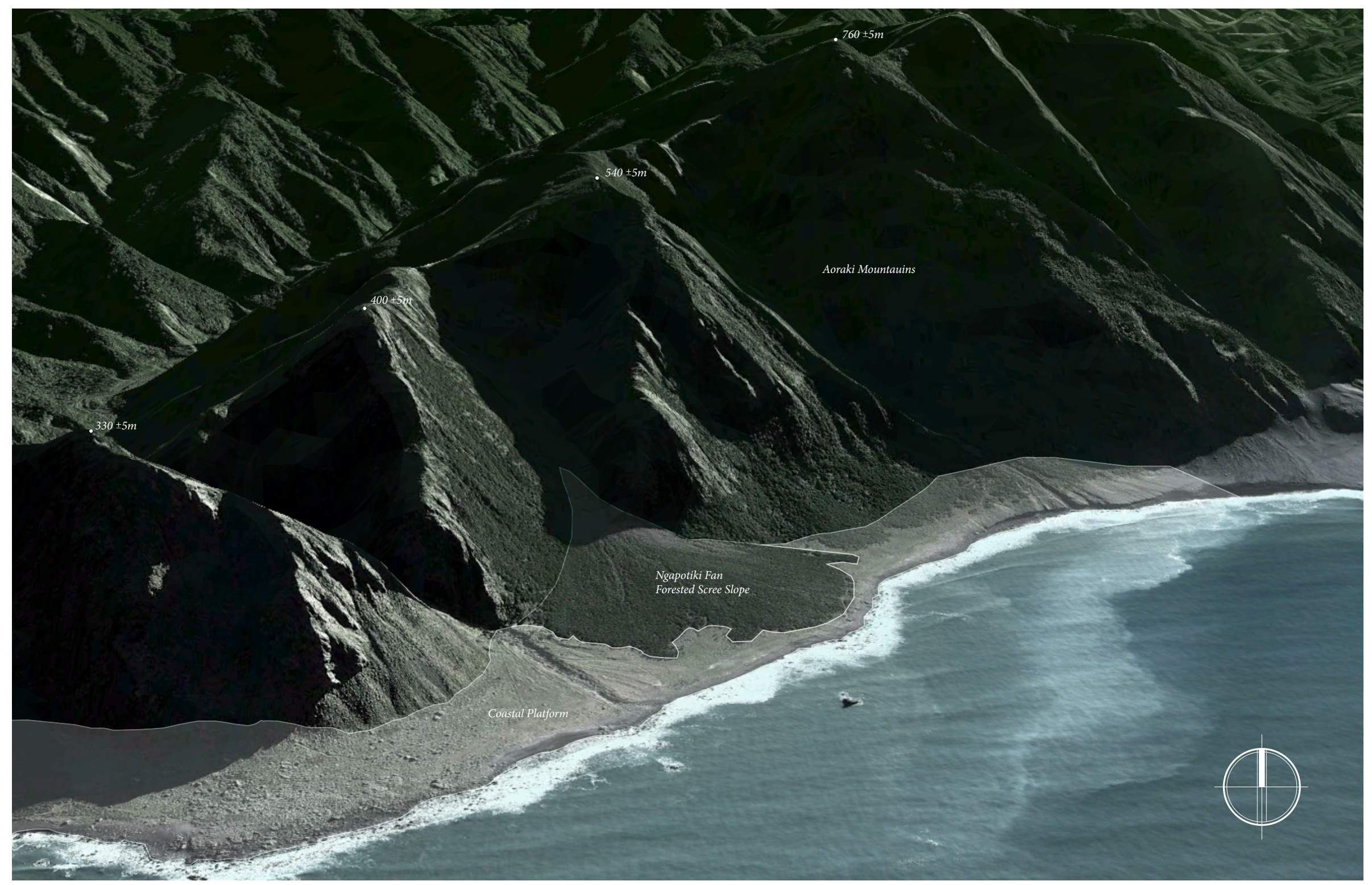

Figure 1.2

Aerial perspective of site showing the scale of the landscape, as well as the two of the three different environmental zones.
Figure 1.3 (following page)

Site Map, 1:2500 


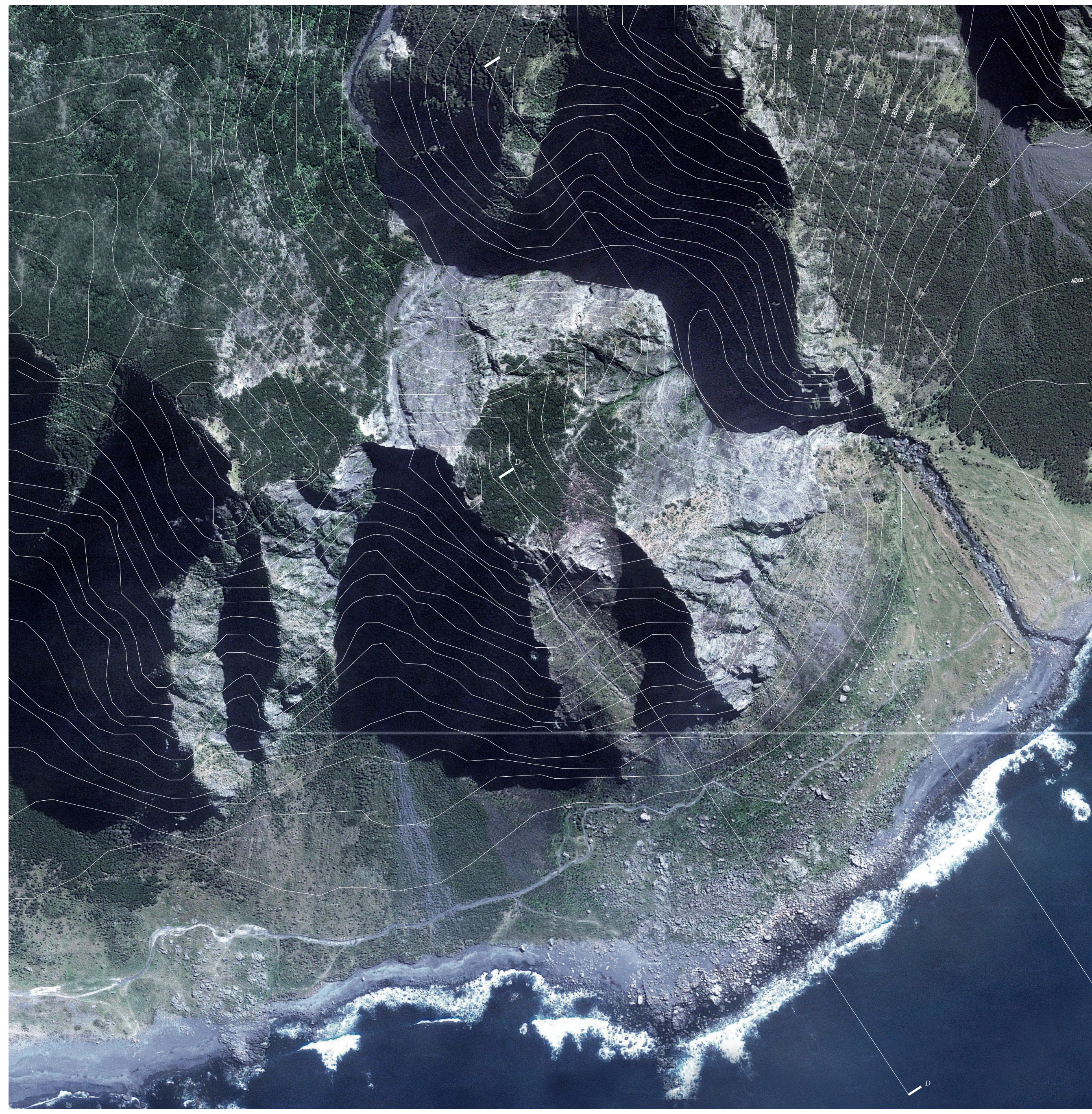




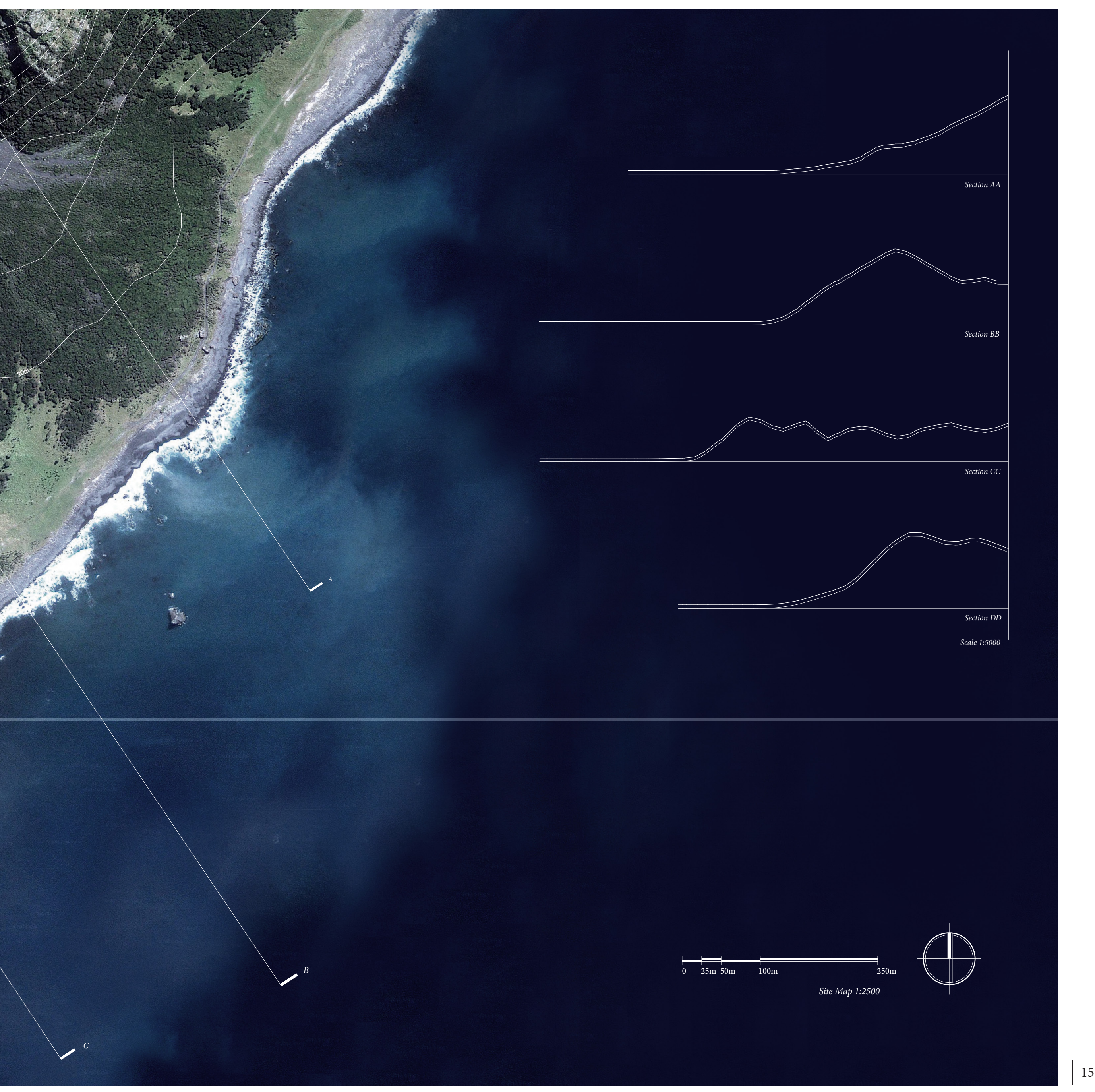


In the study of "Indigenous Vascular Plants in the Vicinity of Cape Palliser, Southern Wairarapa" a number of Dicot trees were recorded to grow onsite. ${ }^{2}$ A comparative analysis of the properties of these trees was undertaken to identify timber materials that could be sourced locally, ideally not treated, and would have a natural durability to withstand the exposed conditions at Ngā Pōtiki Reserve. ${ }^{3}$

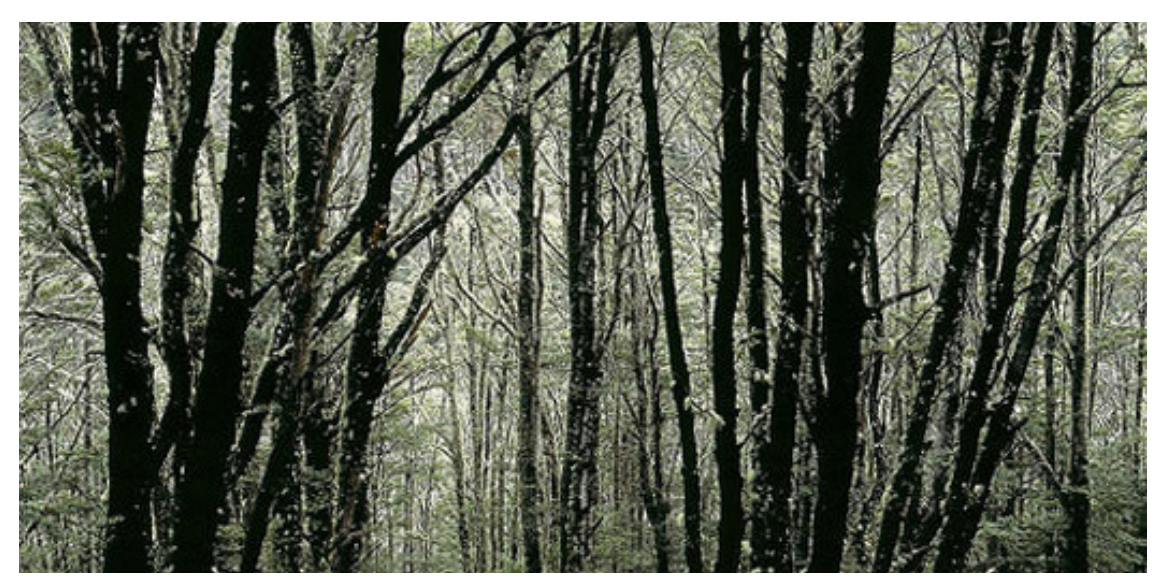

Black Beech - Nothofagus solandri

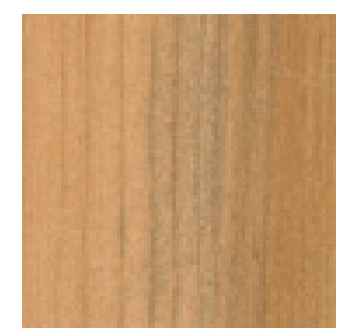

This is a strong and stable timber with variable durability properties. Both the heartwood and sapwood will rot in high hazard conditions. ${ }^{4}$ This makes it inappropriate for exposed use but a potential option for cabinetry due to its appealing red, brown and blackish colour.

Density: The air-dry density ranges from $650-720 \mathrm{~kg} / \mathrm{m}^{3}$

Durability: $50 x 50 \mathrm{~mm}$ posts last 10-15 years in ground

Shrinkage: $6 \%$ moisture content from green has $12 \%$ shrinkage in tangential direction and $4 \%$ radially

Hazard Class Rating: H3.1 ${ }^{5}$

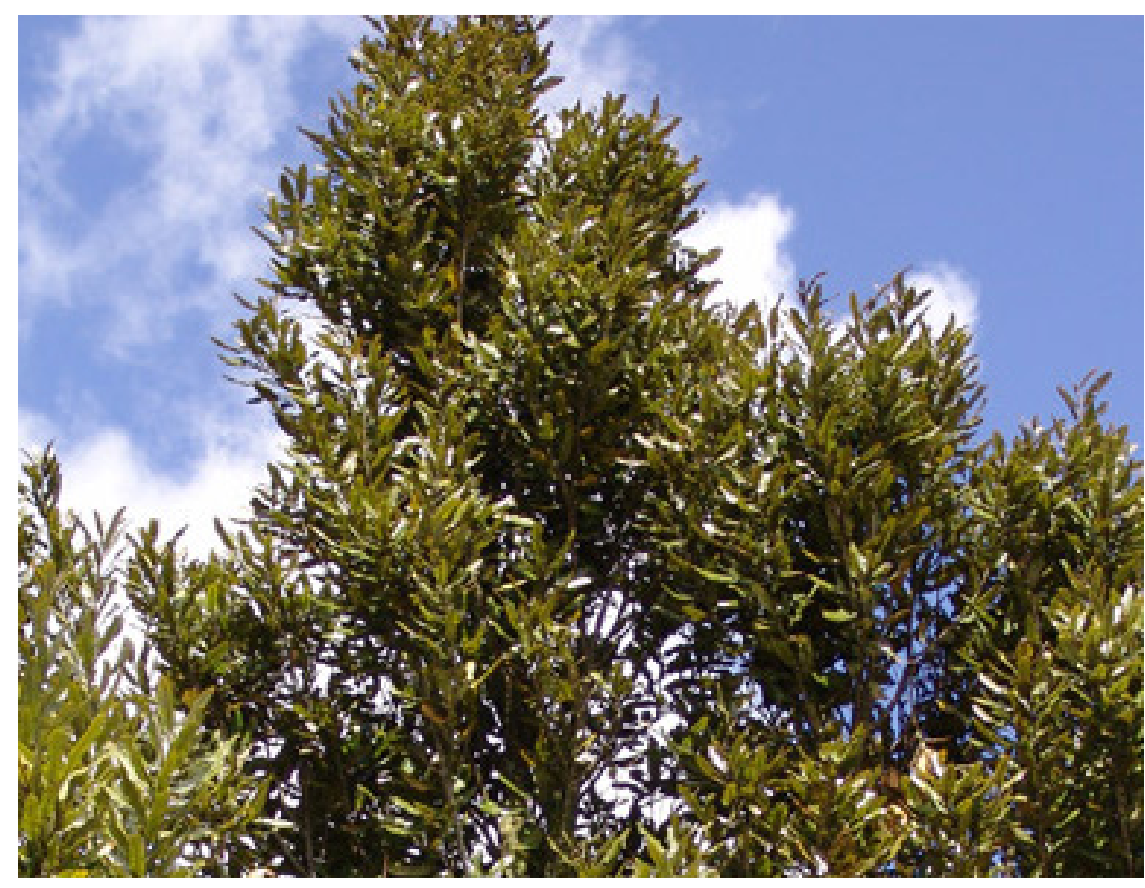

Rewarewa - Knightia excelsia

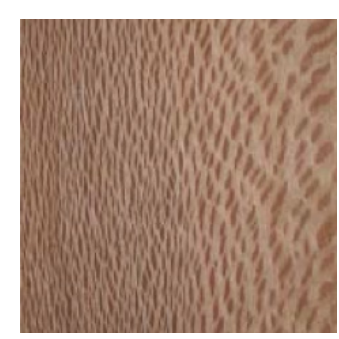

This timber is best known for its unique speckled appearance and use in internal cabinetry. Although strong, tough and easy to machine the heartwood is non-durable and the sapwood is susceptible to borer attack. Positively this wood has low susceptibility to fire and excellent acoustic properties. ${ }^{6}$

Density: $740 \mathrm{~kg} / \mathrm{m3}$

Strength: very strong modulus of rupture of $125 \mathrm{MPa}$, very stiff modulus of elasticity of $18.3 \mathrm{GPa}$

Durability: non-durable in ground

Shrinkage: from green to $12 \%$ moisture content, tangential shrinkage $10.2 \%$, radial $4.1 \%{ }^{7}$ 


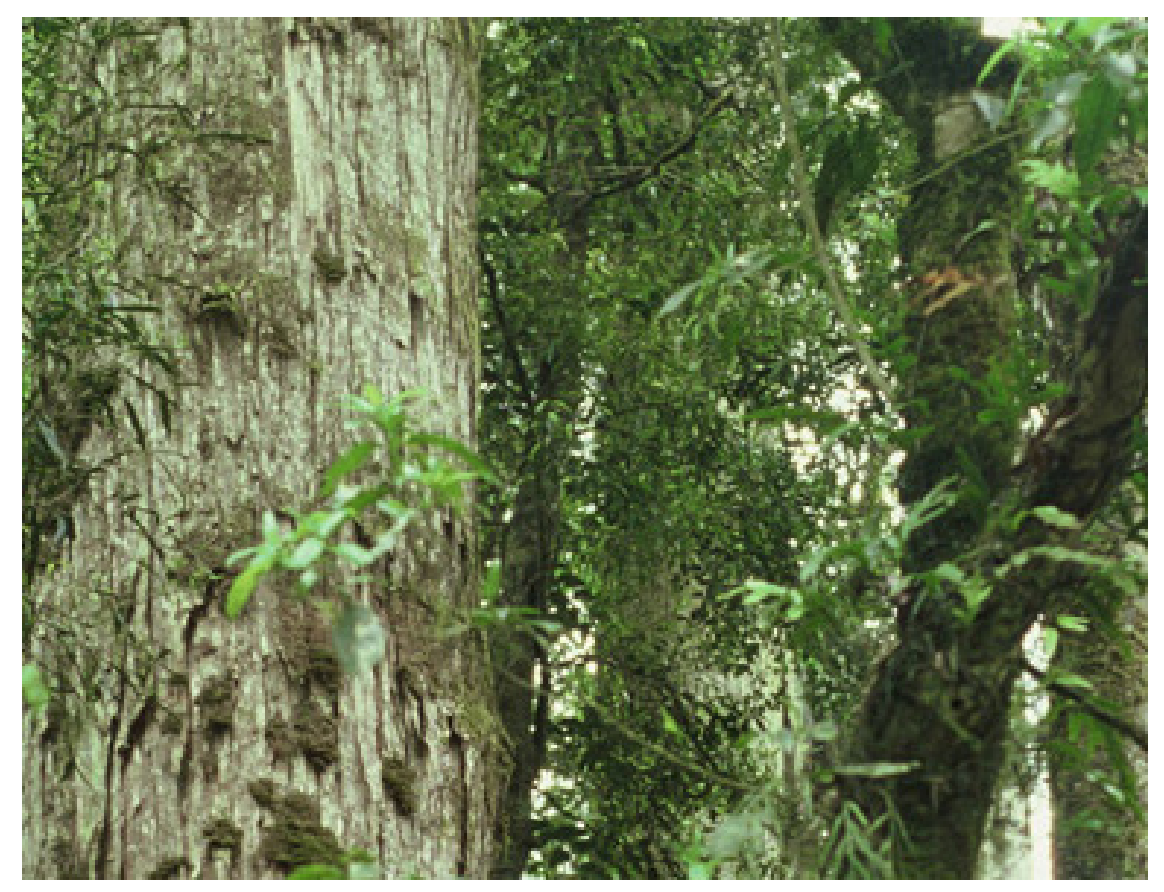

Totara - Podocarpus totara

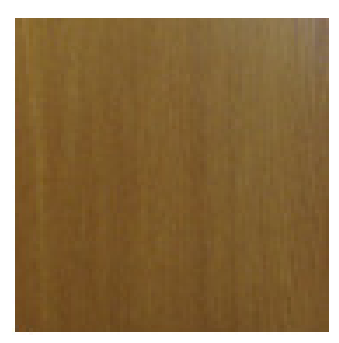

Although this species of tree is not present on site it historically grew in similar lowland areas up to $600 \mathrm{~m}$ altitude. ${ }^{8}$ Despite the timber being soft it is a light, extremely stable and durable wood with good compression strength. Salvage Totara heartwood is a viable option for compression members of the design.

Density: $560-580 \mathrm{~kg} / \mathrm{m} 3$

Strength: neither strong with modulus of rupture of $62 \mathrm{MPa}$, or stiff with modulus of elasticity of just 6.4Gpa. Performs in compression with compression strength parallel to grain of $41.8 \mathrm{MPa}$

Durability: a $50 \times 50 \mathrm{~mm}$ stake would last 25 years in ground.

Shrinkage: green to $12 \%$ moisture content has tangential shrinkage of $4.0 \%$, radial shrinkage of $2.0 \%$.

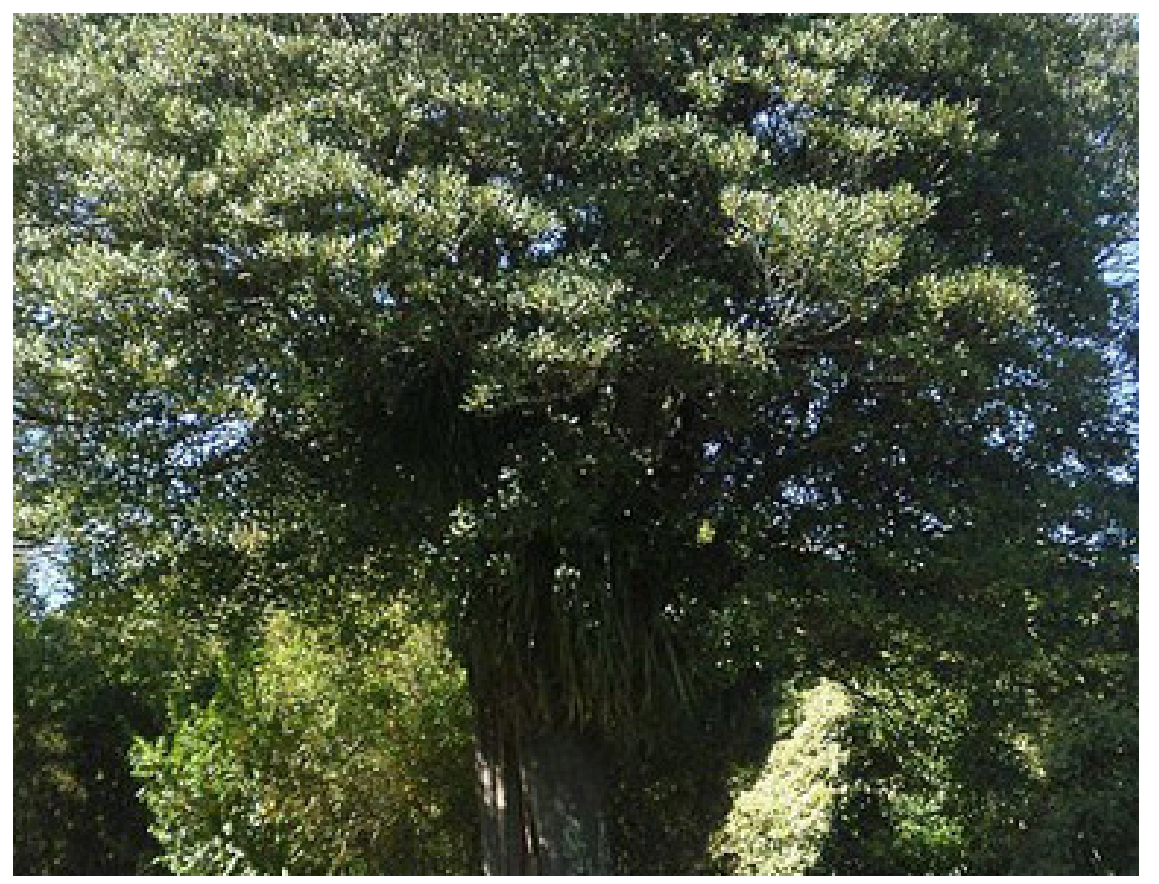

Hinau - Elaeocarpus dentatus

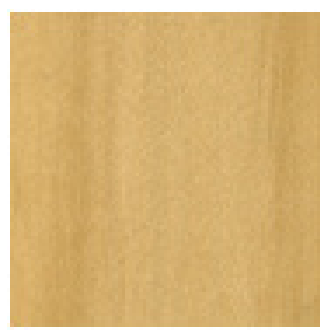

Hinau is a rare timber to find supplies for construction. It also has a low susceptibility to fire and can be preservative treated. It was used traditionally by the Maori tribes for the tannin (dye) in its bark, and the timber was used by European settlers for houses, bridges and highgrade hinau was used for boat building.

Density: $705 \mathrm{~km} / \mathrm{m} 3$

Strength: modulus of elasticity of $10.4 \mathrm{GPa}$ and a modulus of rupture of 89 $\mathrm{MPa}$

Durability: black heart hinau lasts 15-25 years in ground (by experience not scientific study), white heart hinau lasts only 5 years

Shrinkage: green to $12 \%$ moisture content gives tangential shrinkage of $7.0 \%$, radial of $2.6 \%$. 


\section{Cultural History}

The site has a rich Maori history with archaeological evidence of inhabitation from as early as the $12^{\text {th }}$ century. ${ }^{8}$ There is evidence of both permanent and shifting settlements as tribes retreated to semi-permanent camps inland to avoid bitter winter conditions. ${ }^{9}$ In $1250 \mathrm{AD}$ the indigenous population hit maximum land capacity of 300 people. The Ngāti Hinewaka tribe still regards Mātakitaki-a-Kupe (Cape Palliser) as wāhi tapu 'a sacred place,' despite abandonment in the $16^{\text {th }}$ century due to a mini ice age that altered the climatic conditions at the site and made it difficult to cultivate crops. ${ }^{10}$

After a period of absentee ownership, European settlement occurred in 1846 when the Cape Palliser block was initially leased, then freeholded. The New Zealand Government allowed run holders to purchase large expanses of land in 1853. ${ }^{11}$ These areas were pastoralised and grazed with sheep and cattle. The Ngā Pōtiki block was renamed White Rock Station and leased by a succession of farmers including Billy Barton who constructed rock walls to mark the boundaries of land; these are still visible on the site today. ${ }^{12}$ The land is still being farmed but was passed into Department of Conservation hands and is now a public reserve despite Ngäti Hinewaka having ownership of the coastal strip used for access.

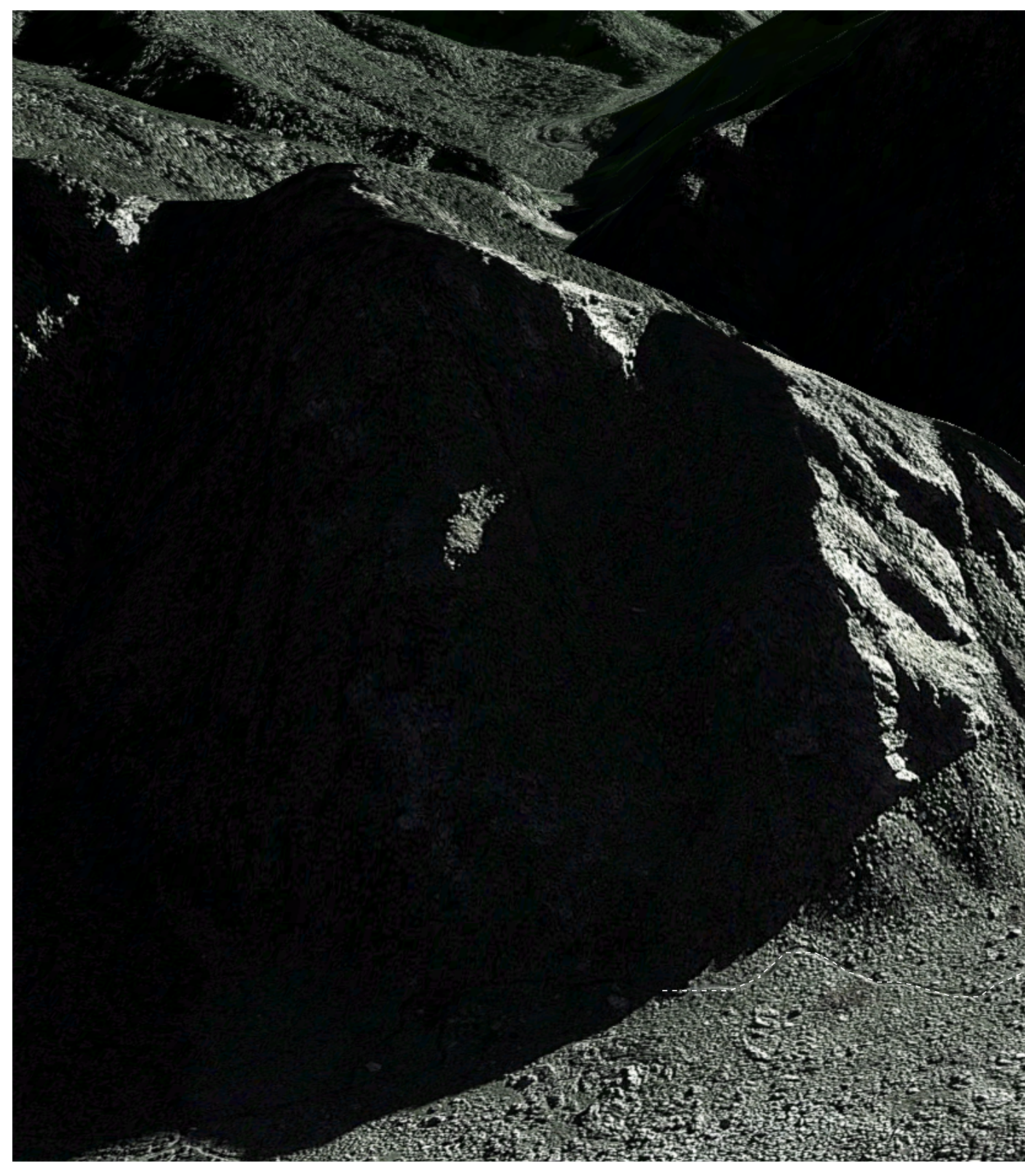

Figure 1.4

Potential, showing cultural history of site, locations of kumara beds, stone walls, and other more recent layers of cultural history. 


\section{Temporal Site Conditions}

"Human and animal activity peppers the landscape with points of movement"

Temporality exhibits itself through two interconnected conditions of this landscape; firstly the scales of permanence and change of the landscape as a whole and secondly the active movement present at specific points on site.

\section{Geological Time}

The terrain is modified through geological time. This forms the most permanent elements of this coastline, with slow periods of change in intervals of about 2500 years. The most recent of these was in 1855 when a magnitude 8.2 earthquake struck the region and caused significant movement along the Cape Palliser fault. The coastline was altered through raised terraces and substantial rock debris, as can be seen on Ngā Pōtiki Reserve today (Figure 1.5).

\section{Weather/Seasons}

Changing seasons operate over a shorter time-base, slowly altering the vegetation of the landscape. The weather conditions are dominated by long hot windy droughts in the summer with prevailing northwesterly winds and wild storms in the winter with southerlies bringing in icy blasts. ${ }^{13}$ It is a temperate environment with a range of $7-19^{\circ} \mathrm{C}$ between winter and summer. ${ }^{14}$

\section{Fleeting Time}

Human and animal movement, and changing weather systems interact with the landscape constantly through points of contact. This rapid temporal movement is one of the sites defining features, which constantly modifies the experience of landscape.

Bill Dolson reinforces the influence that seasons and weather have over the temporality of the environment and their critical relationship to movement. ${ }^{15}$ The site was analysed through a series of visits. This analysis is displayed in the following site visit sections.

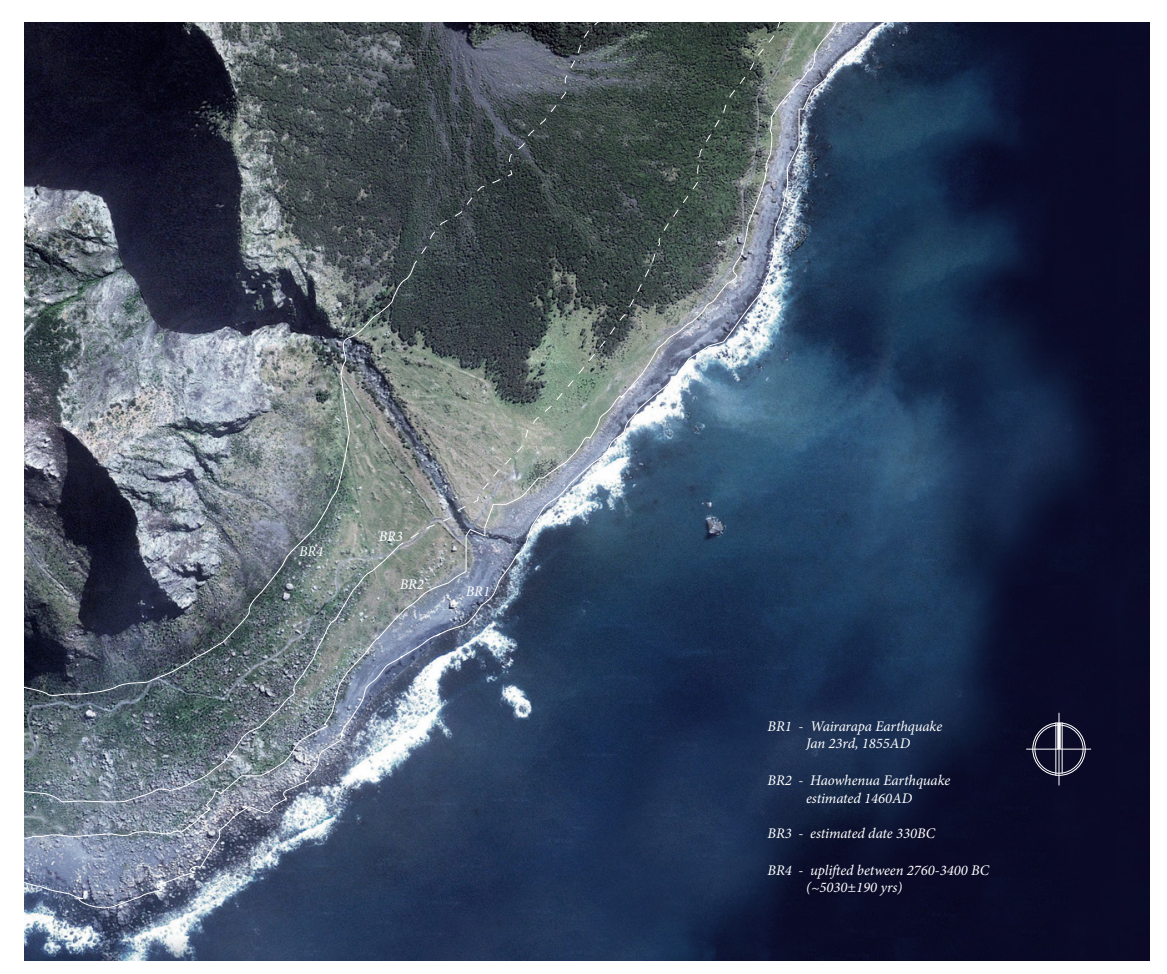

Figure 1.5

Seismic movement visible on site. 


\section{Site Visits}

Site Visit 1 - 17.02.13

Initially I sought to explore the coastline and get a feel for the atmosphere of the space. I drove to the site along the rough four-wheel drive track and walked when we could go no further. On this trip I did not reach Ngā Pōtiki Reserve due to a lack of time but explored the beaches and took photos of the journey towards site. This trip identified movement and temporality as key characteristics of this coast

\section{Site Visit 2 - 09.03.13}

A second trip was made to explore the reserve and to identify different temporal and atmospheric conditions. Five main areas were identified which included:

- Steep rocky hills

- Flat rocky grasslands

- Manuka/Kanuka forested scree fan

- Waterfall and streambed area

- Dense Manuka/Kanuka hillside

Movement was filmed at multiple locations within the reserve to investigate motion, oscillation, period of movement, type of movement and natural rhythm. This footage was later analysed through a process of drawing and modelling to create non-static architectural forms (explained in Chapter Three, Design Tests).

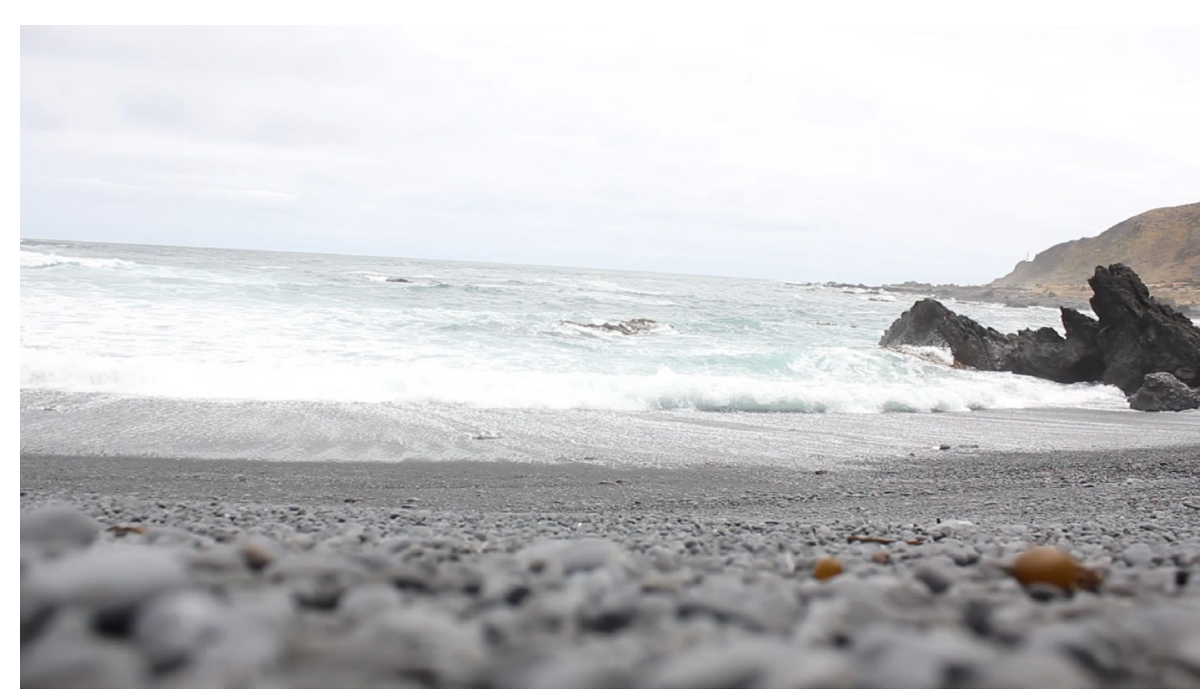

Click image to play video
Figure 1.6

Video footage of wave movement on site was recorded to later analyse the time period between waves and the changing forms. 

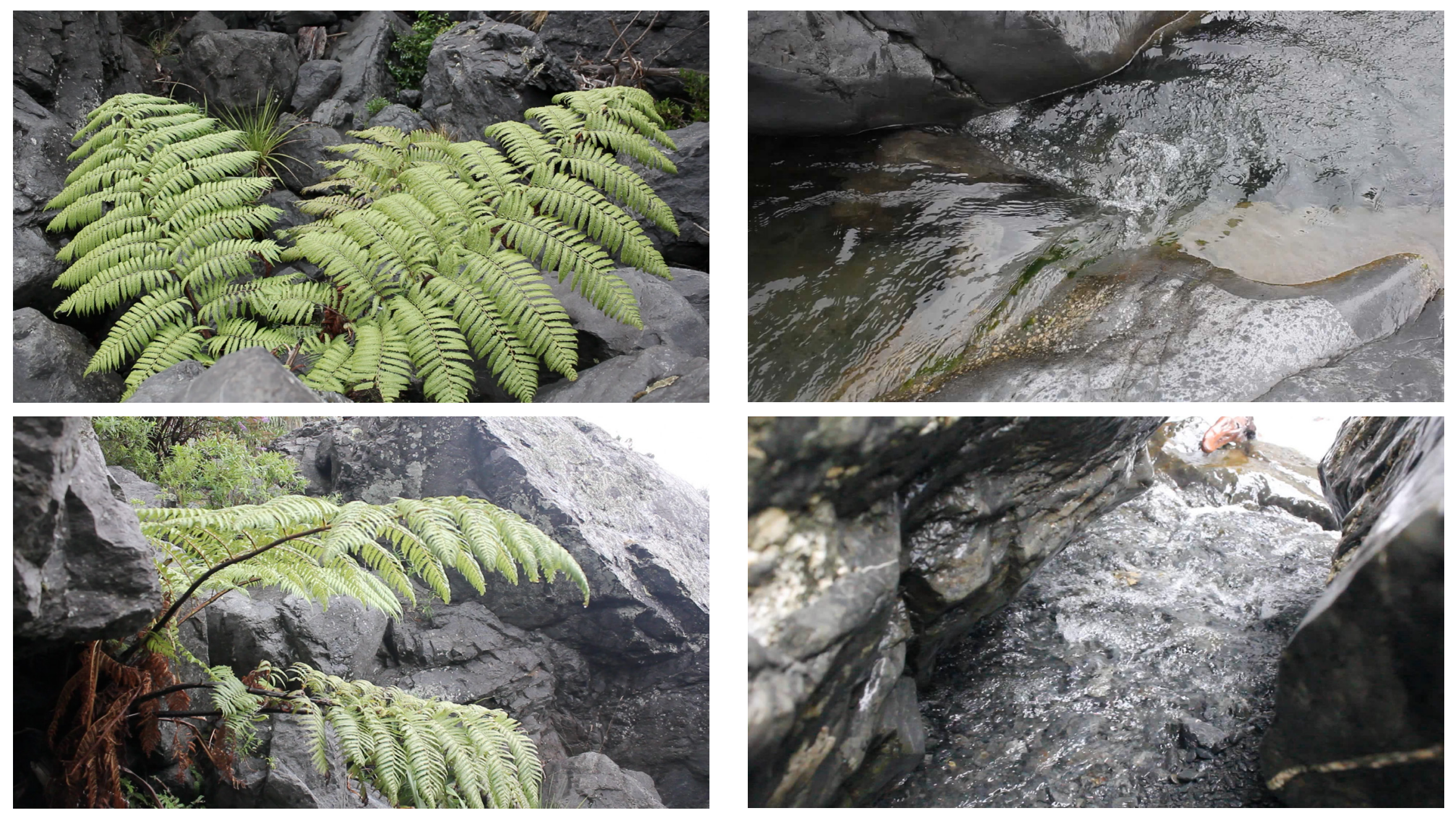

Click all images to play videos

Figure 1.7

Video footage of fern frond movement as wind gusts interact with the leaves. This was also analysed for periods of natural rhythm and motion.
Figure 1.8

Water movement in Waitutuma stream. Both videos show constant movement with little to distinguish periods of natural rhythm. 


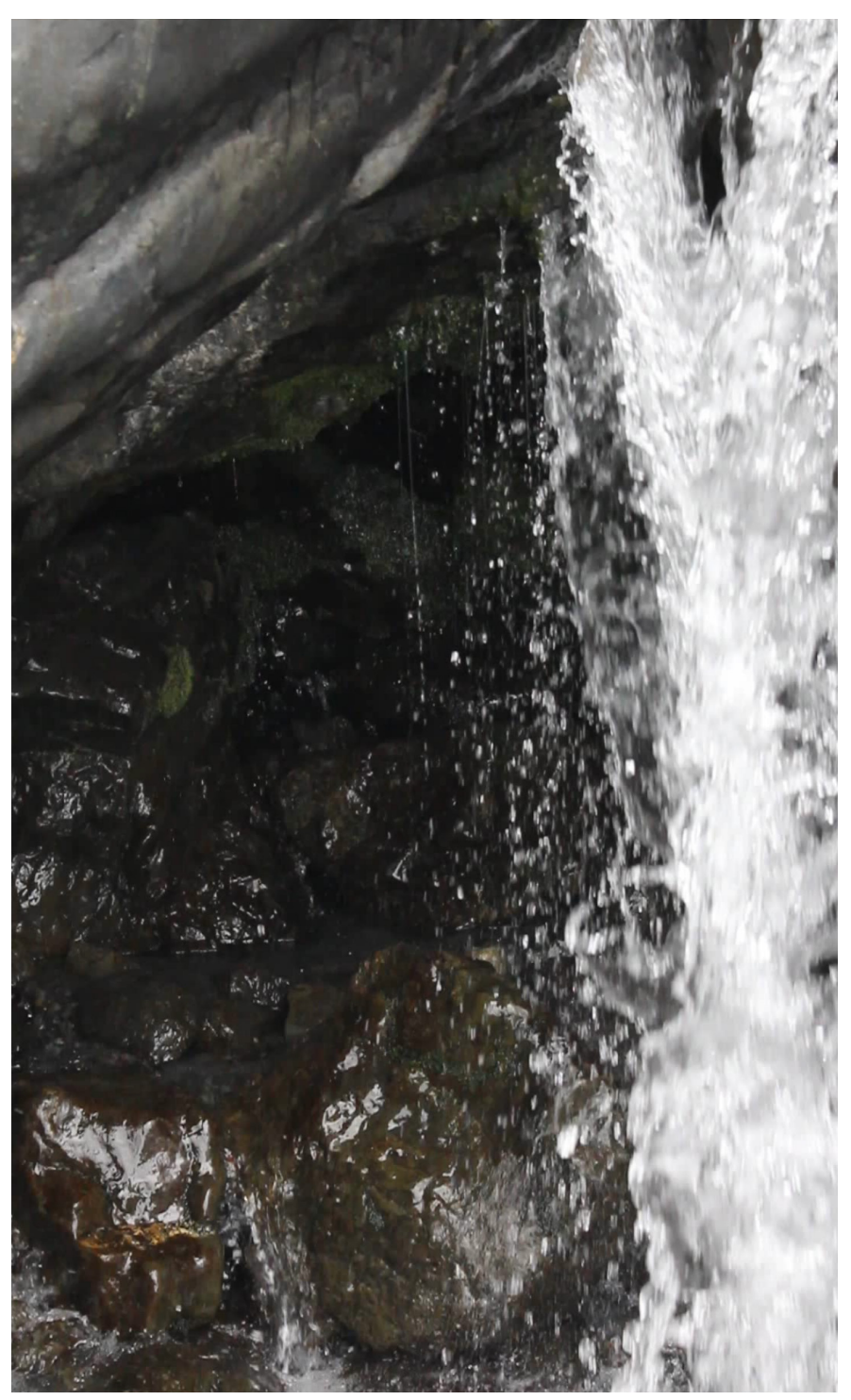

Click image to play video

Figure 1.9

Further footage of water movement in Waitutuma Stream. This was

recorded to show the contrast between drip path movement and waterfall.

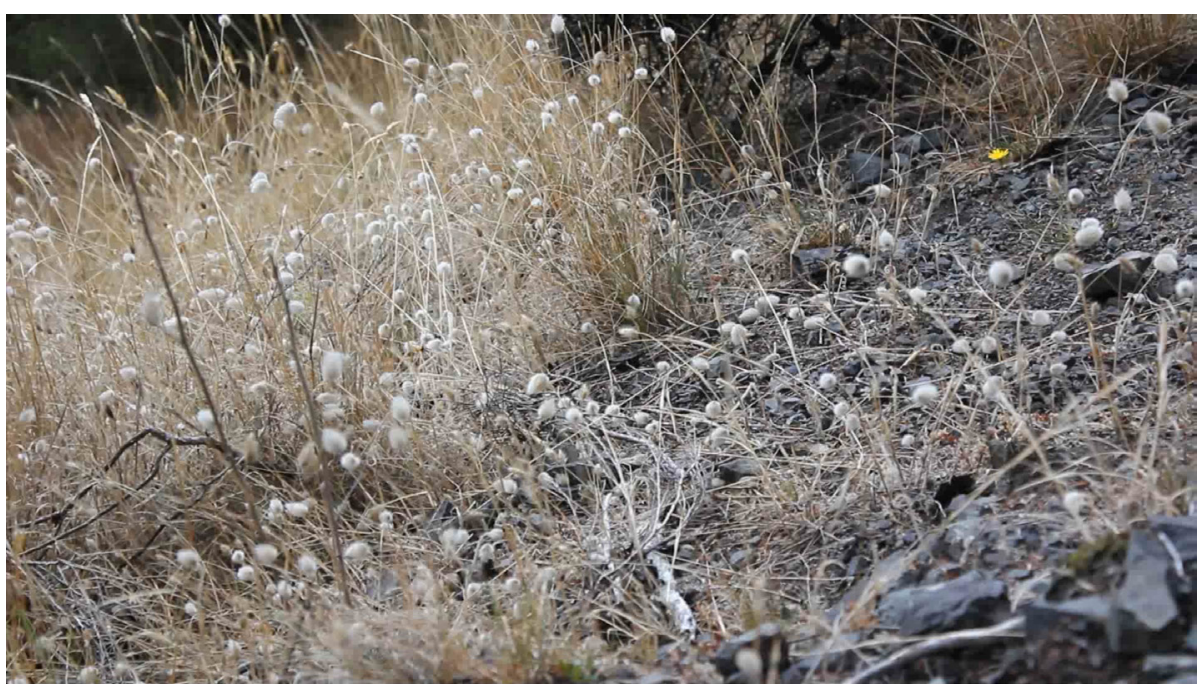

Click image to play video

Figure 1.10

Movement of Lagurus ovatus, Poaceae grasses in the wind. 
For site visit three, I camped at the reserve overnight to simulate the experience of staying overnight and to create a narrative for programme development. It also gave me a chance to identify some specific locations to site the shelter. The winds that night were described as moderate gusts by the weather forecast, but were so strong they shattered a fibreglass tent pole and rendered the tent unusable. I was forced to remove the fly and sleep outside on ground mats. This ended up more pleasant as there was no rain and it reduced the noise from the wind battering the tent. The most interesting thing about this experience was hearing the way the wind rushed around the mountains from the south, before hitting us. The hills create a barrier that causes a wind tunnelling effect. Consequently, at this stage of the design process the focus turned to practical constraints such as wind loading.

\section{Manuka / Kanuka Site}

This site visit identified the general area of densely forested scree as the site for the shelter. This was an area of protection, seclusion and yet movement, displayed through the angled forms of the gnarled trees (refer to Figure 1.11). The Manuka and Kanuka trees provide a natural canopy and grow densely up the scree slope to allow subtle inhabitation of the landscape. Navigation through this space is difficult due to the uneven ground, branches and close-knit trees (refer to Figure 1.13). This creates points of contact between the person and the landscape that become memorable and necessary in such a visually dense landscape.

Figure 1.11 (right)

Manuka/Kanuka scree site plan, showing stream, 1:250.

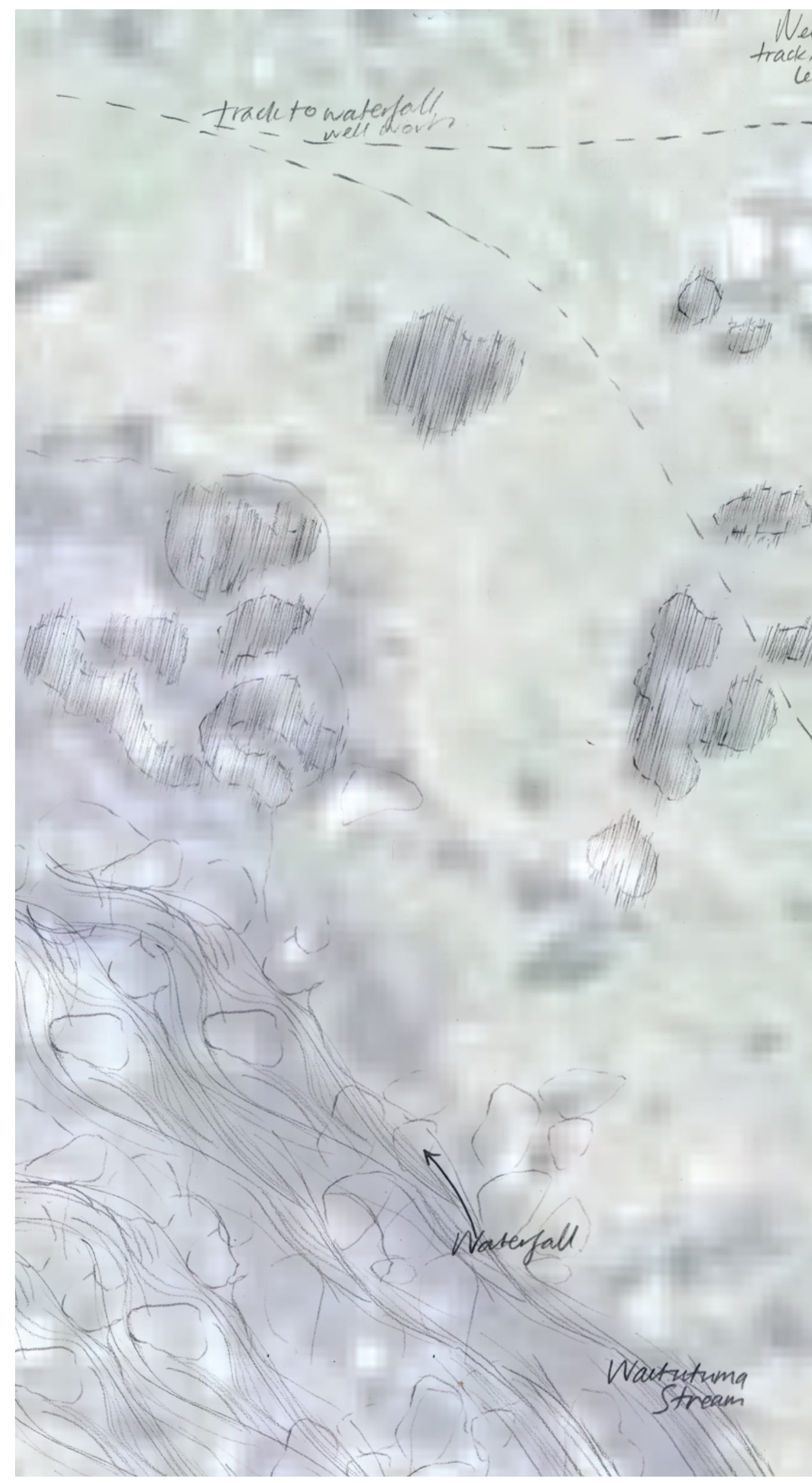




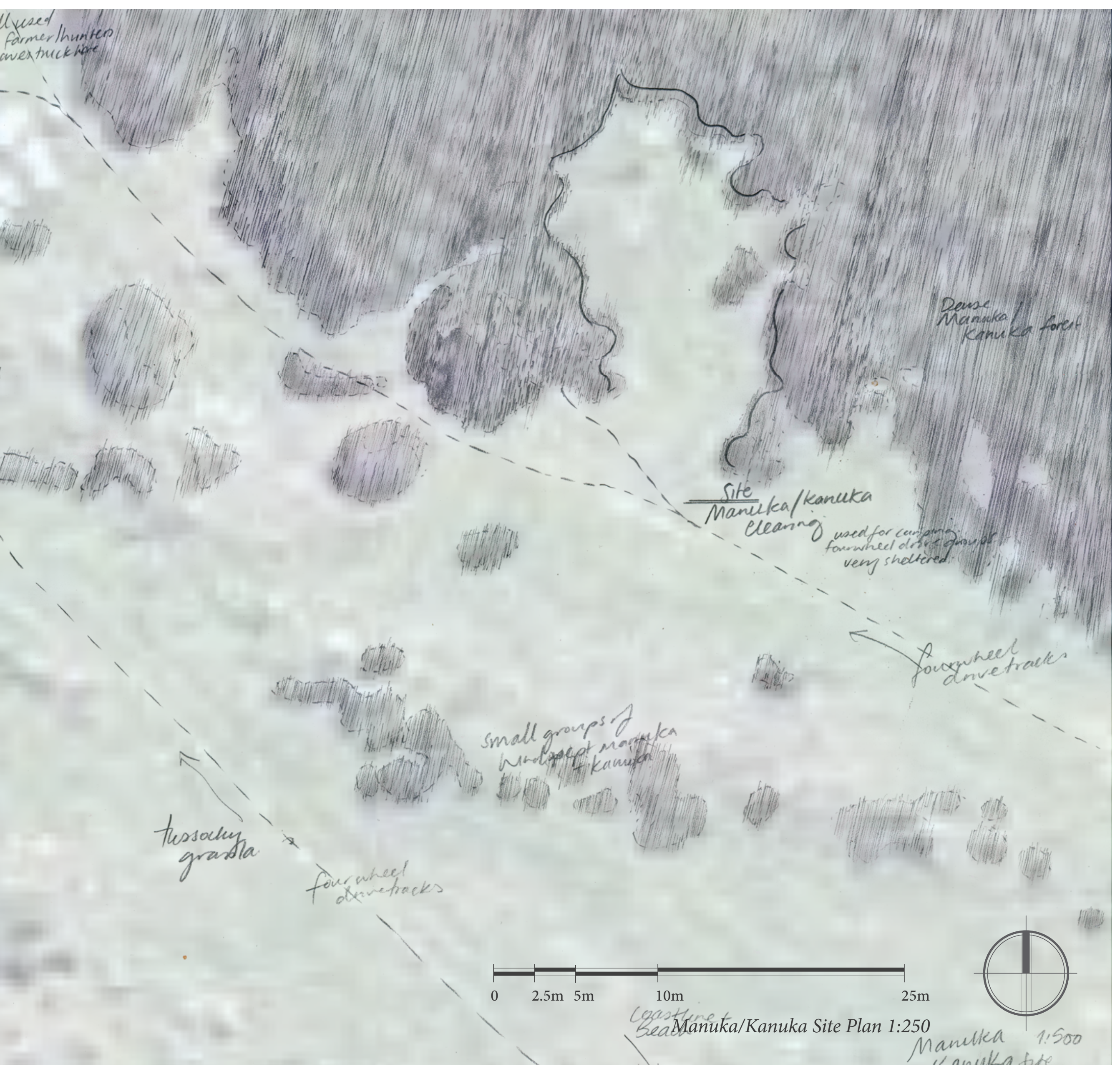



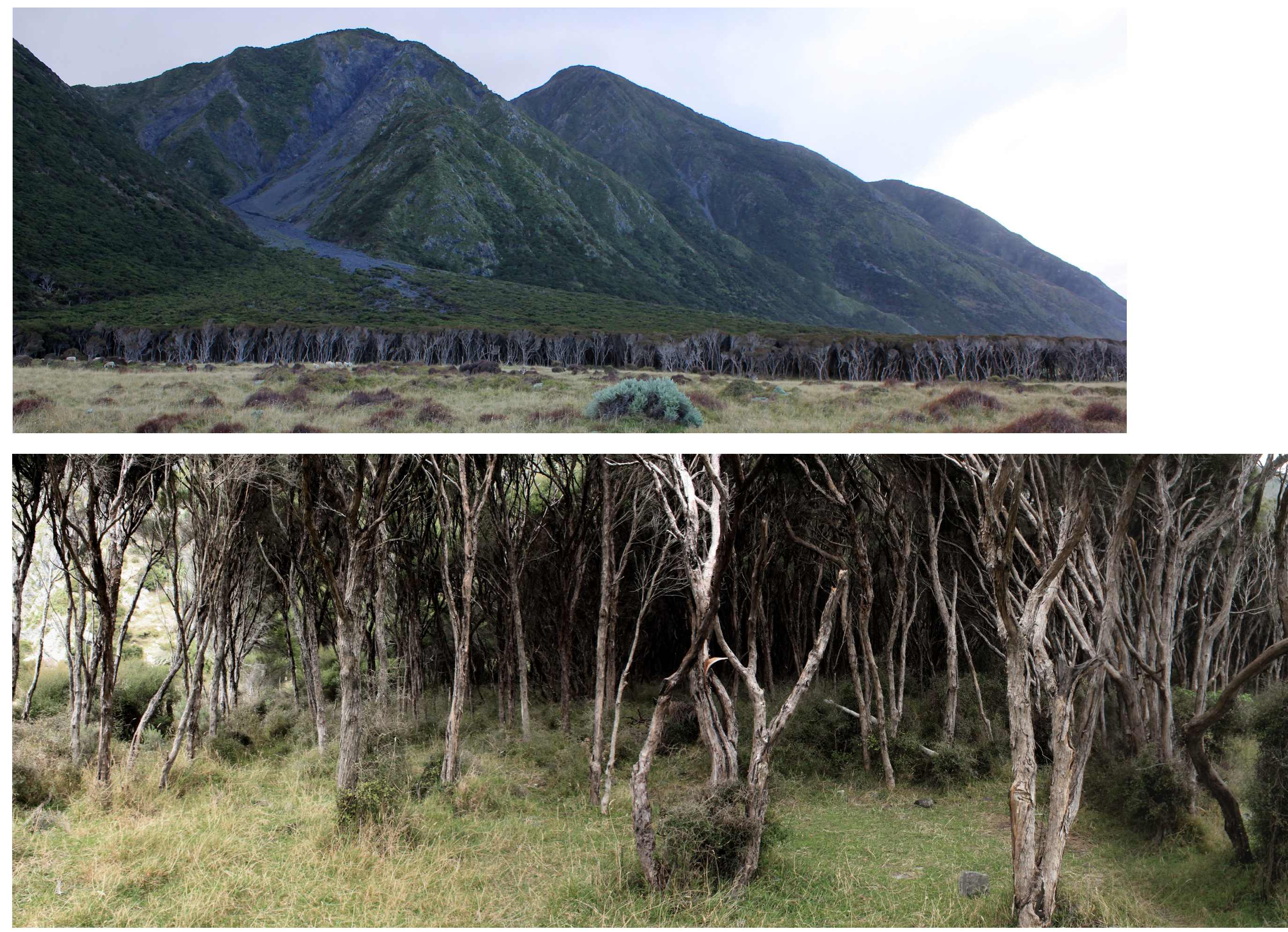

Figure 1.13

Manuka/Kanuka scree site photographs. 

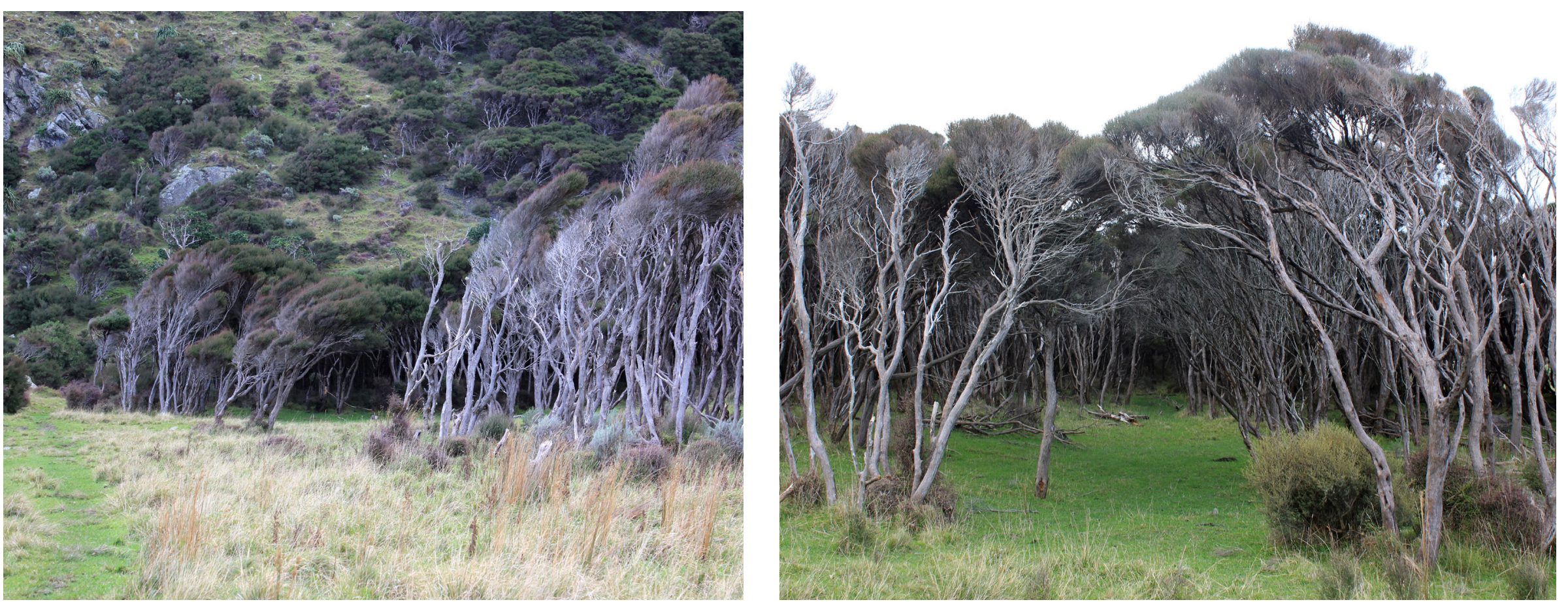

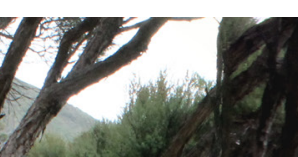

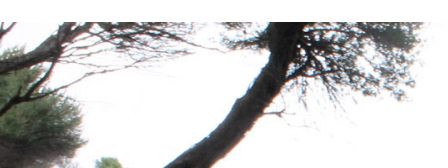

1. $+21,16$

in $-3 x^{2} \div$

$f(x)=3 x^{2}=$ $4 \times 46,3$ s. . 2 (2)

(ive) 1iv) (s) H.

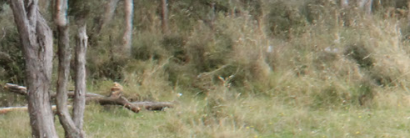

\section{1 texis}


The fourth site visit was undertaken with the intention of finding a specific site, taking measurements, further observations of the temporal nature of this coast and to explore the scales of permanence present within the chosen site.

\section{Boulder Site}

On the $13^{\text {th }}$ July I approached Ngā Pōtiki Reserve with this in mind and slowly made my way across the landscape navigating rocks and exploring. I found on closer inspection of the forested area that this was a dark, compressed and unappealing place to inhabit that did not embody the sense of temporality of site but resisted it. The very nature of the close-knit growth of the trees, the scrub-land beneath and the windswept canopy is designed to enable this ecological system to withstand the extreme conditions.

Instead the area that embodied the most movement was the exposed point scattered with rock debris from one of the earthquakes (refer to Figure 1.14). In particular, two large boulders drew my attention. Grooves are worn into the sides of the rocks in the passage between through years of gradual erosion from wind, water and sea spray. The rocks are a part of the surrounding environment but are removed due to their mass and the scale of permanence and slow change. Despite this, the sense of movement is noticeable through the contrast to all other elements such as plants and wind as they are forced to interact with the permanent insertions.

\footnotetext{
Figure 1.14 (right)

Boulder site plan, 1:250
} 


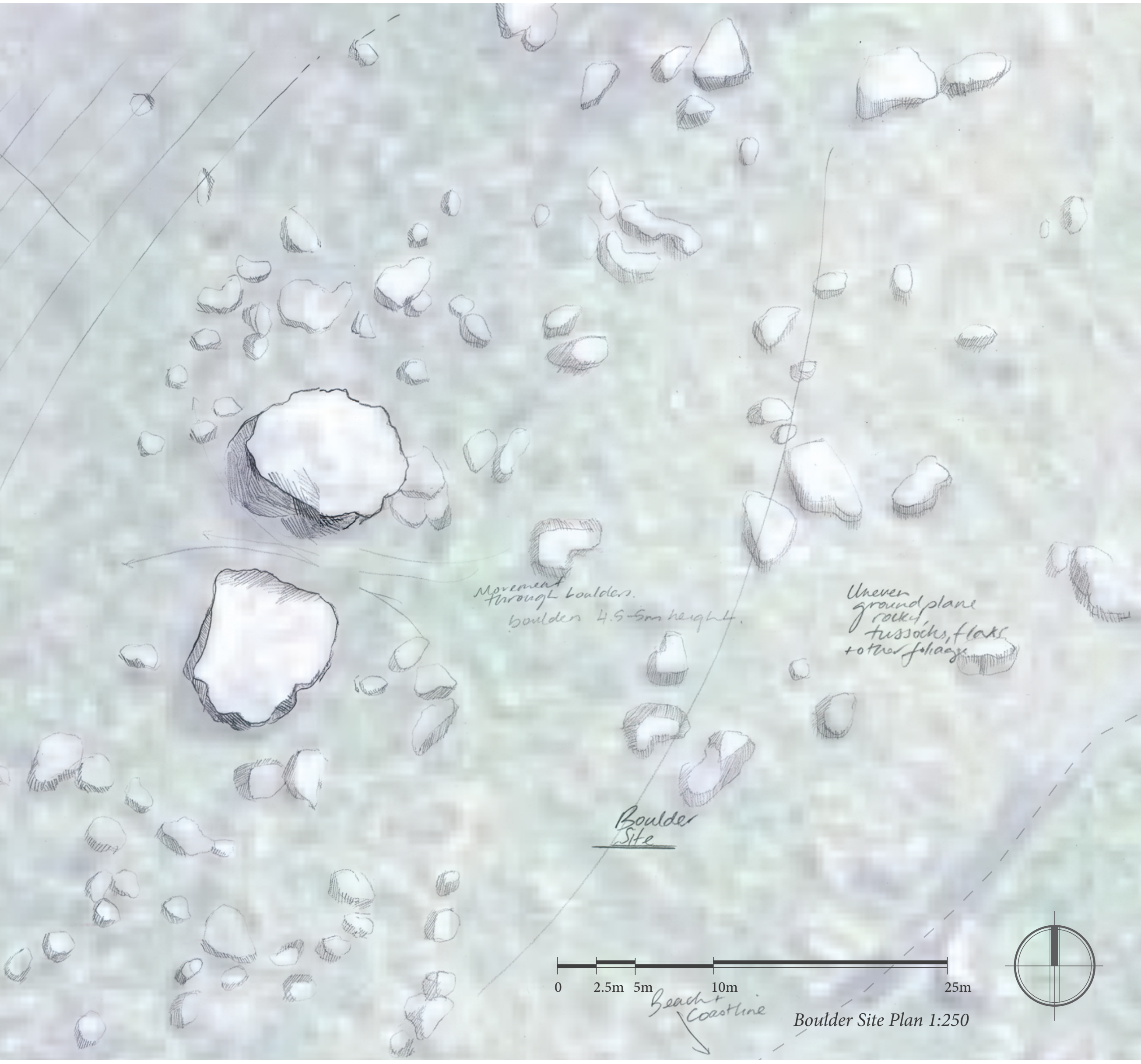




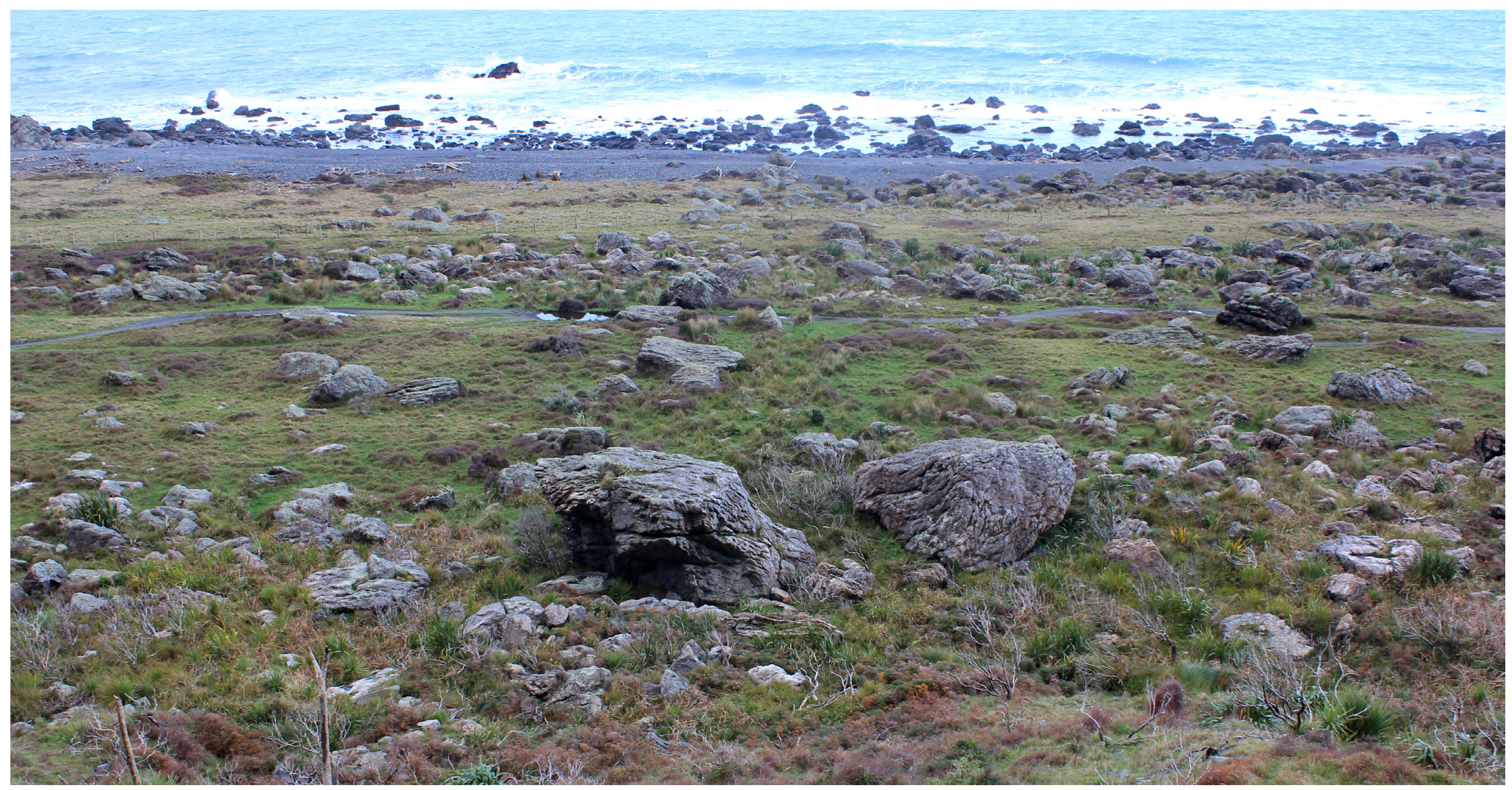

Figure 1.15

Boulder site aerial perspective from the steep hills above. 

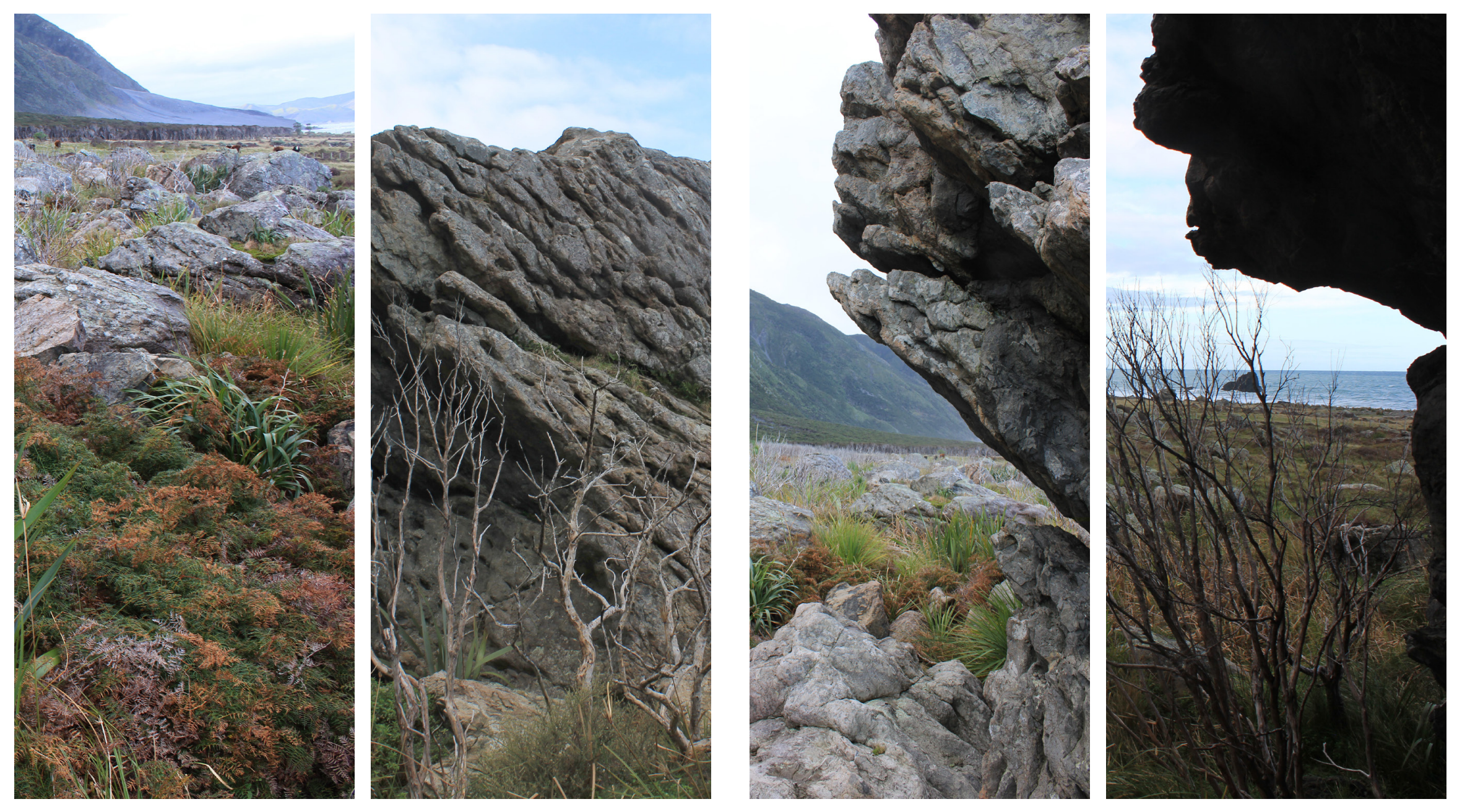

Figure 1.16

Boulder Site photographs showing ground terrain, movement lines worn into the boulders, the rough limestone, and the size of the rocks as I stand under them. 


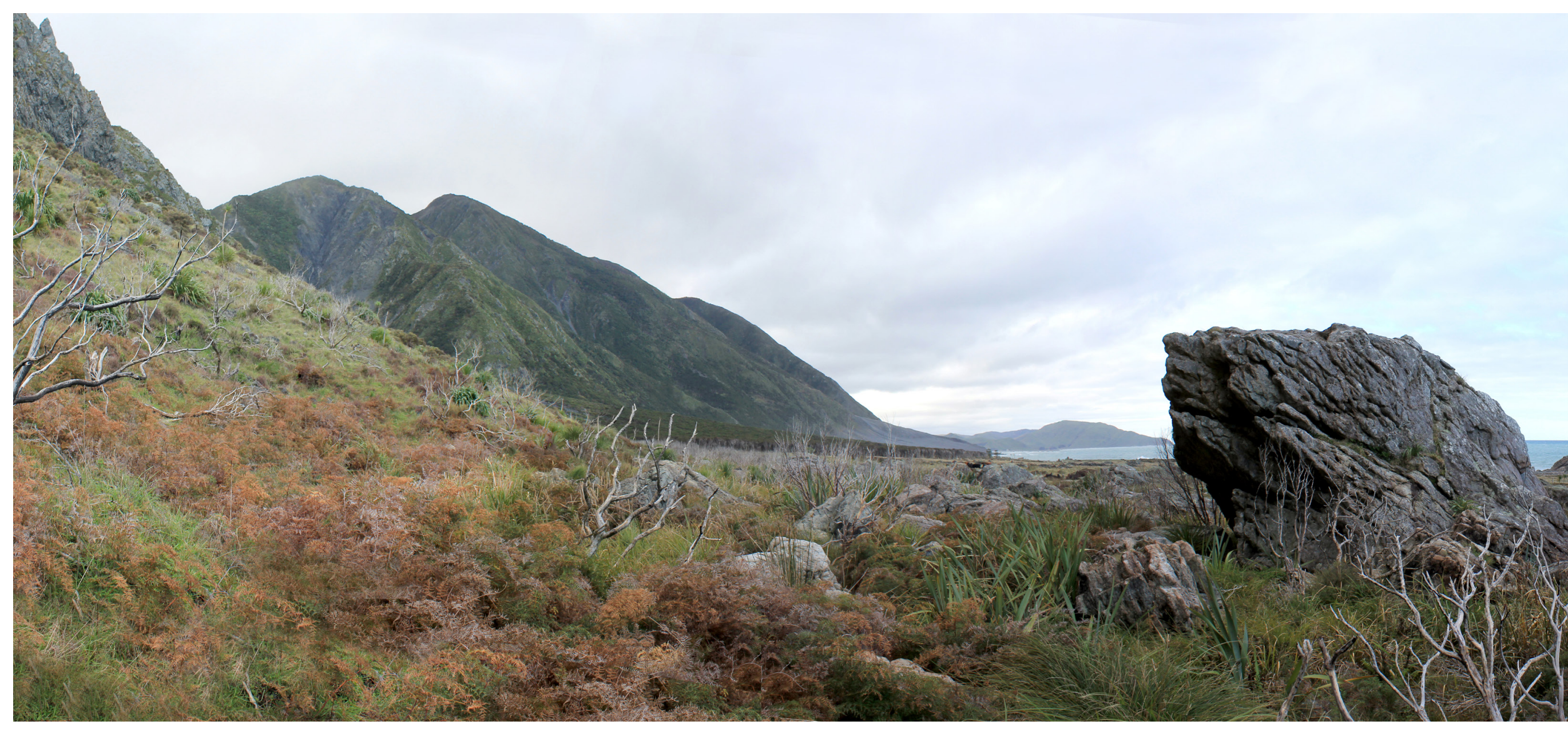

Figure 1.17

Boulder site panorama looking NE showing both boulders and the relation

to the terrain, ocean and mountains. 


\section{References}

1. B. Foss Leach, "The Prehistory of the Southern Wairarapa," Journal of the Royal Society of New Zealand 11, no. 1 (1981): 11.

2. A. P. Bruce, Indigenous Vascular Plants in the Vicinity of Cape Palliser, Southern Wairarapa, S.L.-1000Ft. (Ngawhi to Ngapotiki - N 168/9) (Wairarapa: Department of Scientific and Industrial Research, Botany Division, 1987), 1.

3. Due to the proximity to the ocean, high salt spray, potential in constant in ground contact and exposure to rain; external timber in ground requires a hazard class of $\mathrm{H} 4$ for durability. Although in the New Zealand Building Code it states that H5 is a requirement for house piles, as this is a semipermanent shelter the duration of external exposure is less, therefore $\mathrm{H} 4$ is acceptable.

New Zealand Wood, "Timber Performance and Durability," NZ Wood, accessed November 12, 2013, http://www.nzwood.co.nz/learning-centre/lctimber-performance-durability/.

4. "Black Beech," NZ Wood, accessed November 12, 2013, http://www.nzwood. co.nz/learning-centre/black-beech/.

5. "Rewarewa," NZ Wood, accessed November 12, 2013, http://www.nzwood. co.nz/learning-centre/rewarewa/.

6. “Totara," NZ Wood, accessed November 12, 2013, http://www.nzwood. co.nz/learning-centre/totara/.

7. "Hinau," NZ Wood, accessed November 12, 2013, http://www.nzwood. co.nz/learning-centre/hinau/.

8. Leach, "The Prehistory of the Southern Wairarapa," 25.

9. Ibid., 21 .

10. Graham Harris, "Rengarenga Lilies And Maori Occupation At Matakitakia-Kupe (Cape Palliser): An Ethnobotanical Study, By Graham F. Harris And Haami Te Whaiti, P 271-286," Journal of the Polynesian Society 105, no. 3 (1996): 271-286.

11. Graham Harris, "Maori Land Restoration: The Matakitaki-a-Kupe Project." 46-47, accessed April 27, 2013, http://www.rnzih.org.nz/pages/NZ-Plantsand-their-Story-45-50.pdf.

12. Boffa Miskell, Wairarapa Landscape Study 2010; Landscape Character Description Report (Greater Wellington Regional Council, August 2010), 80.

13. Leach, "The Prehistory of the Southern Wairarapa," 11.

14. Ibid.

15. Bill Dolson, "Landscape Temporal Variations," Bill Dolson, February 24, 2007, http://www.billdolson.com/Variations/variations.htm. 
34 


\section{CONTEXT}

This section positions the research within theoretical, art and architecture contexts defined through literature and case studies. The three key areas of wilderness, affect and non-static will be discussed as well as a small section on glamour and a selection of key precedents will be introduced.

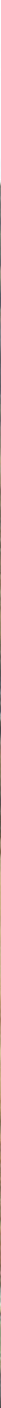




\section{Wilderness}

A place for people, a place for design?

The view of wilderness has changed remarkably throughout history. Two main perspectives have emerged in literature; the first of 'pure naturalness' and the second of a cultured nature. These perspectives are not reflected in the studies of how people interact and inhabit nature. In response to this misalignment, this thesis proposition instead emphasises a new middle ground.

The first approach has arisen from the environmentalist movement. The 'nature knows best' view supports the idea that nature is a pure and pristine environment and this should remain separate from the social culture of human activity. ${ }^{1}$ The term 'visitor' in the landscape is evidence of this viewpoint and dictates many levels of New Zealand wilderness experience, especially with Department of Conservation and other conservation bodies. ${ }^{2}$ There is much critique of a pure naturalness as it considers the health of the landscape and maintaining ecosystems in a retrospective gaze. ${ }^{3}$ The human inhabitation of these landscapes is seen as degrading and this approach refuses to acknowledge the embedded cultural history of many wilderness areas.

The second perspective is the idea of 'cultured naturalness' a coexistent approach to human inhabitation of natural landscapes, as wilderness itself is a cultural construct. ${ }^{4}$ Abbott draws upon the perspectives of authors such as Park (2006), Turner (1996), Cronon (1995) and more; to emphasise the need for the person to be an occupant of the landscape to which they belong. This view is based on a complementary relationship between culture and nature where the natural environment is not compromised but enhanced by the presence of humans. Braun and Castree, and Newton et al., reinforce that the crucial issue is taking responsibility for how we express our relationship with nature, with what consequences and to whose benefit. ${ }^{5}$
Theoretically, authors such as those mentioned above reinforce an equal balance between culture and nature, but as the following studies show, the general public understanding of this view is weighted toward cultural gain. In 1998 and 2000 Newton, Fairweather and Swaffield conducted two surveys to determine if these two approaches were present in the views of the New Zealand public. They discovered that "cultured naturalness perspective does not necessarily reject the concept of wild nature, but thinks about the physical environment primarily in terms of human relations and activities". Careful consideration of the interventions in nature is compromised by economic or social gain, and the environment begins to be treated as a commodity for human consumption and/or leisure practices. ${ }^{7}$

This misalignment between theoretical discussion and public understanding reinforces the need for a practical (as opposed to terminological) method of reintroducing a balanced relationship between nature and culture. This thesis builds on Abbott's investigation of the potential for landscape architecture to generate a dialogue with the public to alter the perception of nature. ${ }^{8}$ Architecture and sculpture as design disciplines also have potential for this dialogue. The shelter design proposes scaled levels of inhabitation of the conservation estate to satisfy both pure naturalist and cultured naturalness views.

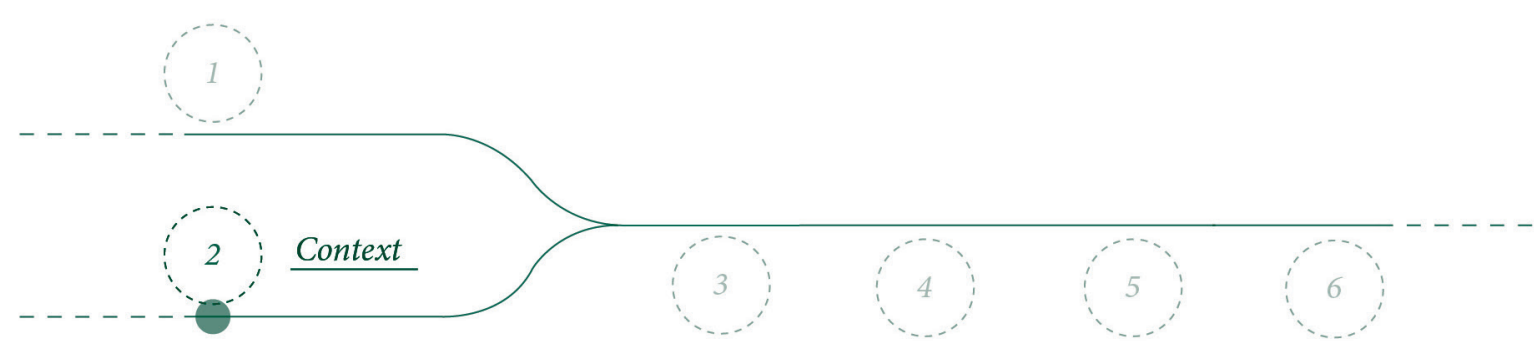


Central to this theoretical position and design investigation is the use of the term wilderness. Abbott rejects wilderness terminology due to the associations with an unspoilt, untouched remoteness; and instead replaces it with the term landscape, as he believes it can "open up deeper cultural potential in the conservation estate." ${ }^{9}$ In fact; it is the dialectical relationship between culture and 'wilderness', and therefore wilderness and architecture that establishes a rich context for my research. By challenging the most natural and isolated understanding of nature, through juxtaposition with design, this architecture has the potential to shift the public perception and heighten people's awareness of the need for a balanced relationship between nature and culture.

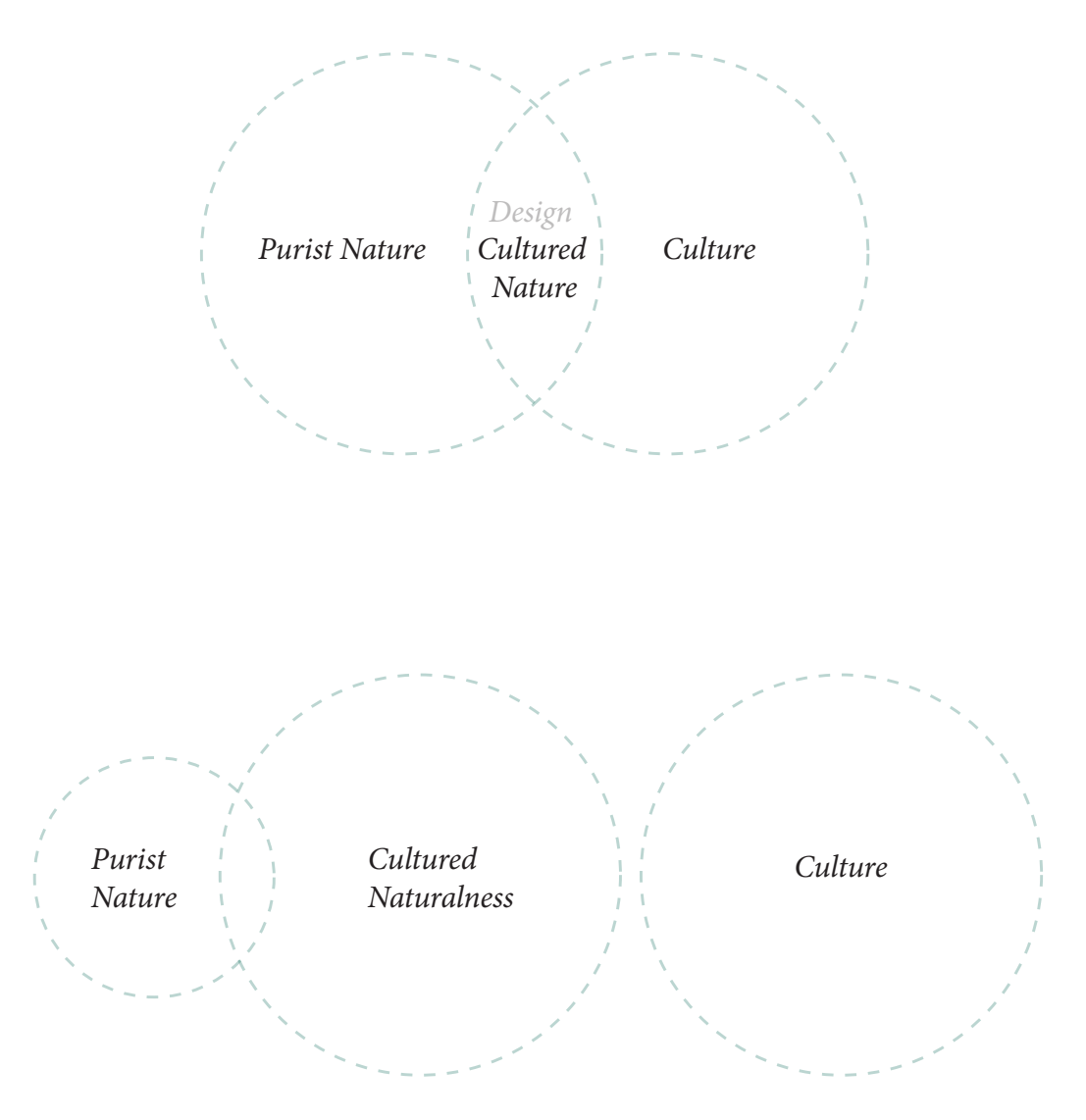

Figure 2.1 (top)

Theoretical perspectives of the wilderness - culture relationship

Figure 2.2 (bottom)

Public understanding of wilderness - culture relationship 
Affect

\section{What is Affect?}

Affect is a preconscious reaction that forms the framework for understanding of how a person connects with their surroundings. This is explained most simply, through the relationship to feelings and emotions. Four different translations of affect will be discussed followed by closer investigation into the 'Affect Drive Theory' approach, and the 'Deleuzian/ Spinozian' approach. These two approaches provide a framework of understanding that informs the active designing.

Eric Shouse clearly defines the relationship between affect, feelings and emotions..$^{10}$ Affect is the first preconscious reaction between 'bodies'. A body here refers to unlimited substance; thoughts, ideas, objects, people and things. In many approaches, affect is the push that inspires people to act. ${ }^{11}$ Feelings are a sensation that has been checked against previous memories and identified, for example, anger, happiness, disgust, sadness etc. ${ }^{12}$ Whereas emotions are the projection of a feeling upon the face and/ or through body movements. ${ }^{13}$ It is important to note here that both feelings and emotions are influenced through social conditioning therefore, unlike affect, are conscious and cognitive. Therefore, affect is the level of experience that removes social and cultural conditioning to reveal an embedded resonance.

Each of the following four translations of affect relate to this basic idea of affect as push or intensity but with subtle differences in the results (refer to Figure 2.3). Nigel Thrift outlines each of these four areas as 'Embodied knowledge', "Neo-Darwinian' theory, Affect theory' and 'Spinozian/ Deleuzian theory. ${ }^{14}$

The first approach considers affect as embodied practices that are displayed as emotions. This embodied translation of affect is based in the phenomenological tradition and is concerned with what drives emotion. Sensory perception is intertwined with response in a constant sensing and visual display of bodily states that is focussed heavily on human interaction. ${ }^{15}$
The second translation, Neo-Darwinian theory, is a naturalistic approach grounded in evolutionary understandings of people and animals alike. This is again focussed around affect as the potential for action, which is then displayed involuntarily upon the face and through the body of the animal. This perspective establishes the relationship between affect and emotion as inseparable and even raises evidence about five universal emotions and expressions such as anger, disgust, enjoyment and fear. ${ }^{16}$

The third and fourth approaches are described by Thrift as separate translations. Some ideas within each theory are opposing but in my opinion in combination, they have can be applied to the architectural field.

The third approach originated from Freudian's model of affect as a physiological 'drive' related to desire and sexuality that forms the "root source of human motivation and identity". ${ }^{17}$ Other authors such as Tomkins (Demos, 1995) ${ }^{18}$ and Sedgwick and Frank (1995) ${ }^{19}$ critique the simplicity of this theory and expand by distinguishing between survival drives such as; eating, breathing, drinking, sleeping and excreting, and 'other' drives. Tomkins states that survival drives are time-limited, concentrated on particular aims, eating, sleeping etc, and concentrated on specific objects. ${ }^{20}$ Whereas, other affects that are connected with emotion such as enjoyment or anger, are without a defined aim, a defined object and with no time limit; for example a moment of anger could inspire a feeling of sadness that could last for days. Importantly the emotional affects can be attached to anything, "people, ideas, sensations, relations, activities, and any other number of other things, including other affects ${ }^{21}$ 
The last Spinozian/Deleuzian approach explores the notion of emergence and how an interaction between single and multiple simple bodies can create a complex reaction in a person, affect. Here affect is a 'reaction' to an interactive encounter that takes the form of an increase or decrease in the ability of the body and mind to act simultaneously. ${ }^{22}$

The scope of this thesis will be limited to these two final translations of affect as they deepen understanding of a person's interaction with space, (refer to Figure 2.3, pg 44).

The distinction between time-based drives and affects that translate into emotions is of interest as it begins to explain the differences in how people inhabit architecture. Time-based drives are essential needs, catered for by programmatic functions such as cooking facilities, cleaning and sleeping areas. This base necessity is viewed as a foundation, the minimum requirement of space. Drives that are not confined by time, object or aims, add a richness to experience that forms a physiological connection of mind and body; a connection that can heighten awareness of both the natural environment and the architecture.

The Spinozian/Deleuzian approach builds from this base of drives and through the understanding of affect as a reaction creates a potential for increasing affect through design. Nigel Thrift in his analysis of the engineering of affect in modern society defines theories relating to Spinozian and Deleuzian theories that can be applied to architecture to increase affect. First, the model of tending;

"Here the simple political imperative is to widen the potential number of interactions a living thing can enter into, to widen the margin of 'play', and, like all living things, but to a greater degree, increasing the number of transformations of the effects of one sensory mode into another." ${ }^{23}$
The second theory relates to affective drives by increasing affect through the manipulation of space and time. Thrift refers to slow motion film and other arts that either speed or slow time to allow new resonances, a heightened level of detail and a different experience to what would be registered in real time. ${ }^{24}$

Both models; of tending, and the manipulation of space and time, are applied theories based within frameworks of understanding affect. This thesis investigates if these theories can be translated into architectural moves where the building acts as a mechanism to heighten the resonance a person has with a place. 


\section{Non-Static}

\section{The Interactive Architecture Paradigm}

"The desire to harness and maximise the affective qualities of an object - to animate the seemingly static - has a long and diverse lineage in the history of architecture". 25

In this design research investigation, non-static architecture acts as the mechanism to highlight the changing interaction between people, wilderness conditions and the built form. This literature review investigates present trends within the field of non-static interactive architecture including analysis of relevant mechanical, biological and sculptural case studies to define the balance between functional and experiential for this design research. The concept of movement will be discussed as central to this approach as it ties together the temporality present in the natural landscape and theories on increasing affect.

The argument for interactivity is formed from a resistance to buildings that are conceived as enduring and designed to be built for eternity. ${ }^{26}$ As Fox and Kemp explain, the motivation behind this architectural trend is to meet the constantly changing individual, social, and environmental demands. ${ }^{27}$ Currently this trend is evolving through emerging technologies such as ubiquitous computing, robotics and nano-composite materials. Developments that are more recent signal a shift from a mechanical paradigm of efficient environments to a biological paradigm. ${ }^{28}$ The shift criticises the mechanical as more focused on functional, performancebased technological spaces and reinforces that the biological paradigm requires awareness of "aesthetic, conceptual and philosophical issues relating to humans and the global environment". ${ }^{29}$ This trend reflects the growing recognition of the wealth of knowledge potential embedded in our surrounding natural environments, as well as a developing international focus on renewable resources, conservation and sustainability
Both of these trends are explored through programmatically functioning environments but Fox and Kemp reinforce the potential for a heightened user experience in interactive artistic as opposed to interactive architectural initiatives. They emphasise a number of qualities that are applicable to this design brief for a glamping shelter, such as; no permanency constraints, a sense of playfulness, lower cost design, transportability and the possibility for greater public exposure..$^{30}$ They go on to reinforce the link between an artistic initiative and a heightened connection between users and their environment:

"This lack of permanence not only allows for interactive systems to exist in nonstandard locations, but it also allows for looseness in user experience, whereby users may be involved who would not ordinarily participate in an architectural setting". ${ }^{31}$

Non-static interactive architecture, in the context of this thesis is defined as an interactive system that dynamically occupies an undefined space to generate a conversation between multiple bodies; people, needs, ideas, land, weather conditions and more.

The two theories to increase affect outlined by Nigel Thrift of tending, and the manipulation of space and time, ${ }^{32}$ can both be achieved through movement and change. Movement is not the only way to translate these theories into a built form but is the most interactive to link with Thrift's notion of tending and reflects the buildings temporal setting. When a building is static, it can be clearly defined in the mind of the occupant so preconceptions are always accurate. Bhatt, Dylla and Hois explore the notion of an undeterminable and deformable space that can become indefinable in the mind of the occupant. ${ }^{33}$ These unknown's create the possibility for repetitive moments of surprise and connection with each encounter. Spinoza reinforces this perspective by stating that affect is generated through the simultaneous thinking and doing, so that "knowledge 
tending, the constant change widens the number of different interactions the occupant has with the space and therefore has a potential to increase affect.

The second more practical theory of the manipulation of space and time can be achieved through non-static architectural detailing. This will be elaborated on later in the design phase but is based around the idea that the architecture can embody movement to visually interact with wind, a changing, uncontrollable and invisible condition. The design of the building and especially detailing can take this wind and distort the result, for example a fluid system with tight jointing will require a lot of force to move and create a slow motion effect. 


\section{Affect Theories}

\section{Embodied Knowledge}

Strong link to emotion

\section{Drives}

Affect as potential for action

$$
\text { Strong link to }
$$

human interaction

Phenomenological

Focussed on emotional drives

Constant sensing and displaying

Human interaction
Naturalistic

Evolutionary

Affect - potential for action

Affect and emotion inseperable

Five universal emotions

\section{Scope - Architecture}

\section{Figure 2.3}

Visual diagram comparing the different translations of affect and displaying the theories explored in this thesis and their relationship to architecture.
Neo-Darwinian

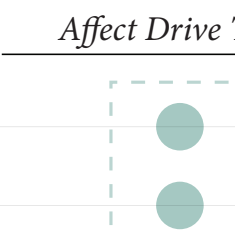

Spinozian / Deleuzian

Physiological 'drives'

Root source of human motivation and identity

Drives related to sexuality and desire

Critiqued; Tomkins (1995)

Sedgwick and Frank (1995)

Diffrentiates between

time-limited survival drives

and 'other' drives with no time

constraints

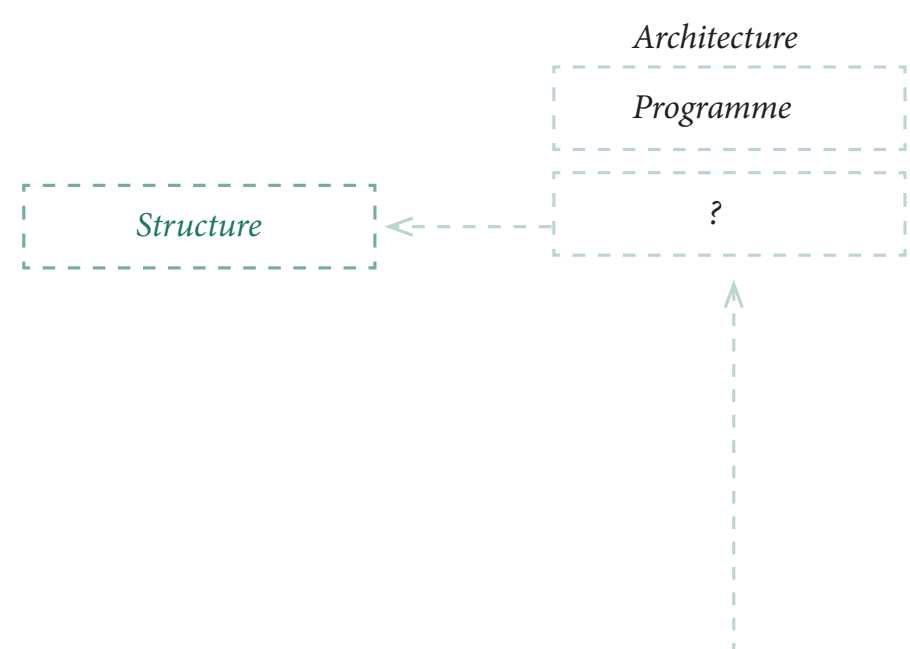

Temporality

Movement
This theory provides the framework for understanding how people inhabit space.

Survival Drives - Sleeping, eating, drinking etc. 'Other' Drives - Experience, emotion and 'Other' Drives - Experience,
as Affect connection.

\section{Spinozian / Deleuzian}

Nigel Thrift introduces two theories to increase affect, experience, emotion and connection.

1. Tending - increase potential number of interactions

2. Manipulation of space and time - the use of a mechanism to create a different experience 


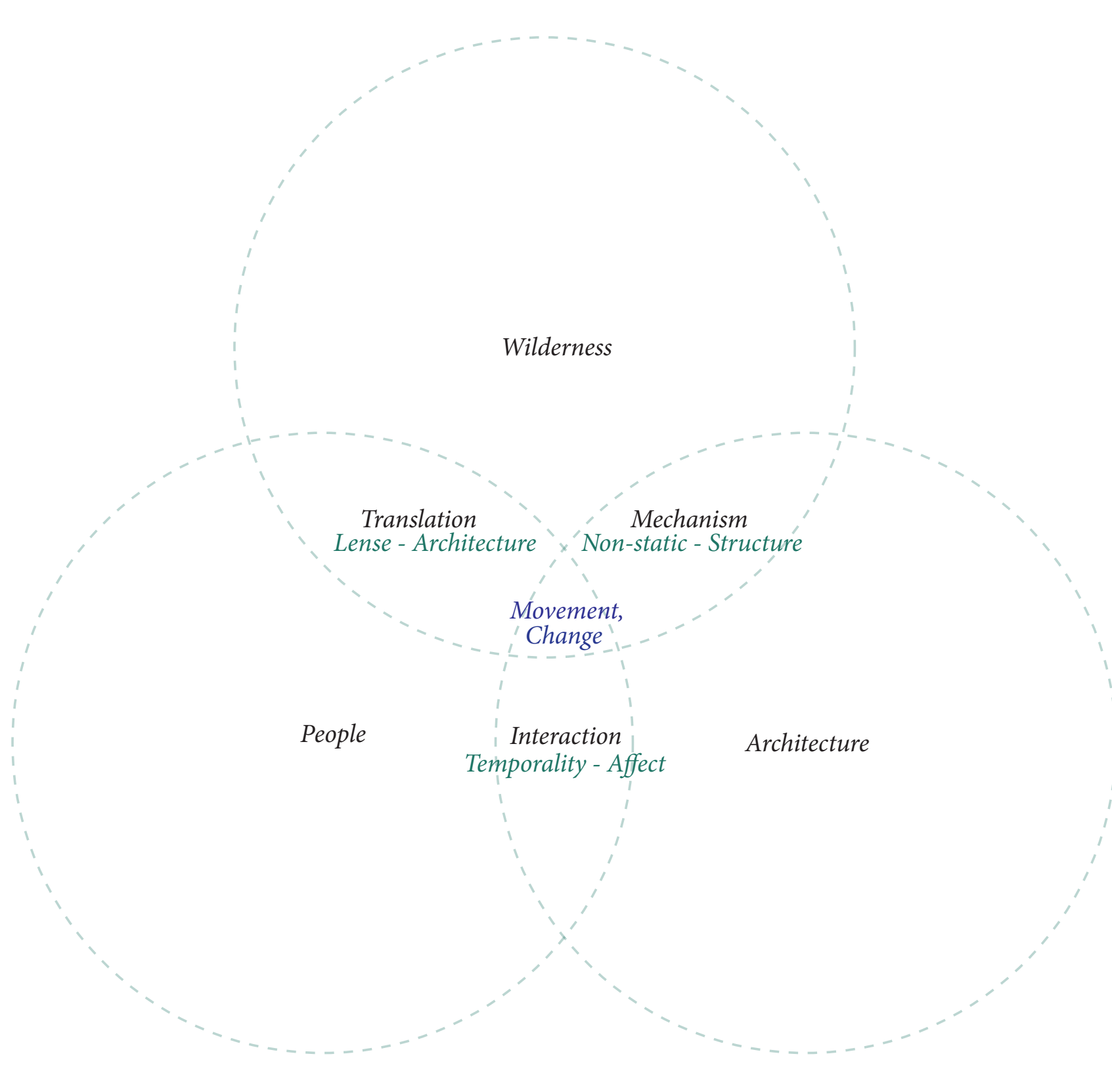

Figure 2.4

This diagram expresses the intention of this research to heighten people's awareness of wilderness and architecture.
The cross-over sections describe how this will be achieved. The key ideas are summarised in the centre. 


\section{Glamping}

The notion of a glamorous camping shelter has evolved from the African safari tours and other adventure tour based camping where an increasing demand from American and European tourists sought high quality accommodation in wilderness locations. ${ }^{36}$ Glamping is designed as an experience that removes all negative attributes of camping, such as; no space, leaking tents, uncomfortable bedding and compromised food, to be replaced with luxury versions. Glamping appeals to a wider demographic of people, drawing them into wilderness settings. Where a basic tent and self-sufficiency may appeal to the outdoor enthusiasts, urban dwellers require more motivation to venture into an isolated and somewhat extreme landscape environment.

"Camping is a form of outdoor recreation that is part activity and part accommodation"37

This glamping shelter design seeks connect people to the landscape for the following three reasons. Firstly, the motivation for people to inhabit natural landscapes for recreation such as camping, glamping or staying at a holiday Bach relates to Maslow's Hierarchy of Needs to fulfil desires related to lovel belonging, esteem and self-actualisation. ${ }^{38}$ The pull of an outdoor experience begins with the need to escape daily routine. ${ }^{39}$ According to Mannell and Iso-Ihola (1987), escape is the primary motivator followed by personal rewards related to "self-determination (one's ability to exercise freedom in choosing a leisure activity), sense of competence or mastery, challenge, learning, exploration, and relaxation. ${ }^{40}$
This sense of pull to inhabit natural spaces leads us to the second important motivator for increasing people's contact with natural environments. This is aligned with recent empirical research into the physical and psychological benefits of 'healing landscapes'. Green and blue landscapes such as oceans, lakes and forests are proven to be therapeutic to; lower stress, cure diseases such as burn-out and depression, have a positive effect on mood and ability to concentrate, and provide health benefits associated with encouraging physical activity. ${ }^{41}$ Brooker and Joppe link this research with public surveys in Britain that reveal that campers are aware and perceive the experience as increasing their general wellbeing. ${ }^{42}$ The design of a glamping shelter acts around this context in two ways; as a self-motivator to draw people into the wilderness, and to develop architecture that entices people into an environment that has so many positive effects on their being.

Thirdly, the term glamour has strong associations with a sector of society that is dominated by wealth and status. It is the field of art this is most applicable here as art and sculpture appeals to demographics that seek an unusual and rare experience. Fox and Kemp emphasise that sculptural fields are not often constrained by functional requirements of space, safety or other construction risks. ${ }^{43}$ The sculptural aim is solely to create an experience and impact often-large audiences. Architecture that is sculptural suggests a level of affective or experience focussed interaction that surpasses the pragmatic associations with camping or architecture. 


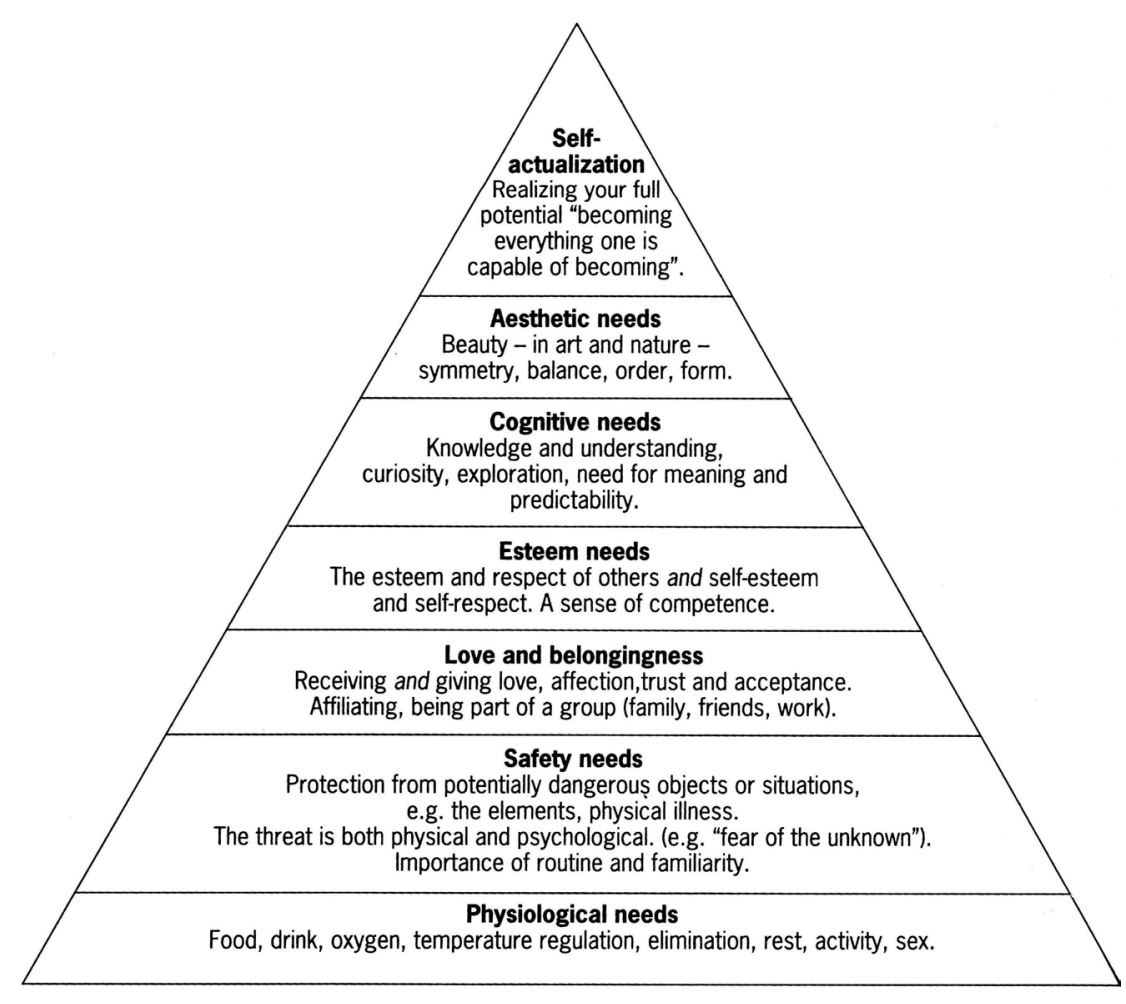

Figure 2.5

Maslow's Heirachy of Needs. ${ }^{35}$ 


\section{Precedents}

The following case studies look at the potential for architecture to act as the mediator to allow people to connect with the wilderness environment.

\section{Wilderness Case Study - Back Country Hut}

New Zealand Department of Conservation Backcountry Huts:

Waimakariri Falls Hut, Arthurs Pass National Park ${ }^{44}$

Mt Angelus Hut, Lake Rotorua, Nelson Lakes National Park ${ }^{45}$

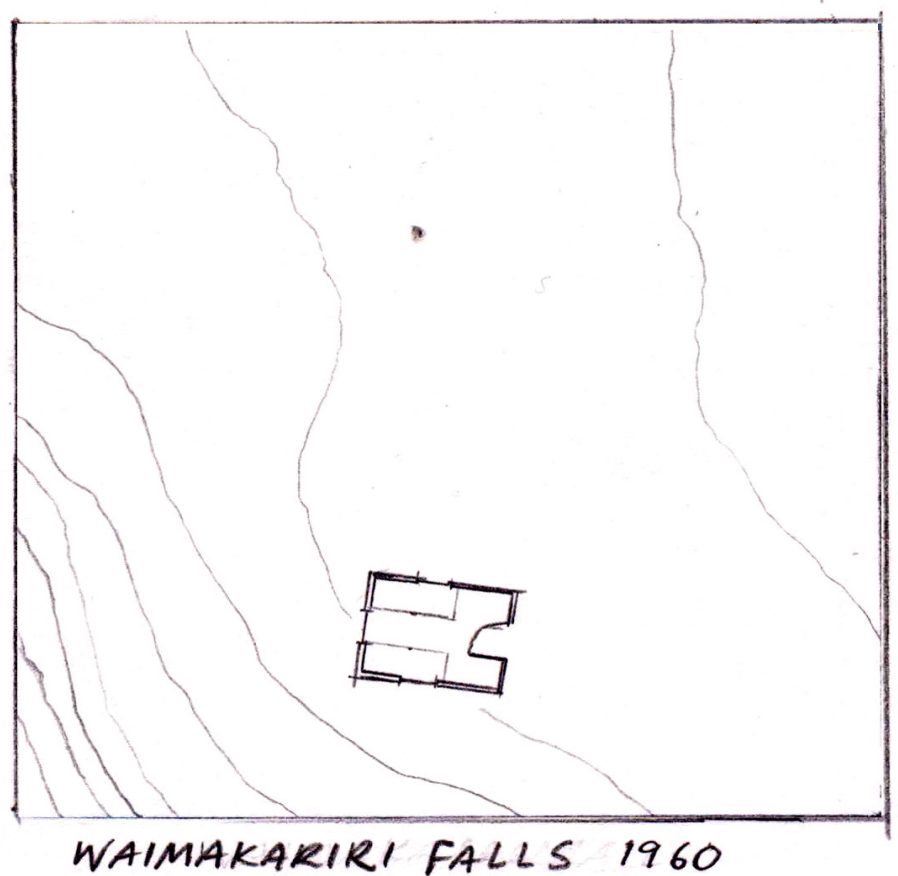

Figure 2.6

Backcountry huts precedent one, Waimakariri Falls Hut Plan, 1:20.
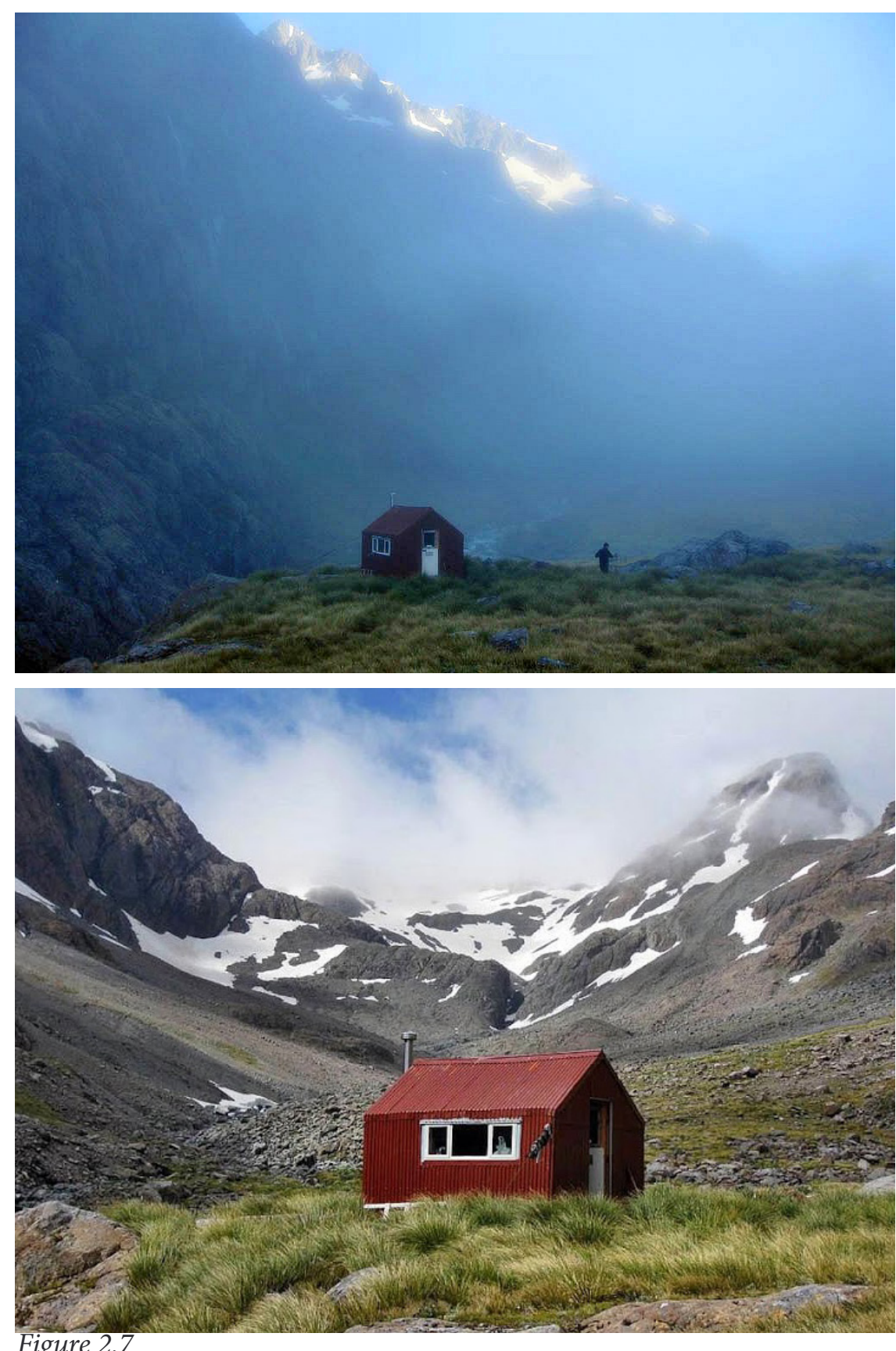

Figure 2.7

Backcountry huts precedent one, Waimakariri Falls Hut ${ }^{44}$ 


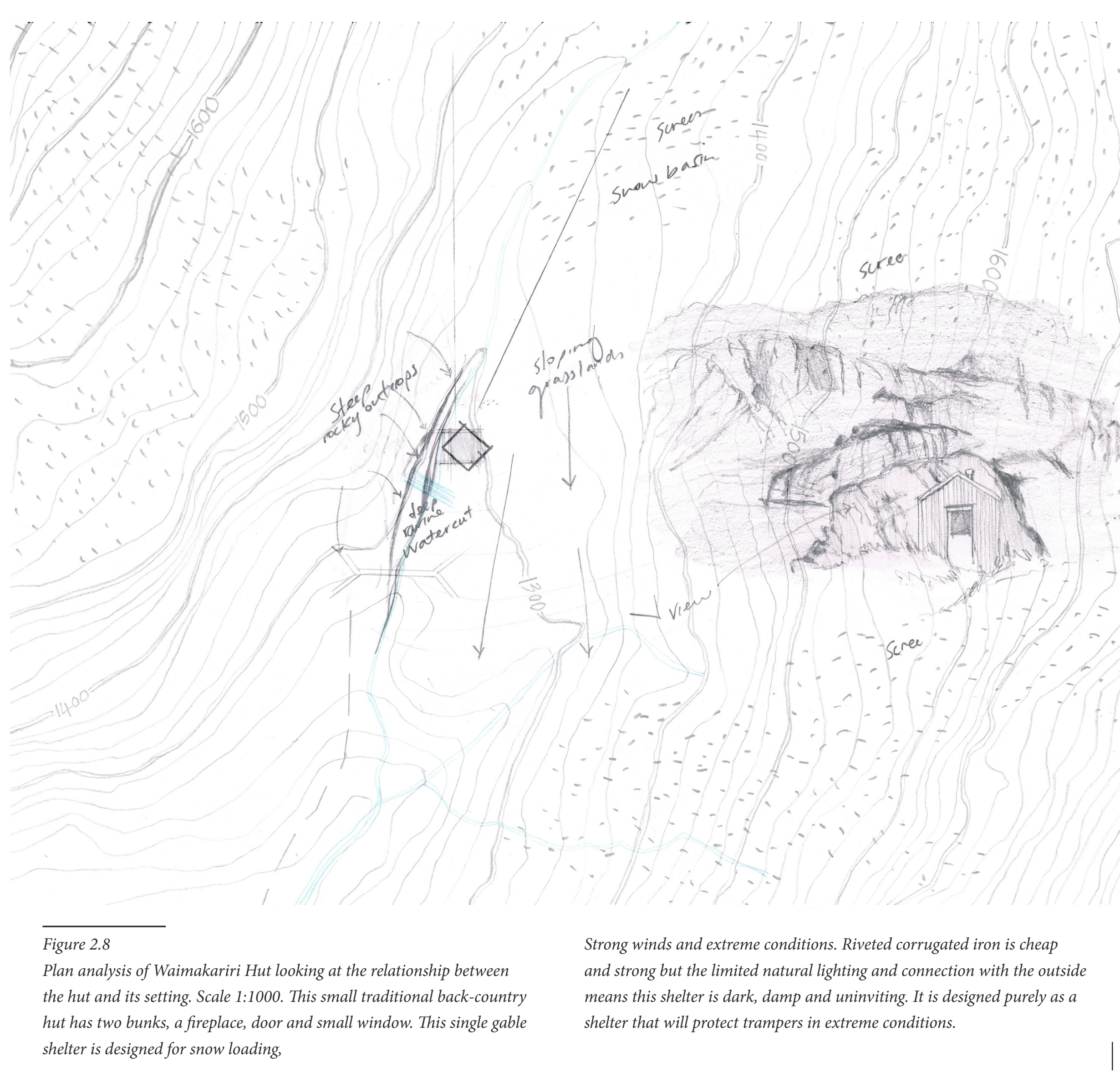



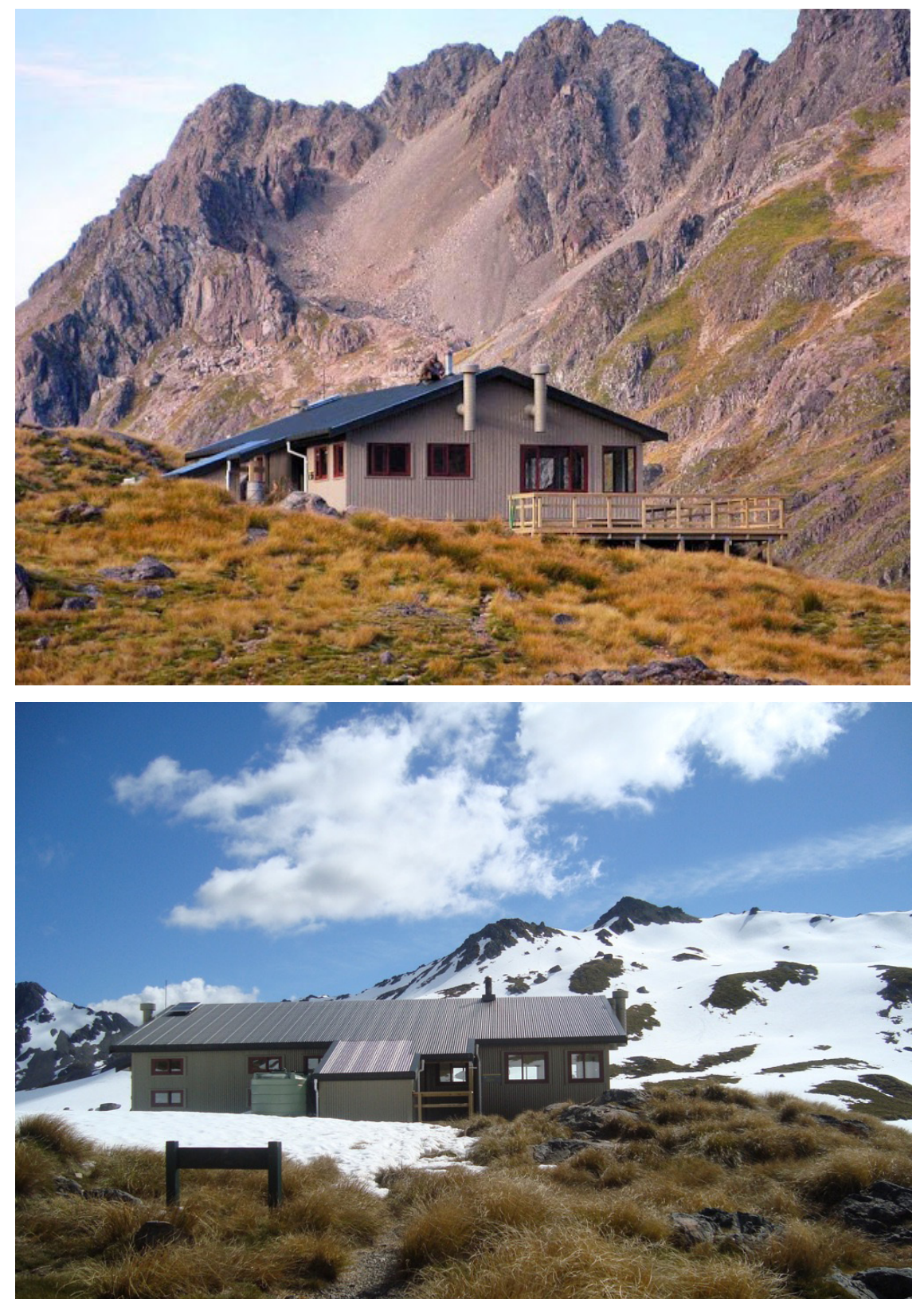

Figure 2.9

Backcountry hut precedent two. Mt Angelus Hut ${ }^{45}$

This new hut replaced an old smaller dilapidated hut with a new highly insulated 28 bunk hut.

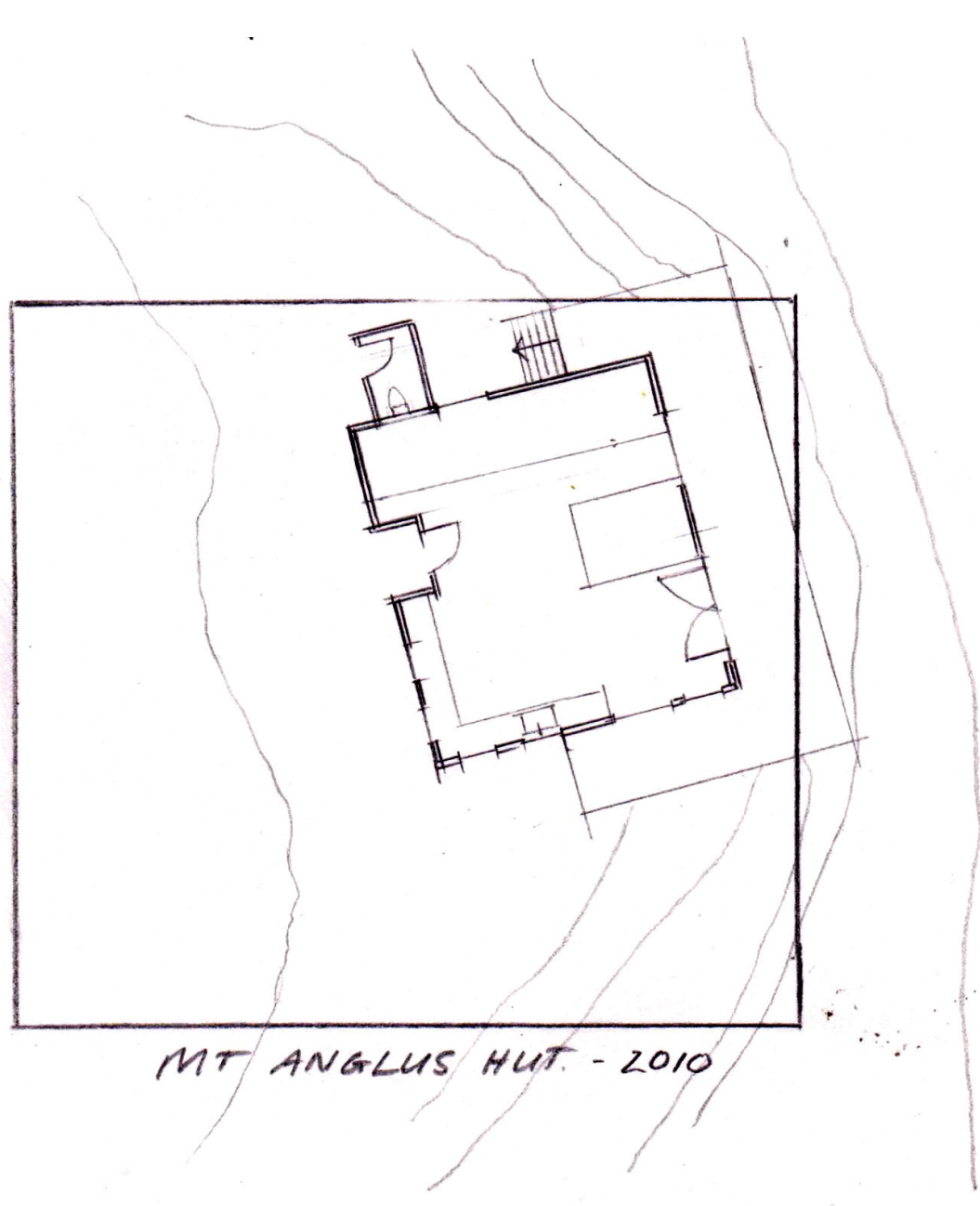

Figure 2.10

The new Mt Angelus Hut Plan, 1:20.

This hut has an increased interaction with the surroundings with a large external deck and windows. The single large internal space is well lit and warm with communal dining,cooking and sleeping areas. 


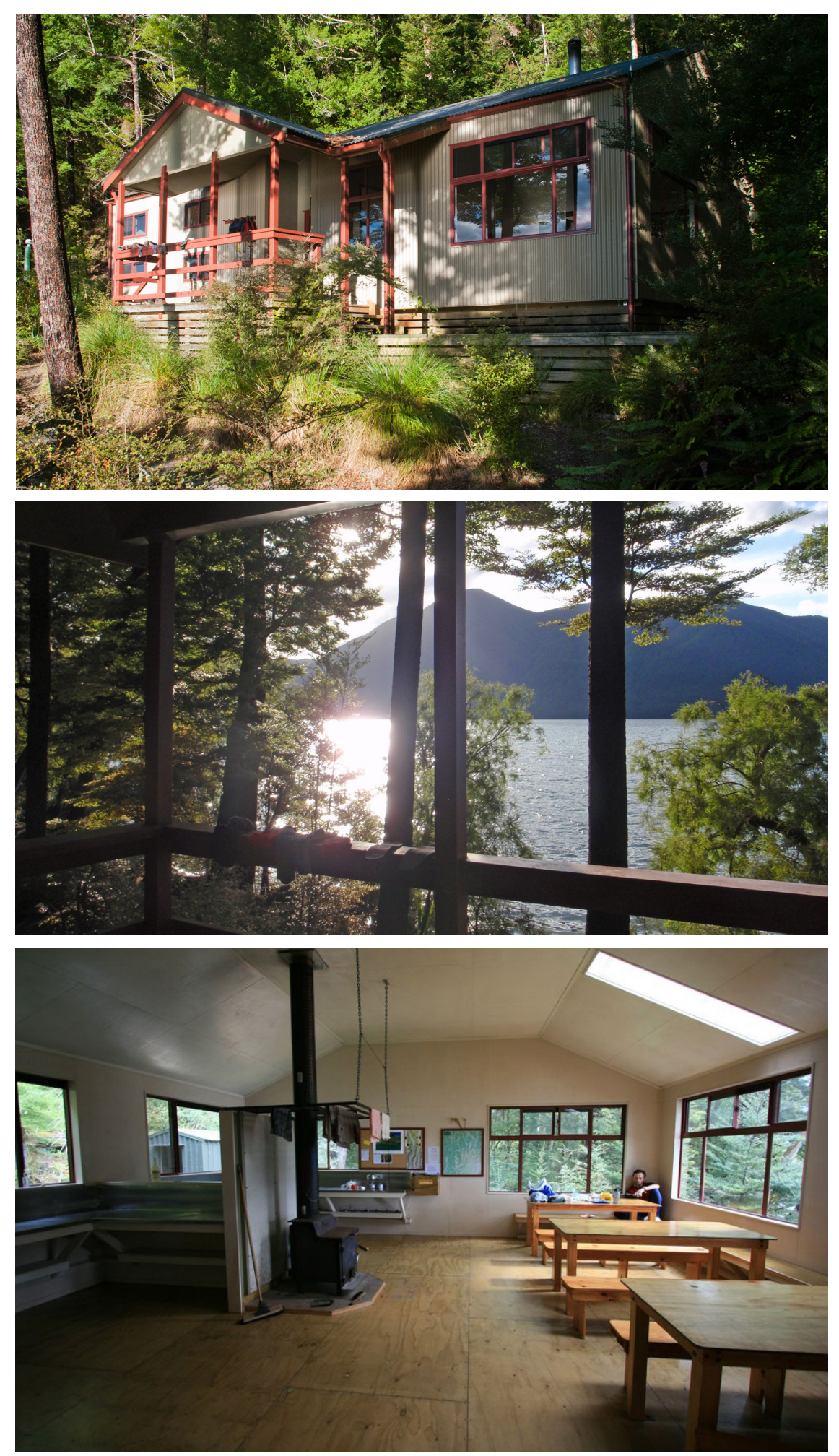

Figure 2.12

Backcountry hut precedent two, Sabine Hut. Photographs as follows; Sabine Hut exterior, view out to Lake Rotoroa and interior perspective of kitchen dining space." The interior shot can represent the majority of DOC huts as

their design standards are applied to nearly all backcountry huts.

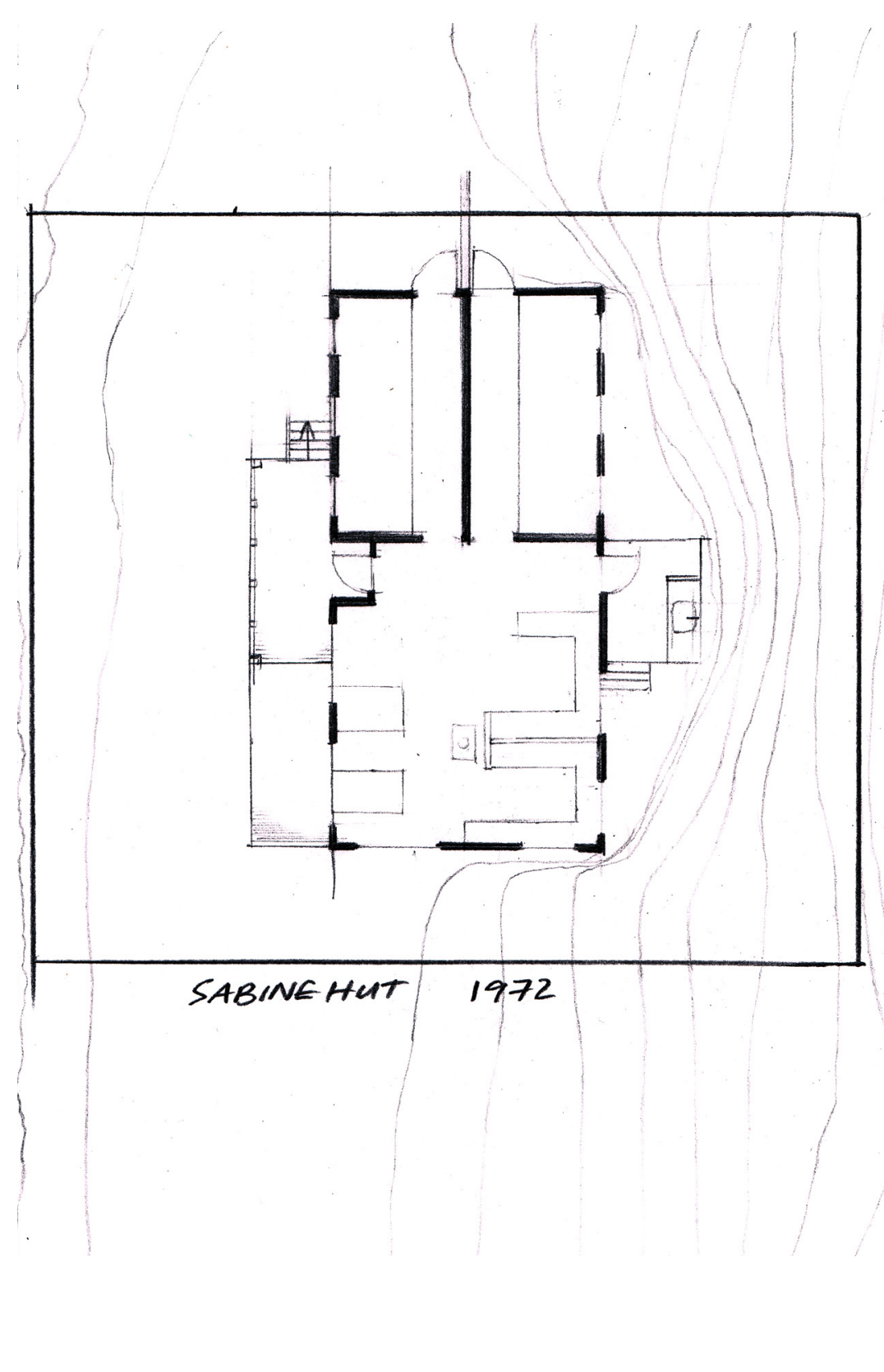

Figure 2.13

Sabine Hut plan, 1:20. The only case-study that has separate sleeping spaces that can be shut off from the living and dining area. A front deck provides shelter and a wet/dry area but is often unused because of the sandflies. 


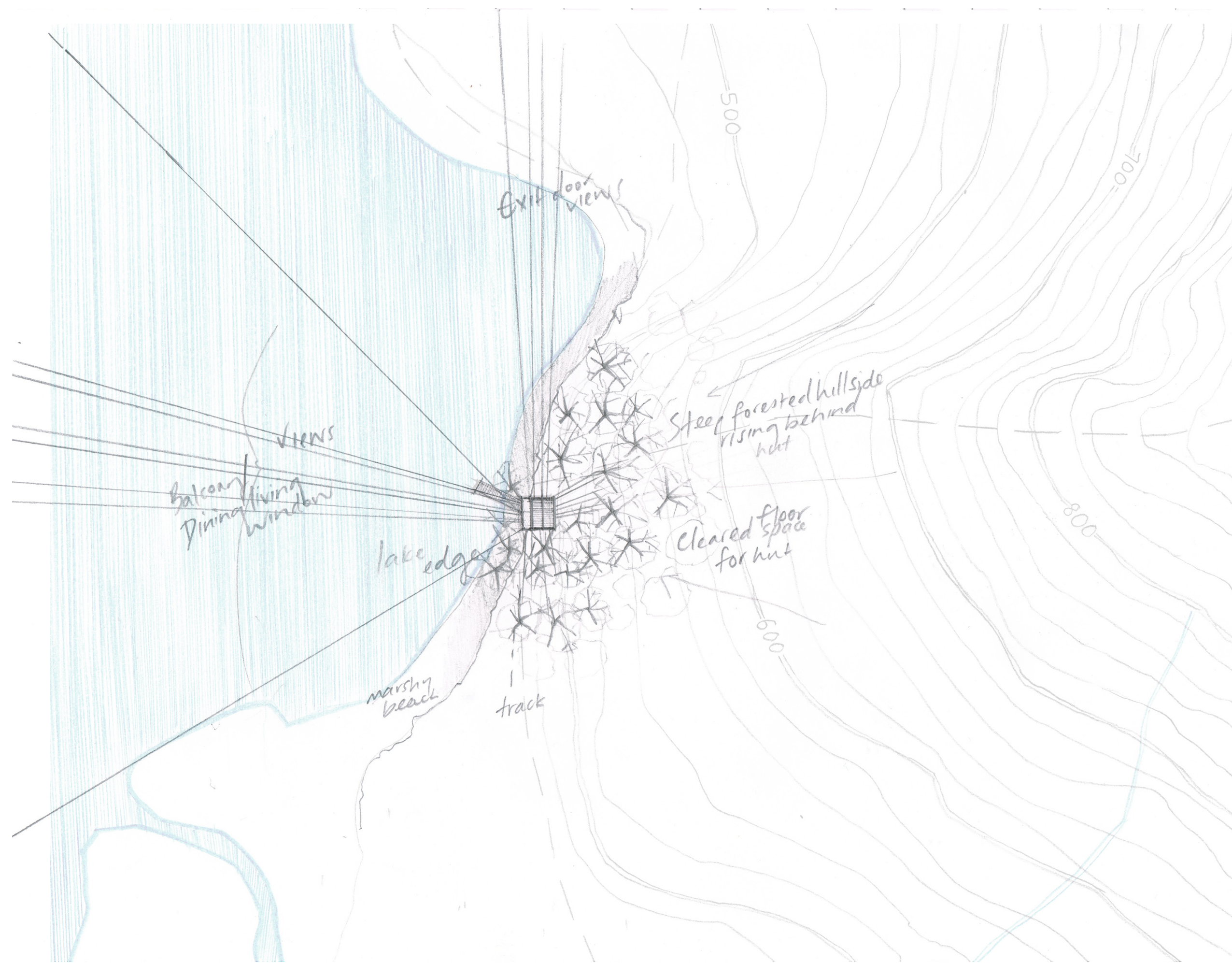

Figure 2.14

Plan analysis of Sabine Hut looking at the relationship between the hut and the Lake Rotoiti setting. Scale 1:1000
Typically Department of Conservation huts are located in clearings in the bush with open tussocky grasslands. This example is different and is nestled amongst the trees right on the edge of the lake; again only windows link the hut to the outer context. 


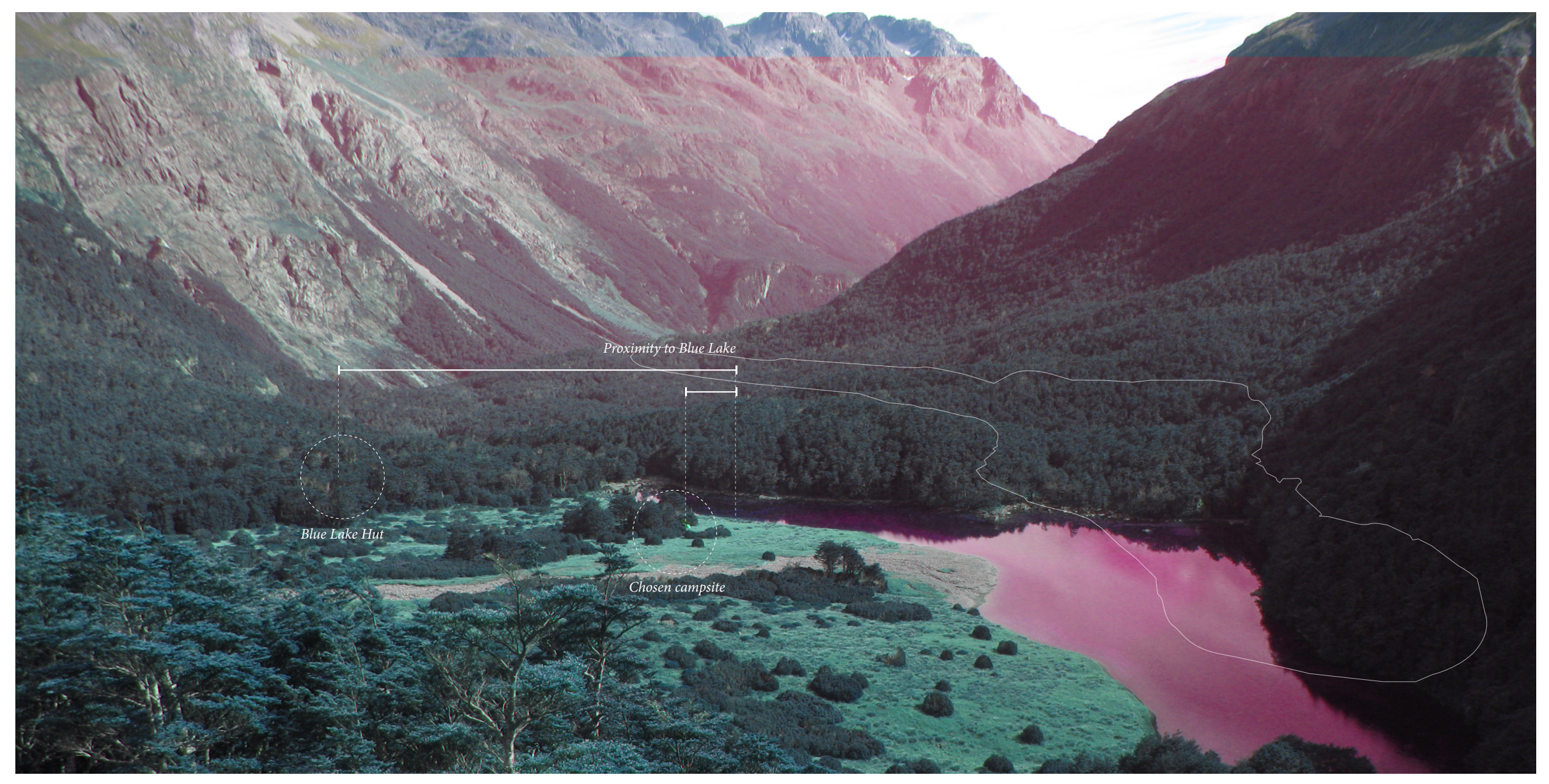

\section{Figure 2.15 (Above)}

This diagram compares between hut siting and tent siting at Blue Lake in Nelson Lakes National Park. A tent can be sited in response to conditions and in fine weather located right beside the lake to take advantage of the beautiful scenery. Whereas the hut is removed from the dramatic context.

\section{Figure 2.16 (Right)}

Tent and tent-fly shelter options, both semi-permanent alternatives to hut accommodation. The tent-fly especially maintains a constant open
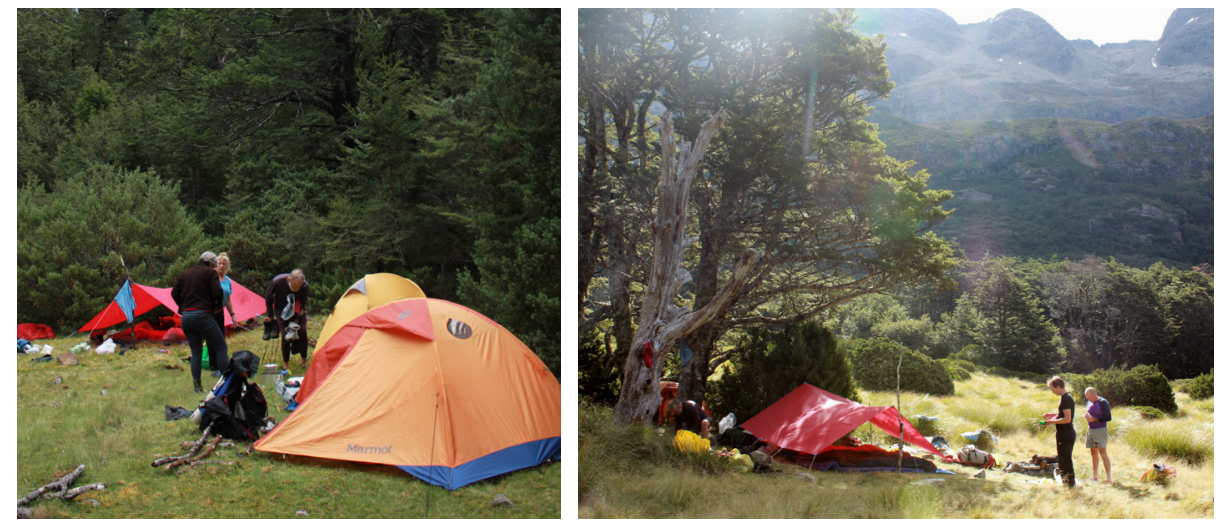
relationship with the surrounding environment so the occupants are always aware and connected with the temporal conditions.

(authors own photographs) 


\section{Wilderness Architecture Case Study - Tom Kundig}

Tom Kundig's works were selected as precedents as he commonly sites buildings in areas where nature dominates the landscape. Each precedent reveals Kundig's sensitive treatment of the relationship between wilderness and architecture. Kundig's designs are not subservient to the landscape but built to respond to a dominant element that the determines the occupants experience of wilderness. Kundig in his design phase responds to the necessities of site, such as raising the buildings off the flood plain, the need for security, and consideration of weathering materials in the extreme conditions (refer to Figure 2.17).

This equal attention to practical requirements of site, and experiential qualities of the architecture raises parallel questions about the critical design issues at Ngapotiki reserve site.

They are outlined as follows:

- The design must be able to withstand extreme winds

- Additional adverse weather conditions, rain, hail, etc

- Safety/Security against property damage

- It must be low cost/affordable

Delta Shelter is relevant to the design research as this building has a very small footprint but a large floor space and 'glamorous' living. Olsen Kundig did the interior decoration and the simple, clean and mechanical aspects of the space continually interact with the outside through moving doors, windows, and walls.

This is also a relevant case study for security as this is one of the considerations that is applicable to Ngapotiki Reserve. This is especially due to the four-wheel drive access bringing a wide demographic of people to the site and therefore increasing the potential for property damage.

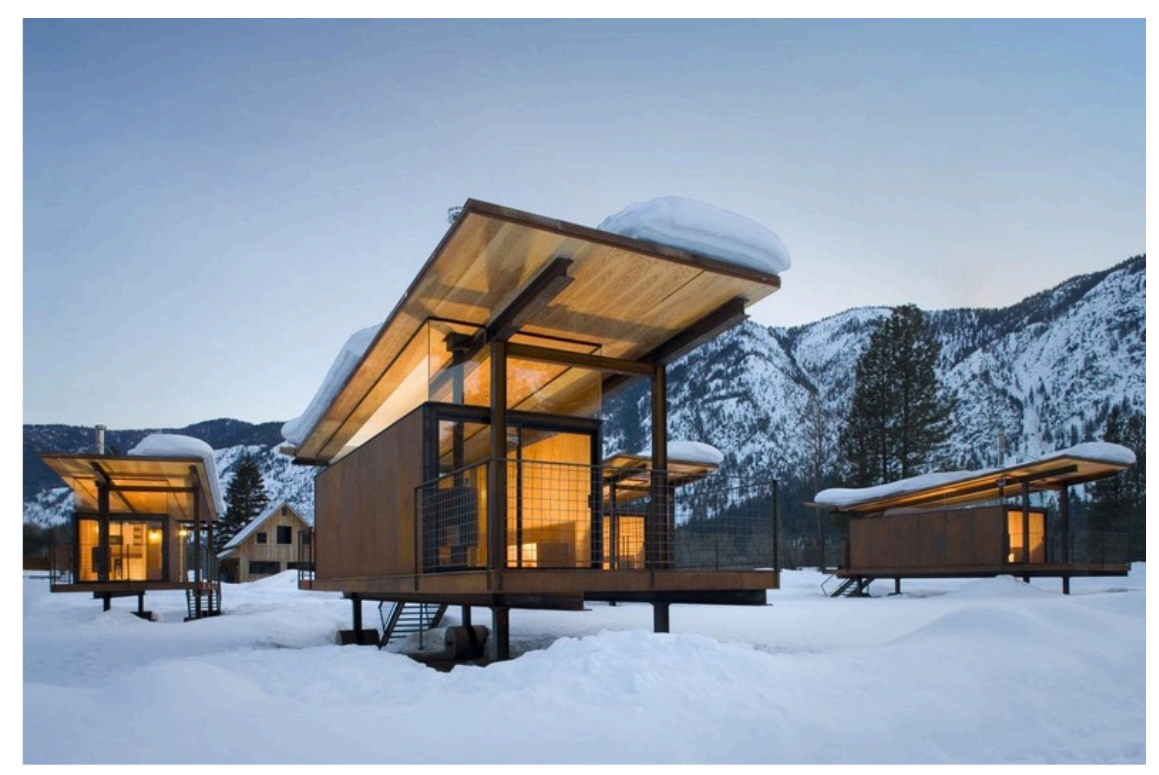

Figure 2.17

Delta Shelter (2005) - Olsen Kundig Architects ${ }^{44}$
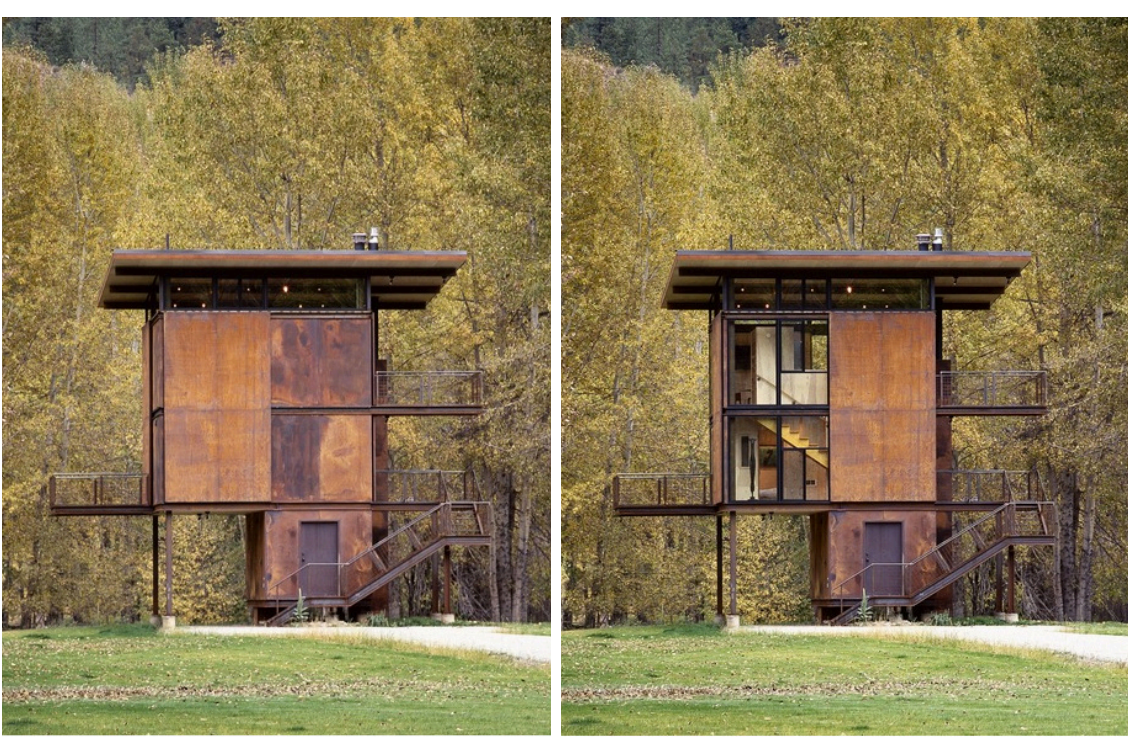

Figure 2.18

Rolling Huts (2007) - Olsen Kundig Architects ${ }^{45}$ 
Affect Case Studies - Weather Art + Architecture

Affect can be deemed as uncontrollable and unpredictable as the changing weather conditions. A selection of work from the practices of art and architecture were analysed to understand the approach for the translation of an uncontrollable condition (weather) into an experience to which the audience can relate. Each precedent 'dramatises' the weather condition by the addition of a material, system, or environment to isolate and heighten the experience and potentially cause affect.

\section{Spring Wind House (2013) - Architecture Uncomfortable Workshop ${ }^{49}$}

This precedent is designed by an architecture firm but is arguably sculptural in practice (refer to Figure 2.10). This relationship between sculpture and architecture is an important boundary which I investigate further throughout this thesis. As explained in the glamour section and in accordance with the affect research; sculpture is removed from practical necessities for occupation so has the potential for a person to have a solely experiential interaction. I wanted to explore the relationship between sculptural and architectural space further by modelling and beginning to inhabit the space. Through adding functional elements to the Wind House we can see the interplay between the fluid outer skin which responds to the wind, and the furniture and people inside (refer to Figure 2.11).

Kunsthaus Bregenz (1997) - Peter Zumthor, ${ }^{50}$ (refer to appendix)

Rainy|Sunny (2008) - Mount Fuji Architects, ${ }^{51}$ (refer to appendix)

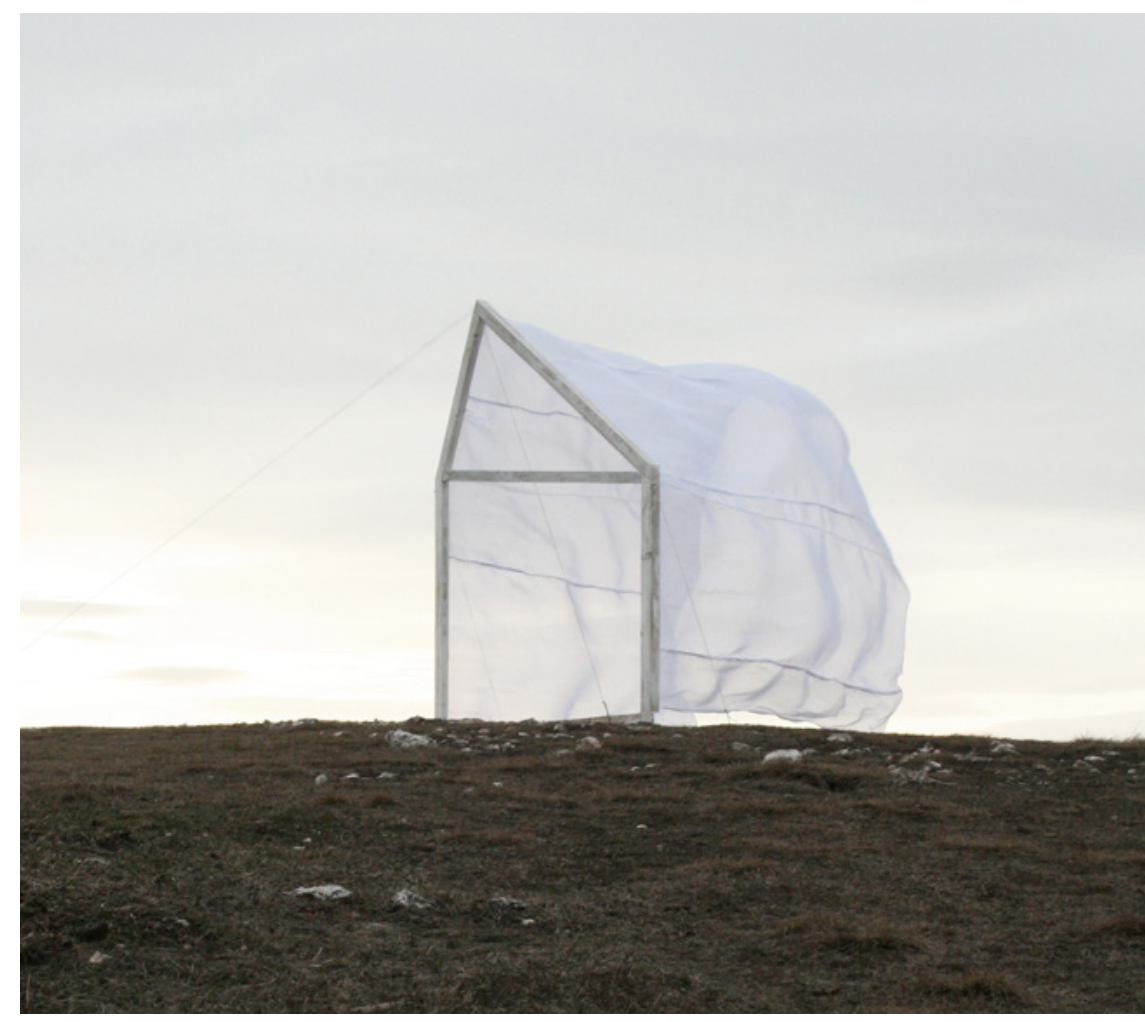

Figure 2.19

Spring Wind House video footage. (click image to begin video)

This shelter is an exploration of the symbolic and poetic expression of the spring wind. It removes all forms of practical function or programme and begins to explore the boundary between architecture and sculpture. This case study is a simple clear example of a purely interactive and beautiful response to site and site conditions. This house creates a multisensory experience that will be memorable because of its removal from the societal norm. 

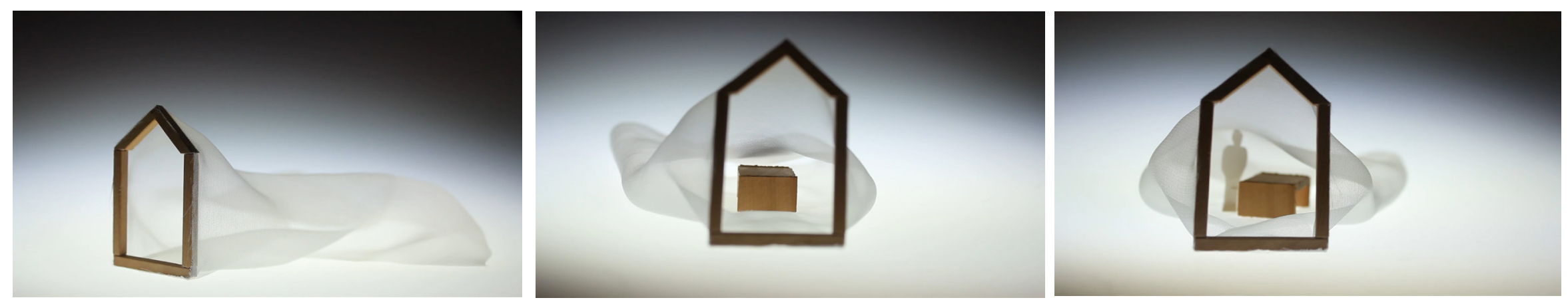
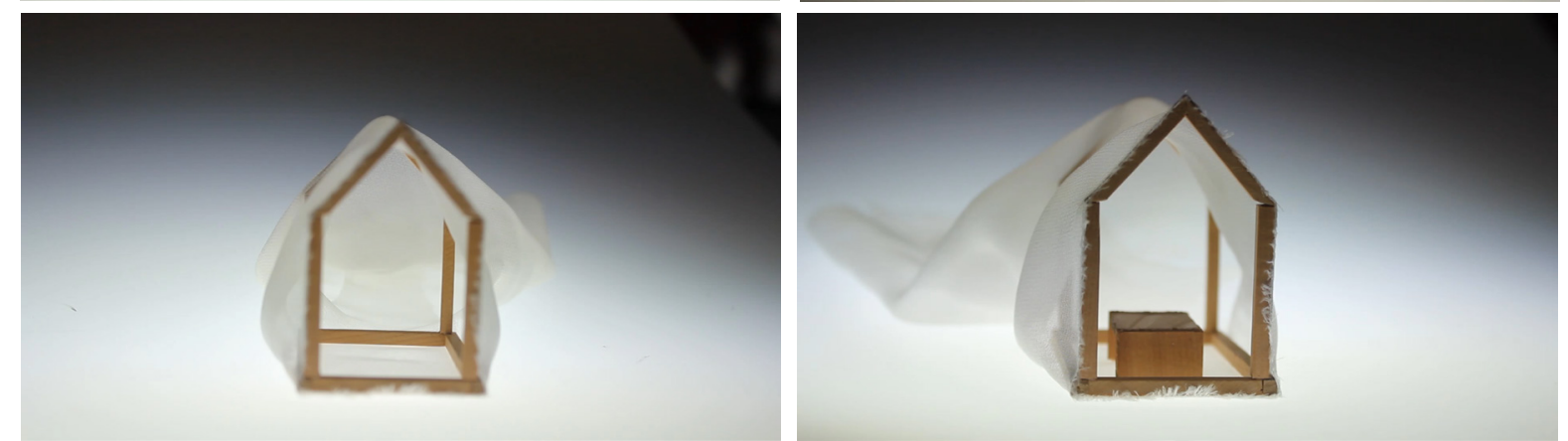

Figure 2.20

Spring Wind House modelling tests and video analysis.

Analysis of the videos shows that the sole arch allowed more movement and interaction between the wind and the architecture. It also was still functional when occupied but on a limited basis. The skin of the house came into contact with the people and furniture on a repeated basis and prolonged occupation of such a space could be draining.
Whereas, with the addition of some archetypal structure the movement become more limited, and therefore less sculptural. The experience becomes more invigorating and the space would be able to be occupied for an extended period of time. Also, increasing the size of the structure would allow a larger free space in the centre so the more fluid skin movement would create a totally immersive space but comfortable occupation of the building. 
The next four case studies are art installations that alter the perception of weather by manipulating space and time through mechanical or computational controls. This sculptural translation of an everyday experience into a state that causes a heightened awareness is parallel to the architectural aim of this thesis to connect people to their surrounding environment.

Kinetic Rain (2012) - ART +COM

Manta Rhei (2012) - ART +COM ${ }^{53}$, (refer to appendix)

Rain Room (2012) - rAndom ${ }^{54}$

Swarm Study I (2010) , III (2011) - rAndom ${ }^{55}$

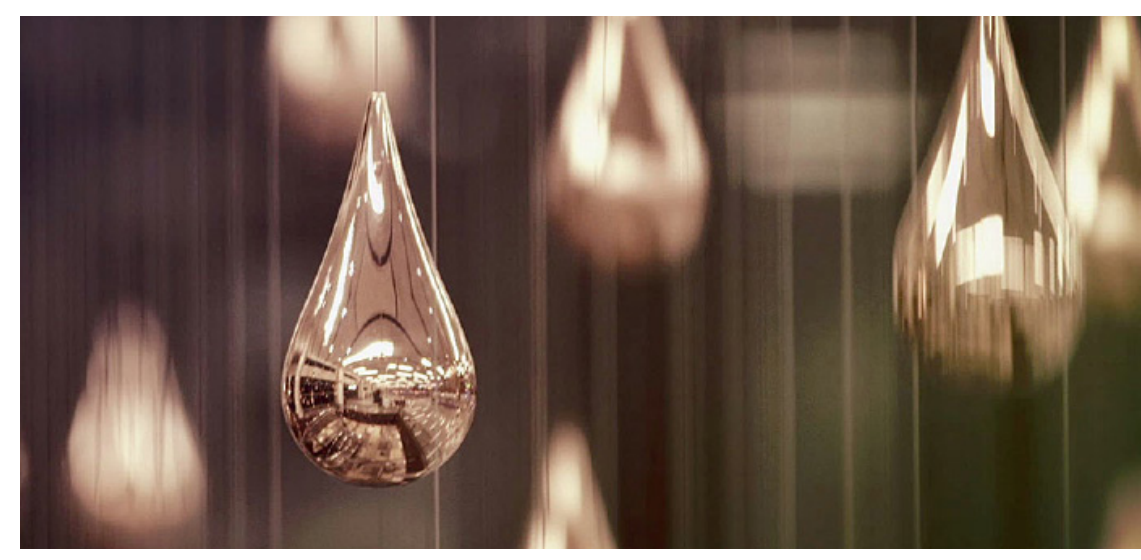

Figure 2.21

Kinetic Rain (2012) - ART +COM ${ }^{47}$

A sculptural installation in the Singapore Changi Airport manipulates how weather moves within space and time by slowing the movement of metallic reflective raindrops into a rhythmic choreographed display. The slow motion creates a moment of awareness of minute details that would not normally be noticed such as the small scenes that are visible in each droplet.

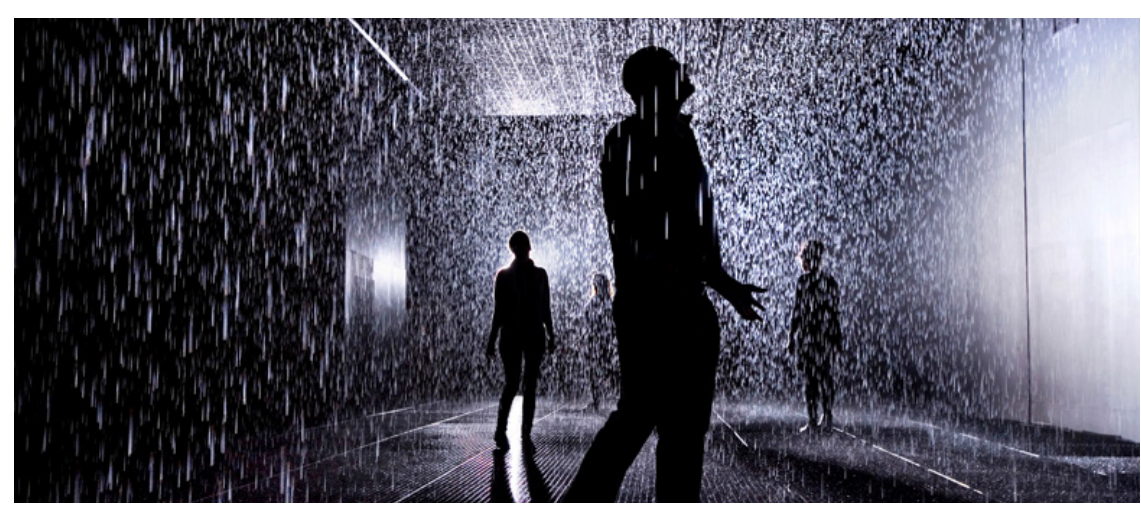

Figure 2.22

Rain Room (2012) - rAndom ${ }^{49}$

Rain room explores human behaviour and interaction using light and movement. A room of falling water confronts the participant but as they slowly move through space a path opens up in response to their movements to allow them to observe, interact with the rain without getting wet. This challenges spatio-temporal expectations to engage the participant. ${ }^{49}$

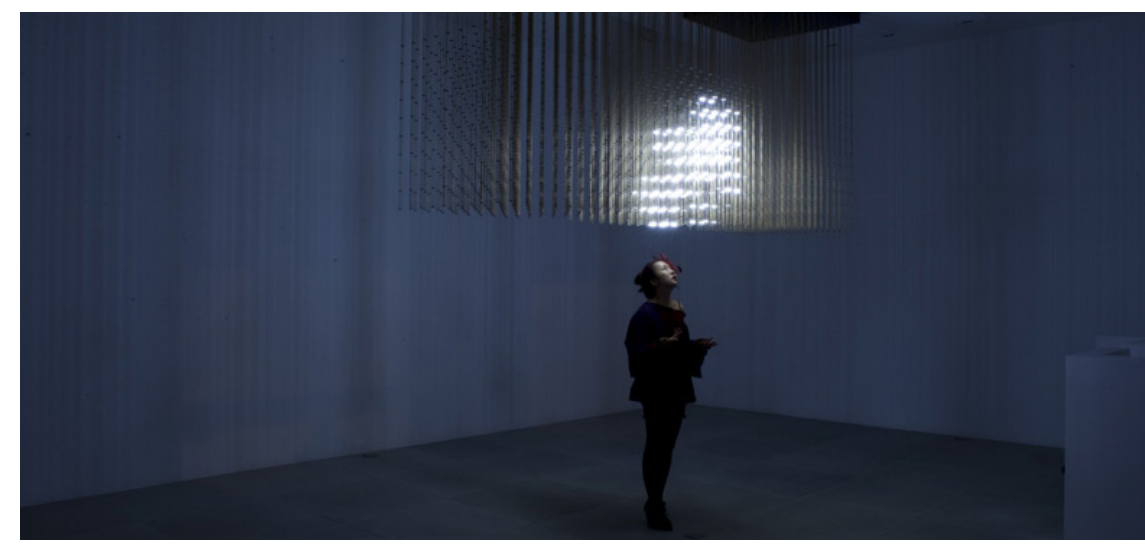

Figure 2.23

Swarm Study I (2010), III (2011) - rAndom ${ }^{50}$

The emulation of the collective behaviour of a group of birds flying is translated into points of lights responding in "swarm-like formations". 50 An interactive relationship is created by sensors which connect the birds movement to the participants movement as they navigate the room. This installation allows a person to interact closely with a rare natural experience. 
The two final affect case studies from Charles Sowers Studios combine sculptural installations and architecture to form interactive systems that are controlled by the weather to emphasise a buildings relationship to its surroundings. Sadly these examples are not integrated into the building design and still act as installations separate to the internal function and programme.

Wave Wall (2006) - Charles Sowers Studios ${ }^{56}$

Windswept Installation (2011) - Charles Sowers Studios ${ }^{57}$

In conclusion this selection of affect related case studies provide examples for how to translate natural conditions into a new state to increase audience impact. A number of different design approaches are used in these precedents, such as:

Mechanical translation of a natural phenomena
(Kinetic Rain, Manta Rhei)
Computational control of a simulated natural
phenomena (Rain room, swarm study)
Carefully designed objects or a series of objects
that interact with the conditions to create an effect
(Windswept installation, Wave Wall)
Materiality and form that allows freedom of control to
act with conditions (Spring Wind House)

The idea of dramatizing the wilderness gained from this context is explored in the Phase One Design Tests.

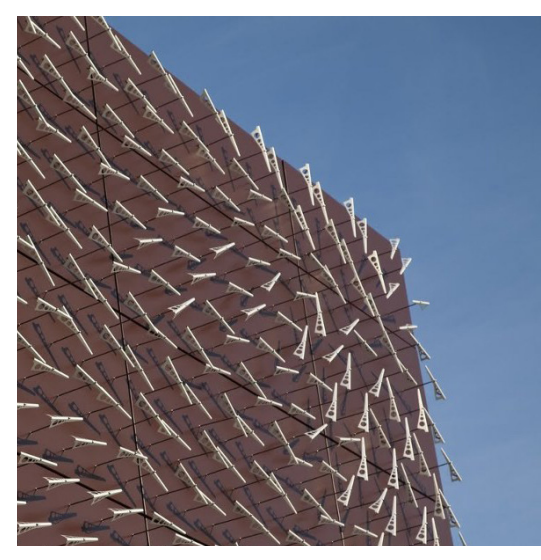

Figure 2.25

Windswept Installation (2011) Charles Sowers Studios ${ }^{51}$

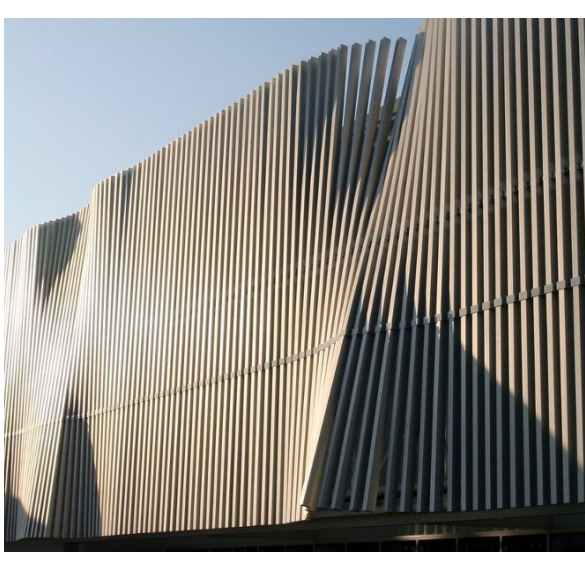

Figure 2.24

Wave Wall (2006) - Charles Sowers Studios ${ }^{52}$
The Wave Wall installation is designed to display how wind gusts, "rippling and swirling through the sculpture, visually reveal the complex and ever changing ways wind interacts with the building and the environment". ${ }^{51}$ Both of these examples explore wind as a largely invisible phenomenon.

In Wave wall mechanical systems create this undulating surface of windactivated pendulums which interact with the wind to form a dynamic façade of this building. When the wind is greater than 15 knots the magnets that couple members to create the undulation are broken apart and a chaotic frenzy of movement ensues. ${ }^{52}$ This kinetic façade presents another of endless methods of focussing attention to the interplay between environmental conditions and architecture. The downfall with this installation is it lacks the unity with the complete building system. Despite this the focus on mechanical systems as opposed to the digitally controlled equivalents is closer to the intention for this thesis to raise awareness of the environment. The most important thing about these case studies is that they reveal the interaction between wind and the facade of the building, an interaction that is usually resisted through the structural design of the building. 
Non-Static Case Studies - Movement Systems

Two main non-static case studies were analysed for the complexity in their creation and mechanical systems as well as their design approach and potential for development.

\section{Strandbeests (1990) - Theo Jansen ${ }^{58}$}

Theo Jansen is an artist that breaks the boundaries of naturally powered kinetic movement through the creation of animals that roam the beaches of Holland. He forms them out of complex systems in a mode of biomorphology; imitating the elements of the human body to create responsive and interactive beings that are powered solely by wind. Jansen creates this system through an evolutionary design methodology where he uses a combination of computer modelling and physical prototyping to analyse and attempt to create the most efficient system. The animals are allowed to 'reproduce' when the are successful and the computer generates a plethora of new design alternatives from that current strand.

This case-study is relevant to the non-static as Jansen explores the interactive relationship between a man-made object and wind. He creates systems that are completely sustainable and ideally endlessly cyclic. A similar interaction between the wind and other natural rhythms on site at Ngapotiki Reserve will occur to create a changeable non-static architecture. The rigorous process of combined digital and physical modelling to create the most successful system in a small amount of time is a relevant and efficient design development strategy.

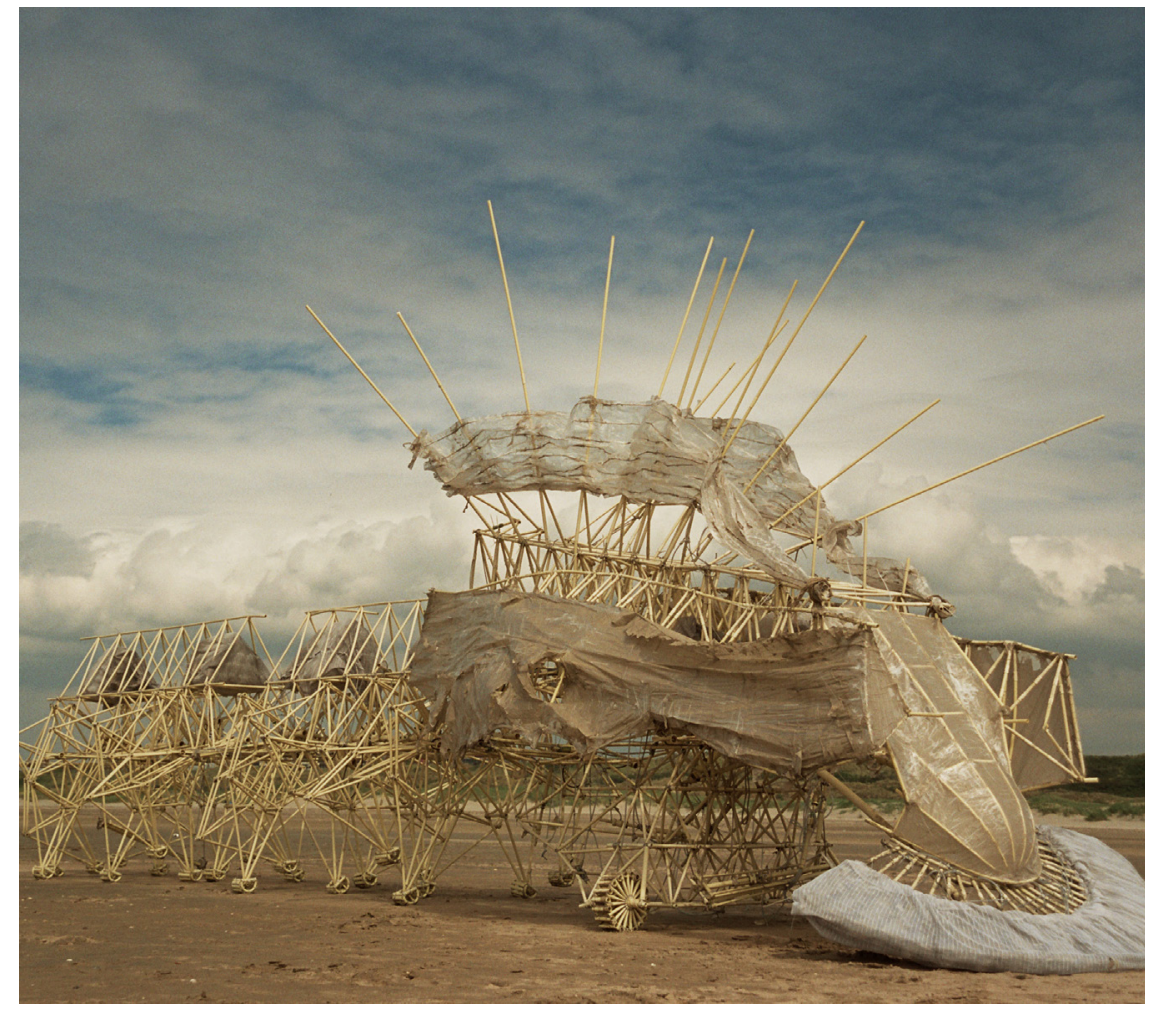

Figure 2.26

Strandbeests (1990) - Theo Jansen, ${ }^{55}$ (click on image to follow to video link) 
Stick weaving (2007) - Bradford Hansen-Smith ${ }^{59}$

Bradford Hansen-Smith is a sculptor from Chicago, Illinois, whose work is focussed on geometry; in particular the spatial patterns of movement. He has created multiple transformable patterns in different materials and with different base formations. One such sculpture is the stick weave; this consists of weaving 10-inch bamboo skewers joined with short pieces of rubber tubing. ${ }^{60}$ The transformable function of the stick lends itself appropriately to non-static architecture. Bradford Hansen-Smith started a business to develop this technique for movement systems and designed robotic prototypes, emergency shelters and other deployable uses. He spent time researching the origins of this method of design and traced it back to Buckminster Fuller who describes a static non-moving version of the weave in "Synergetics" or "Synergetics Two". The business collapsed and he turned to education as a method of teaching that extended the potential of traditional geometric models. ${ }^{61}$ Hansen-Smith reinforces the potential of this transformable structure and concludes with encouraging others to develop the weave further.
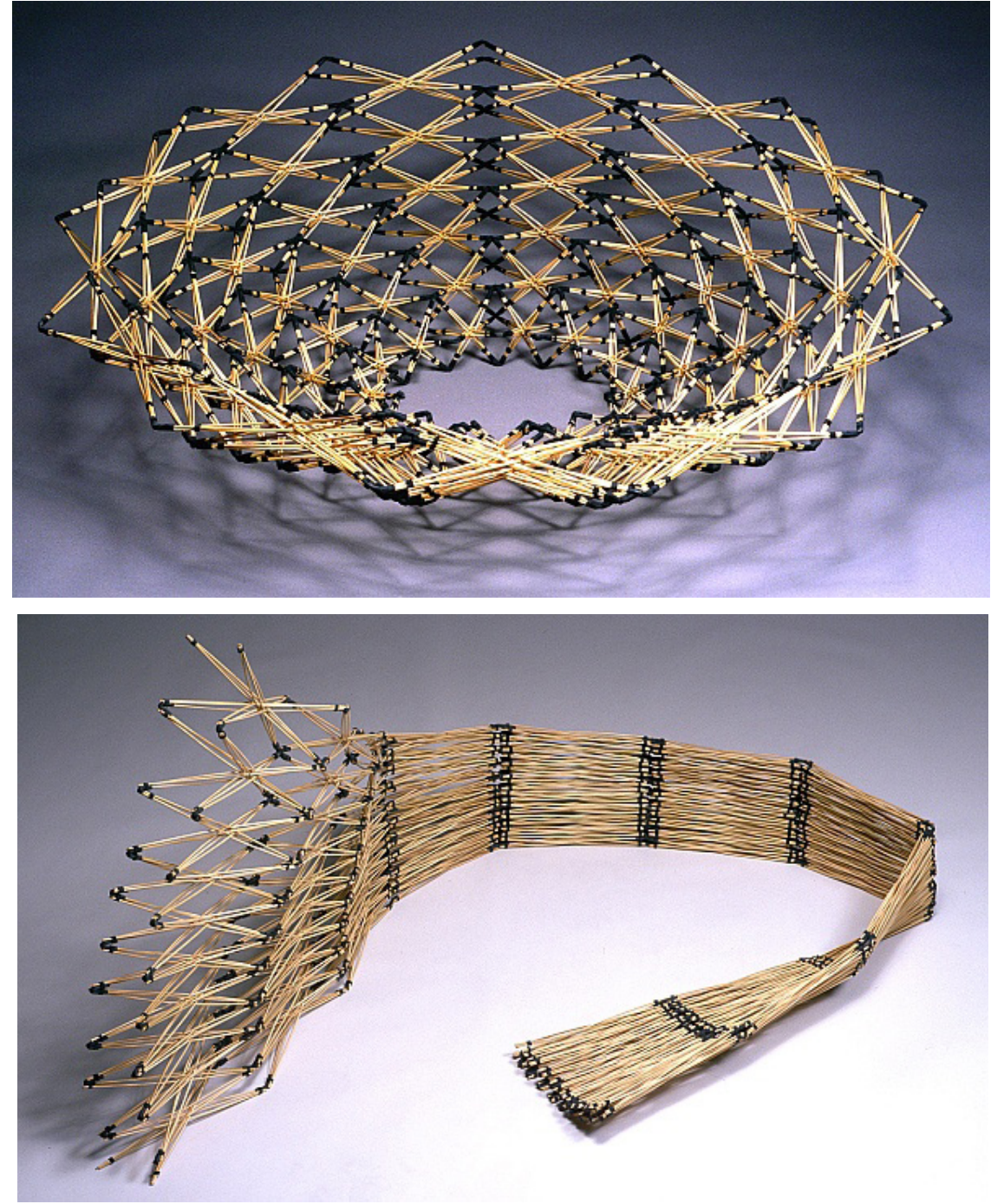

Figure 2.27

Bradford Hansen-Smith's Stick weaving examples 
A series of precedents were analysed for their material use, temporality and relevance to the stick weave structural design. Each of these precedents informed the design process by identifying the current material research context and therefore heightening personal design expectations. The following case studies show successful methods of detailing and treatment of natural materials, a strand of research that is critical to the resolution of this design enquiry.

Passive House (2009) - Karawitz Architecture ${ }^{62}$, (refer to appendix)

Folded Bamboo + Paper House (2008 - unbuilt) - Tang + Yang Architects ${ }^{63}$ (refer to appendix)

Bamboo Wing (2010) - Vo Trong Nghia ${ }^{64}$

Ecological Children Activity and Education Centre (2008-09) - 24H > Architecture $^{65}$
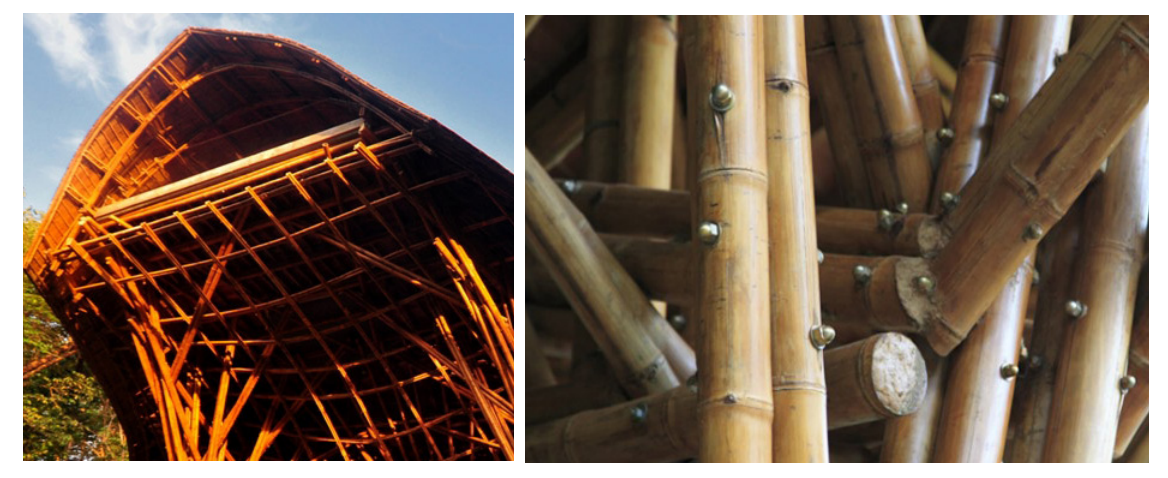

Figure 2.29

Ecological Children Activity and Education Centre (2008-09) - 24H > Architecture. $^{58}$

This is a clever example of a semi-permanent structure with a simple construction method and dome form. This example is most applicable to my research in the speed of construction and the use of vernacular and local materials including bamboo. The multiple screw fixed structural members 60 and laminated poles were researched as a potential method of fixing.

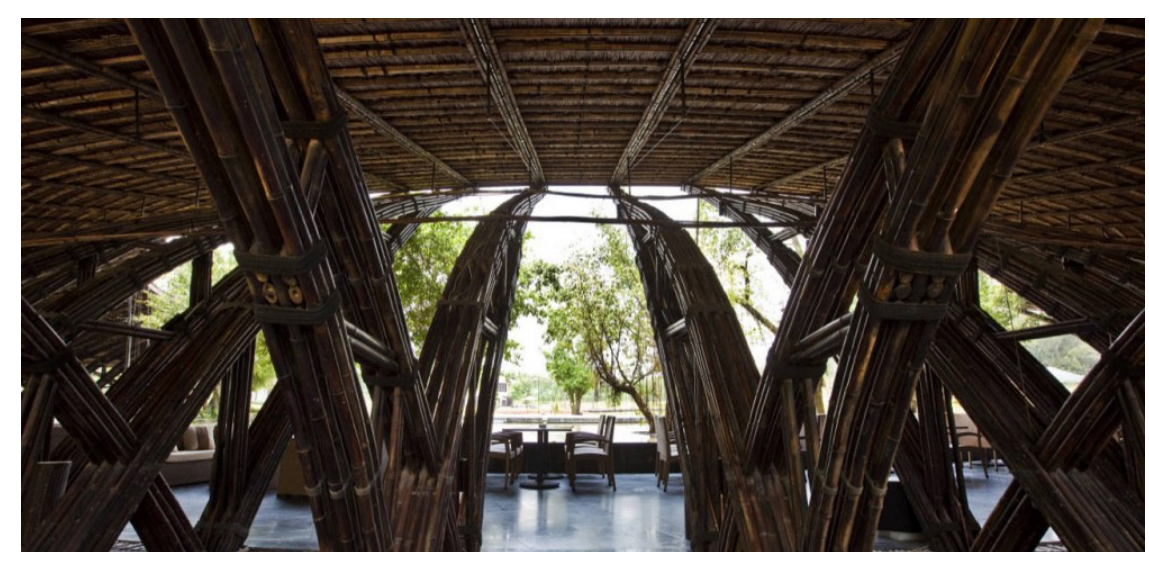

Figure 2.28

Bamboo Wing (2010) - Vo Trong Nghia. ${ }^{57}$

Bamboo Wing is the first large scale project that tests the use of bamboo as the only structural material. Vo Trong Nghia, the architects have created a region specific and ecologically responsive design that is cheap to construct and beautiful in form. The slowly arching supports are created through combining many bamboo poles into one structural member. This is as a reaction to the sheer scale of the design and not being able to source bamboo that is large enough for a single pole as each member.

This case study was selected as I began to research methods of fixing bamboo together without drilling through it. Vo Trong Nghia uses a binding system similar to cane furniture, and boat cord fixing called whipping that is structurally strong enough for a building of this size. I experimented with this binding system as explained in Chapter 5, Design Phase Three. 


\section{Material Case Studies - Skin Precedents}

Additional material precedents were comparatively analysed for the relationship between skin, structure and transformation. The studies that involve movement are especially important to guide the design of the membrane to stick-weave relationship. Initially I wanted to explore the idea of a transformable skin but due to the limited time for this thesis and the high level of technical detailing required for weather-tightness this strand of research was not continued. The following precedents still give some idea of the current research in this field and are described in the appendix.

DAL Canopy Design (2011) - Digital Architectural Lab ${ }^{67}$, (refer to appendix)

Expandable Surface Pavilion (2011) - Pablo Zamorano, Nacho Marti + Jacob Bek ${ }^{68}$ (refer to appendix)

Dragon Skin Pavilion (2012) - Emmi Keskisarja, Pekka Tynkkynen + $L E A D^{69}$, (refer to appendix)

\section{Structural Case Studies - Weave Precedents}

This final selection of case studies presents a view of similar unconventional structural systems to the stick-weave design. Most do not engage responsively with their environment but instead prioritise the use of new technologies and maximising materials for their intrinsic potential.

Static Truss (2005) Georgia Klonizaki + Dina Nikolaidou ${ }^{70}$

SUTD Library Pavilion (2013) - City Form Lab L1 $^{71}$

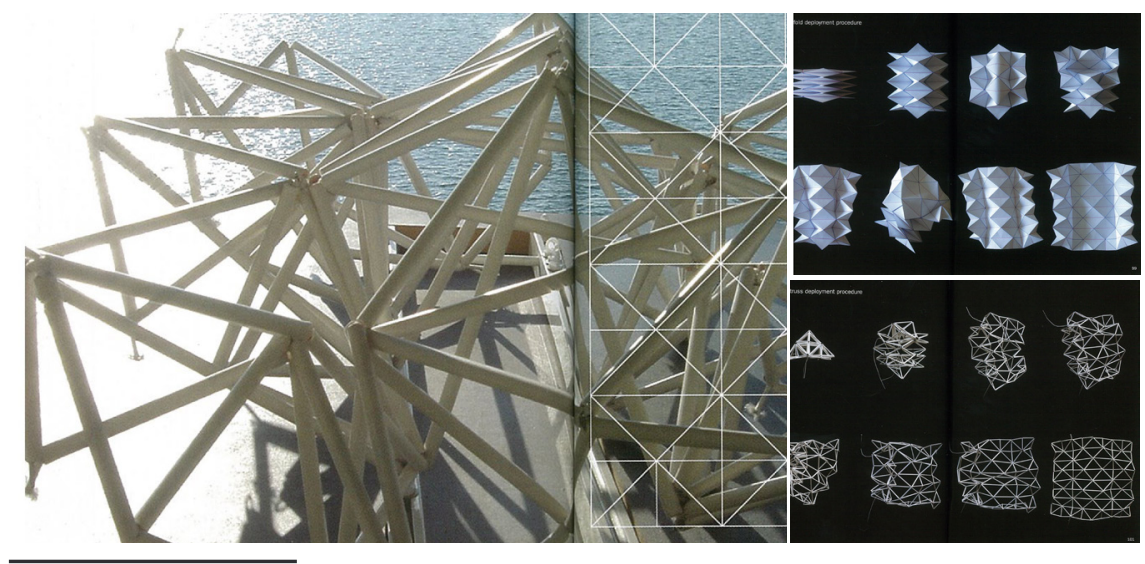

Figure 2.30

Static Truss (2005) Georgia Klonizaki + Dina Nikolaidou ${ }^{70}$

Static truss applies a similar mode of stick weaving to Bradford HansenSmith's example but with different intentions. This case study takes a transformable folded paper system and translates it into structural members. Although this is called Static Truss the nature of the design process means it could also be flexible. The complexity and transformability all lies in the jointing. This example is included in the precedents section as it is a similar structure to the stick-weave that was developed to form the final design. The design process also pushed me to think of other structural systems that could become transformable structures. 

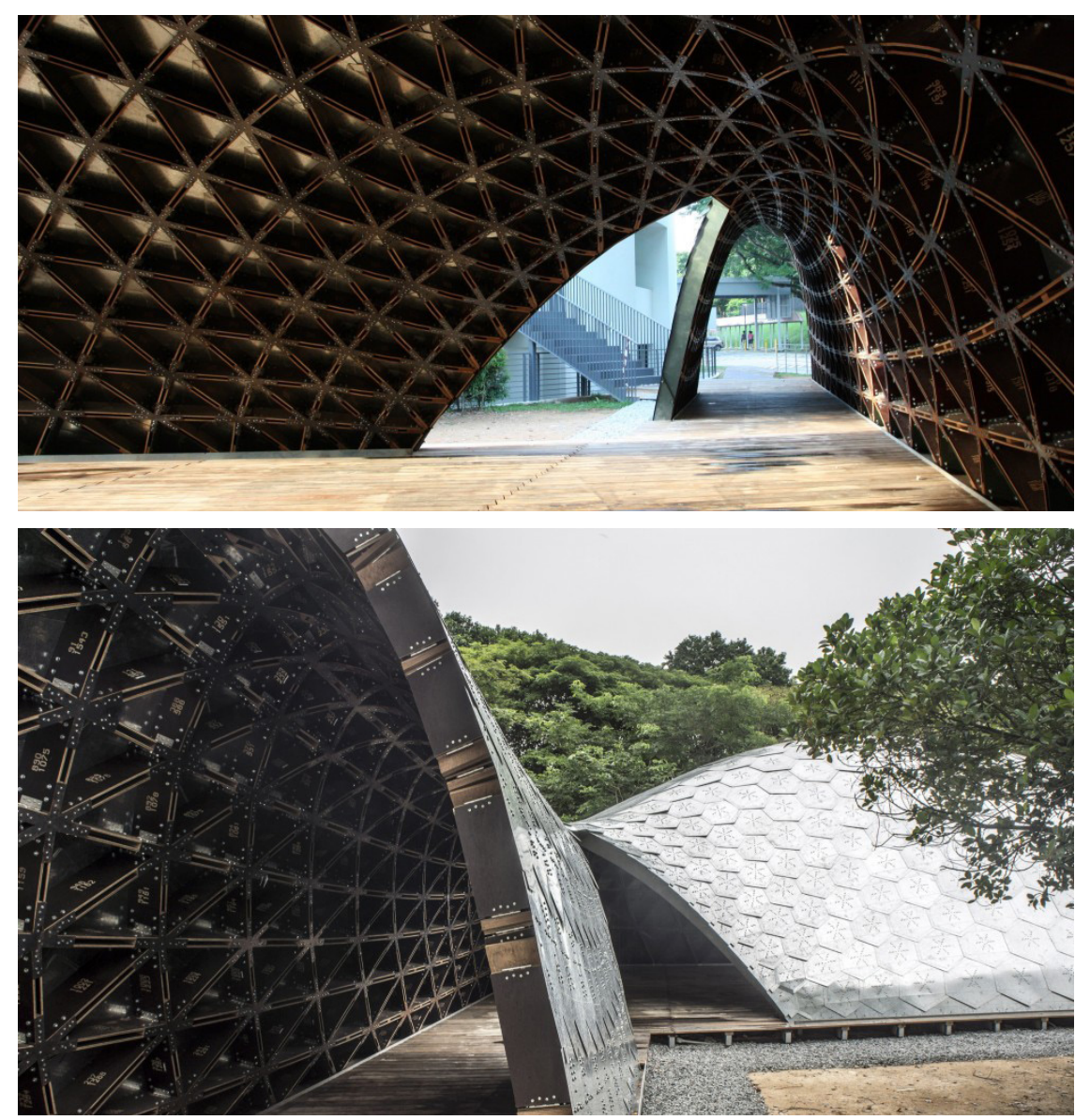

Figure 2.31

SUTD Library Pavilion (2013) - City Form Lab ${ }^{63}$

This catenary structure is a lightweight and strong design that was designed parametrically to save time and money. The digital modelling allowed a more complex curve that physical design generation and streamline the construction process through the use of CNC machines and readily available materials. The relationship between rigid structure and skin hides the complexity and beauty in the timber design and instead creates a form and mass based connection to the surrounding buildings. Although this is successful it is also a critique as I believe it would be more visually appealing from the outside if there was more indication of the detailing within.
Pavilion design has the added luxury of an often-undefined program and therefore boarders the art context of sculpture. Yet in these examples the process, materiality and research involved begin to question if these two historically distinct fields are still so separate.

ICD/ITKE Research Pavilion (2013) - University of Stuttgart, Faculty of Architecture and Planning ${ }^{72}$

Wood Pavilion (2010) - Wing Yi Hui + Lap Ming Wong ${ }^{73}$, (refer to appendix)

Lincoln Park Zoo South Pond (2010) - Studio Gang Architects ${ }^{74}$, (refer to appendix)

O-Strip Pavilion (2011) - Tongji University Team ${ }^{75}$

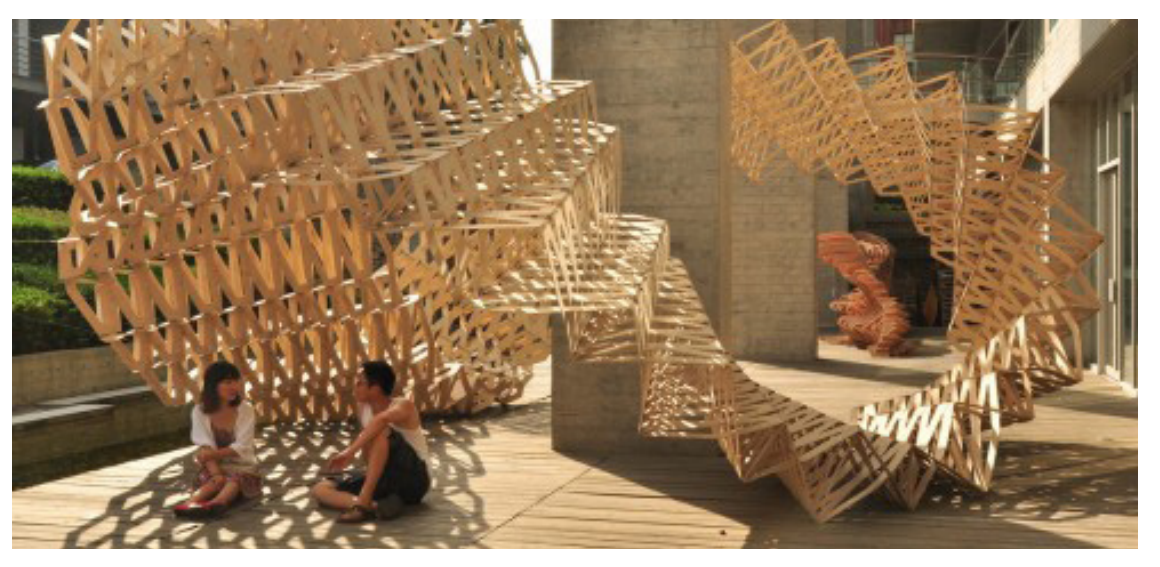

Figure 2.32

O-Strip Pavilion (2011) - Tongji University Team ${ }^{67}$

This flexible timber structure uses a similar prefabricated weaving technique to the stick-weaving to form a new structural re configuration of the truss. This design reinforces the use of parametric design technology to create systems for ease of construction and creativity of design. This pavilion is a static installation with the o-strip design naturally maintaining the structures form. 


\section{References}

1. Bronwyn M. Newton, John R. Fairweather, and Simon R. Swaffield,

"Public Perceptions of Natural Character in New Zealand: Wild Nature Versus Cultured Nature," New Zealand Geographer 58, no. 2 (2002): 17, doi:10.1111/j.1745-7939.2002.tb01632.x; William Cronon, "The Trouble with Wilderness: Or, Getting back to the Wrong Nature," Environmental History 1, no. 1 (1996): 7; R. Bruce Hull, David P. Robertson, and Angelina Kendra, "Public Understandings of Nature: A Case Study of Local Knowledge about 'Natural' Forest Conditions," Society \& Natural Resources 14, no. 4 (2001): 326.

2. Mick Abbott, "Designing Wilderness as a Phenomenological Landscape: Design-Directed Research Within the Context of New Zealand's Conservation Estate" (Lincoln University, 2008), 111, http://researcharchive. lincoln.ac.nz/dspace/handle/10182/1026.

3. Newton, Fairweather, and Swaffield, "Public Perceptions of Natural Character in New Zealand," 24

4. Cronon, "The Trouble with Wilderness," 7; Hull, Robertson, and Kendra "Public Understandings of Nature," 327; Phil Macnaghten and John Urry, Contested Natures, vol. 54 (Sage, 1998), 95; Jonathan Murdoch, "Inhuman/nonhuman/human: Actor-Network Theory and the Prospects for a Nondualistic and Symmetrical Perspective on Nature and Society, Environment and Planning D 15 (1997): 732.

5. Bruce Braun and Noel Castree, Remaking Reality: Nature at the Millenium (Routledge, 1998), 34; Newton, Fairweather, and Swaffield, "Public

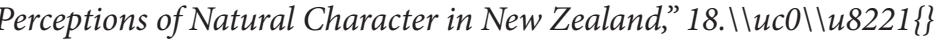
18.\}","plainCitation":"Bruce Braun and Noel Castree, Remaking Reality: Nature at the Millenium (Routledge, 1998

6. Newton, Fairweather, and Swaffield, "Public Perceptions of Natural Character in New Zealand," 25

7. Ibid., 19

8. Abbott, "Designing Wilderness as a Phenomenological Landscape: DesignDirected Research Within the Context of New Zealand's Conservation Estate," 17-20.

9. Ibid., 17.

10. Eric Shouse, "Feeling, Emotion, Affect," Journal of Media and Culture 8, no. 6 (December 2005), http://www.journal.media-culture.org.au/0512/03shouse.php.
11. Nigel Thrift, "Intensities of Feeling: Towards a Spatial Politics of Affect," Geografiska Annaler: Series B, Human Geography 86, no. 1 (2004): 24 doi:10.1111/j.0435-3684.2004.00154.x.

12. Shouse, "Feeling, Emotion, Affect."

13. Ibid

14. Thrift, "Intensities of Feeling," 60-64.

15. Ibid., 60

16. Ibid., 64

17. Ibid., 61

18. Silvan S. Tomkins, Exploring Affect: The Selected Writings of Silvan S Tomkins (Cambridge: Cambridge University Press, 1995).

19. Eve Kosofsky Sedgwick and Adam Frank, Touching Feeling: Affect, Pedagogy, Performativity (Duke University Press, 2003).

20. Tomkins, Exploring Affect.

21. Sedgwick and Frank, Touching Feeling, 19.

22. Thrift, "Intensities of Feeling," 62.

23. Ibid., 70

24. Ibid., 74

25. Rodolphe El-Khoury, Christos Marcopoulos, and Carol Moukheiber, The Living, Breathing, Thinking, Responsive Buildings of the Future (London: Thames \& Hudson, 2012), 14.

26. Ibid., 13.

27. Michael Fox and Miles Kemp, Interactive Architecture (Princeton Architectural Press, 2009), 12.

28. Ibid., 13; El-Khoury, Marcopoulos, and Moukheiber, The Living, Breathing, Thinking, Responsive Buildings of the Future, 14.

29. Fox and Kemp, Interactive Architecture, 20.

30. Ibid., 169

31. Ibid.

32. Thrift, "Intensities of Feeling."

33. Mehul Bhatt, Frank Dylla, and Joana Hois, "Spatia-Terminological Inference for the Design of Ambient Environments," in Spatial Information 
Theory (Aber Wrac'h, France: COSIT, 2009), 378.France: COSIT, 2009

34. Thrift, "Intensities of Feeling," 61.

35. Elaine Higgleton, Howard Sargeant, and Anne Seaton, eds., Chambers Pocket Dictionary (Edinburgh: Chambers Harrap Publishers, 1997).

36. Ibid., 4 .

37. Edward Brooker and Marion Joppe, "Trends in Camping and Outdoor hospitality-An International Review," Journal of Outdoor Recreation and Tourism 3-4 (December 2013): 1, doi:10.1016/j.jort.2013.04.005.

38. Abraham Harold Maslow and Karen J. Lewis, Maslow's Hierarchy of Needs (Salenger Incorporated, 1987), http://www.researchhistory.org/2012/06/16/ maslows-hierarchy-of-needs/.

39. Naomi Rosh White and Peter B. White, "Travel as Transition: Identity and Place," Annals of Tourism Research 31, no. 1 (January 2004): 201, doi:10.1016/j.annals.2003.10.005; Roger C. Mannell and Seppo E. IsoAhola, "Psychological Nature of Leisure and Tourism Experience," Annals of Tourism Research 14, no. 3 (1987): 324, doi:10.1016/0160-7383(87)901058; Gordon Bultena and Marvin J. Taves, "Tenting on a Park Campground," Conserv. Volunteer 23 (1960): 60-64; Brooker and Joppe, "Trends in Camping and Outdoor hospitality -An International Review," 3.

40. Mannell and Iso-Ahola, "Psychological Nature of Leisure and Tourism Experience," 324.

41. Sjerp de Vries et al., "Natural Environments, Healthy Environments? An Exploratory Analysis of the Relationship between Greenspace and Health," Environment and Planning A 35, no. 10 (2003): 1718, doi:10.1068/a35111; Eeva Karjalainen, Tytti Sarjala, and Hannu Raitio, "Promoting Human Health through Forests: Overview and Major Challenges," Environmental Health and Preventive Medicine 15, no. 1 (March 25, 2009): 2, doi:10.1007/ s12199-008-0069-2; Marc Schweitzer, Laura Gilpin, and Susan Frampton, "Healing Spaces: Elements of Environmental Design That Make an Impact on Health," The Journal of Alternative and Complementary Medicine 10, no. 1 (September 1, 2004): 71-83, doi:10.1089/1075553042245953.\\uc0\\ u8221 \{\}$\backslash i$ Environment and Planning $A \backslash i 0\{\} 35$, no. $10(2003$

42. The British Camping and Caravanning Club, "Get Richer, Feel Better," WebLabs.Modules.ContentPages.IndexPage, The Camping and Caravanning Club, April 15, 2011, http://www.campingandcaravanningclub.co.uk/ newsandevents/get-rich-quick/real-richness-the-list/get-richer-feel-better/.

43. Fox and Kemp, Interactive Architecture, 20.

44. David Blake, "Assorted Wilderness Photography," Google+, January 19,
2010, https://plus.google.com/photos/115458569418877025347/albums/543 0113215124574049 ? banner=pwa\&partnerid $=$ pwrd1.

45. Ibid.; Trippy Tramper, "Tramping: New Angelus Hut," October 31, 2010, http://trippytramping.blogspot.co.nz/2010/11/new-angelushut-30-31102010.html.

46. Kathrin Marks and Stefan Marks, Sabine Hut, February 8, 2011, http:// www.flickr.com/photos/ks_marks/5468587193/; Jan Robertson, Sabine Hut View Nelson Lakes, December 31, 2010, http://www.flickr.com/photos/ backcountrynz/8609196904/; David Noble, "Travers - Sabine Circuit Nelson Lakes National Park, Day6," David Noble, January 10, 2010, http:// www.david-noble.net/NZ/Jan10/TraversSabine/Day6.html.

47. Tom Kundig, "Delta Shelter," Olson Kundig Architects, 2013, http://www. olsonkundigarchitects.com/Projects/38/Delta-Shelter.

48. Tom Kundig, "Rolling Huts," Olson Kundig Architects, 2013, http://www. olsonkundigarchitects.com/Projects/825/Rolling-Huts.

49. Architecture Uncomfortable Workshop, "Spring Wind House," Designboom, accessed March 24, 2013, http://www.designboom.com/readers/springwind-house/.

50. Peter Zumthor, “Kunsthaus Bregenz," ArchDaily, 1997 1989, http://www. archdaily.com/107500/ad-classics-kunsthaus-bregenz-peter-zumthor/.

51. Mount Fuji Architects Studio, "Rainy|Sunny / Mount Fuji Architects Studio," ArchDaily, 2008, http://www.archdaily.com/48381/rainy-sunnymount-fuji-architects-studio/.

52. ART+COM, "Kinetic Rain" Changi Airport Singapore, 2012, http://vimeo. com/45188800.

53. ART+COM, "ART+COM : Manta Rhei," accessed March 25, 2013, http:// www.artcom.de/en/projects/project/detail/manta-rhei/.

54. rAndom, “Rain Room' Installation / rAndom," ArchDaily, 2012, http:// www.archdaily.com/288219/rain-room-installation-random/.

55. rAndom, "Swarm Light - rAndom International," 2010, http://randominternational.com/work/swarm-light/; rAndom, "Swarm Study / III - rAndom International," 2011, http://random-international.com/work/ swarm-study-iii/.

56. Charles Sowers, "Charles Sowers | Wave Wall," Charles Sowers Public Art Works and Science Experiments, 2006, http://charlessowers.com/wave-wall. 
57. Charles Sowers, "Charles Sowers | Windswept," 2011, http://charlessowers. com/windswept.

58. Theo Jansen - The Great Pretender, 2009, http://www.youtube.com/ watch? $v=5$ NOIonPOy-I\&feature=youtube_gdata_player; Theo Jansen, "Strandbeest," Theo Jansen's Strandbeest, accessed January 10, 2014, http:// www.strandbeest.com/theo_cv.php.

59. Bradford Hansen-Smith, "The History of Stickweaving," Stickweaving: The Work of Bradford Hansen-Smith, 2007, http://www.stickweaving.com/ history.html.

60. Ibid.

61. Ibid.

62. Karawitz Architecture, "Passive House," ArchDaily, 2009, http://www. archdaily.com/84165/passive-house-karawitz-architecture/.

63. Ming Tang, "Folded Bamboo + Paper House," ArchDaily, 2008, http://www. archdaily.com/8124/folded-bamboo-paper-house-ming-tang/.

64. Vo Trong Nghia, "Bamboo Wing," ArchDaily, 2010, http://www.archdaily. com/219880/bamboo-wing-vo-trong-nghia/.

65. $24 \mathrm{H}>$ architecture, "Ecological Children Activity and Education Center / 24H > Architecture," ArchDaily, 2007 2006, http://www.archdaily. com/34946/ecological-children-activity-and-education-center-24harchitecture/.

66. Pouya Khazaeli Parsa, "Bamboo Structure Project," ArchDaily, accessed June 26, 2013, http://www.archdaily.com/93922/bamboo-structure-projectpouya-khazaeli-parsa/.

67. Digital Architectural Lab, "DAL Canopy Design," ArchDaily, September 1, 2011, http://www.archdaily.com/165298/dal-canopy-design-digitalarchitectural-lab/.

68. Pablo Esteban Zamorano, Nacho Martí, and Jacob Bek, "Expandable Surface Pavilion," ArchDaily, November 24, 2011, http://www.archdaily. com/186069/expandable-surface-pavilion-pablo-esteban-zamorano/.

69. Emmi Keskisarja, Pekka Tynkkynen, and LEAD, "Dragon Skin Pavilion," ArchDaily, March 10, 2012, http://www.archdaily.com/215249/dragon-skinpavilion-emmi-keskisarja-pekka-tynkkynen-lead/.

70. Asterios Agkathidis et al., Digital Manufacturing in Design and Architecture (Amsterdam: BIS Publishers, 2010).

71. City Form Lab, “SUTD Library Pavilion," ArchDaily, June 17, 2013, http:// www.archdaily.com/387696/sutd-library-pavilion-city-form-lab/.

72. University of Stuttgart, Faculty of Architecture and Urban Planning, "ICD/ ITKE Research Pavilion," ArchDaily, March 6, 2013, http://www.archdaily. com/340374/icditke-research-pavilion-university-of-stuttgart-faculty-ofarchitecture-and-urban-planning/.

73. Wing Yi Hui and Lap Ming Wong, "Wood Pavilion," ArchDaily, July 12 2010, http://www.archdaily.com/68446/wood-pavilion-wing-yi-hui-lapming-wong/.

74. Studio Gang Architects, "Lincoln Park Zoo South Pond," ArchDaily, October 22, 2010, http://www.archdaily.com/83676/lincoln-park-zoo-southpond-studio-gang-architects/.

75. Tongji University Team, “O-STRIP Pavilion,” ArchDaily, October 6, 2011, http://www.archdaily.com/174127/o-strip-pavilion-tongji-university-team/. 
${ }_{66} \mid$ 


\section{Design Iteration $1 A$}

\section{A Wilderness Design Dialogue}

I will now describe Design Phase One Iteration One. After the second visit to site and with the influence of Corner's writing on connection with wilderness I began to question how the site was to be occupied.

Three key phrases emerged from reading on the topic; these related to Siting, view and the relationship to detachment and attachment to the landscape,

The demographics who currently occupy wilderness sites, and

How the landscape is traditionally occupied through a building.

The following four quotes were explored further through design, site analysis, photography and physical modelling:

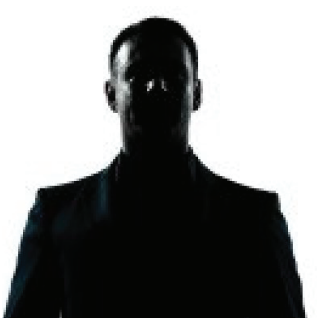

Corner (1999) reinforces the notion of the pictorial landscape; emphasising "the need for a more immersive sense of landscape \{to] critique the pictorial impulse found in nature reserves and national parks. The production and repetition of vantage points objectifies landscape while detaching the viewer".

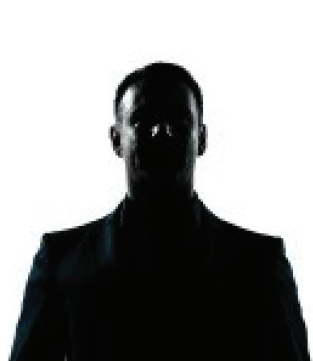

According to John Beardsley (2000);

"In his analysis he notes three classes of naturebased mindscape experience that are emerging. In the first "the affluent will make their eco-tours to the remaining fragments of pristine habitat: the middle classes will visit simulations; everyone else will inhabit marginal landscapes, salvaging and recycling to survive."'?

Jill Franz (2005) explores "landscape as a perceptual phenomenon". "Associated with this is the notion of landscape as an image. In other words, what is understood is not environmental actuality but rather a representation and an enduring image and experience of landscapeness. In the built environment, windows reinforce this extension beyond representation in various ways, the most persuasive through the mechanism of framing.

Kress and van Meeuwen (1996) in their exploration of the two dimensional image identify the frame as one of the aspects of interactive meaning, in their case referring specifically to the size of the frame and its relation to the human body. While the distance of elements in the landscape from a building is determined by a variety of factors, the size of the window, its shape, position and articulation reinforce physical and social distancing. As some designers appreciate, large expanses of glass do not put us more directly in touch with our surroundings, rather than alienate us from them."'

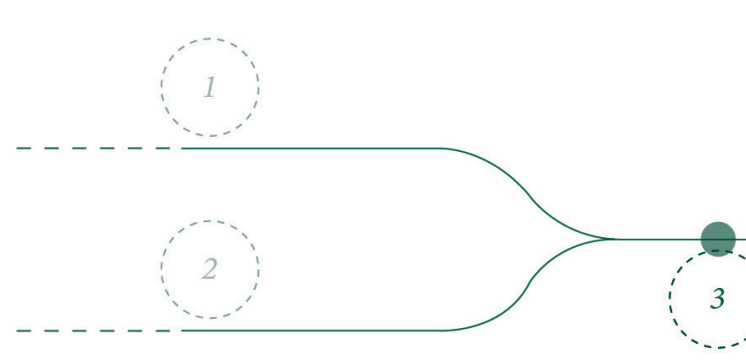




\section{Mapping Perspectives}

Corner's quote regarding view was taken and explored through the photographs of site, framing and perspective $(1,3.2)$. The three levels corresponded to:

- View, long distance shots to experiment with the concept of a viewing site and to explore whether this detached the viewer from the scene or formed an attachment.

- Sites that combine a close foreground element and a view, to explore if this will connect as opposed to detach the viewer while providing an attachment forming view.

- Pure foreground elements, with little to no porosity so there is no experience of a wider area.

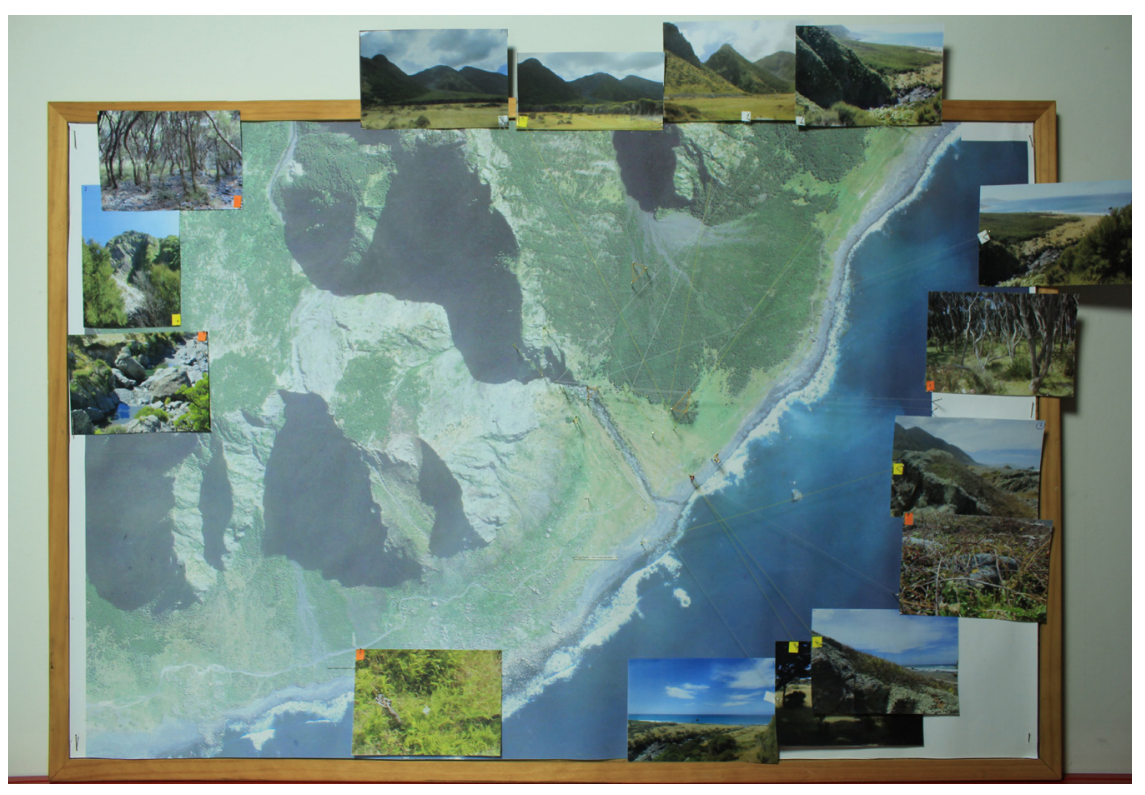

Figure 3.1

Analysis of survey responses and translation into a series of model tests which explore the category "no evidence of impact".
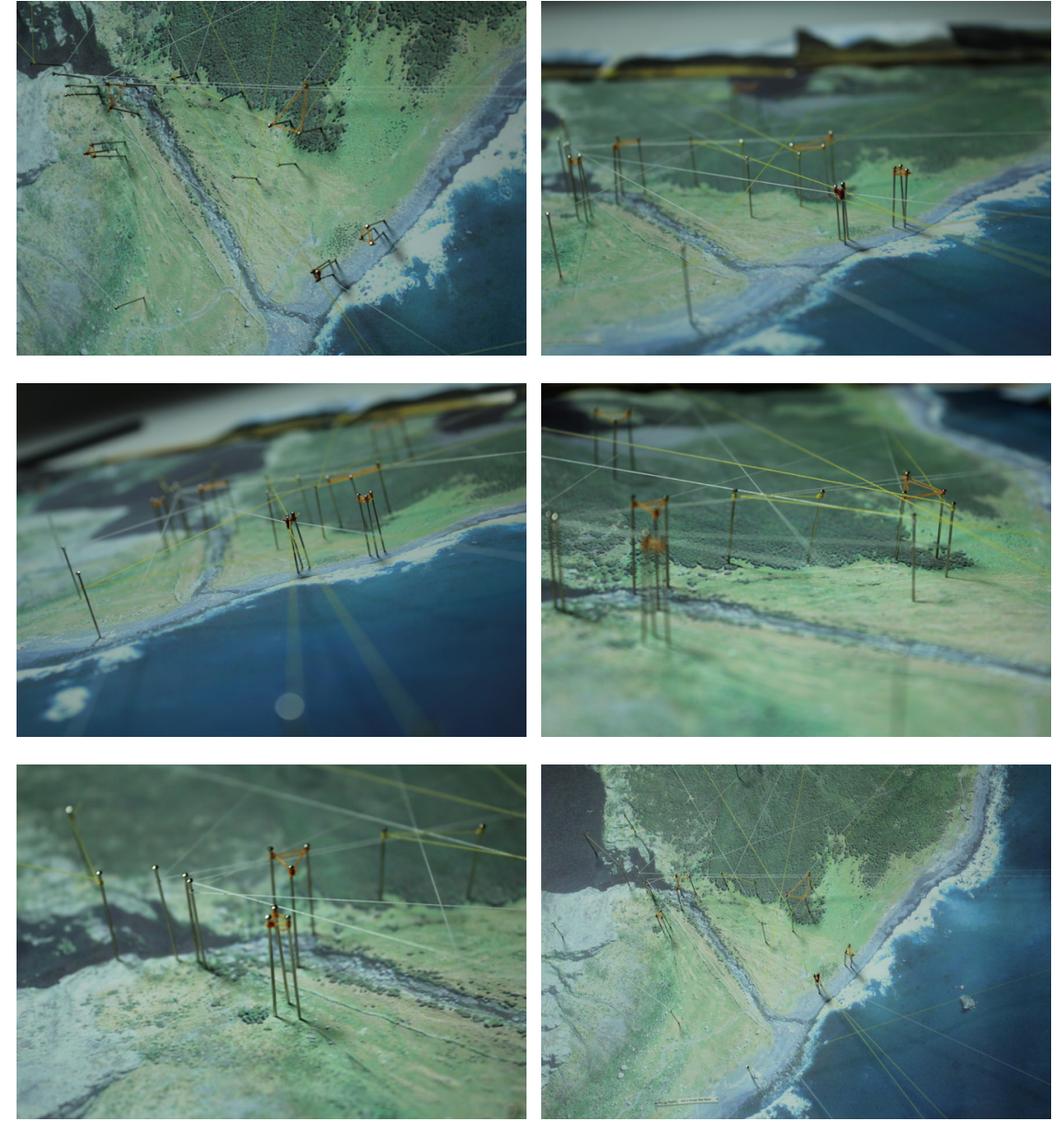

Figure 3.2

Analysis of survey responses and translation into a series of model tests which explore the category "no evidence of impact". 

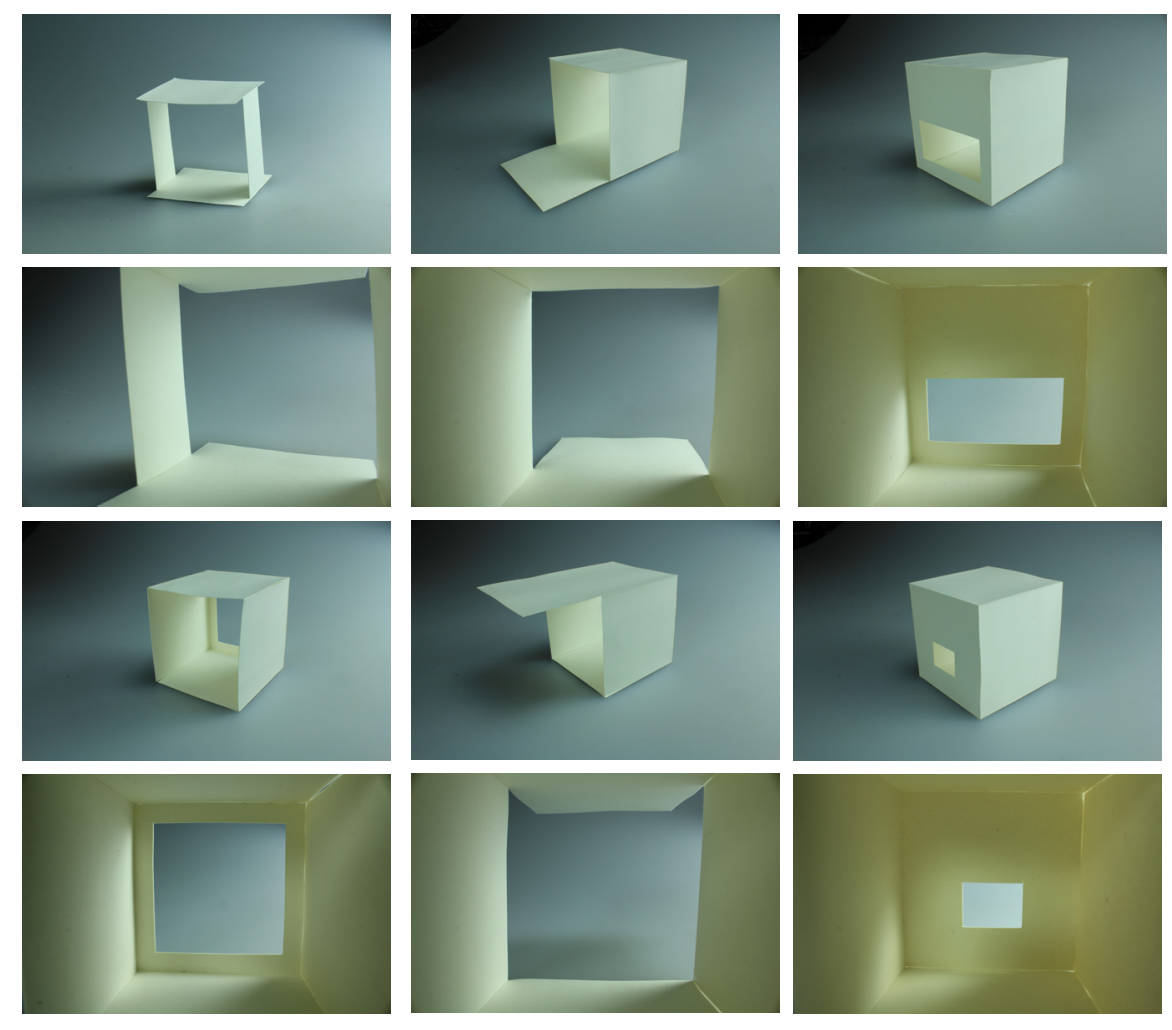

Framing

John Beardsley's quote referring to the framing of a window and detachment versus attachment was then explored through small models in relation to the same views of site (refer to 3)

Each of these simple forms is designed to explore the framing of the landscape and where the architecture is the viewing mechanism. This relates more directly to occupation of space and although these are simple and basic forms, this exploration tests the conventional 'experience' of architectural framing.

The models begin with the least obstructed and open forms and gradually become more enclosed until just a small select glimpse of the exterior is visible.

\section{Figure 3.3}

Physical models for the framing exercise 


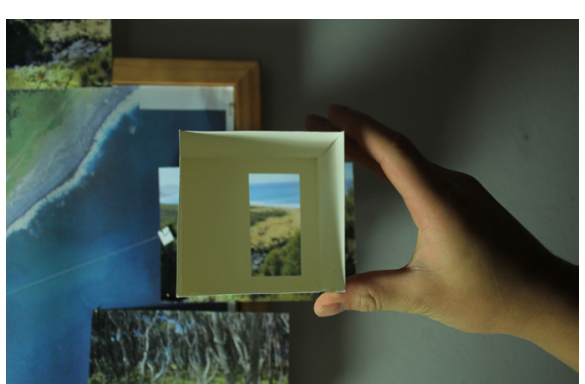

Process
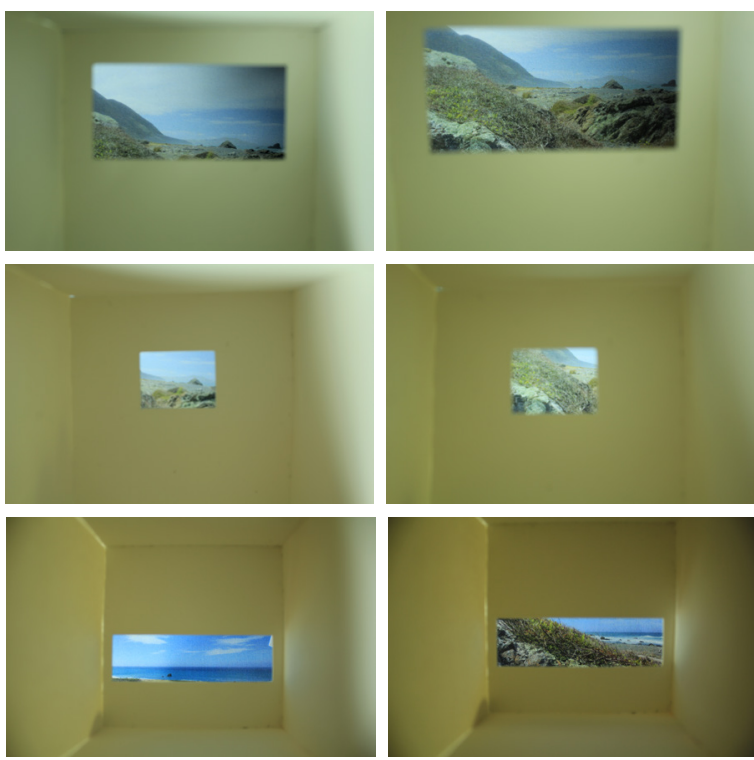

1. Sole Background

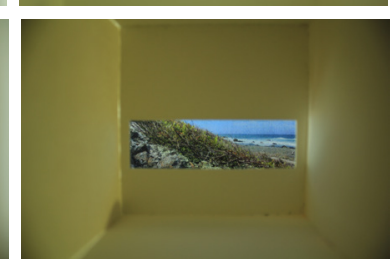

2. Foreground + Background
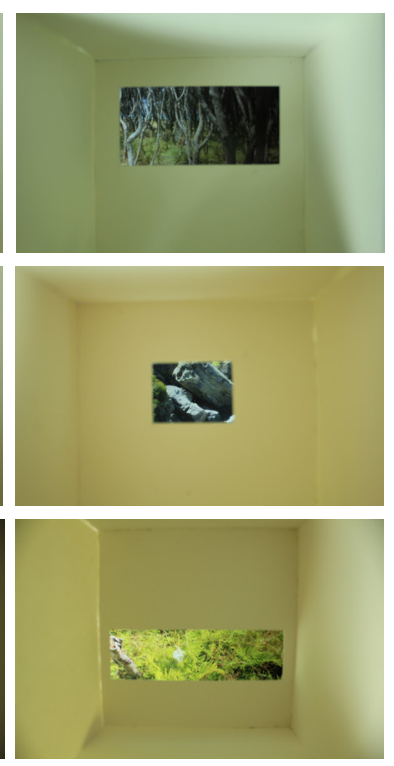

3. Sole Foreground

\section{Perspective Interaction}

Each of these images is designed to frame the view differently to gain the sense of boundaries, interaction, detachment and attachment.

My personal findings are that the large expansive view has the most impact but detaches the viewer from their direct surroundings, reinforcing Corner's (1999) statement. The middle view that has a layered combination of both foreground and background is the most successful in maintaining impact while keeping the occupant directly aware and involved in their surroundings (refer to Figure 3.4). The third option of a purely foreground dominated scene is successful for interaction with the direct environment, but is limited in maintaining interest with view. It has to be noted that these experiments are limited to the visual and the other senses would alter the connection. In retrospect a figure to give these images scale would also help understanding of space.

\section{Figure 3.4}

A series of investigative tests were carried out which explore framing content; sole background, foreground and background, and sole foreground, to see how it influences attachment and detachment to site. 


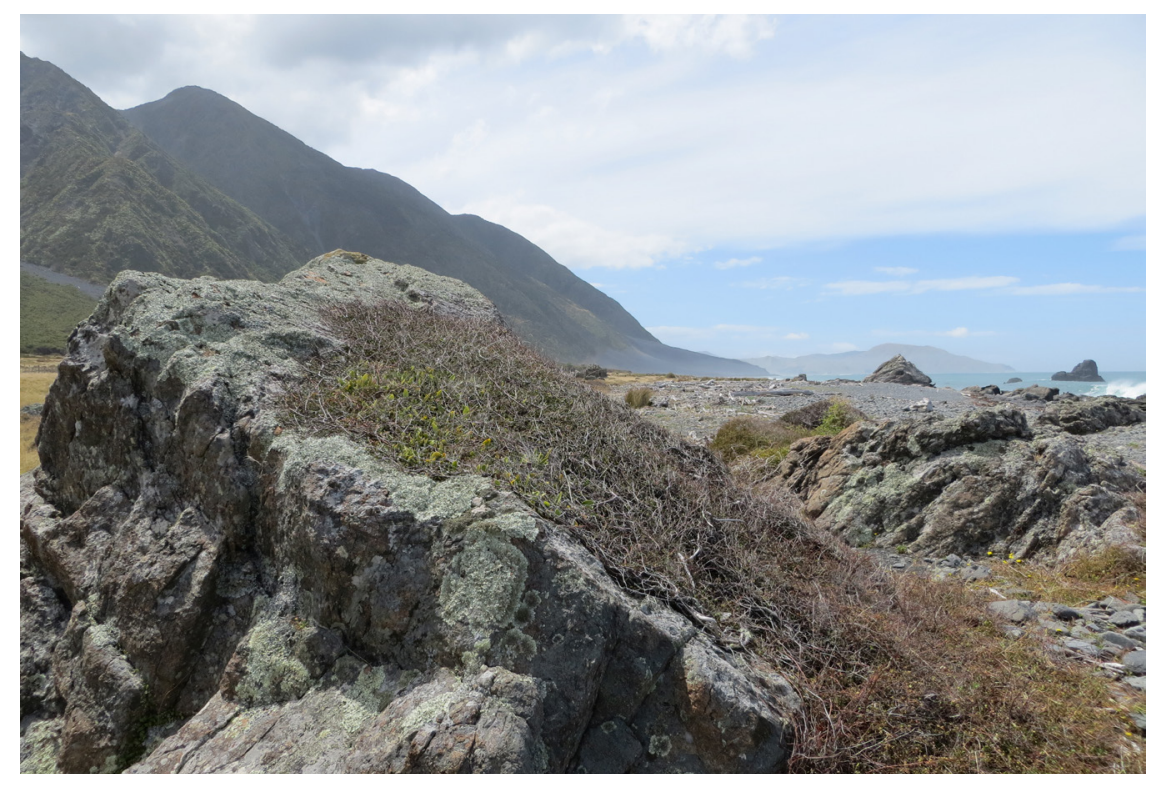

Figure 3.5

This image displays an example of a successful site with foreground and background elements. The overlay translates the perspective from the photo and define the range of the view to give these foreground and background elements

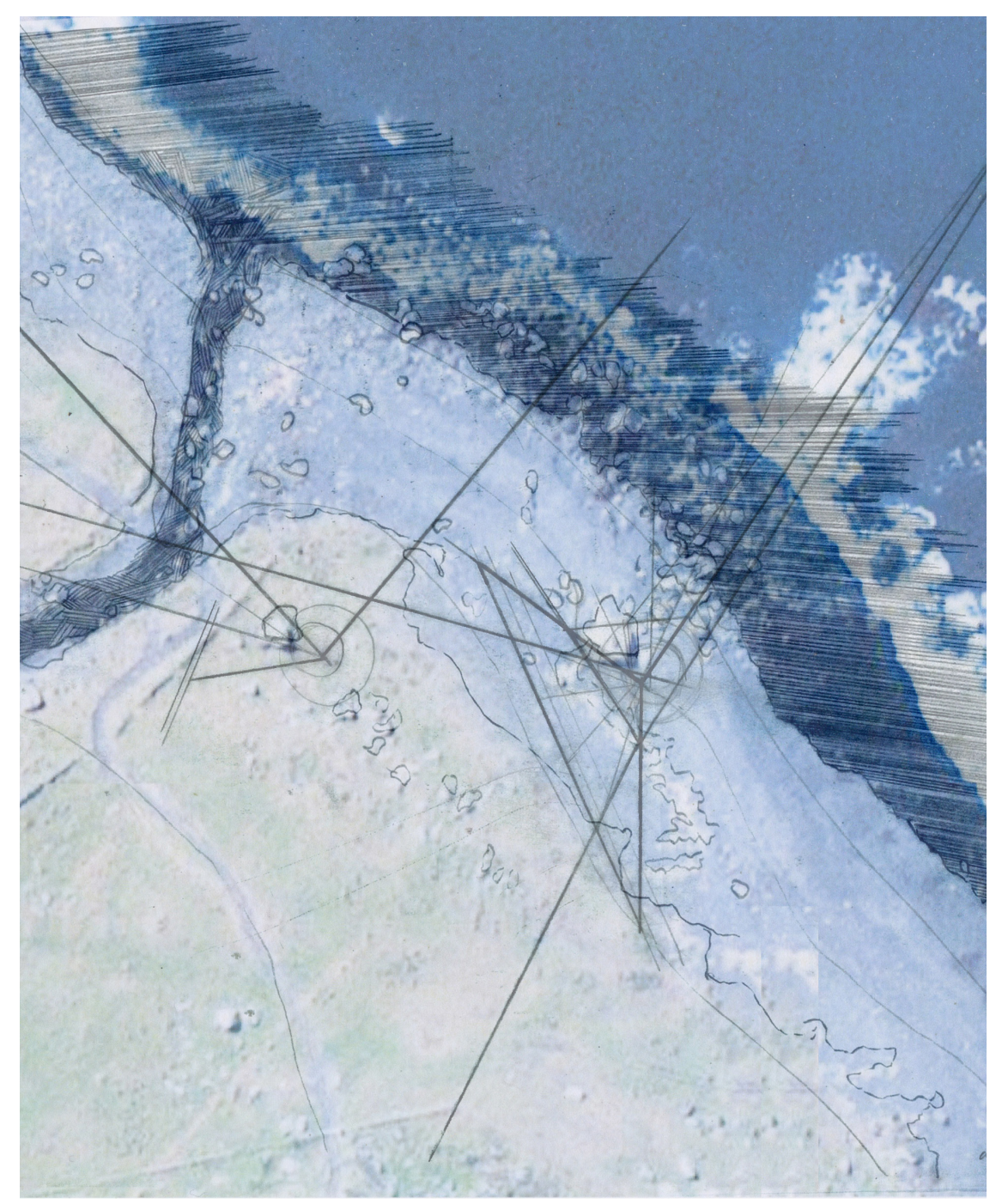




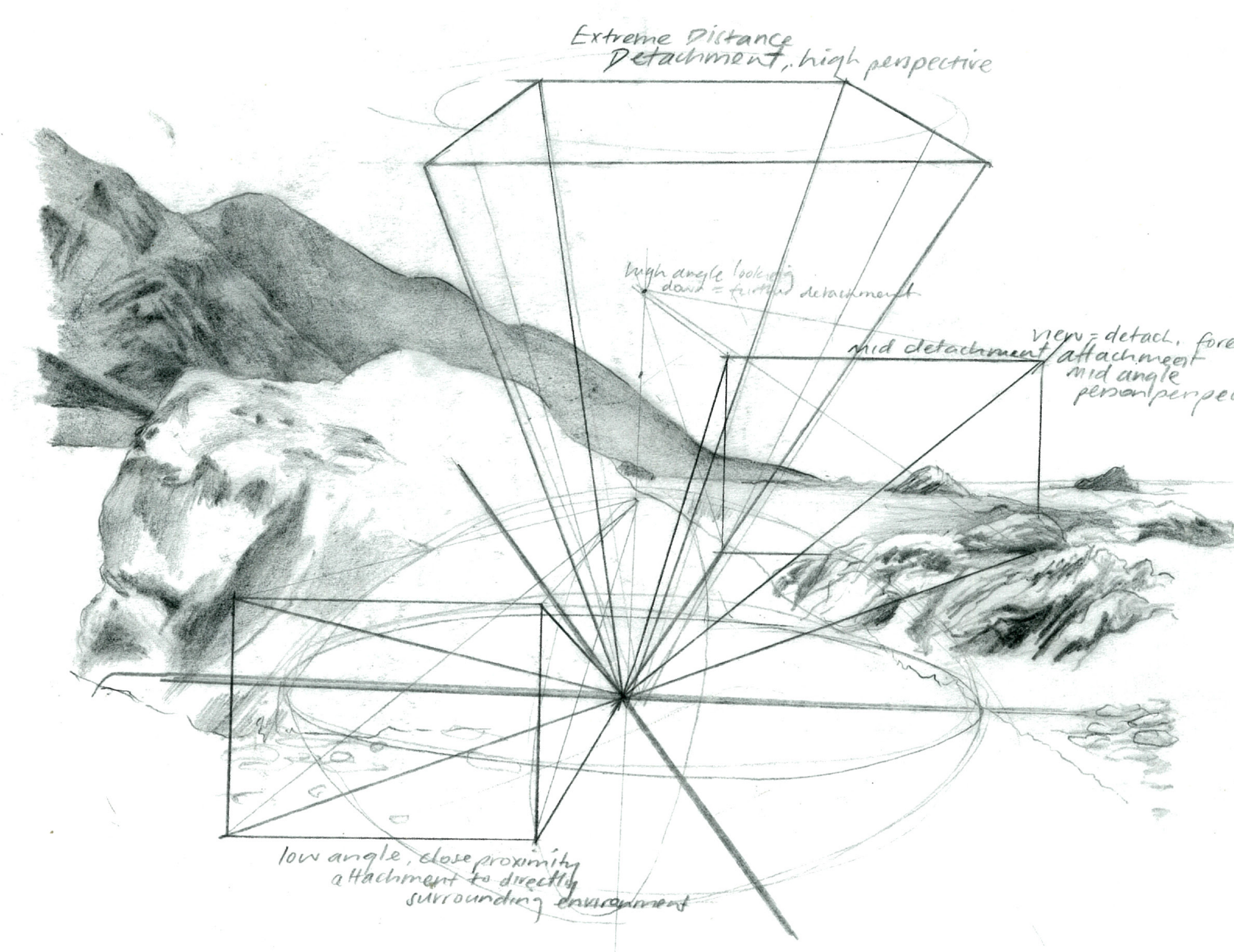


This investigation revealed that aesthetic attachment was formed through the combination of dominant foreground and background elements in the view plane. The large foreground scale also extended this purely aesthetic exercise by exploring the theoretical relationship between visual and haptic. ${ }^{4}$

In response to the DoC tramping hut precedent analysis I started by constructing a series of models that analyse the relationship between interior and exterior, wilderness and architecture. I again returned to Corner's comments on the current aesthetic experience of landscape. ${ }^{5}$ I began to examine the idea of inverting preconception, by bringing the wilderness into the architecture, and taking the architecture out to the wilderness. This touches on ideas of attachment and detachment (also referred to in the 'non-static' section) and of a sense of user ownership over the landscape.

In 2001 the geographer John Shultis surveyed the current understandings of 'wilderness' across a representative group of New Zealanders. ${ }^{6}$ The results from this survey formed ten categories, one of which was "no evidence of impact". Initially this category was developed through a series of design tests decomposing the New Zealand DoC backcountry tramping hut to a form that has a similar visual language to its surroundings. (refer to Figure 3.7- 9)

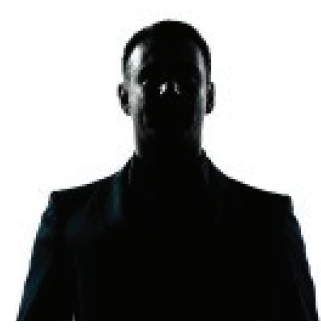

[John Shultis, through the survey, discovered that a] high rate of people able to define wilderness even though they had never experienced a wilderness region as defined by the Department of Conservation. ${ }^{8}$ From the responses [to his survey] Shultis formed ten cumulative categories which in order of preference were:
1. "Bush/native forest
2. No evidence of impact
3. Trees/forest/vegetation
4. Peace/solitude/freedom
5. Remote/isolated
6. Primeval/original condition
7. Nature/scenery/beauty
8. Mountains/alpine
9. Animals/birds/wildlife
10. Rivers/waterfalls" 


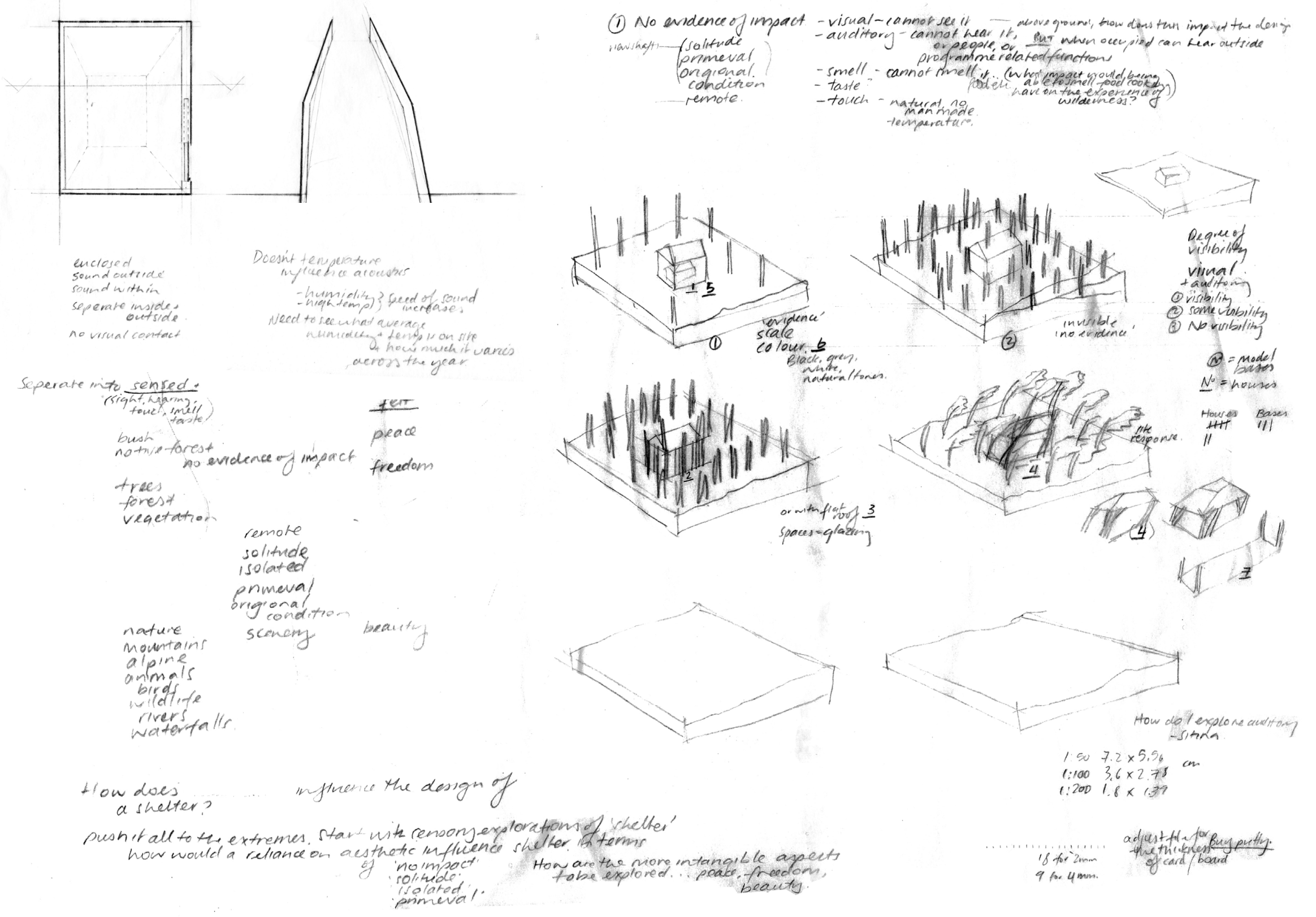

Figure 3.7

Analysis of survey responses and translation into a series of model tests which explore the category "no evidence of impact". 


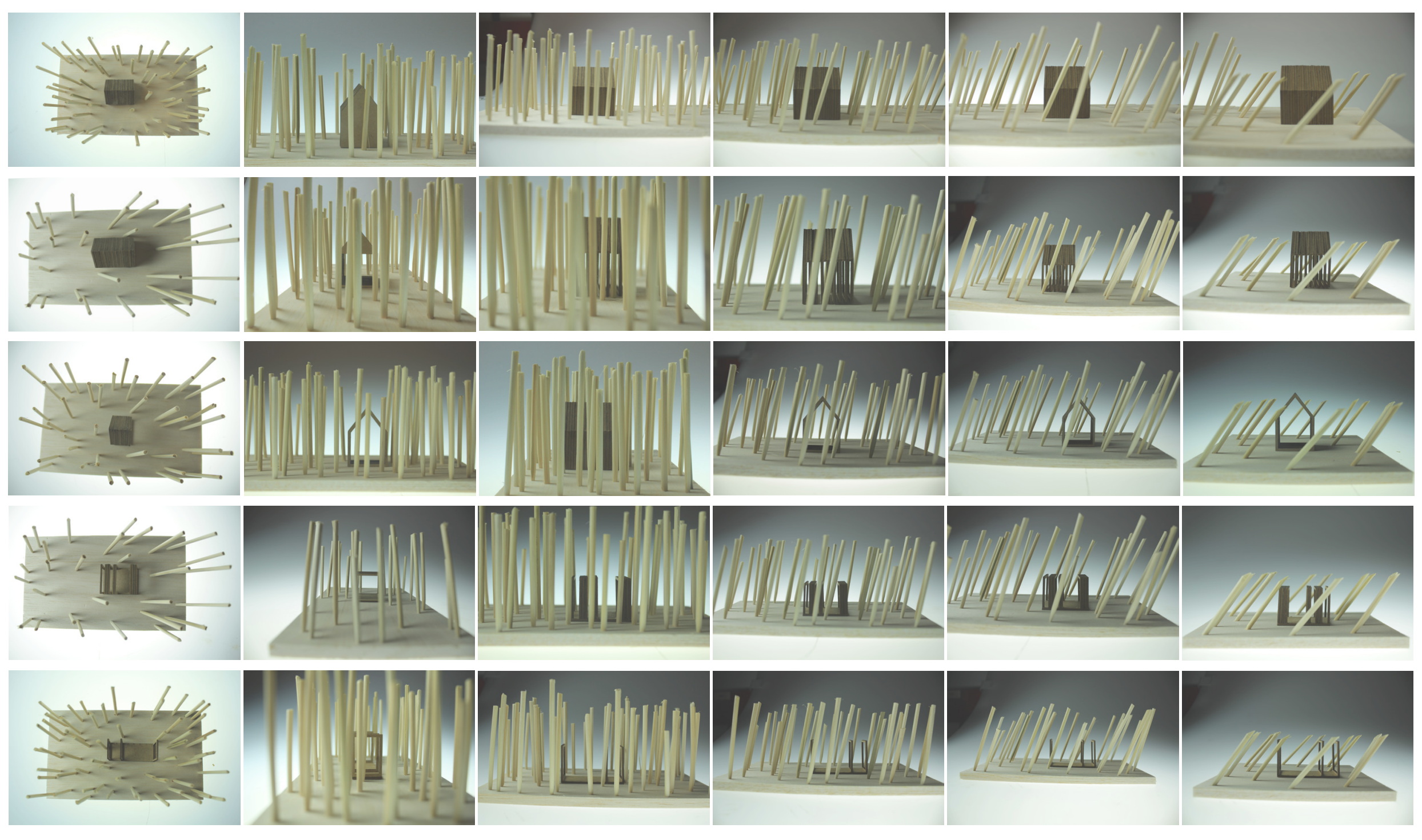

Figure 3.8

A modelling study into visual mass and form relative to the landscape.

\section{Method:}

I began by generating 'landscapes' derived from the Ngapotiki Fan site. They range from the taller upright trees to low and less dense bush the further you ascend the scree slope. I then designed from the basic form of the New Zealand backcountry hut as one solid mass, and started to reduce the visual density by creating openings and changing massing.
I continued this and moved to square forms, as well as some alternative abstracted forms, and forms that responded to the dominant shapes in the landscape such as tree trunks. Each model was placed in the four landscapes and analysed through photography. 


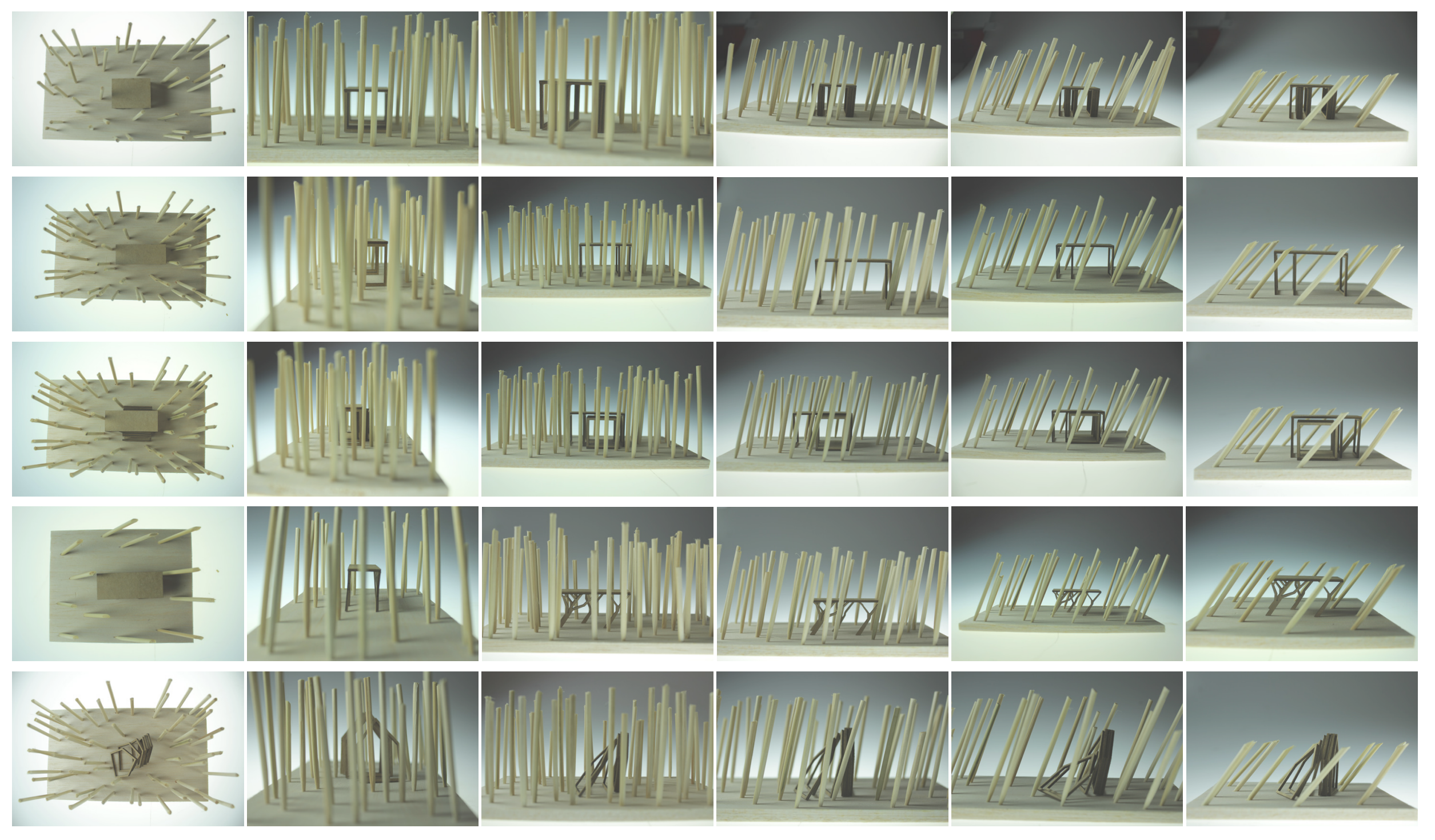

Figure 3.9

A modelling study into visual mass and form relative to the landscape.

\section{Outcomes:}

These tests begin to explore a form scale from the backcountry hut, with its tried and true form for weather and space optimization, to more abstract forms that near the sculptural and potentially uninhabitable space. The rectilinear box forms again show a strong coherency with the vertical forests but do not relate well to the windswept landscapes, instead they seem juxtaposed in the landscape which highlights effect of the wind on the natural elements. I think this investigation was too limited by simple hut forms but this same test can be tried on future designs of shelters to analyse the visual impact. 


\section{Informs Research:}

This massing study informs my research by engaging with the idea that wilderness can be physically intertwined. For example, if the sticks in the models were not representative of trees but were poles that were added to the dense forest landscape periodically around the architectural design, to further diffuse the boundary between natural and unnatural. A decentralised group of spaces are created with a designed engagement level with the wilderness.

Using the modelled forms that were more successful in relating to the tree cover on site a design concept was developed through drawing (refer to Figure 3.11). The building begins as a closed compact shelter of four modules with no entry and slowly slides outwards until the occupant can enter it (Figure 3.13). The architecture continues to extend outwards until each module has greater than ten metres separation. The distance between buildings is great enough so that the wilderness within the architecture is still a small enough region for the users to feel a sense of ownership over the space. The programme forces the occupant to pass between interior and exterior, wilderness and architecture to carry out a conventional routine. The glazing of each unit faces inwards to ensure that every view frames architecture and wilderness.

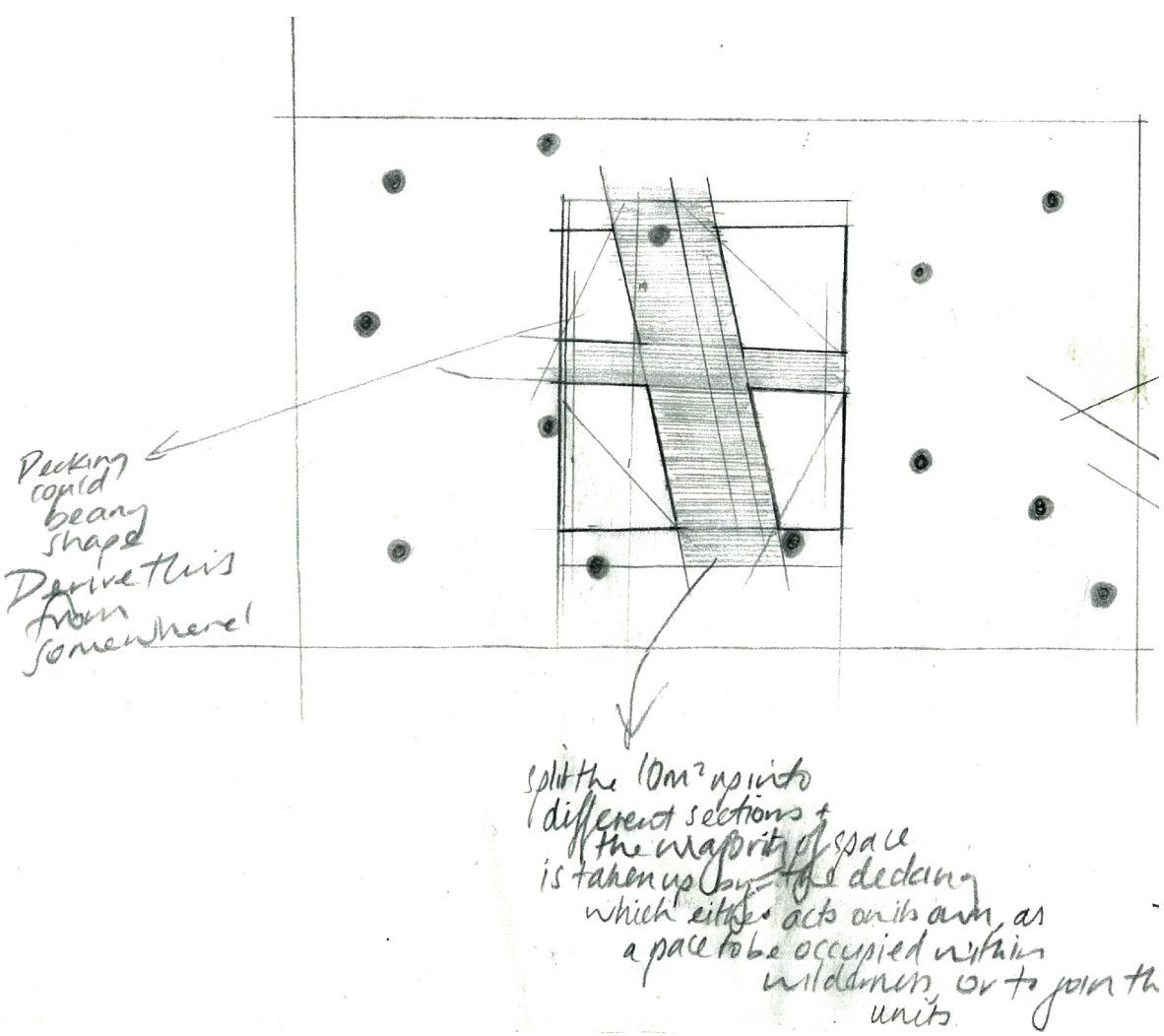

Figure 3.10

Sketch plan showing the initial idea for external transition spaces and internal programmed rooms within the forest-scape. 


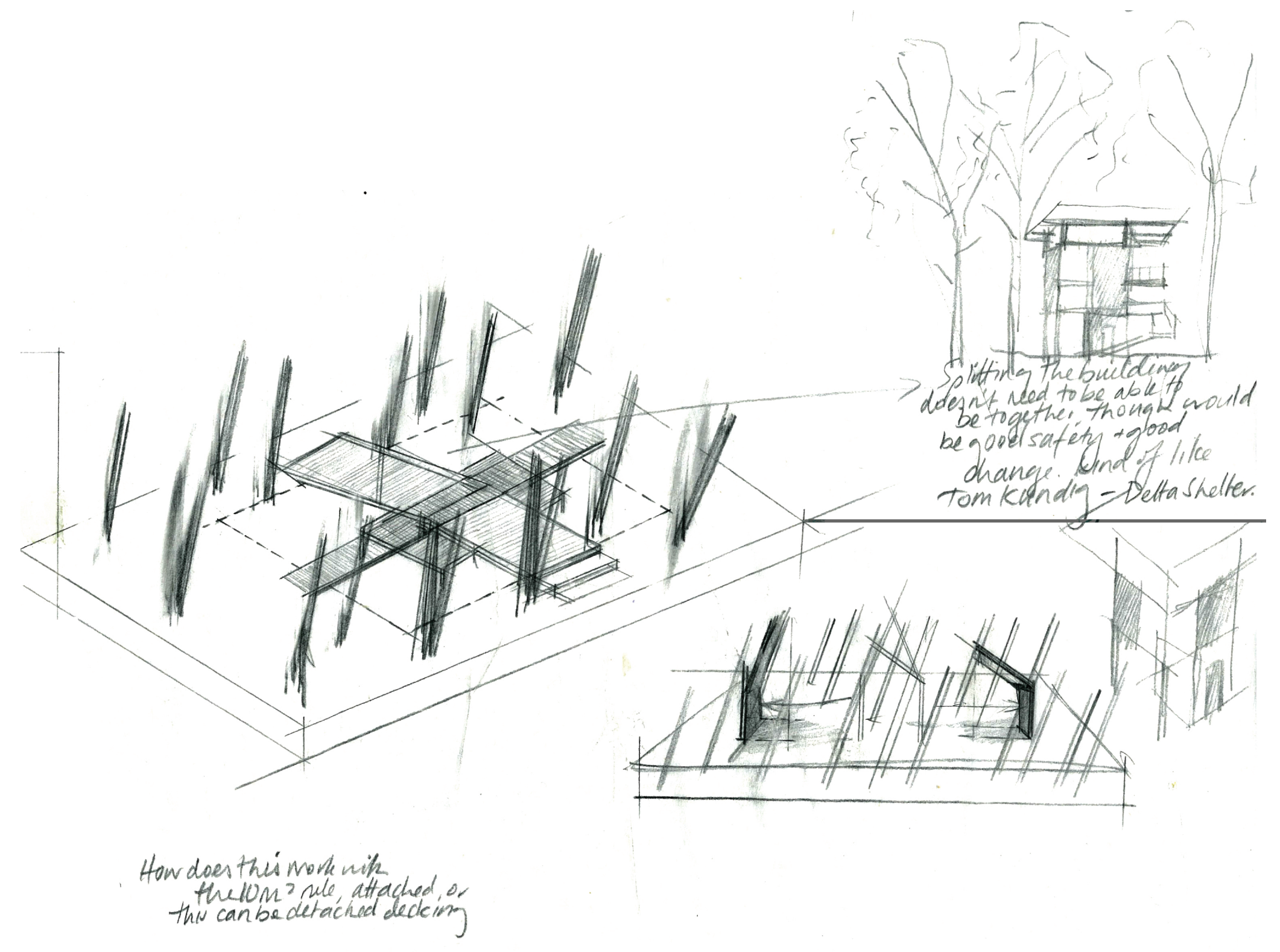

Figure 3.11

In this sketch concept programmed rooms begin to be decomposed and reduced to minimum basic structure, shelter and functional requirements such as cooking facilities, bathroom fixtures.
Kundig's Delta Shelter is able to be closed off when not in used for security needs. This raised a critical issue for a divergent design as there is a large amount of foot traffic along this coast. The following concepts take this into consideration. 

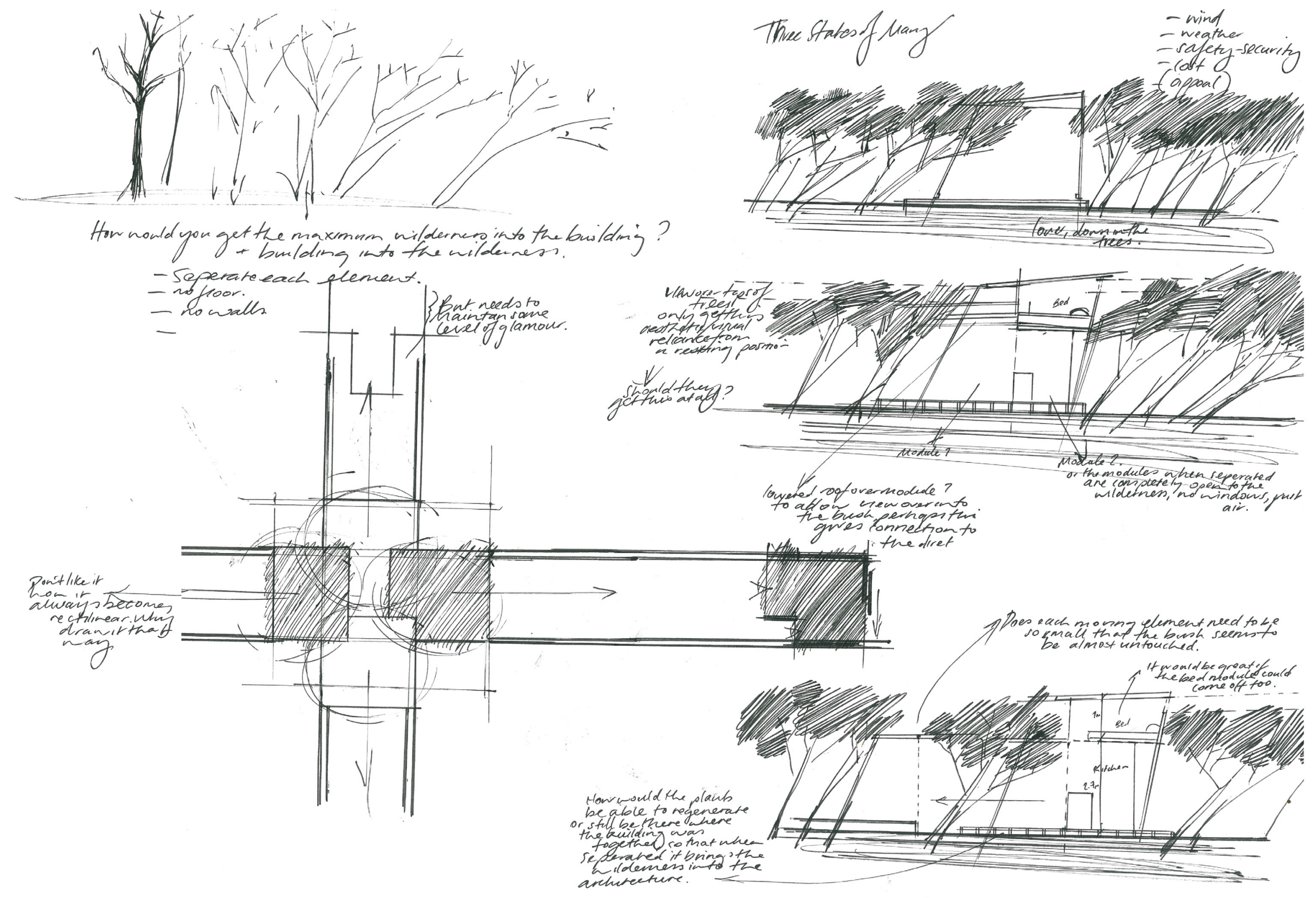

Figure 3.12

The idea of moving elements is again introduced, this time as a security precaution. The enclosed rooms move outwards from the centre to create the transition wilderness space in between. 


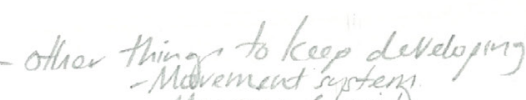
- decide ixposedyinindars

wildemen Houses Exeras creating whetectire fropt the massing
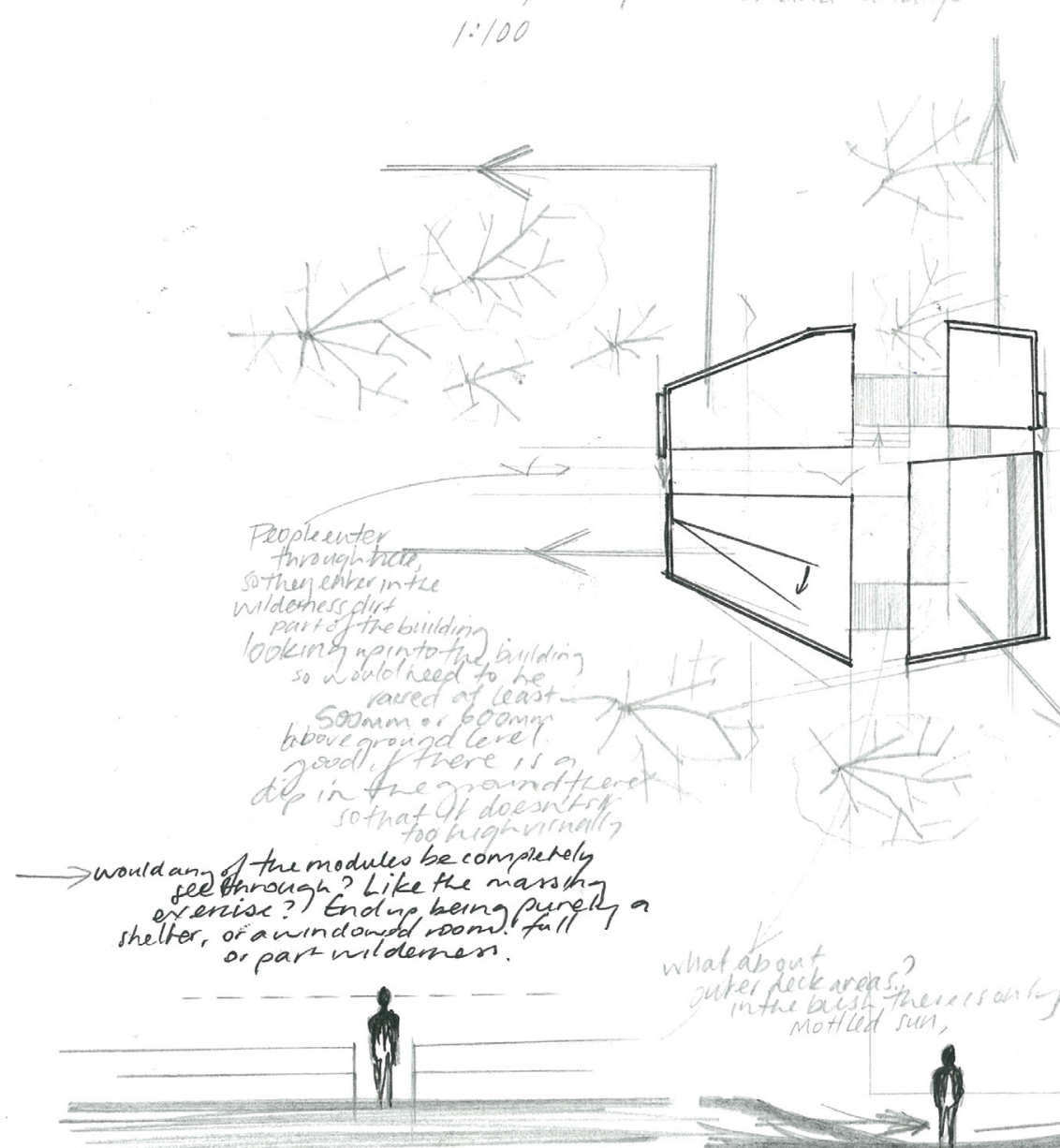

sel through? Like the massth exerise? Endup being punger a

or part wildernes.

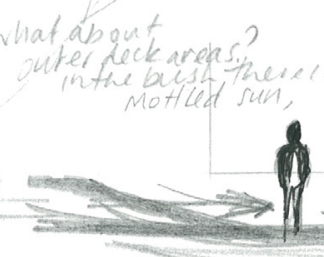

This option would need glazing?

ir pernapsnot, ust methoch of

Figure 3.13

More developed concept sketches explore how a person enters the building and how to maximise contact with the ground plane before entering the rooms.
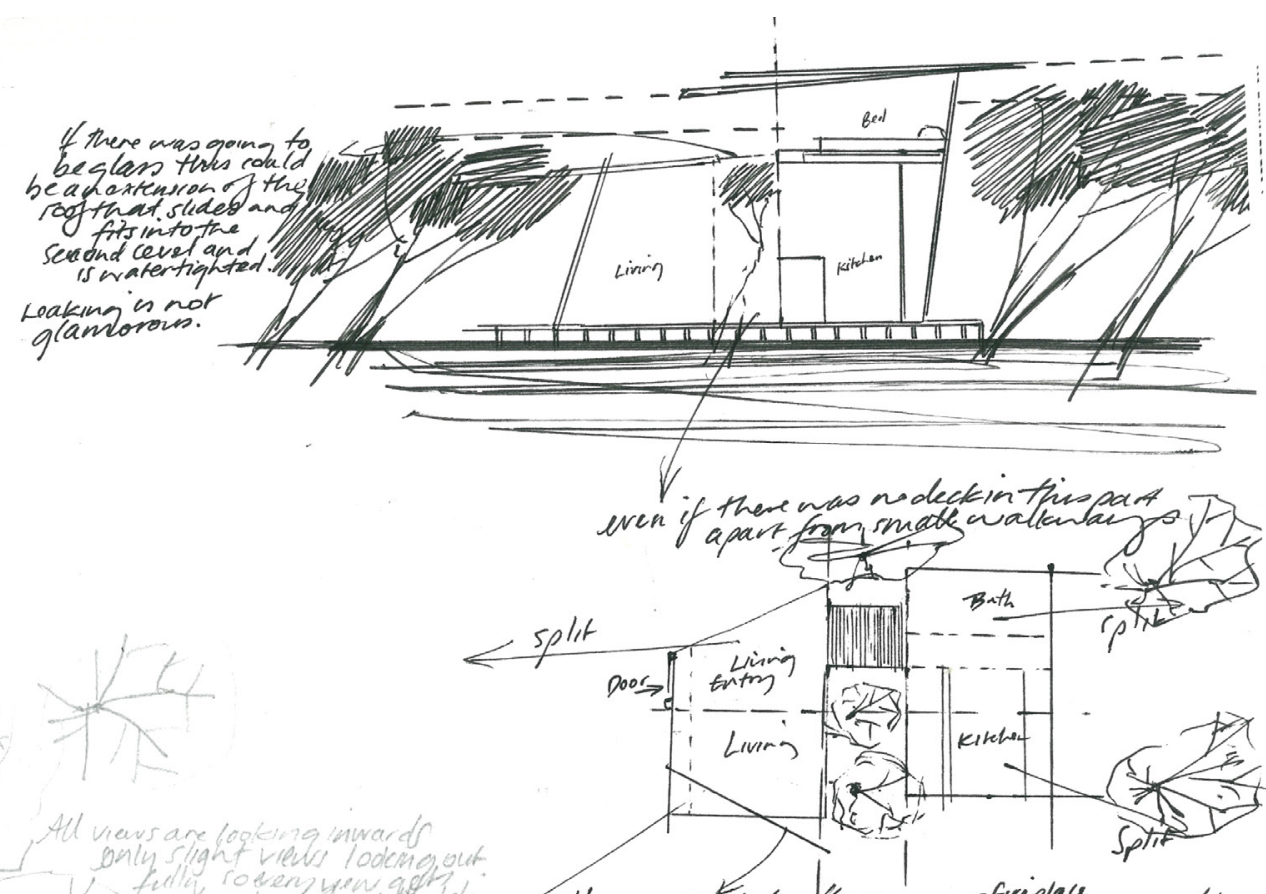

atoced wates

- Fireplace

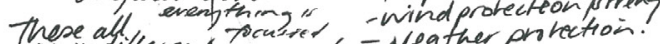
split in different muan. Saming and forped/movement

deas, Lind can move thote poles in the these cround to begint ho:

change the location inducare the artas some wind provection in the
trees and shiandos do. also like kundig

- wheels

- matother.

- rollers like arroort luggage set in to the ground subteny?

- Each of there could essentially be $\mathrm{OM}^{2}$, because then can be seeh as

seperare buldings srought Butne dont one

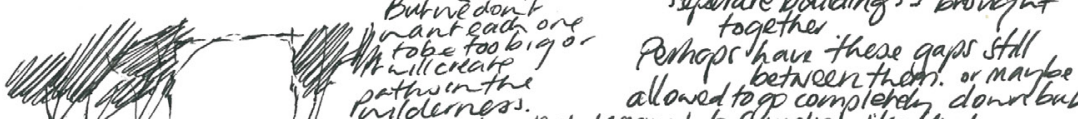
Pailderness that could

allowed to complehes. don but engage wion fuistatung archerecture
The building is raised off the ground plane so that the architecture and wilderness are emphasised through separation. 


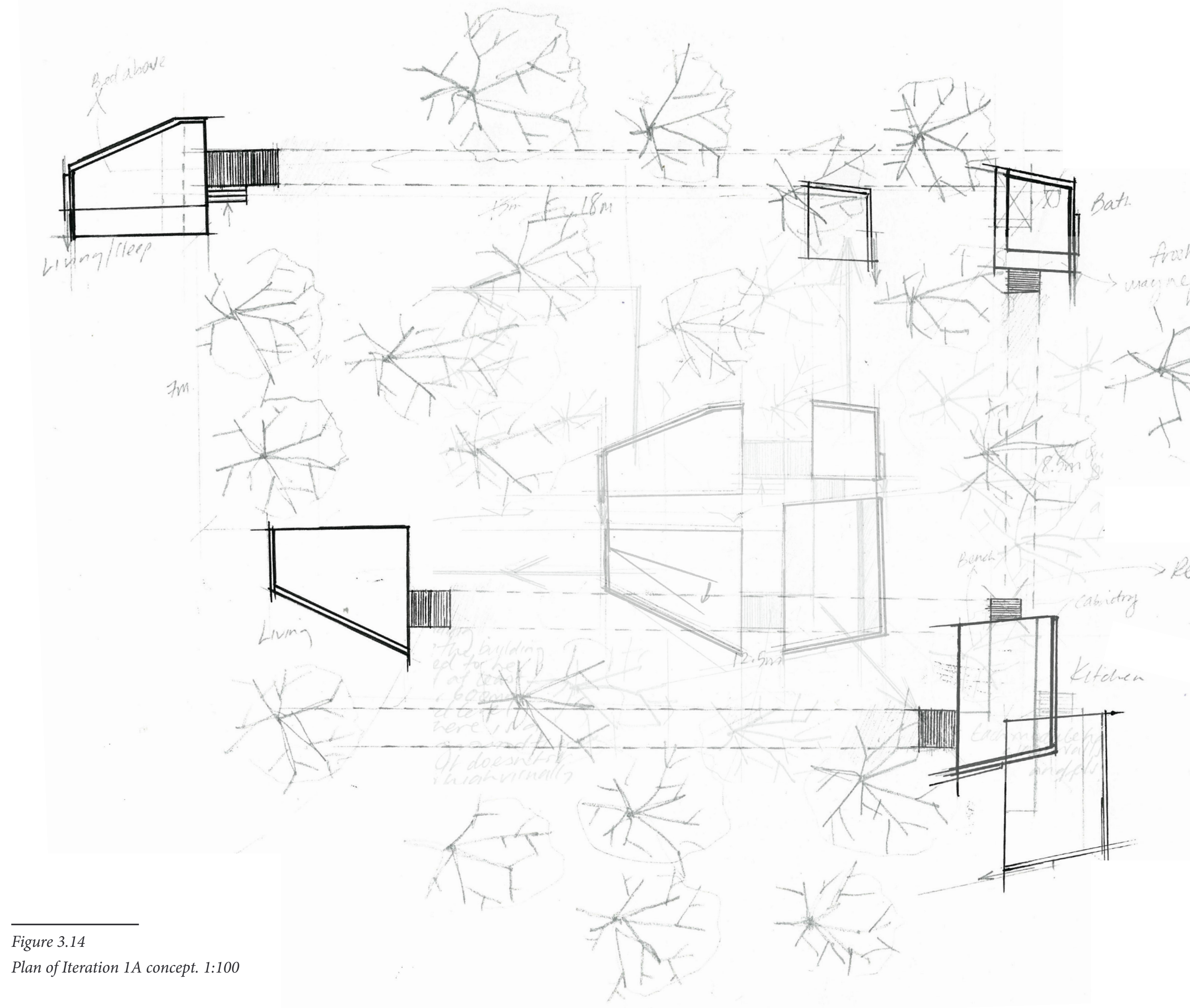




\section{Reflection}

This series of design tests followed a more archetypal approach to designing a semi-permanent shelter in comparison with the two following iterations. The final concept has the potential to embody a classic sense of glamorous space. The design prioritises comfort, detailing and conventional construction systems yet challenges the users preconceptions of space in more subtle ways. The programme necessitates passing between interior and exterior for each different function to encourage the person to interact with their surroundings. The architecture and landscape are both occupied on an equal level so the idea of a wilderness environment is no longer a rare, isolated experience.
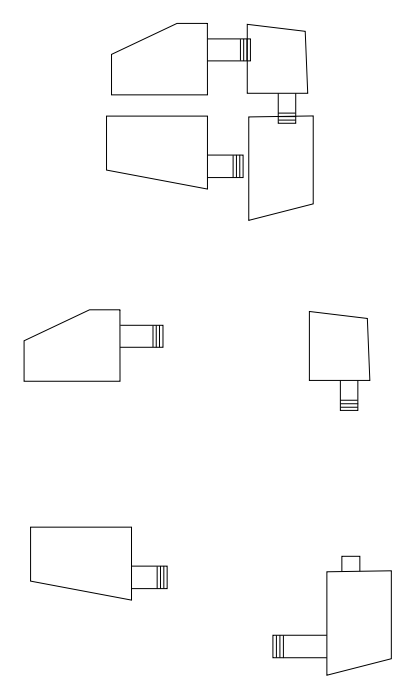

Figure 3.15

Architecture out to wilderness and wilderness into architecture diagram. 


\section{Design Iteration $1 B$}

\section{The Engineering of Affect through Design}

This design phase focussed on Shouse's definition of affect as an abstract "uncontrollable, unformed and unstructured entity. ${ }^{10}$ Site conditions, biology, ecology and geography were explored to find an element that exhibits these same characteristics. Weather, specifically wind, emerged as the site condition that is uncontrollable, unformed and unstructured. This was the motivation for the set of precedents describes and analysed in the Context, Chapter Two.

These characteristics can be applied through design to dramatise the wilderness. By involving an uncontrollable and unstructured entity in the design process, there is the potential for the design to be something more than controlled conception. Architects are limited by aesthetic and social expectation from clients, building requirement, engineering, and their own archetypal understandings. By involving an uncontrollable variable in the design process something truly new can emerge.

Initially the affect case studies were examined and were found to be similar through their dramatization and control of nature. Each case study isolated a natural phenomena (e.g. rain or bird flight), placed it in different surroundings and then dramatized that phenomena. This concept tested the idea that a building can keep its archetypal details, windows, walls, roof etc., and small moments within the shelter can be designed to draw attention to qualities of the landscape (refer to Figure 3.7, 3.8). By drawing attention through small carefully detailed experiences the occupant has a glamorous stay while recognising the beauty in the natural environment.

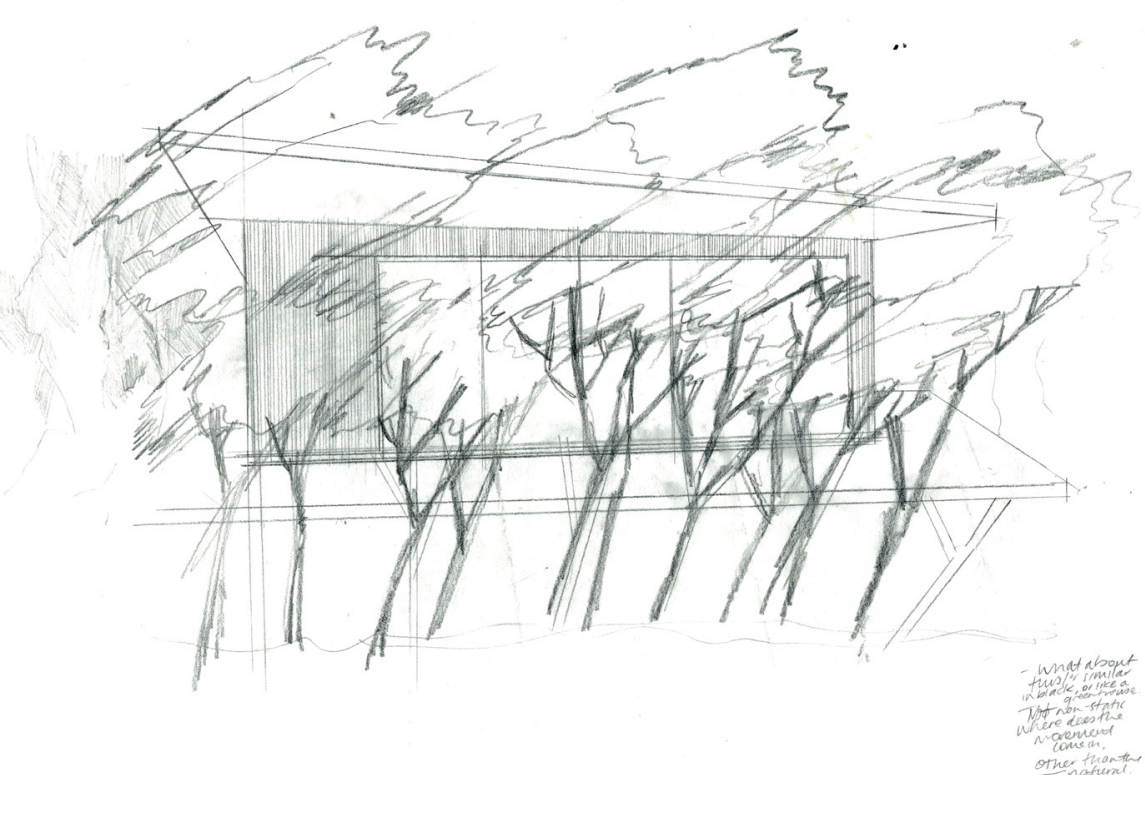

Figure 3.16

Front elevation for Iteration $1 B(1)$

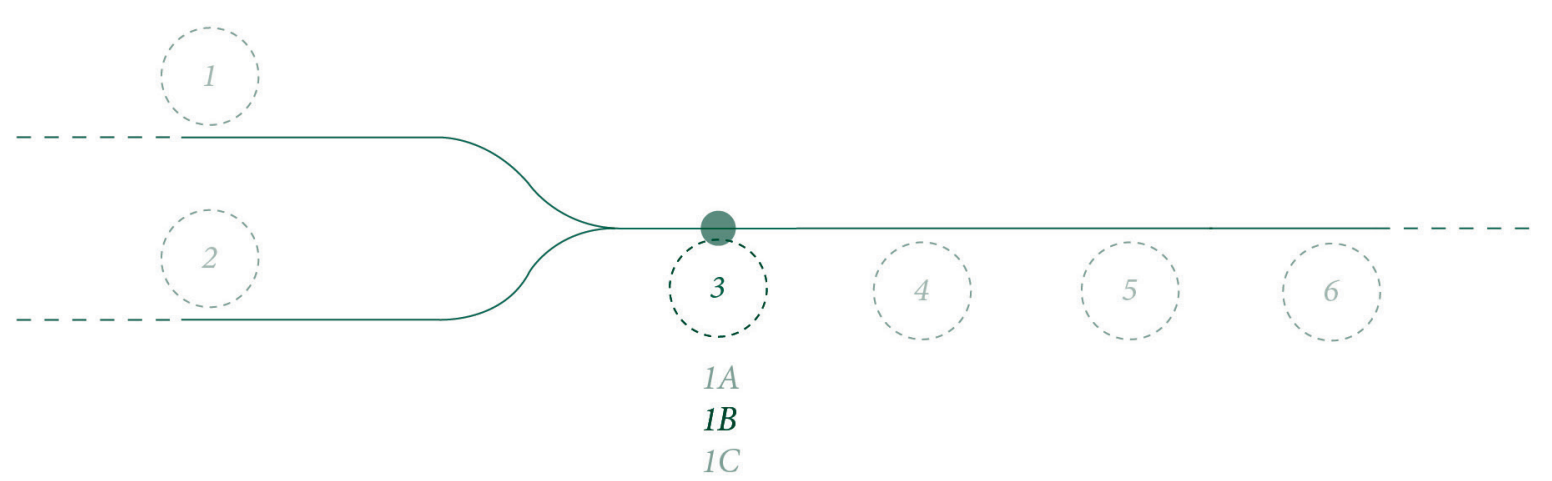




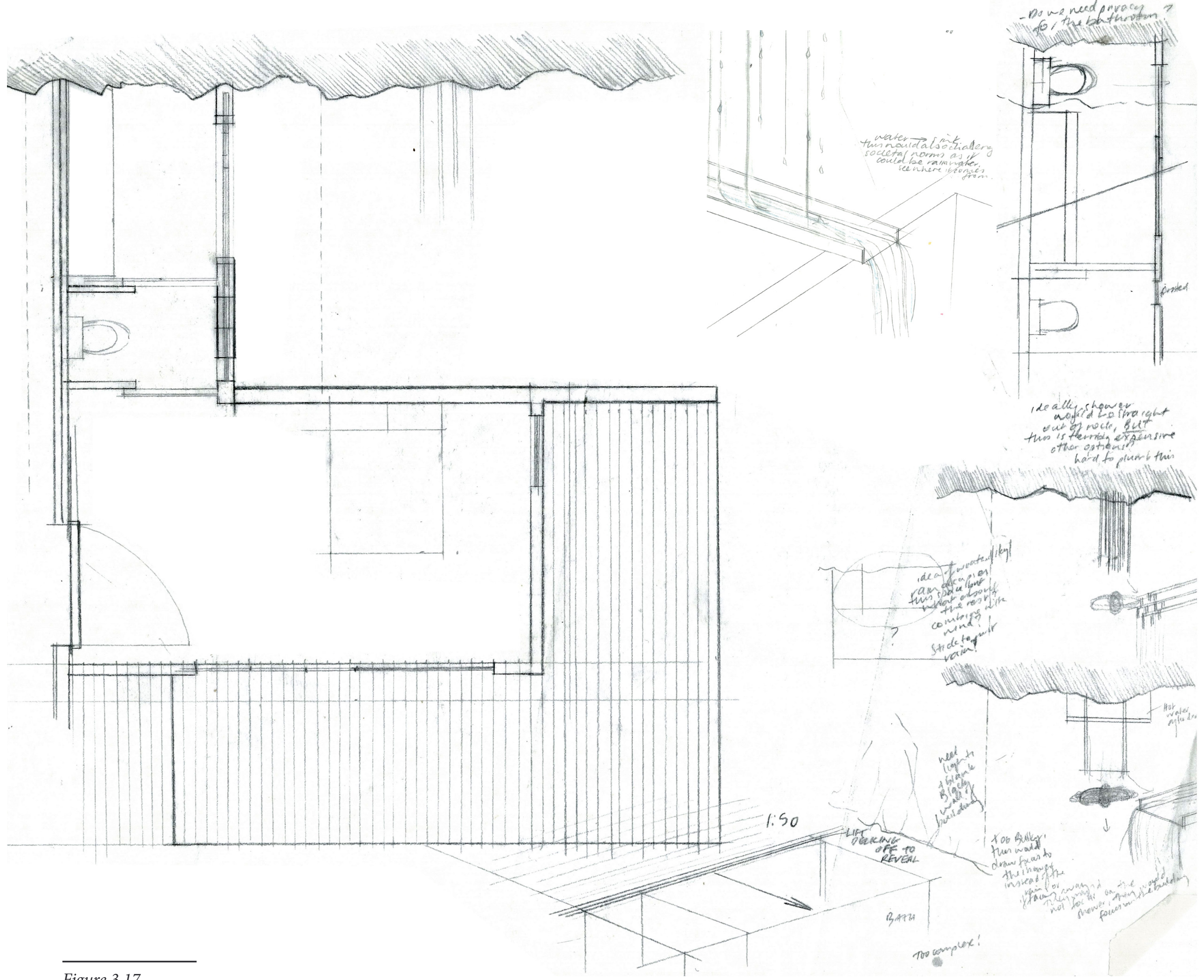

Figure 3.17

Iteration 1B(1), Floor Plan 1:50.

Small dramatising details are tested within an archetypal design.

85 


\section{As Craig Potten summarises:}

\{Wilderness is\} "A powerful antidote to the controls of civilisation, a place and state of mind where the individual's imagination [can] soar beyond its social conditioning." 11

The architect still has the role of designing in response to controls; designing parameters such as joints, structure, materials that limit the windarchitecture interaction to an isolated effect. I explored this idea through the creation of a semi-static jointed timber structure where the wind dictates form.

This concept was given a programme, modelled physically and manipulated by hand (Fig 3.18-3.21). This allowed me to see what structural controls are needed while still maintaining interactive wind movement in the shelter.
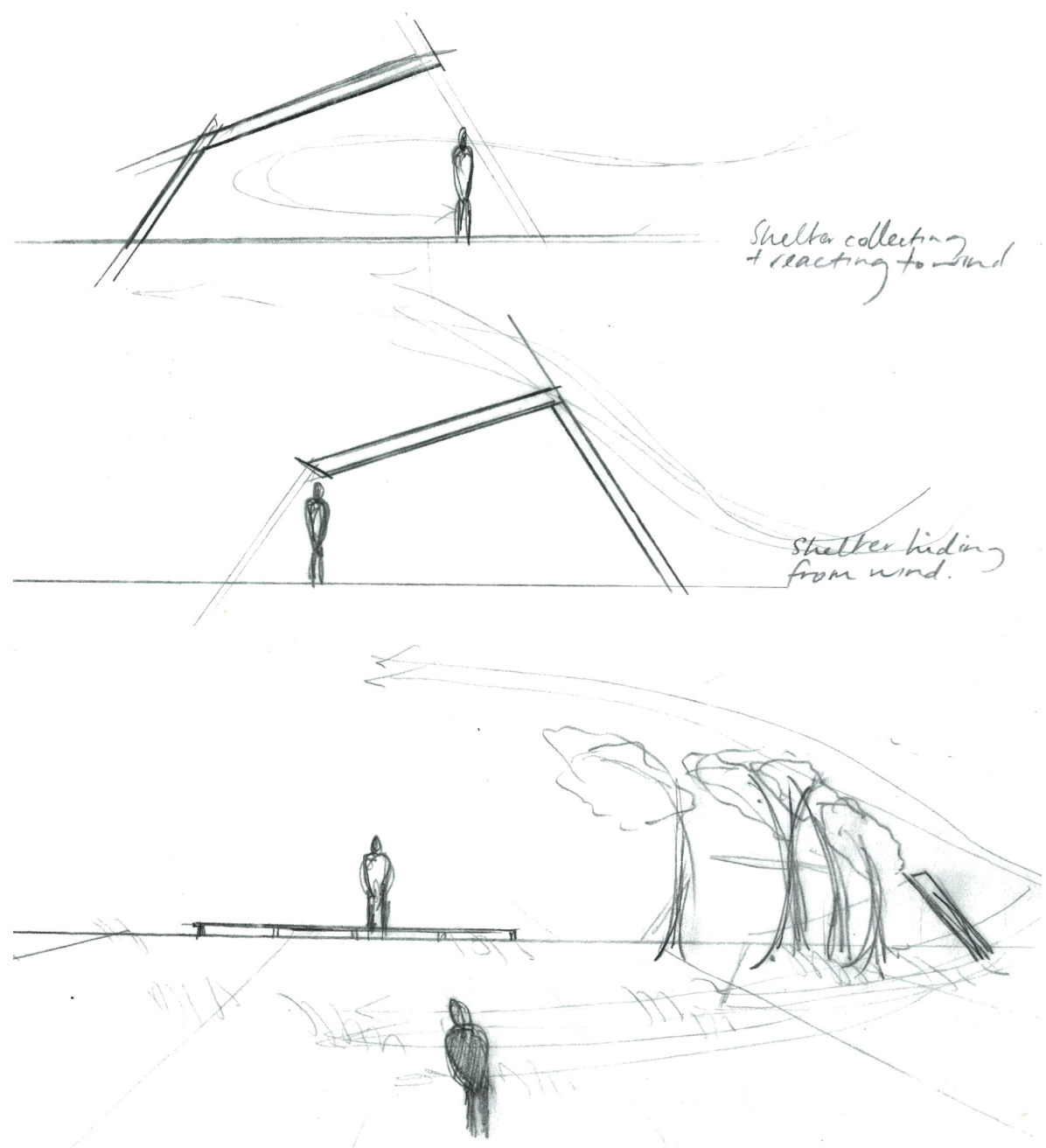

Figure 3.18

Diagrams exploring the relationship between the shelter and the wind. 



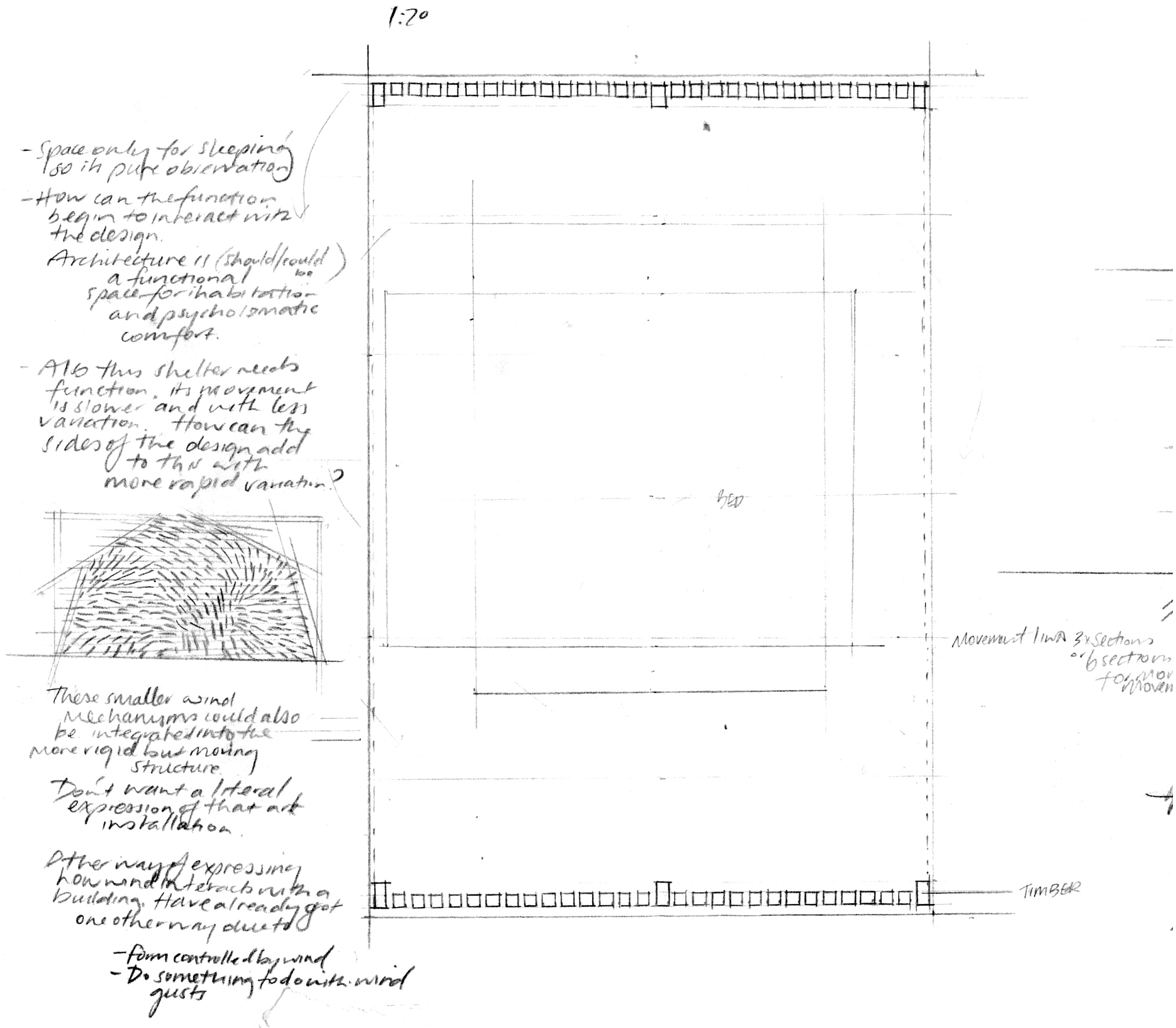

\section{Figure 3.20}

The wind alters the form of Iteration 1B(2) to express how wind interacts with the faces of a building. The joints are tight so only extreme winds can change the form of the shelter. Programme remains a simple bed and is 

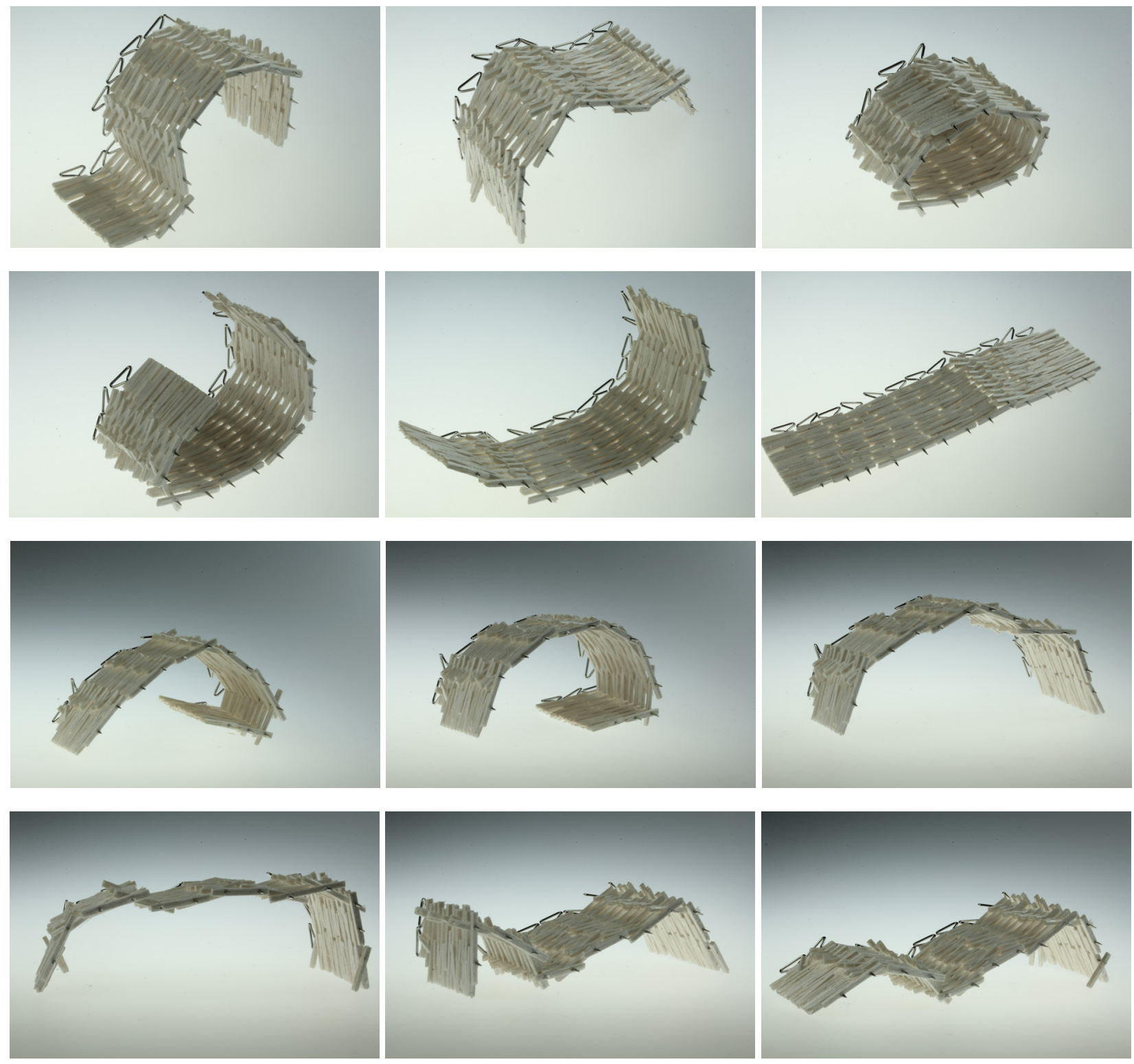

Figure 3.21

Physical models of Iteration $1 B(2)$ that were manipulated by hand to

explore the possible forms. The hand manipulation acts to replace wind on site. This is a small selection of the images and forms possible. 


\section{Affect Reflection}

Case-study analysis raised the significant scale between sculpture as a non-functional medium and architecture designed to serve a functional need. I wanted to explore the relationship between these two fields through modelling and drawn analysis to find a point where function and experience can combine to create a memorable architecture. These tests continue to emphasise my research approach and focus on sculpture as an experience driven design process that is more dominant than the functions of the building. These tests question the traditional functional drive behind architectural design and interact with the surrounding conditions in an attempt to also connect the user with their environment. 


\section{Design Iteration 1C}

\section{A Moving Interaction}

A series of design tests focussed on developing non-static structures with a higher complexity to the pin joint concept in the previous section. Initially I aimed to investigate structural systems that could create a more limitless deformable space.

The process analysed wave and wind rhythms on site as a form finding mechanism to be translated into structures, spaces and programmatic layouts. The transformation of a natural condition that has a short temporal period of change into architecture began to challenge my own preconceptions of movement associated with architectural space. Initially the wave movements were recorded through a process of video footage, physical modelling and then overlay of images of models to create both an oblique and sectional representation of the movement (Fig 3.22). This iterative analysis helped to break down a movement into volumes that have potential for occupation.
The fluctuating methodology between two-dimensional and threedimensional exploration created distinct forms for each phase of wave movement and then brought them together again in one image to reinvestigate the complete sequence. Despite this, the translation of a movement into a static or flat plane opposed the concept of trying to embody a non-static process.

This moment-based analysis of the wave was then translated into a moving shelter concept that embodies the idea of semi-permanence; designed to softly unfurl with the advancing and receding waves. Materiality, perspective and siting were considered thoroughly to explore the relationship between the occupant to the ground plane and the attachment or detachment they would feel to their surroundings. ${ }^{12}$ (Refer to ...)

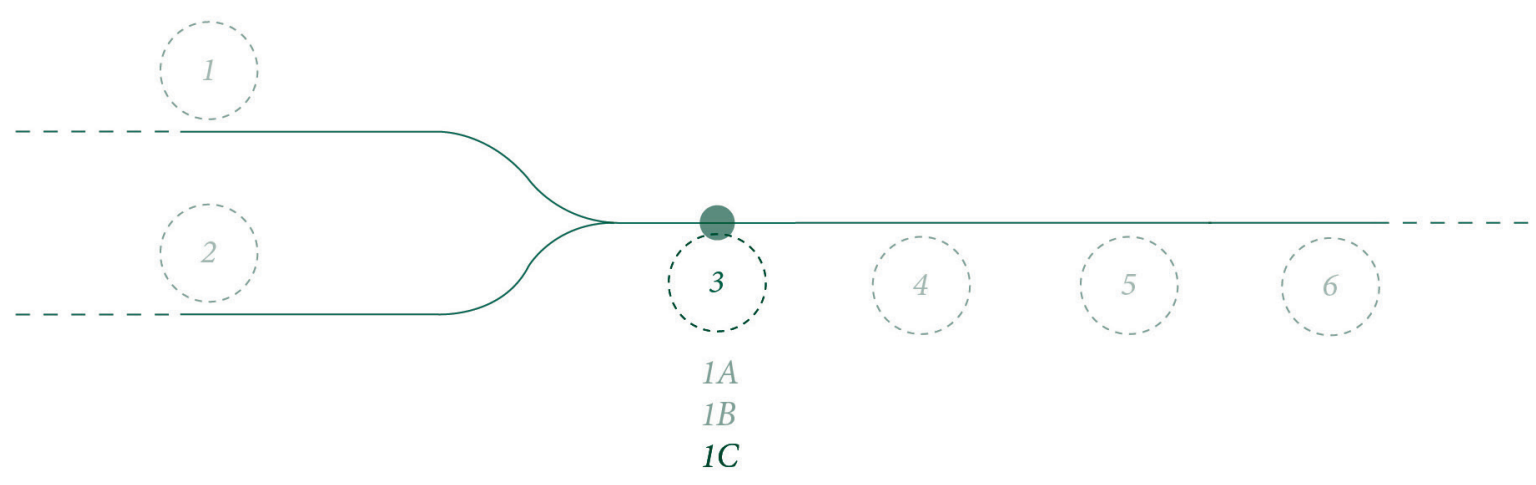


Click image to play video
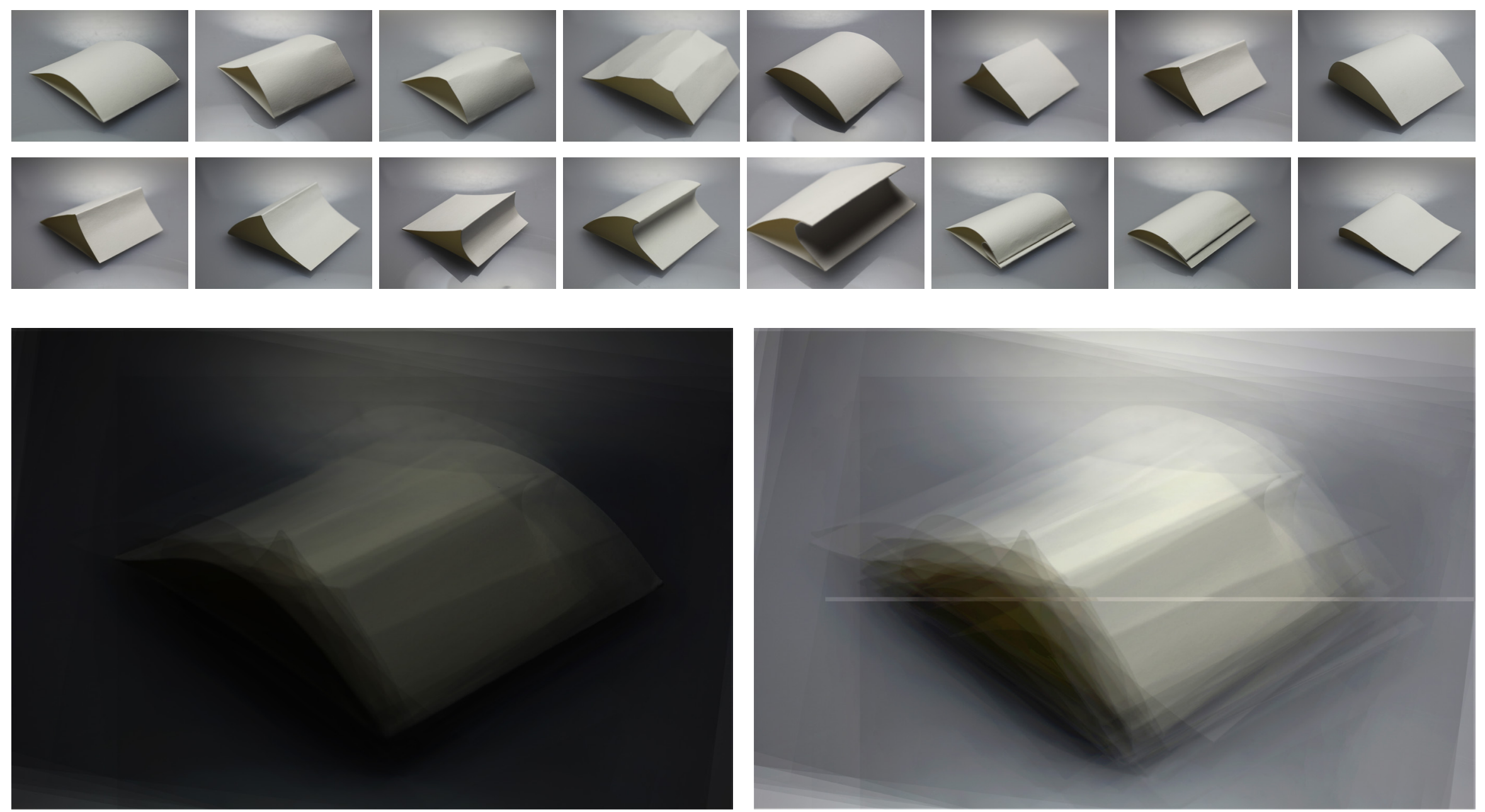

Figure 3.22

Wave breaking video translated into model sequences and overlays.

The 6 second period between breaking waves was split into a sequence of This exercise was designed to break down the short time period of change in models depicting the stages that the wave form goes through before reaching near flat foam on the beach. the natural environment. These models were photographed from the oblique perspective to explore the creation and understanding of movement. 

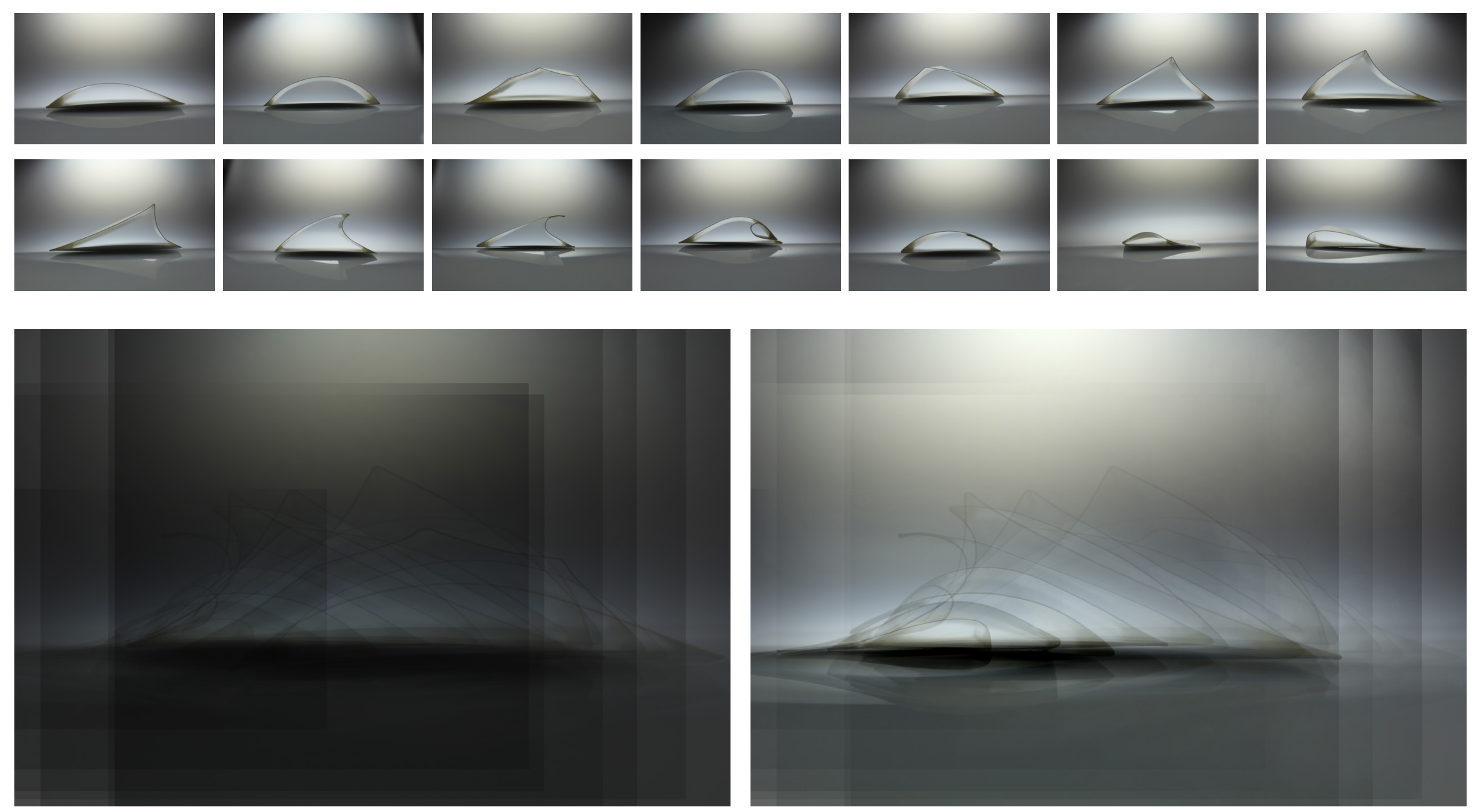

Figure 3.23

Wave breaking video translated into model sequences and overlays.

This model sequence was photographed from the sectional perspective and overlaid to see if it the communicated the same sense of movement as the oblique perspectives.
Both of these investigations were then used as form drivers, as well as system drivers for the building to have a sequence of transformation that relates to the natural context. 


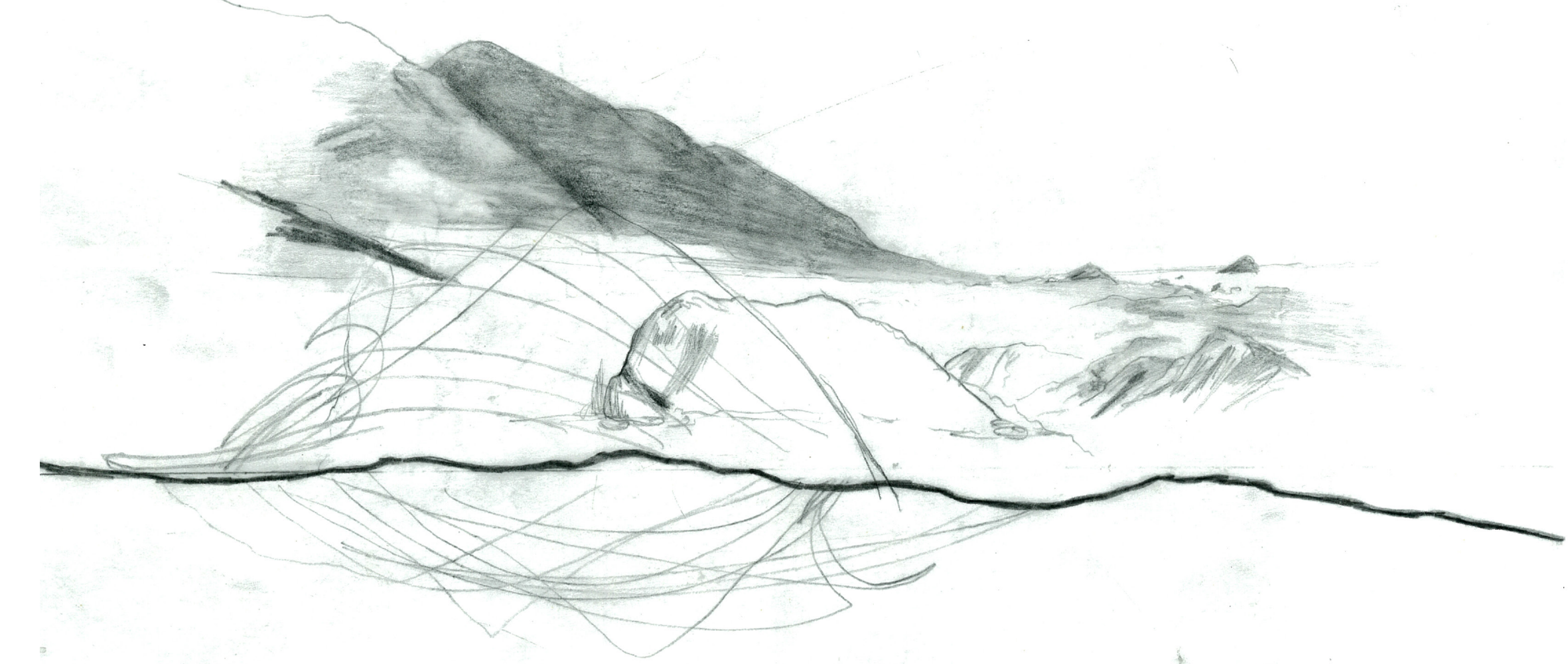



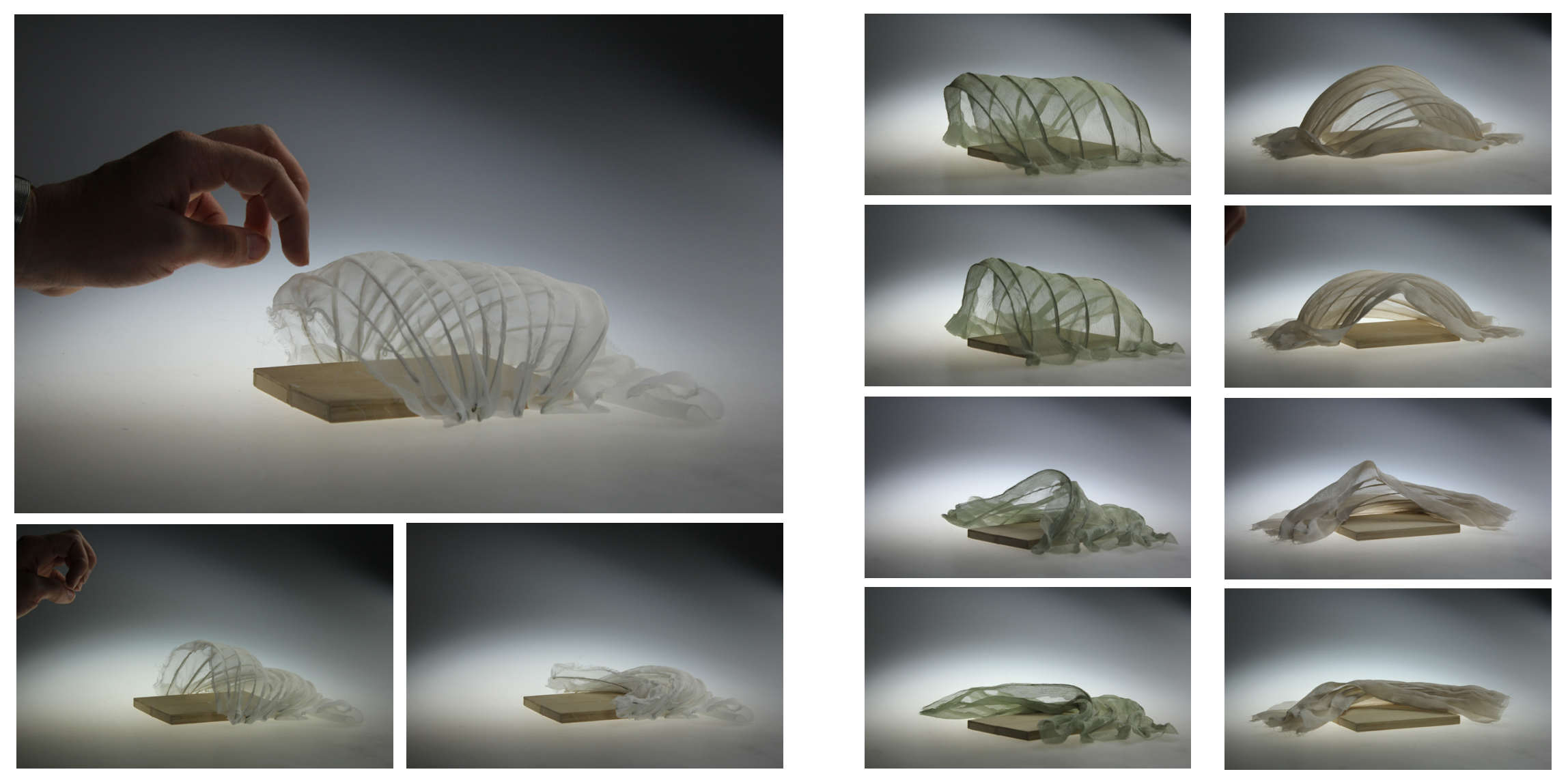

Figure 3.25

Model test one. A small semipermanent beach shelter and base platform.

The form had to be integrated with a system that allowed it to move. I prioritised options that required no mechanical control and could be driven by water. This creates a direct relationship between action and reaction.

A sleeping platform that can be folded smaller forms the base to sleep on. This needed to be solid to be placed in such a rocky and uneven ground

I modelled an unfurling lightweight canopy and visualised it connected to a pulley system to open and close the cover in a constant cycle. When the shelter is inhabited it influences this movement and the shelter can become static. This movement creates many of the shapes that were identified from wave analysis in section, from the high swell point to a flat rushing wave. 
Following this first non-static concept I explored the multiple rhythms that were present in a fern frond when wind acted as the sole exterior force. The fluctuations of the slow rigid interior branches in combination with the faster oscillating outer leaves introduced an interesting interplay between relative periods of movement. This natural movement was again translated into a structural form and programme, both as a series of interconnected modules, and as one large design through the process of stick weaving. ${ }^{13}$

I initially analysed the video footage of the fern movement and linked this to programmatic and siting arrangements for the shelter. This separated plan was not used in the stick-weaving test but was revisited later in the design process as a method of maximising the potential for movement in the programme.

I then tested Bradford Hansen-Smith's stick weaving mode of construction as a malleable and flexible structural alternative to previous models. A range of joints were tested and manipulated to determine flexibility and occupation of space. Stick weaving created an innovative structural system where the whole entity embodies movement. 


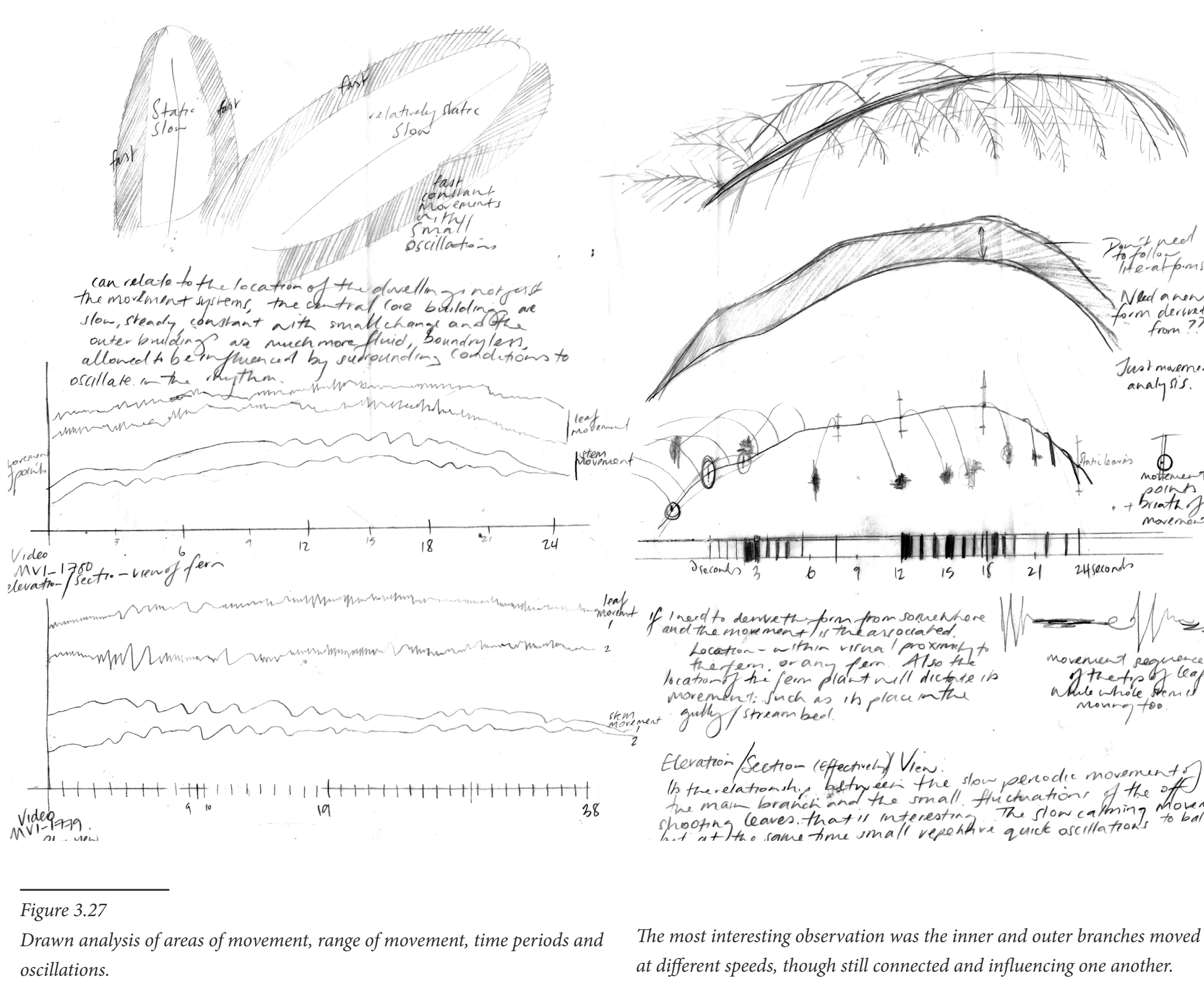




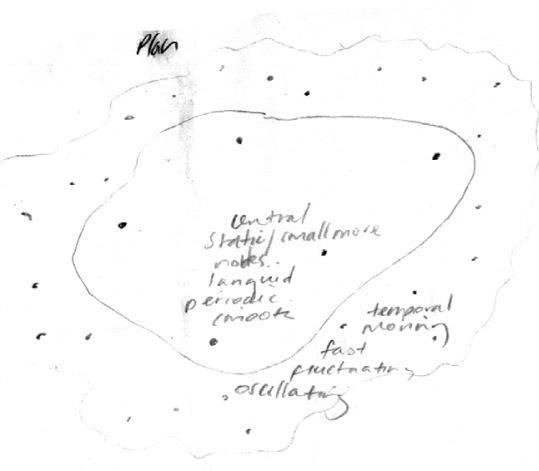

Relationship between central node + small outer nodes.
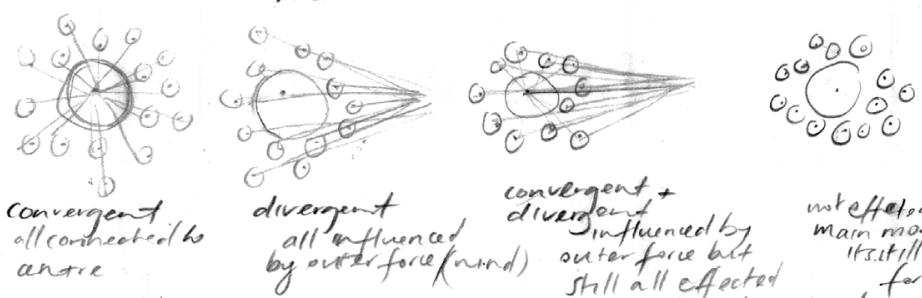

Placement:

Area association, this to programme too or just movement? levels of movement $t$ influence by wind. 'Also the types of change that will be present moth shelter.

placement: onecentral

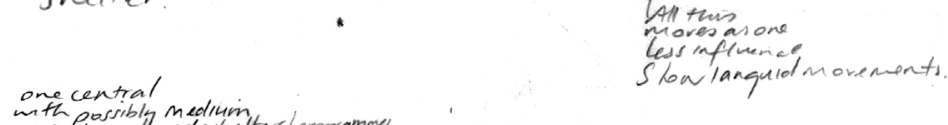

that only the ven outer reaches are inflamed by the wind the

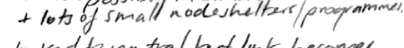

association: linked to central but link becorver
pretty much wold of the extreme nodes, they are separate

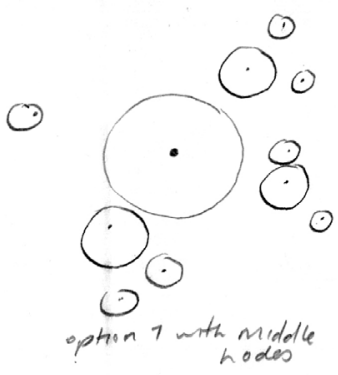

Association:

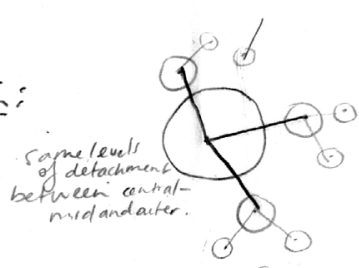

$\varphi$

( $)$

$\odot$

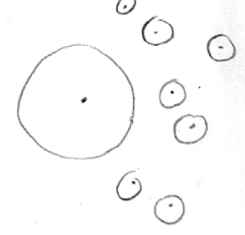

option with post
central +outer

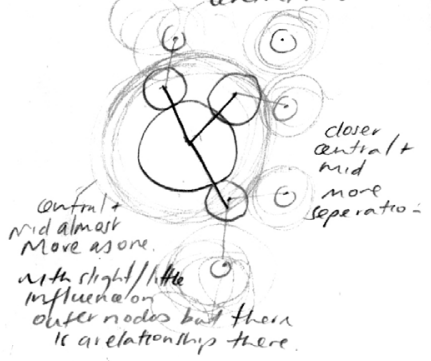

Levels of movement.

levels $y$ influence:

Types of change: as described.

levels of move: central has slight movement/change

To do tomorrow: relate to noon static relate to location
wilderness

levelsitgence: central has stightimfluence \&

develop some form

types fchange: curter lis stow languid anobtrusivechange that mid has same inficenceas central sochanges
with central but with sight infucencebyond to separate from central

outer has no relationship movementehange-wee

outer has no relationship movementichange-wie
moves own accord but as both are influencerlbs
extemalconditions there may he some simians

Figure 3.28

These diagrams display the conversion of the inner and outer leaves and their connected yet separate movements into a programme and arrangement of shelters.
The programme explores a layout with one central shelter (spine) and a series of smaller shelters surrounding, which are either are all connected to the central space or another external point, (e.g. facing a direction or linking to a path).

98 
The stick-weaving structure was developed into a malleable model large enough to manipulate. I began to explore the inhabitable space, as well as the extremes of how this constructed system can transform. The fourway joint was flexible when not connected to other joints but in the lattice became much harder to manipulate, and did not properly reduce down to a flat compact lattice in both directions. (Refer to 3.29) Despite this, with more force I was able to manipulate it into many different forms ranging from; one direction pressures which resulted in symmetrical arrangements, to multidirectional pushing and pulling to create unsymmetrical inhabitable spaces within and around the structure.

Stick weave test two was constructed with $3 \times 3$ stick joints for more flexibility and malleability (refer to Figure 3.30). As predicted this generated a less symmetrical system with 'mountains' and valleys' created by the trijoint. This meant that as a system it was harder to visually understand but there were a wider range of forms created. This iteration was more flexible and only required little force to make it into any shape needed. The vertical pressures were able to reduce this one into flat lattices in both ' $x$ ' and ' $y$ ' directions. I think that this system was more successful in trying to achieve the non-static function that this iteration was focussed on. The transformed patterns and spaces to be occupied are a greater variety and the structure would be able to be easily changed by the wind on-site. Due to the increased flexibility and the lack of symmetry the interior and exterior spaces were blurred, therefore offering a more interesting relationship between architecture and environment.

The three-weave was developed into a shelter concept through a series of sketch plans that tested transformability, occupation, protection and siting at the most basic level. (Figure 3.31-3.33)
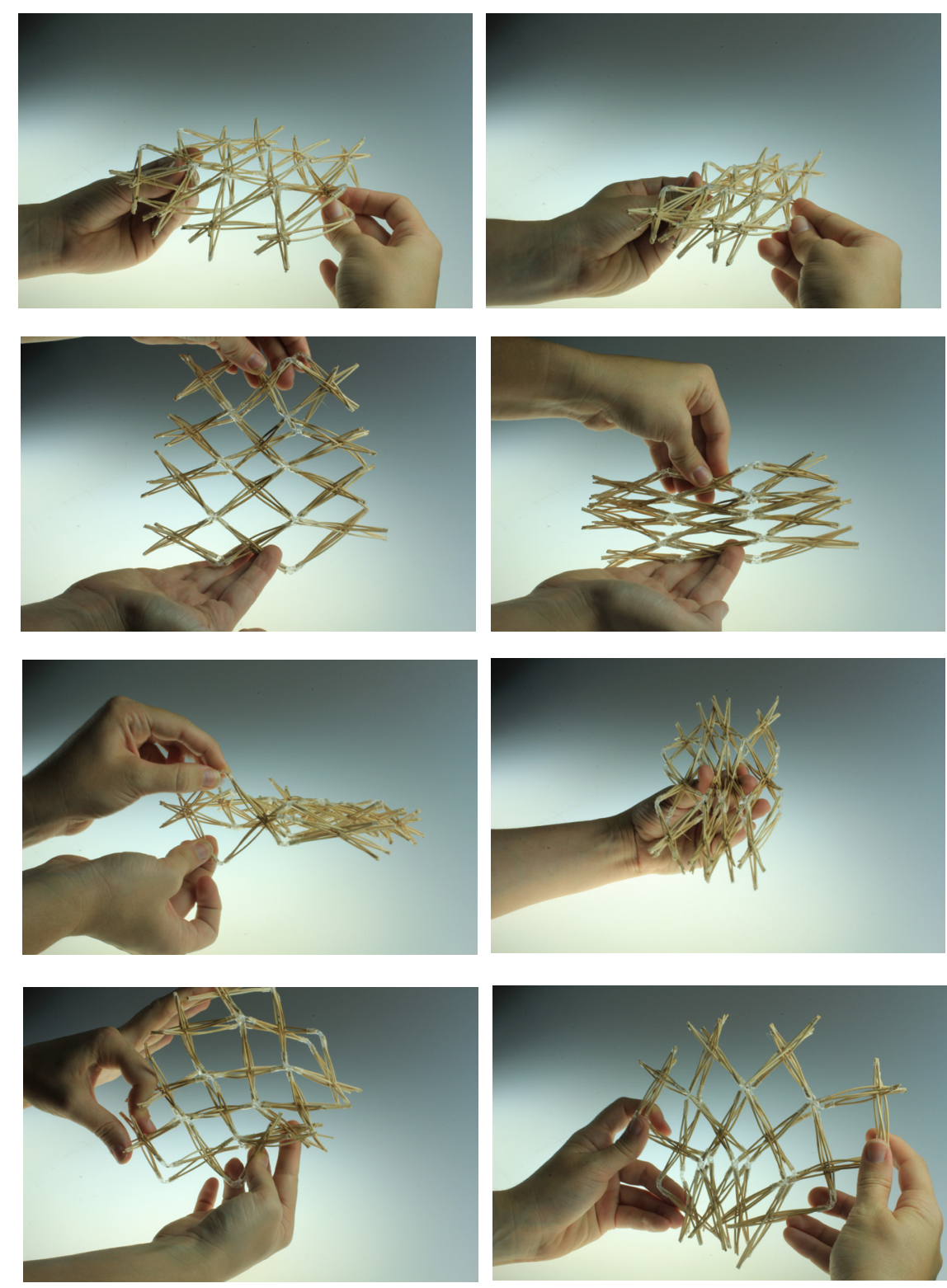

Figure 3.29

Stickweave test one, four joint weave. 

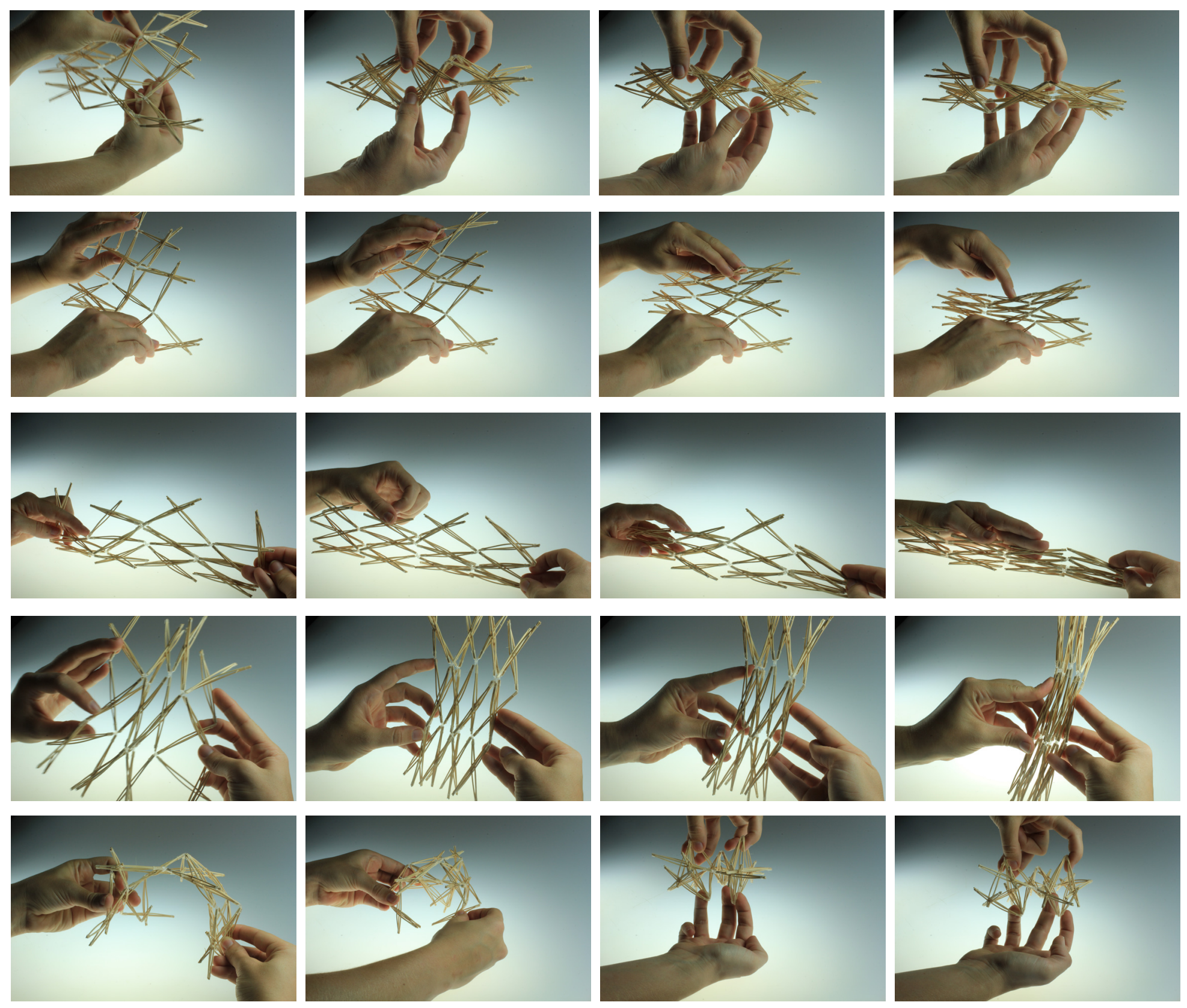

Figure 3.30

Stickweave test two, three joint weave. 


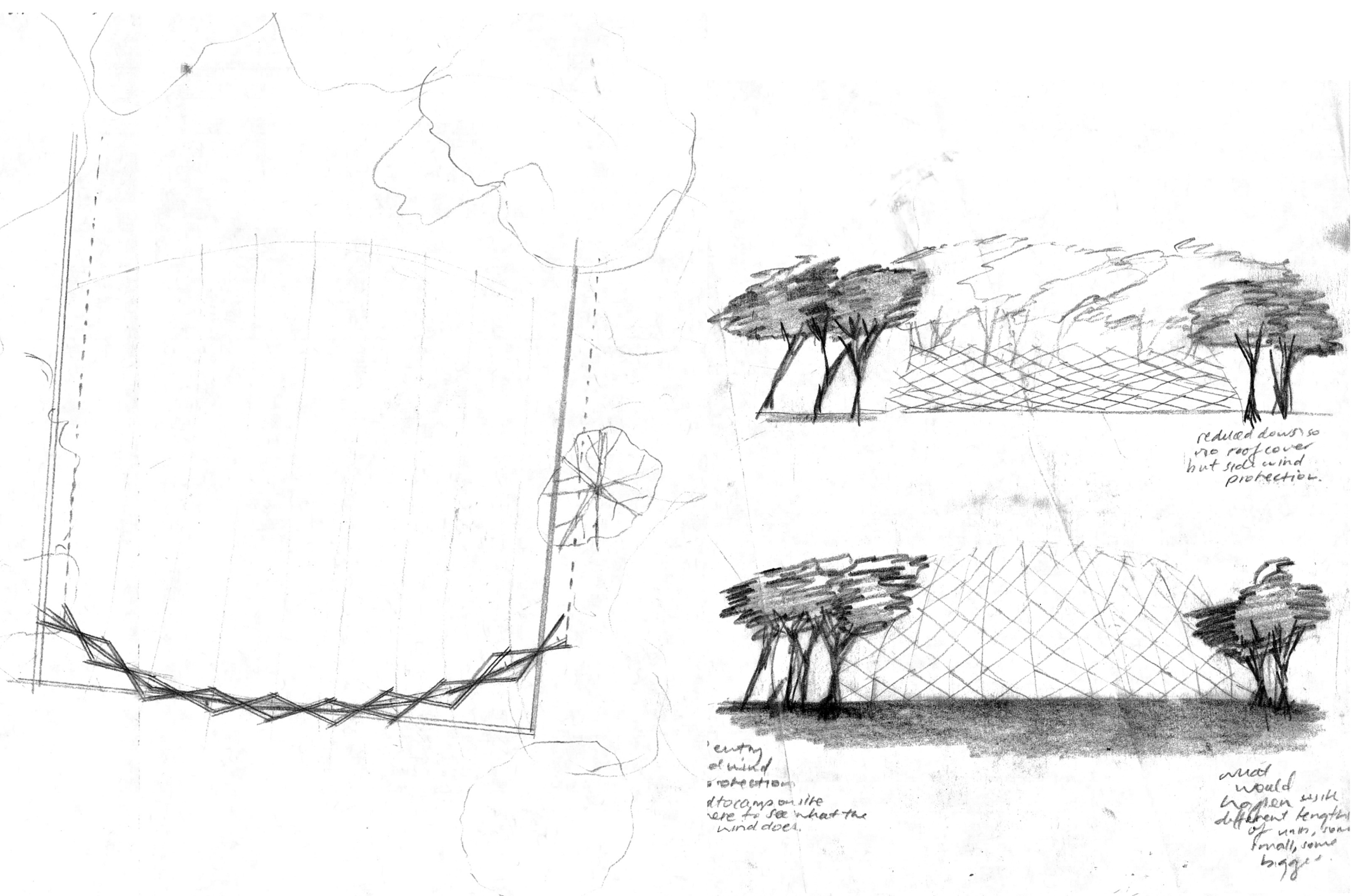

Figure 3.31

Stick weave concept plan for a simple shelter that transforms based on the conditions and give weather protection when

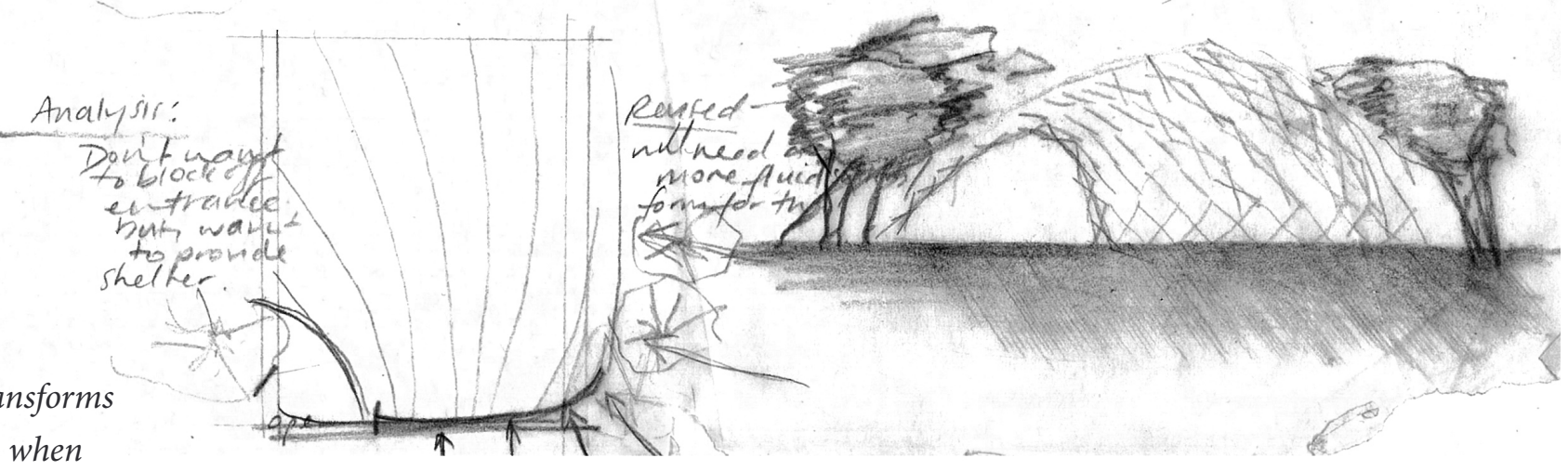
required. 
Location: Already Establuned

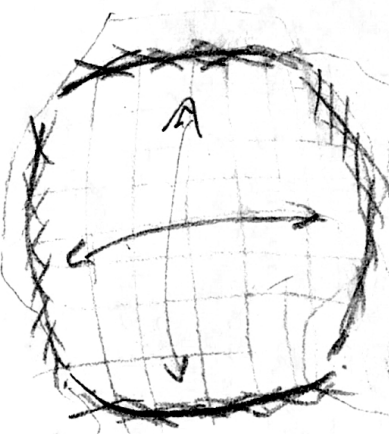

could yen form the structure solthest it could bunch in any direction?
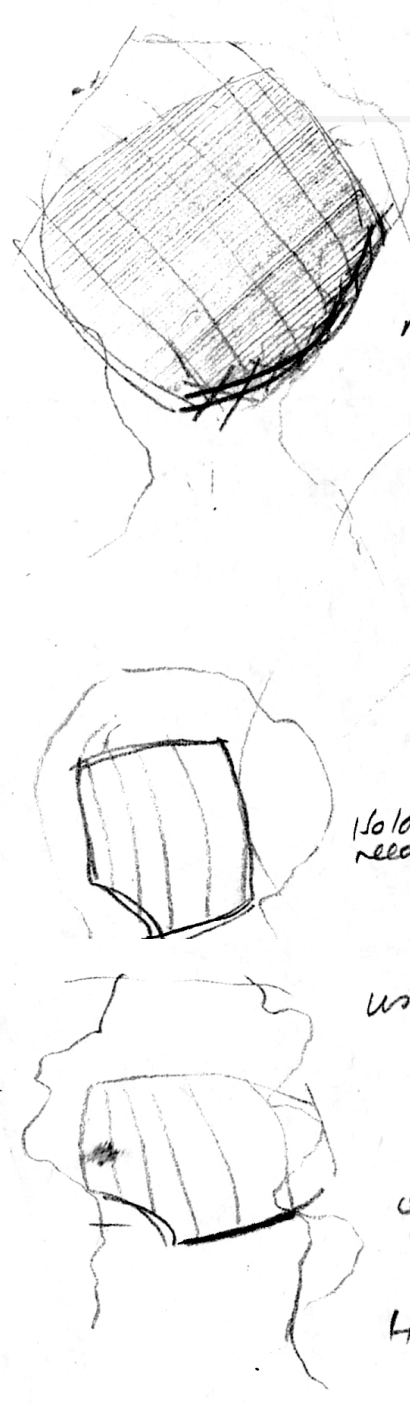

$$
\text { roputedto from }
$$

$c^{2}$ on edge $f$ bwahline

interesting that buscilime "associate. one a gouge t closer to the lowest. orel frowns snow.

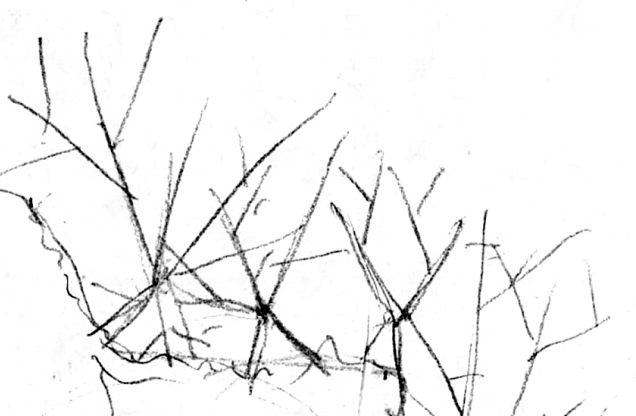

7 nimedaredixed

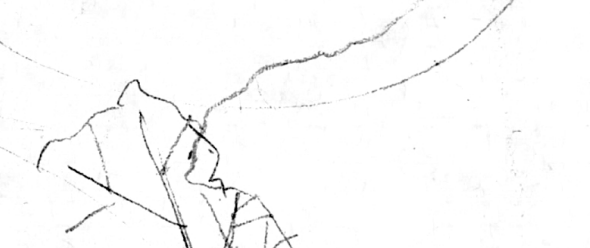

$$
\text { isolated from all ides }
$$
through

using trees as path

$$
\begin{aligned}
& \text { nil hakim owe } \\
& \text { read, sonvedges } \\
& \text { - rtransientipaces }
\end{aligned}
$$

filose proxinim between

campers and ruler.

How to segneflingene or locale tisinbase

Figure 3.32

Second concept plan that explores deformation of the weave structure in two directions to increase weather protection.

102 


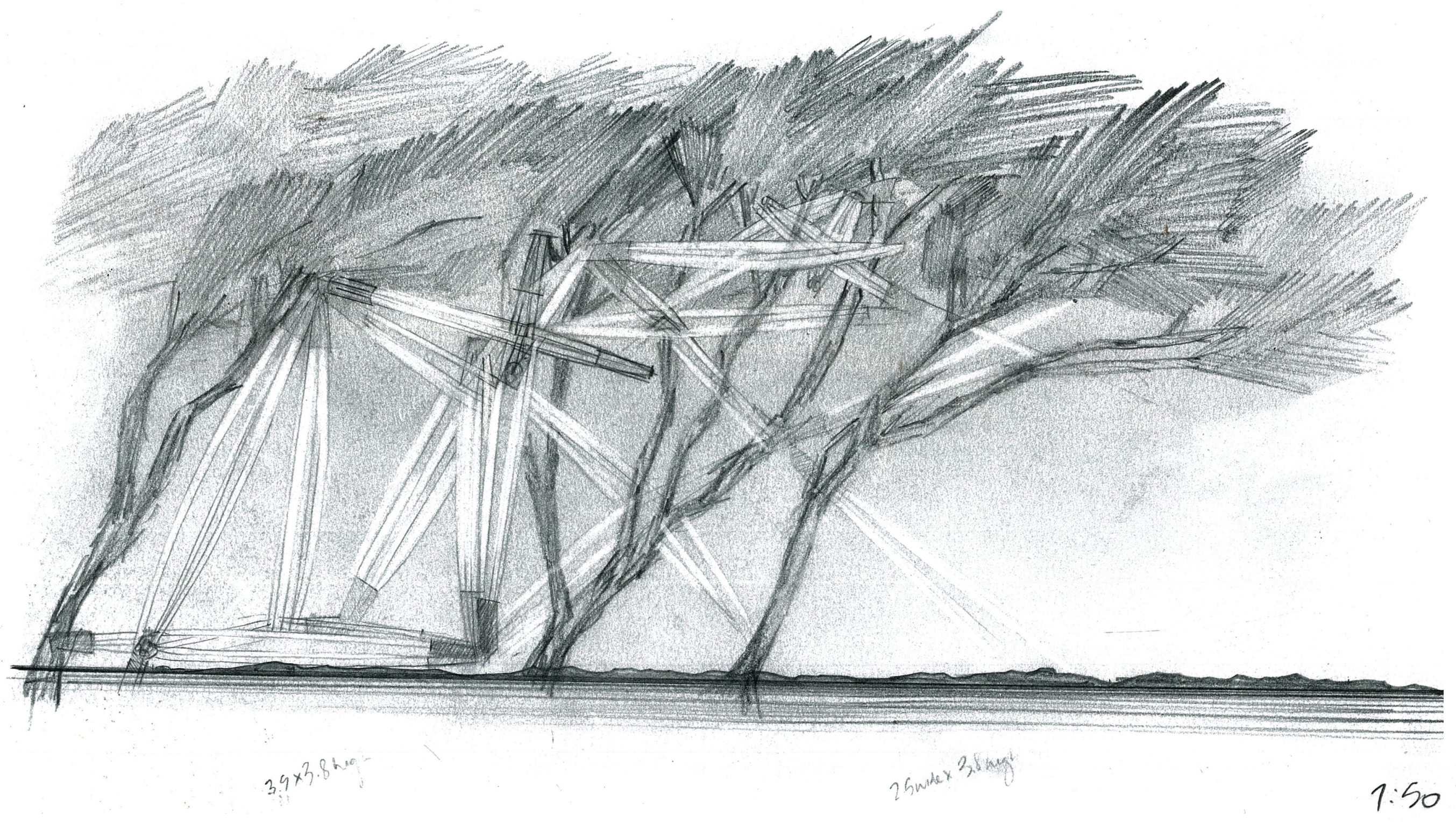

Figure 3.33

Stick weave inhabits the landscape 


\section{Non-static Reflection}

The mechanical or system based structural concepts all embodied movement, connection with the surroundings and created inhabitable space. Programme development was limited but the process explored in the fern frond observation created a new design methodology verging on biomimicry. By translating the interactions present in the landscape into a programme it shifts the drive from the designer to successful natural systems.

\section{Phase One Discussion}

In conclusion, each of these separate iterations; non-static, affect/experience, and wilderness; were designed to focus on one theoretical field through research, design and reflection. Despite this, common themes have begun to emerge; for example, the interaction between wind and architecture is one trend present in all three sections. Movement, manipulation and the use of architecture as a filter to alter experience is also present in all areas. The next phase will collaborate the most successful ideas into one design that resolves the programme, siting and structure to a higher level.

The next phase experiments with dynamic movement to form a less conventional mode of inhabitation. This system can deform in both the ' $x$ ' and ' $y$ ' axis (developing the ' $z$ ' axis also) to retract design control and allow a freedom of interaction with wind as discussed in affect/experience. This semi-permanent wilderness shelter is designed to create a constantly changing high impact experience of a new dynamic structural system that interacts with the most captivating qualities of our natural environment. 


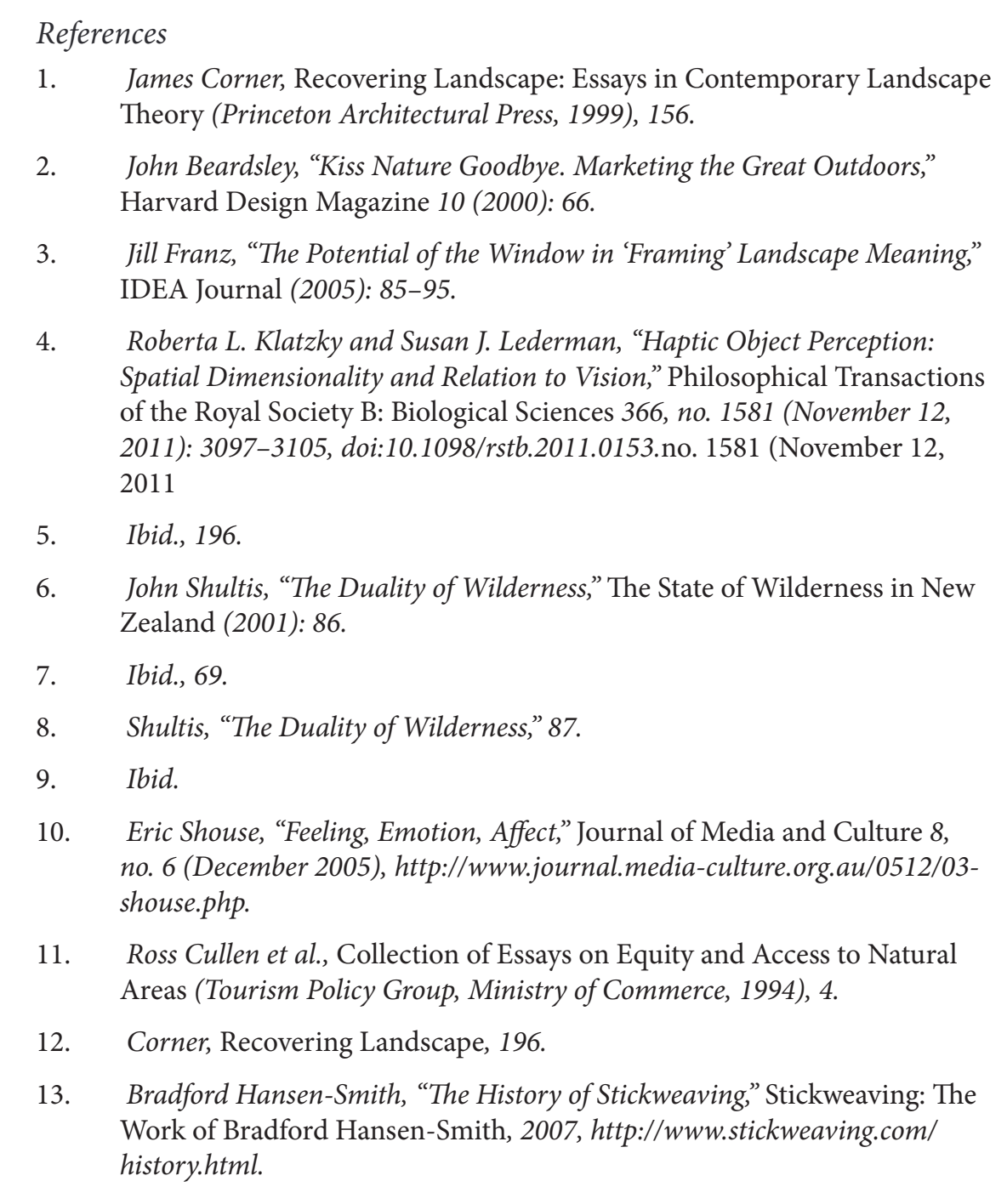


${ }^{106}$ | 


\section{PHASE TWO CONCEPT DESIGN}

The literature review in Chapter Two proposes that increasing connection and awareness of the relationships between architecture and environment can be achieved through movement in relation to the affect theory of tending. This conceptual design phase will explore how architecture detailing can relate to Nigel Thrift's theory on the manipulation of space and time as a powerful means of generating affect. Structural development of the nonstatic mechanism and a series of drawn tests into occupation, programme and their relationship to a specific site have been combined to form Design Iteration Two.

$-4-$

Investigation

Space, Time and Affect

Design Iteration 2

Form - Structure

Sited Occupation

Phase Two Reflection 


\section{Investigation}

Space, Time and Affect

Thrift talks about slow motion film and how it allows the opportunity for an awareness of the most minute details of a scene that would usually be below human conception. ${ }^{1}$ Case studies such as Kinetic Rain by ART $+\mathrm{CO}$, display how the sculptural field is exploring slow motion through mechanical computational means. A piece of art like this is developed in a context, which aims to make invisible the technical support that generates the effect. Instead, in highlighting the relationship between wind and architectural form this thesis makes visible the forces that drive to movement; to intrigue, inform and educate. The non-static structural form has the potential, depending on the stiffness of the joints to translate a strong wind that interacts with plants and trees in rapid gusts of movement, to a slow responsive transformation of form. The role of the occupant as an inhabitant who can alter the form of the building to match their requirements of space while it is still interacting with the conditions, physically reveals the largely invisible reaction between wind and building and now occupant too.

Movement not only relates to the theoretical context but also is a pragmatic response to the semi-permanent glamping brief. Temporality requires simple mechanisms that can be set up and removed with ease as well as transported to the site with ease.

\section{Design Iteration 2}

Form - Structure

This iteration sought to explore options for interactive movement within the structural system. The stick weave was developed through an intense period of iterative modelling to develop alternative arrangements, joints, connections, and to explore options for watertightness. (Refer to Fig 4.1-4.9)

Initially physical modelling explored the potential for the structure to deform to greater extents in the $z$-axis. This investigation acts as a testing sequence to see the range of forms and structures possible and how they would deform and interact with conditions on site. Increased movement of the vertical axis opens up the interior space within the structure to reveal options for inhabitation. A series of models were constructed that alter the number of joints, their arrangement and the number of stick trios in each. Physical modelling is successful as it allows rapid development of the structure, I was able to randomly create new combinations directly developing from the previous iteration.

Consequently, this process identified a number of key considerations for the structural design:

- The ratio between stick size and length for each of the joints determines how easy it is to transform the model.

- Increasing the number of joints increases the flexibility of the structure

- Longer sticks curve and create a lightweight structure that is extremely transformable

- Arrangements that act as weaves or are circular are the most transformable.

- Geometric shapes such as squares and rectangles become too rigid

This iterative process revealed that there are limitless arrangements and forms. The occupation and use of space should be developed and then dictate the mechanics and form of the structure.

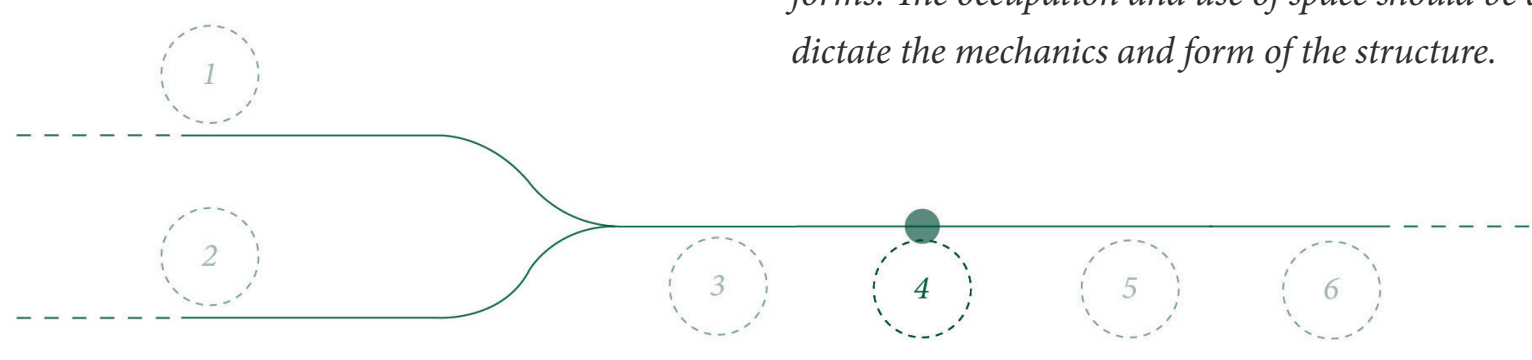



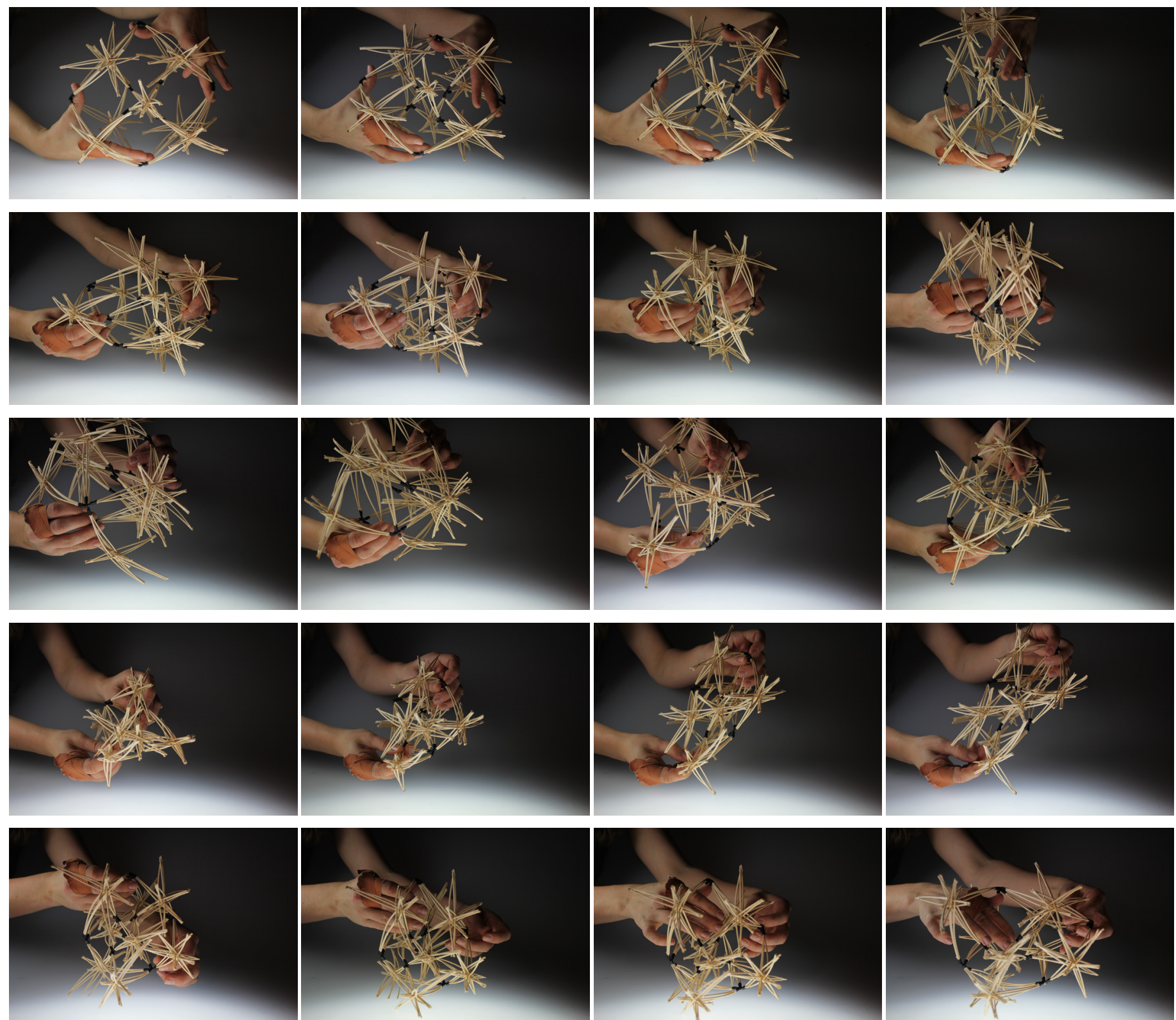

Figure 4.1

Weave with five joints connected around one central joint.
This test reinforces the idea that the stickweaving module can transform in $x, y$, and $z$ axis and offers variant options for internal volumes. Although, there are some limitations with the design that mean it is not compressible in all axis. 

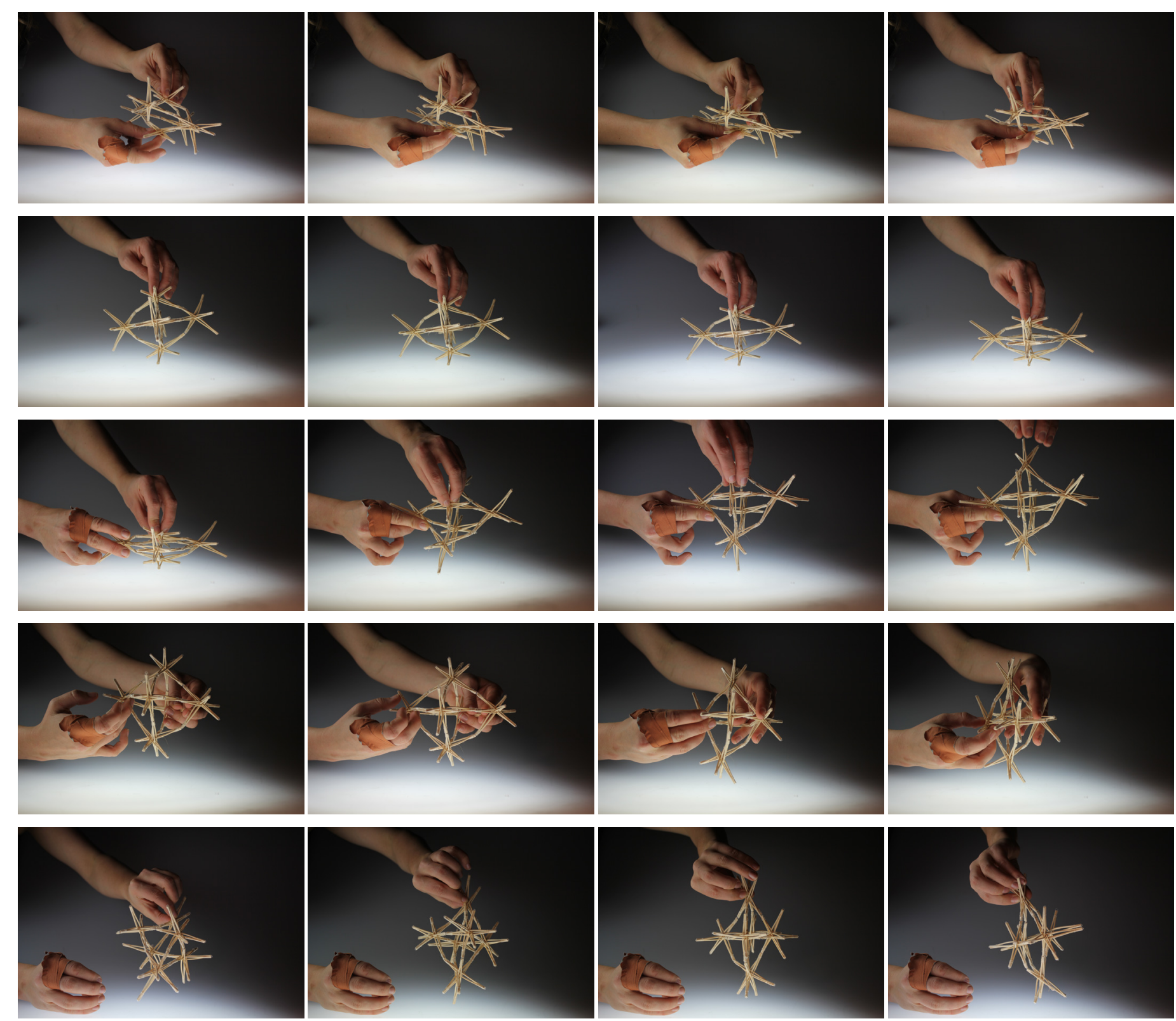

Figure 4.2

Six joints each with four trios. This is the first arrangement where the system consists of only singular connections. Each joint is connected to the next only 110 by one point to another, instead of four points connecting.
As a result this model is even more restricted in its movement than the previous. The only directions that it can deform in is inwards as a whole, and outwards as a whole (shrinking and expanding). This experiment lacks the regularity of the first flat stick weaving iterations and so is less effective as a movement system. 

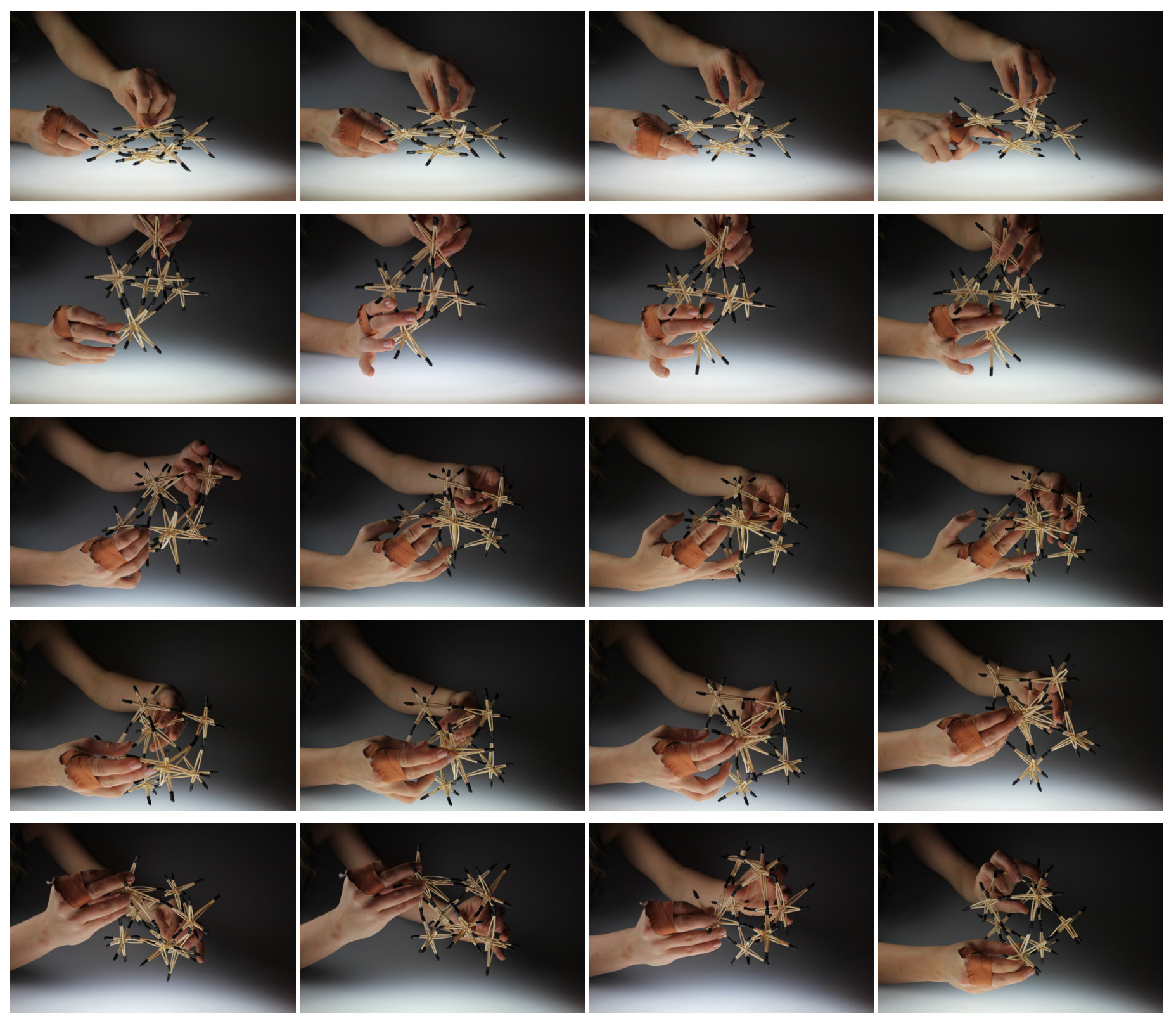

Figure 4.3

The intention with this model was to create another more complex version of the previous iteration with longer sticks to form the joints, and each joint has five trios instead of four.
This model has much the same movement as the previous but with more potential for forces to move the structure and interact with conditions on site. The structures that I deem the most successful have joints that combine four points. 

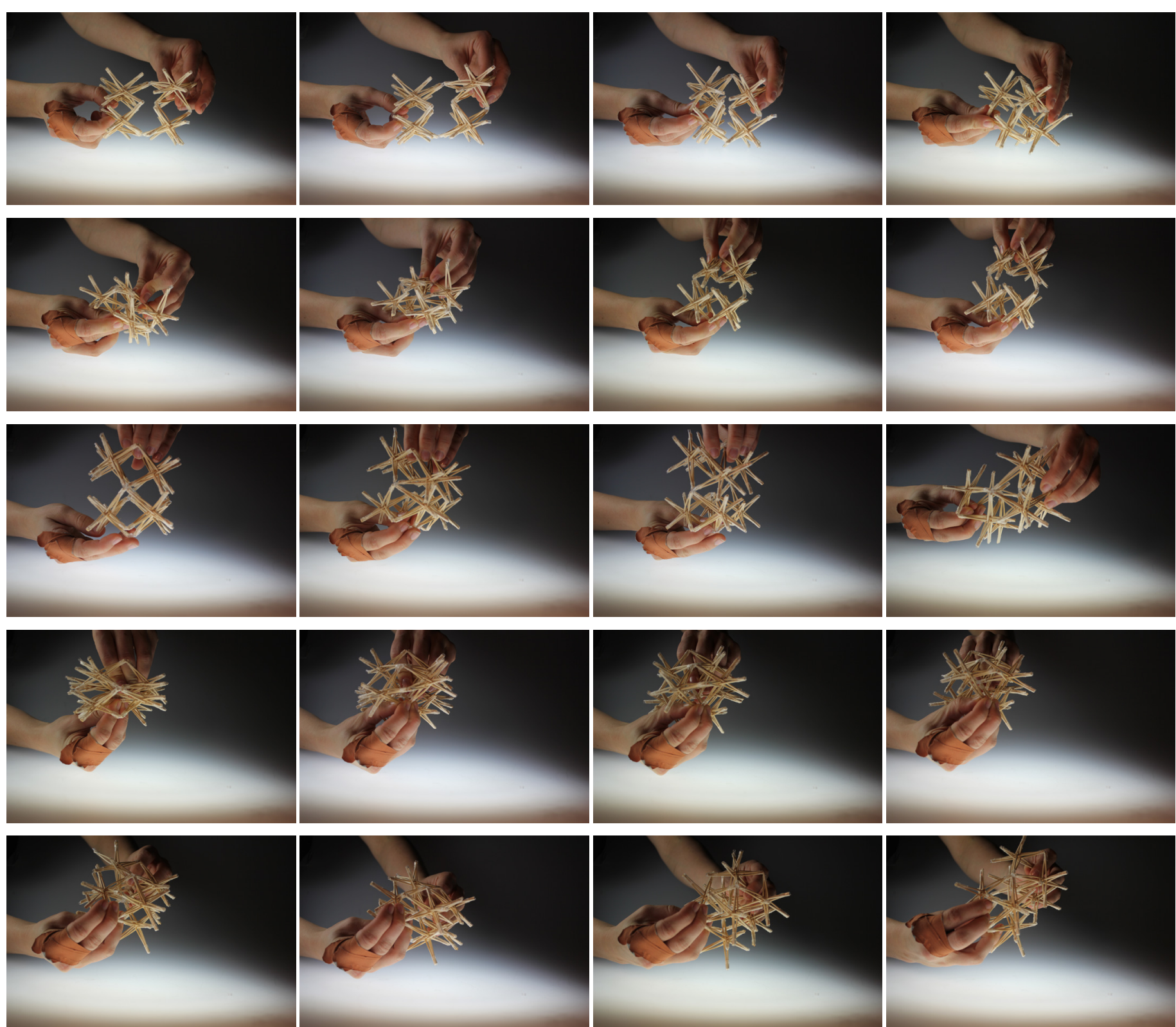

Figure 4.4

This test started with the same connection as the flat initial stick weaving iterations and then joining the outer points but leaving the inner space free. Shorter lengths of thicker cane were used to form smaller units.
This method of connection means that the points are not linked in the middle across, but linked vertically move and react differently to pressure. The form does not have the same success as other trial forms as it remains a relatively rigid structure and does not deform much from its resting shape. Thicker shorter sticks also restricts the movement further. 

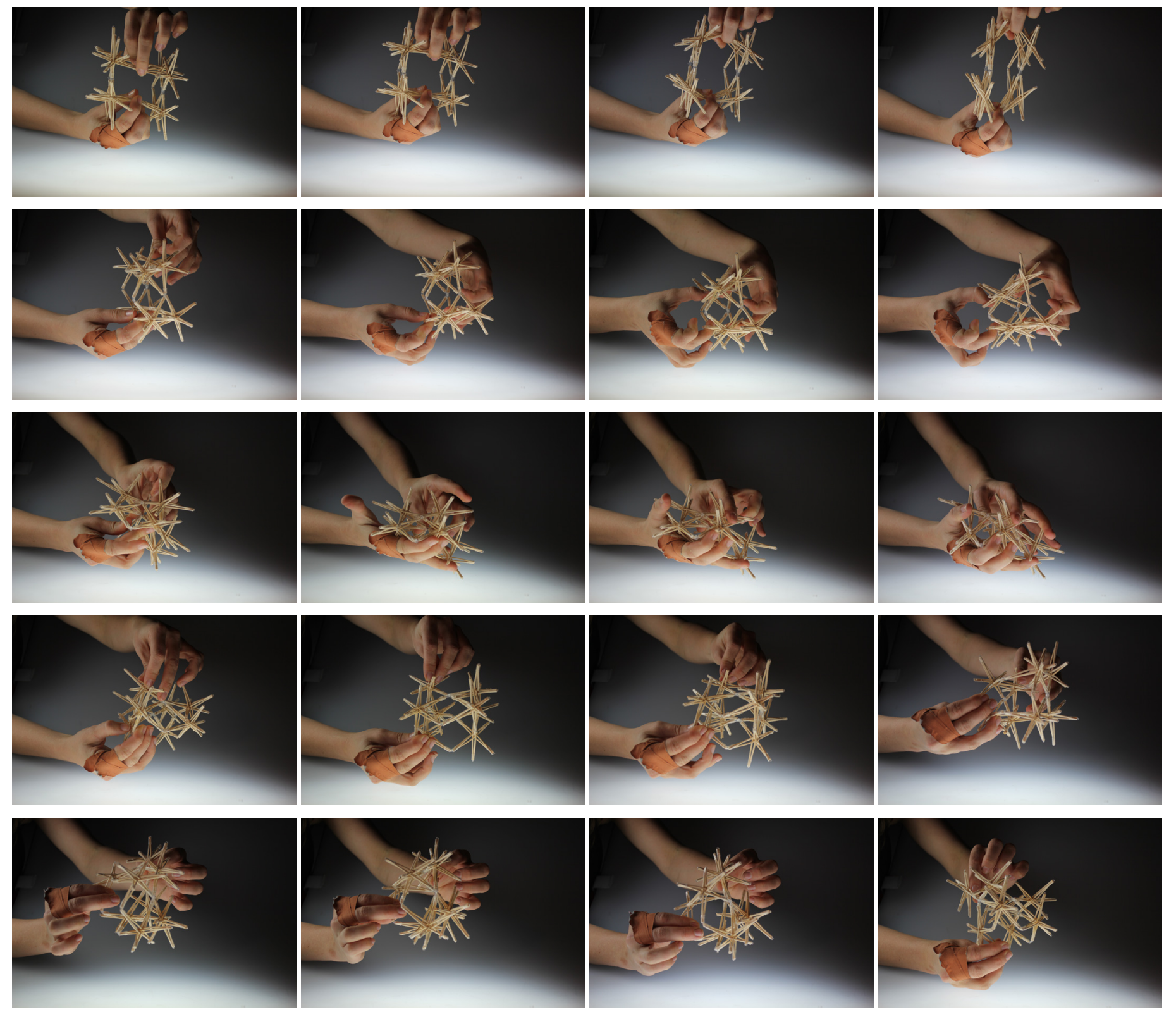

Figure 4.5

The intention for this model was to create a geometric form that responds to more archetypal expectations of space, namely with a rectilinear internal space.
Again as with the previous test this one lacks the ability to deform much from its resting state and is not as successful as the other iterations.

Compression is restricted to the axis shown in centre row of images. This lack of deformation undermines the idea behind the stick weaving structure as a method of creating a form that is collapsible and easy to transport. 

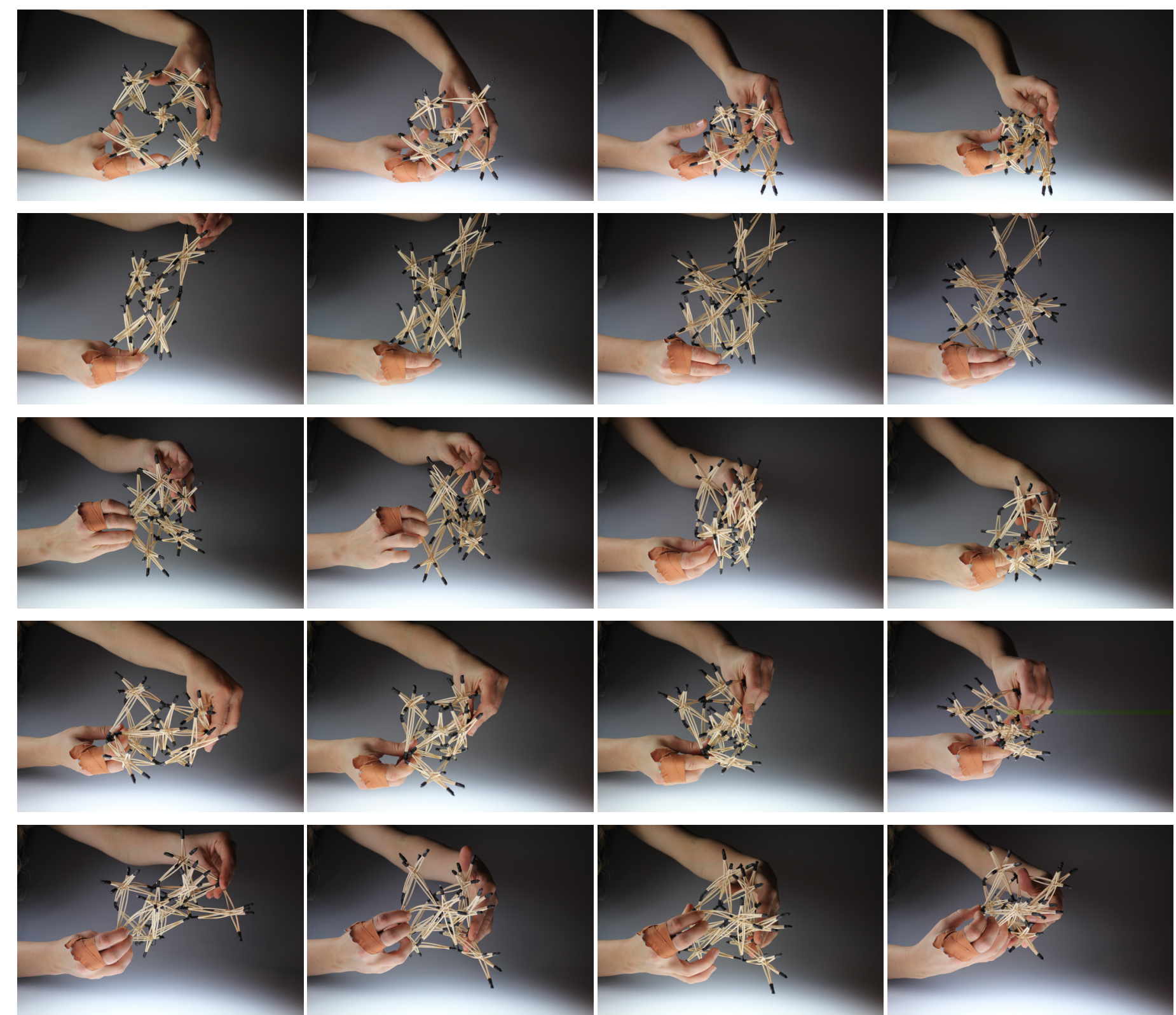

Figure 4.6

This iteration was created to test the same structure as the first test but with a four trio joint instead of five to reveal the differences between the two.
This four joint surprisingly created a more rigid unit than the five. It could be due to the differences in lengths of the joints, but as this was minimal it seemed to be as a result of the rubber joints not having as much give. 
Along with this structural sequence explorations into how a transformable object can be enclosed and the interior kept watertight were modelled physically. Two alternative attachments were tested involving solid and flexible skin options. The first rigid skin is fixed to the structure separate from the joints and allows flexibility through weave sliding movement (refer to Figure 4.7). This does not need to be rigid and would be successful with a flexible membrane option but no matter where the enclosing fixings are it will limit the movement of the weave. The addition of a rigid membrane also provides a greater surface for wind loading and introduces issues such as wind uplift.

The second stretchy fabric allowed flexible movement but through tension restrained some more extreme transformations (Figure 4.8). This iteration was designed so that when the structure transforms the fabric skin either becomes more transparent or opaque depending on the direction of stretch. When sited this would alter the participants interaction with the environment through a visual and tactile link to the exterior. The connection between wind and vision challenges understandings of the atmospheric condition. Critically this option would require watertightness at the moving joint, an impossible tack.

A number of issues were identified for both skin options. There is no insulation and membrane fabric provides little heat retention. Secondly, the fabric exterior skin allowed movement but as the structure was encased it did not provide much visual variation. Inner structure has the potential to dramatically increase understanding of how the system works as the participant enters the shelter, but an exoskeleton would offer more instant visual understanding of the structure on approach. A third sliding fish scale skin with a vacuum for watertightness was proposed as a rigid but transformable alternative option. Regrettably after consulting with industry professionals and thesis advisors, this level of complexity is outside the scope and time limits of this thesis.

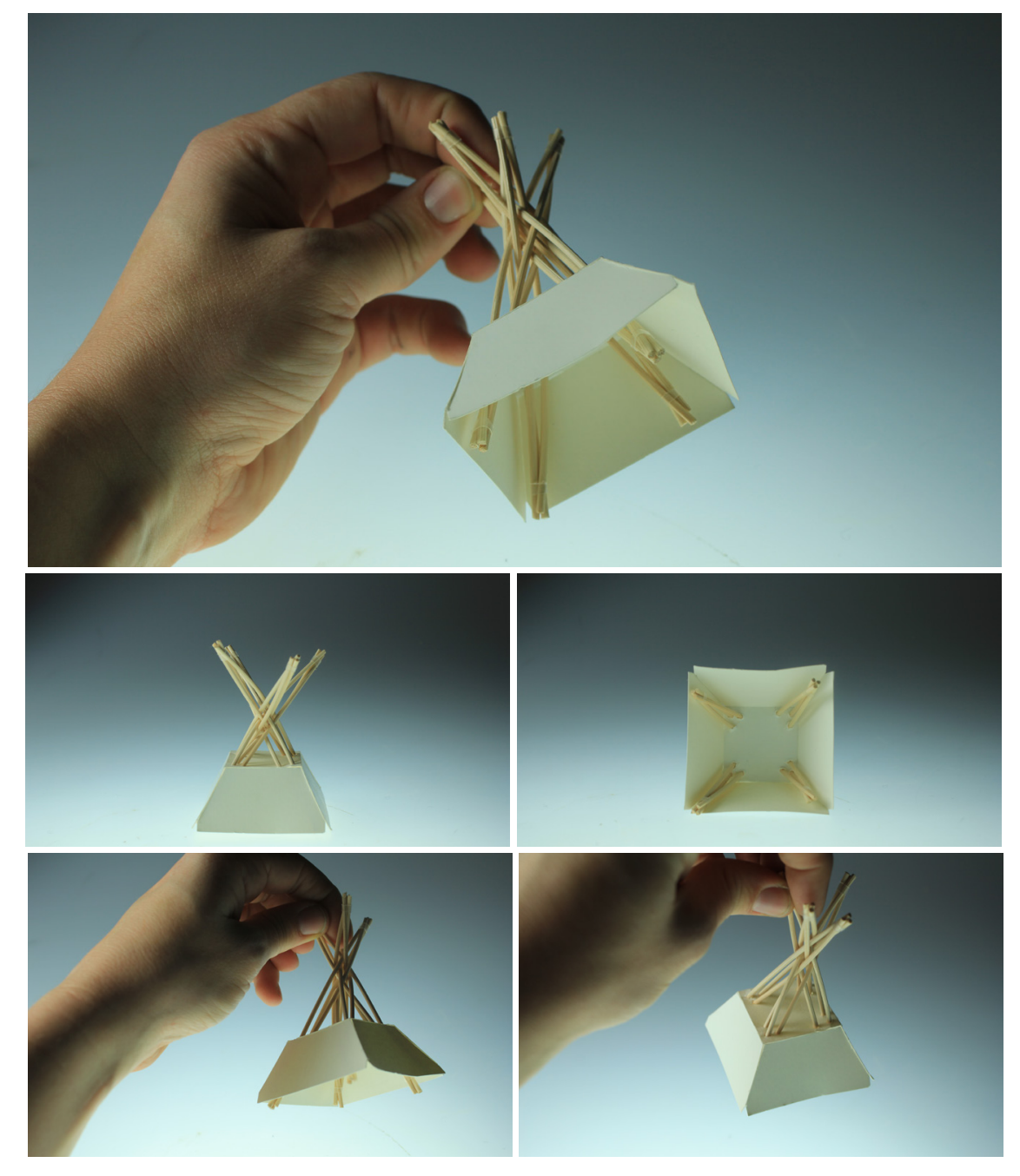

Figure 4.7

The intention with this model was to create a skin positioned so it doesn't need to be sealed at the joints but still allows the stick weaving structure to move. 

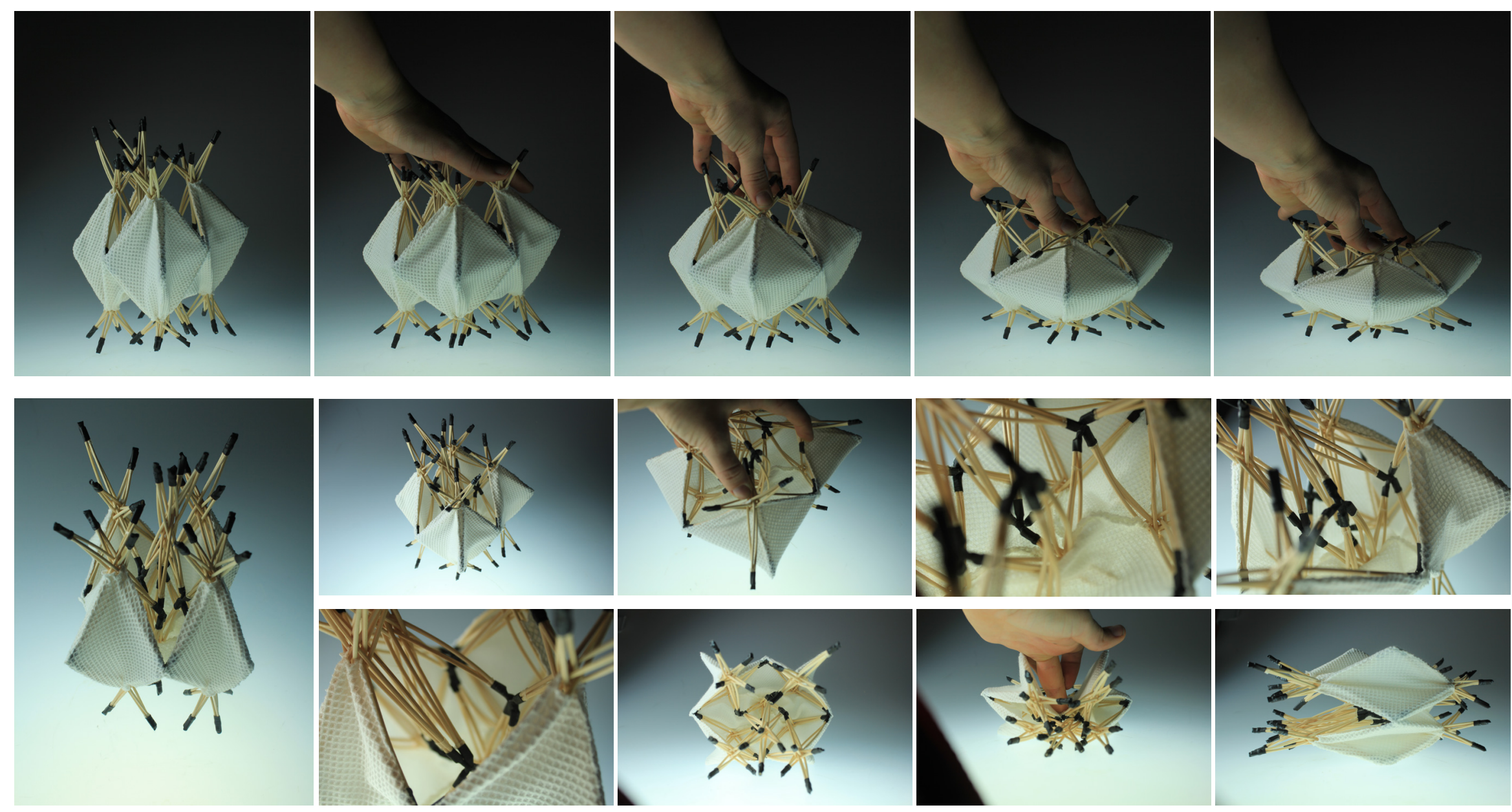

Figure 4.8

This model explores methods of creating a flexible and stretchy watertight skin for the stick weaving structure that has room for movement.

The external skin allowed movement but as the structure was incased it didn't provide much visual variation. 
A 1:1 scale structural prototype was constructed to bring some resolution to binding methods, flexibility of materials, and to observe how a person can interact with full scale joints (refer to Figure 4.9). The structural development returned to the flat weave with each joint consisting of three trios. This option is flexible and inhabitable. Bamboo was selected as a cheap and structurally sound material to build the model. Different diameters, and splitting of sticks were tested to insure enough flexibility and curve for the weave joint. This up scaling reinforced how transformable this weave structure is but did not bring resolution to the joints as a simple rubber tubing and cable tie combination worked perfectly.

A 1:2 scale model tested the bamboo structural binding process from the 2010 Bamboo Wing building designed by Vo Trong Nghia. ${ }^{2}$ This construction method was tested for the stick weave as shown in Figure 4.10, with 9 instead of 3 sticks for each 'trio'. A process of binding called whipping replaced the role of the split wet cane construction and rubber joint options to fix the thinner stick members together in bunches of three. This resulting model was a more flexible weave joint but with no additional value to the original trio method.

This complete structural development revealed that the most simple three trio flat weave was the most deformable. The scale of the joints can be enlarged so people occupy the space within the structure, or reduced to occupy the space beneath so the structure acts as a canopy. In the next stage of development the three-trio is tested with a programme to explore different ways to occupy this structure.
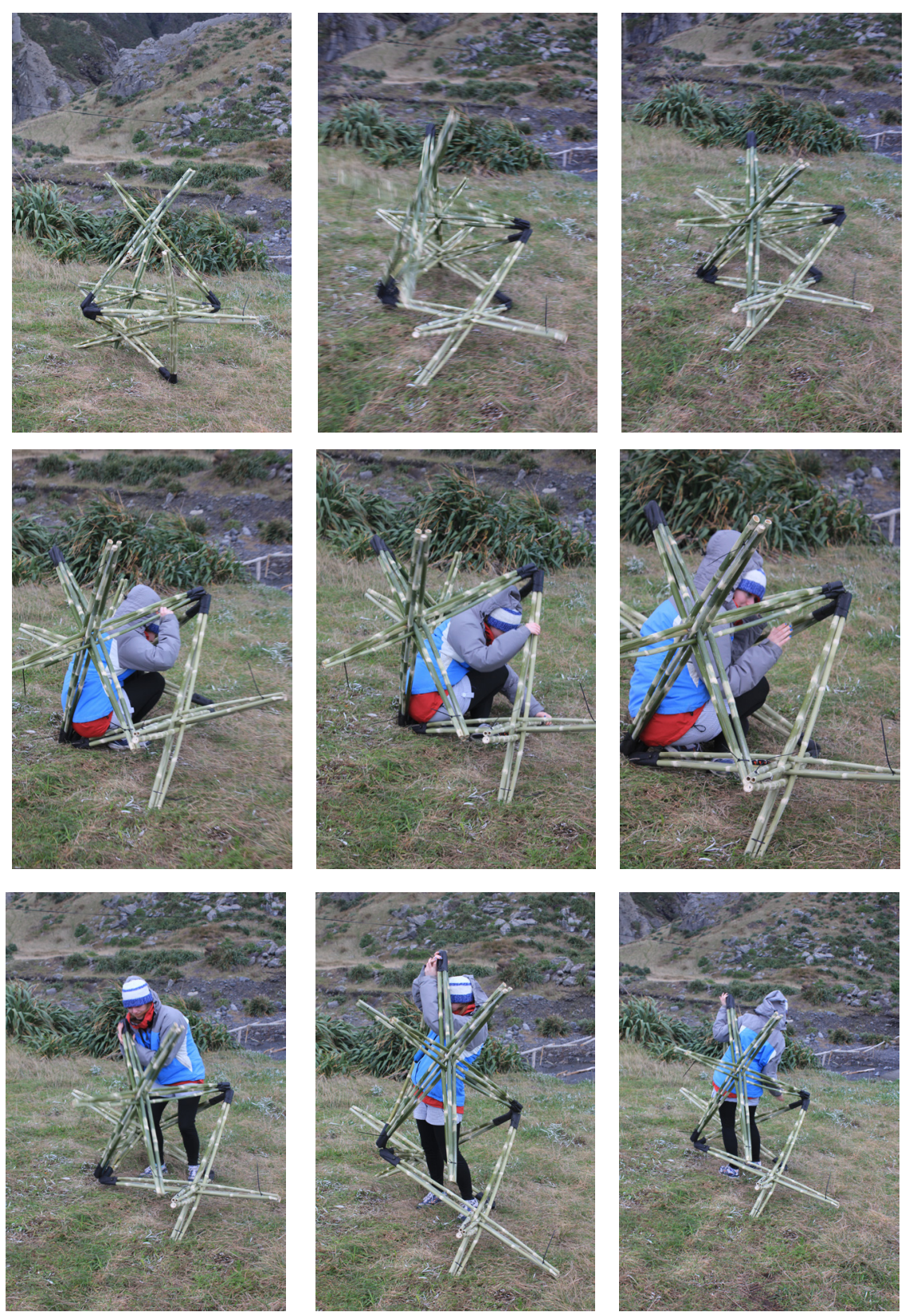

Figure 4.9

1:1 scale stick weaving joints made of bamboo and rubber tubing. Manipulated by person. 

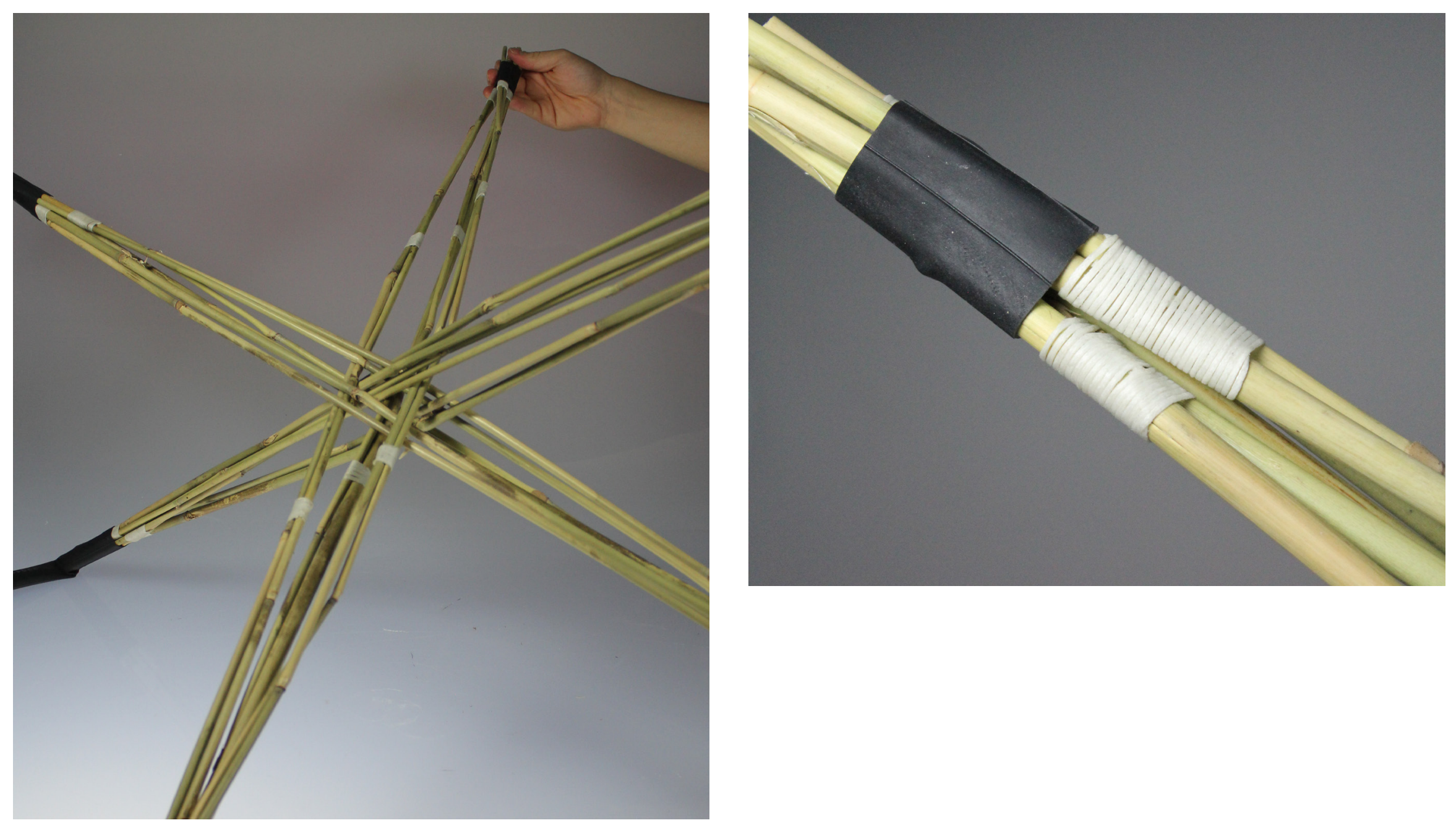

\footnotetext{
Figure 4.10

1:2 Bound stick weaving joints with multiple smaller bamboo
} sticks whipped together. 


\section{Sited Occupation}

In this section I have taken an event based approach to developing program. Initially the idea of taking architecture out to wilderness and wilderness into architecture that was explored in Design iteration $1 \mathrm{~A}$, was revisited. When the weave system was applied to this divergent programme the border between architecture, landscape design and sculpture begins to be blurred. The design varies from inhabited interior spaces to exposed structure that sits amongst the tussocks (refer to Figure 4.11). Separating the programme into individual spaces determined by function to force a constant interaction between participant, interior and exterior. The idea of maximising the programme for its non-static potential was born from this forced movement and became the intention to drive the development of a programmatic layout of spaces. The boulder site was analysed through a series of drawn studies inhabiting the stick weave structure (refer to Figure 4.12)). At a point I moved away from separate spaces for each function and moved to the Concept One design with all programme still occupying the space within the weave but located under one canopy. This change in direction was driven by the ease of watertightness and the influence of the group of pavilion precedents analysed as case studies. The pavilions have a simplicity and clarity that is similar to sculpture as the occupants experience is foregrounded and function often left underdeveloped.
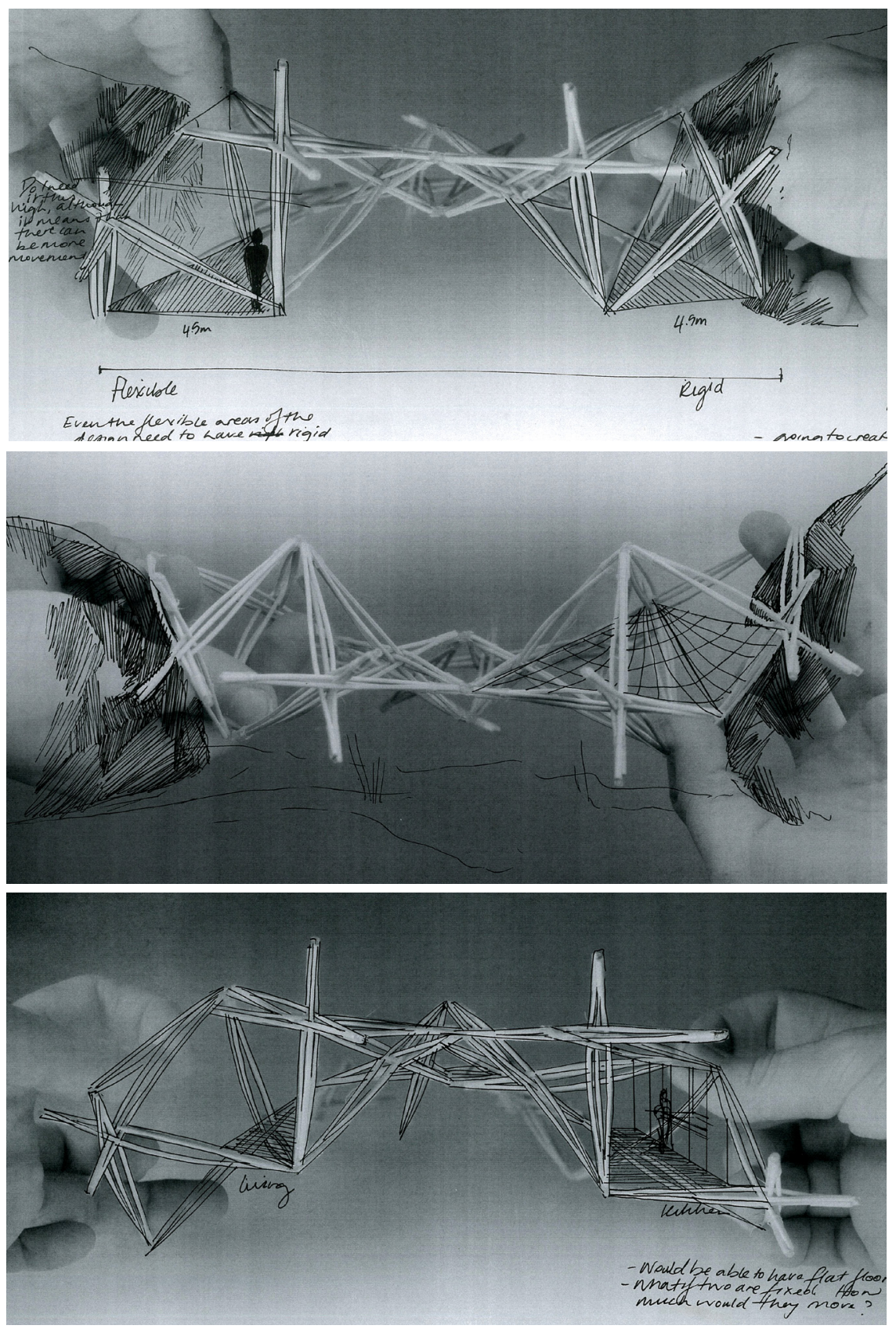

Figure 4.11

Initial drawings that explore how a simple stick weave could be occupied within the structure. Enclosed spaces separate each function with areas of revealed weave that merge with the rough terrain. 


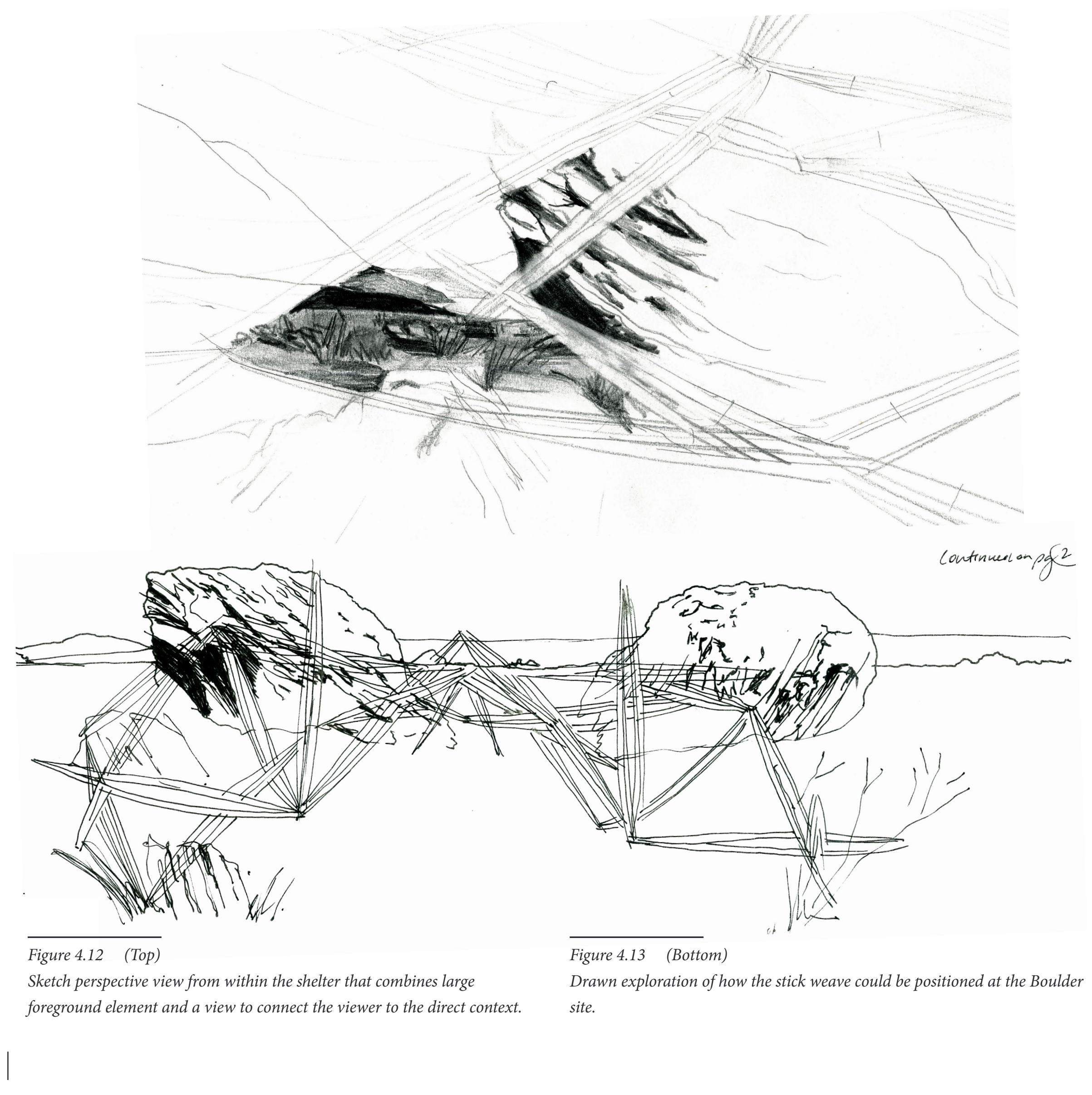




\section{Concept One}

Concept One accumulates the experimentation into physical modelling and membranes, as well as the following movement and programme studies to propose a single weave and canopy shelter sited in between two large boulders. This concept was expressed through sketch plans and modelled physically.

A rough narrative defined movement studies of how participants may travel between the spaces and in turn how this influences an occupant's experience of space. The programme reinforces the natural direction of movement present in the sculpted rocks, splitting key functions such as the kitchen and dining area to create a flow of movement through the centre space. The complete programme including bathrooms and living areas were placed under and within a stick woven canopy that could be manipulated to form sleeping rooms that are soft and sit above the side of the entrance space. The terrain was not altered or covered apart from two stone platforms that form the floors for the living/dining space and kitchen. The kitchen and bathroom utilities were cut into the large boulders to reinforce inhabitation of the architecture and landscape as one. The utilities were reduced to basic functions to maximise central cross-programmed space.

The following drawn occupation studies, plans and images of the Concept One model visually describe this shelter concept, (refer to Figures 4.14-4.18).

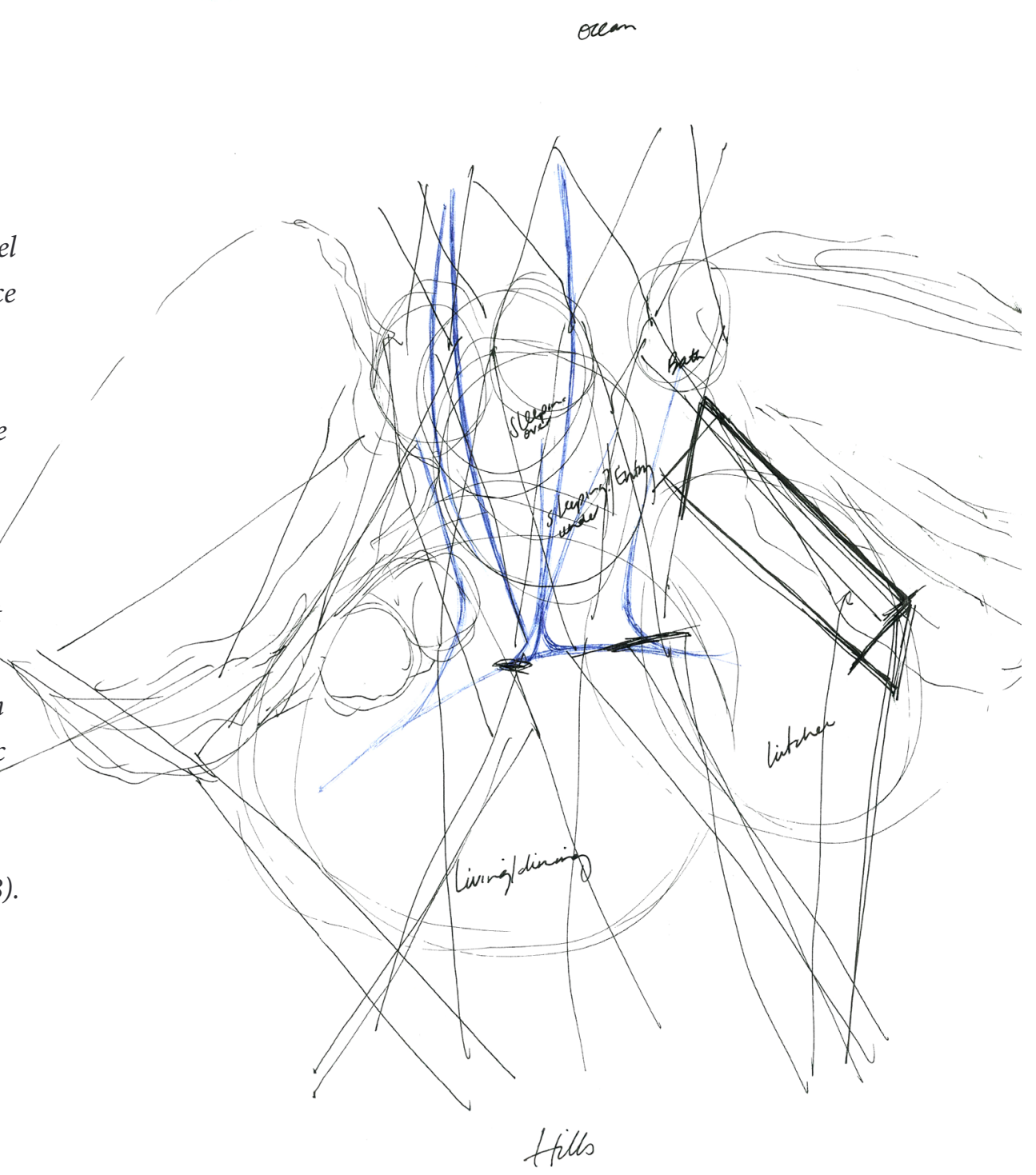

Figure 4.14

Movement study one.

Beginning to explore the potential for maximising movement between each function of the programme. 


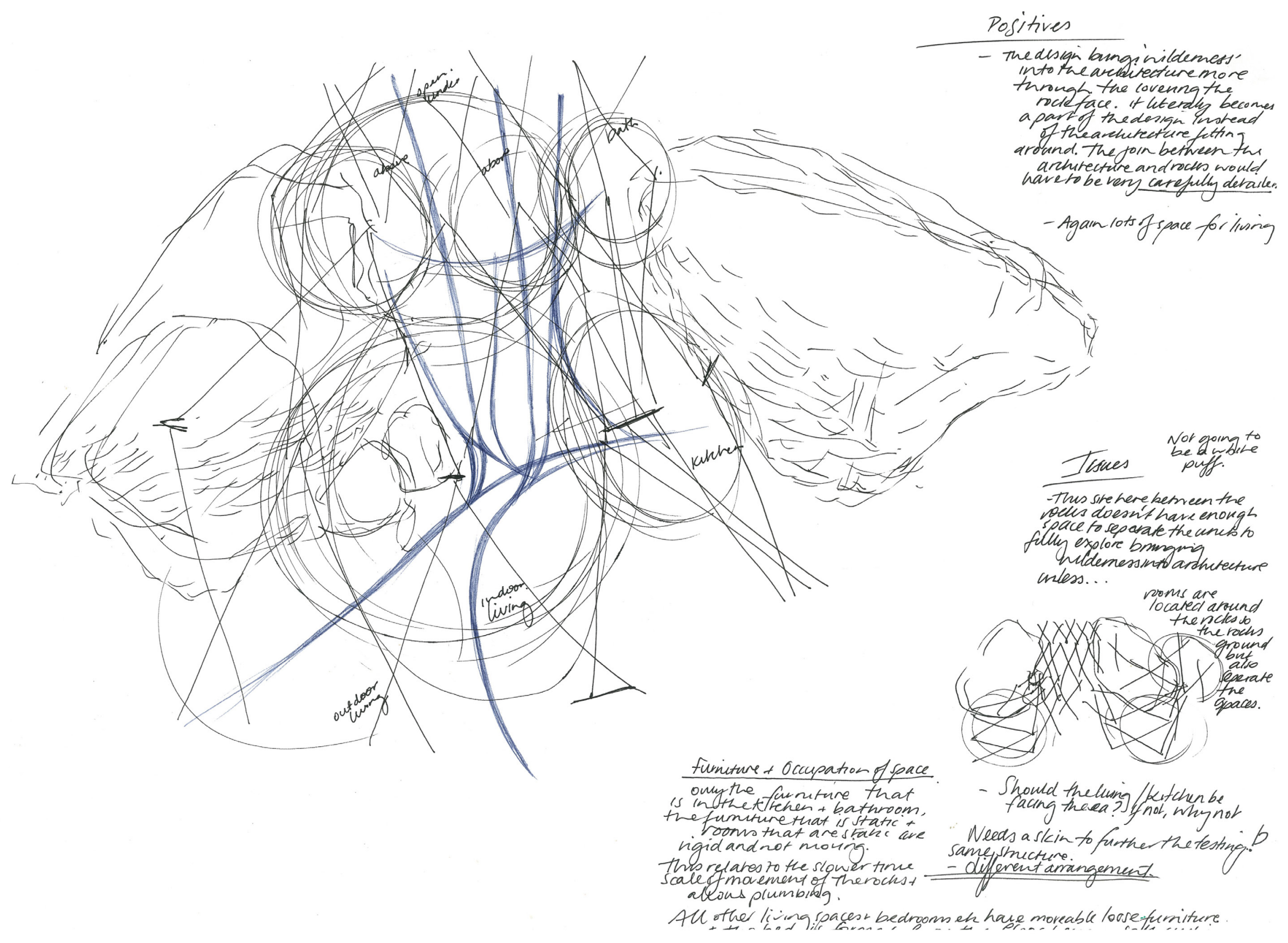

Figure 4.15

Movement study two.

This drawing begins to explore further how openings in the stick weave can emphasise movement paths to enter and exit the shelter.
In the last movement study there was a point in the shelter where the movement stopped. This limits the interaction between interior and exterior and was consequently altered in this study through adding openings. These transformable edges encourage physical, visual and auditory connection to the outdoors. 


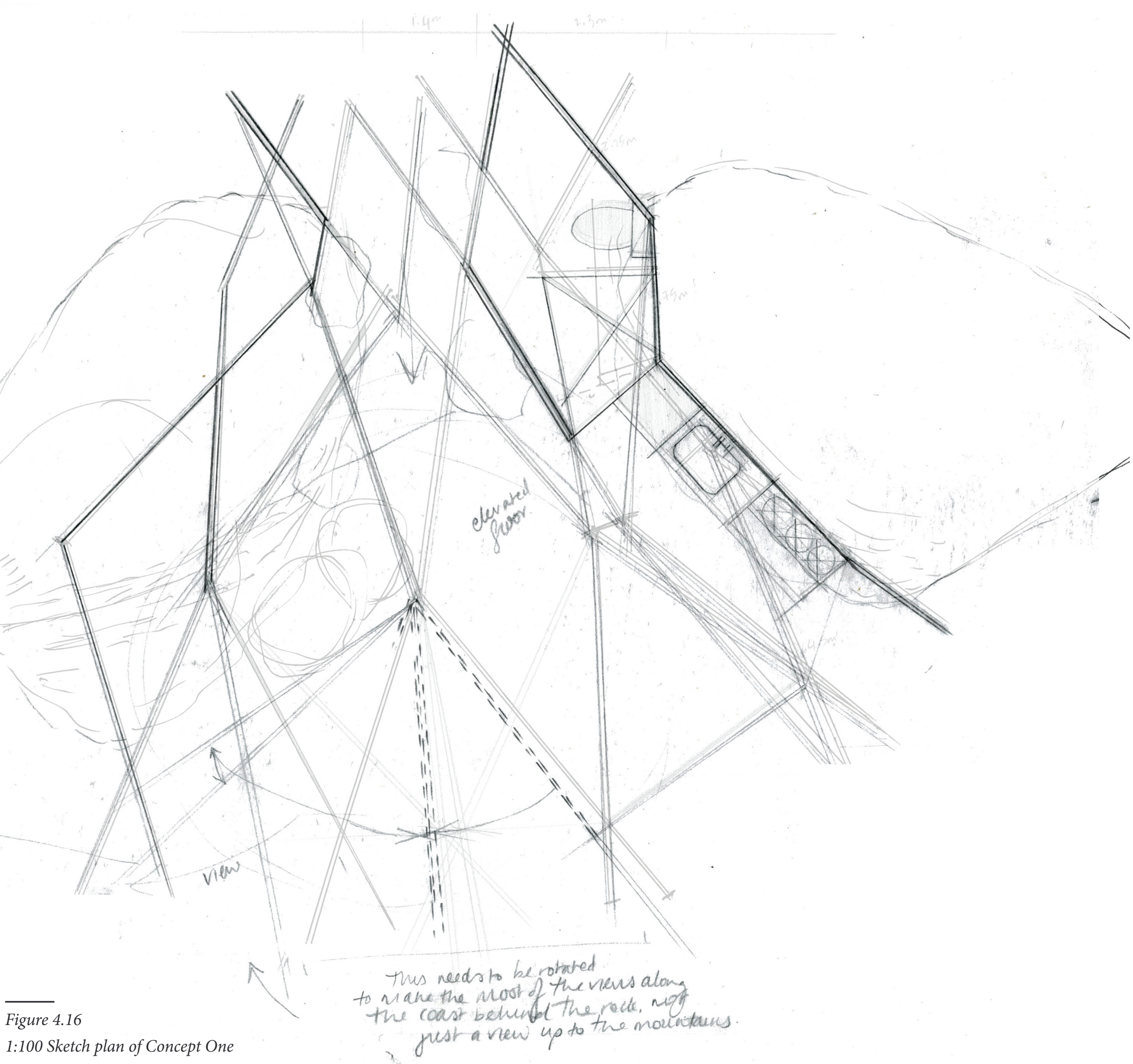



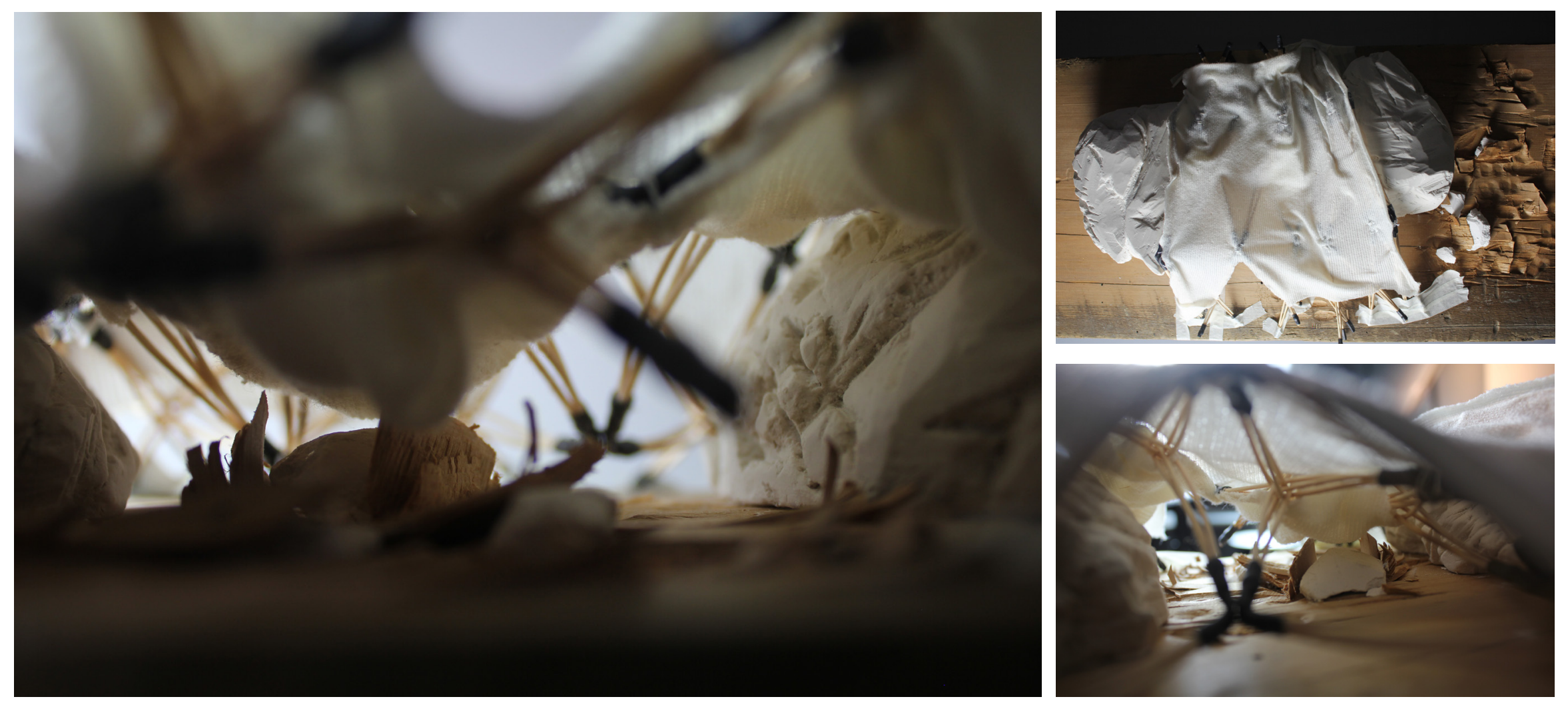

Figure 4.17

Scale model of Concept One, 1:100.

Left: Interior perspective looking from entry to living space
Right Top: Plan view of shelter positioned between scale models of boulders. Right Bottom: Interior perspective looking from living space through to the entrance space. 

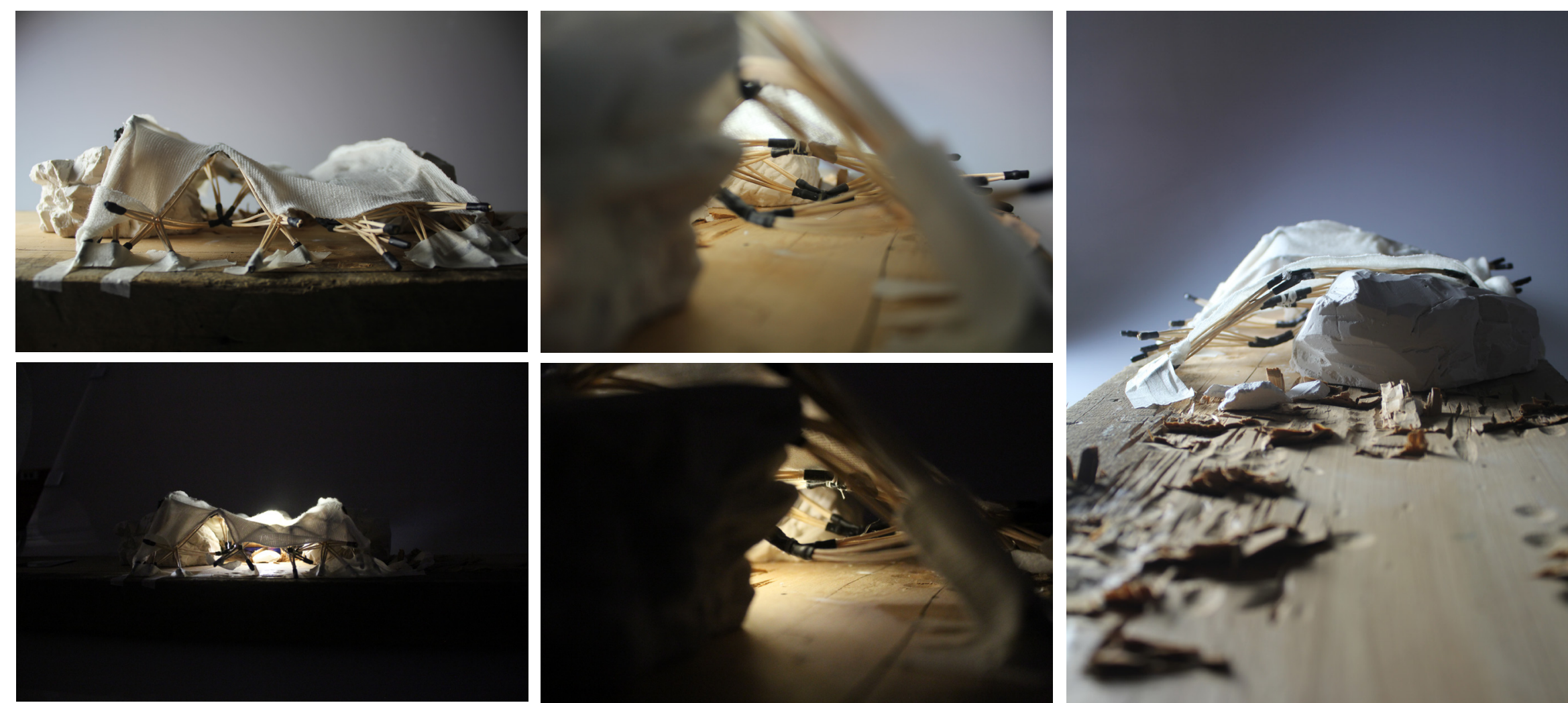

Figure 4.18

A sequence of day and night elevations and perspective views.
This model displays the engagement with the ground plane, although the two stone platforms that would be placed in the kitchen and living area are not modelled. This simple membrane canopy was designed to interact with the rocks on site to create a sense of engagement and ownership of the natural landscape. 
Phase Two Reflection

This phase was grounded in a method of research through making. The process had agency in understanding the relationships between structural and programmatic development and trends within architectural and theoretical fields. A site that embodied the most movement was identified and investigated through a series of photographs, drawings, movement and occupation studies to develop Concept One.

Concept One although designed with the intention of being moveable and fluid ended up being static and unchangeable. The critique highlighted the potential for the design to become more than an occupied pavilion and reinforced the exploration of the initial idea of separating each programme into pods to reinforce the inside outside, architecture-wilderness relationship.

In retrospect, this concept shifts the understanding of occupation of the landscape from a spatially intimate singular architectural space to a wider scale. This resolved concept synthesised the programme and revealed areas that can be cross-programmed to save space. Occupation needs to develop into a more defined narrative through critical engagement with how a person would enter, and inhabit and experience the landscape. This thorough analysis would allow manipulation of the programme, layout, entries and use until inhabitation aligns with the aim of increasing movement, interaction and awareness of wilderness and architecture.

The concept one process exposes areas that need work such as; membrane options, form, siting, transformability and more. Resolved concepts often reveal they are unresolved concepts. The process and period of time that I was developing this sited concept also allowed me to answer important questions such as 'does it need to move'. This re-situated my research in alignment with the affect theories and allowed synthesis of the intention behind the thesis, and to clarify the research question.
The next phase of the design process develops the stick weave further through continued physical modelling and digital parametric modelling to test the extremes of the structure with no material or practical constraints. 


\section{References}

1. Thrift, "Intensities of Feeling," 72.

2. Vo Trong Nghia, "Bamboo Wing," ArchDaily, 2010, http://www.archdaily. com/219880/bamboo-wing-vo-trong-nghia/. 
${ }_{128} \mid$ 


\section{PHASE THREE DEVELOPED DESIGN}

The developed design stage takes the contextual, design-based research and reflection and looks to extend the understanding of how this shelter can directly engage with the landscape through structure and programme. With a focus on the role of analogue and digital design tools for aesthetic, structural and processual development, this chapter seeks to explore two parallel intentions. The first examines how the design process can create an architecture that limits designer control and allows the environment control over the form of the building. The resultant architecture is designed to be removed from the social and cultural influence, as affect is. The architecture is unstructured, uncontrollable and unconceivable to become an interacting part of the environment.

The second intention is to develop through hand drawings, and digital documentation the role of a defined narrative to drive design in this final stage. This phase is an iterative process that oscillates between hand-drawing, physical modelling and the translation into digital form to drive the method to the limits of human control and allow space for environmental interaction.

$-5-$

Investigation

Design Parameter

Design Iteration 3 An Interactive Architecture Form + Structure Programme

Phase Three Reflection 


\section{Design Iteration 3}

\section{Design Parameters}

This thesis treats the practical design constraints of the brief of equal importance as the theoretical inquiry. Architecture operates through built form and the strength of a theoretical inquiry that is grounded by a practical design brief, site considerations and appropriate safety and material constraints surpasses that of a purely theoretical proposition.

Defining practical design parameters is of critical importance to the success of the theoretical proposal for the following reasons. There is a wealth of theoretical analysis of affect but little research through a practical approach. Part of this practical research is embedded in the design process and construction of a building. Secondly, a theoretical proposition where non-static architecture is the mechanism needs to be physically explored in the mode in which it has been conceived. Thirdly and relating to the previous statement, through physical modelling the transformable structural system can be engineered and tested for its functionality and potential for application in other fields. Last of all, the theoretical elements of the thesis still act as the framework for the research question but the prototyping and modelling provide an additional critical ground to draw conclusions.

The site is exposed with extremely windy conditions. The semi-permanent nature of the design means that it can operate as a temporary structure in alliance with the Building Code. This means for wind and earthquake loading the average recurrence interval can be lowered to 1 year as opposed to the 50-year interval for permanent buildings. ${ }^{1}$ This consideration determines the seismic and wind loading strength of the stick weave structure. Despite being grounded within practical design parameters; electrical, water and waste systems are assumed as a part of the shelter but are not a core of my research.

\section{Form + Structure}

One of the main critiques of concept one was the lack of movement in the form. This static canopy was braced to allow inhabitation but this limited the potential for interaction with the surrounding environment. The form from concept one in my opinion did not embody movement physically or aesthetically. Even a static structure can embody movement through its visual form. As a response to this, the stick weave and membrane model from concept one was re-manipulated by hand to investigate the extremes of form as an expression of movement (refer to Figure 5.1). The resultant shapes successfully express movement through the relationship between tensioned membrane and structure, in particular through the stretched folds of material and the visible tension in the sticks. The process of manipulation by hand creates a connection between the designer and the design development that, like the structural modelling in Concept One, explores the generation of affect through process.

A critique of this design process is that this design phase seeks to extend the forms and movement of the shelter past human manipulation to create the potential for environment driven interaction. The method of manipulation in this first step can never recreate this completely uncontrolled interaction, as person-driven manipulation is itself a subject of social and cultural conditioning. A turn to the digital attempts to provides an alternative design process with less physical designer direction and no material constraints. The digital realm has the potential to almost completely remove control so the computer can simulate the random force of wind interacting with the architecture. Digital modelling was also integrated into the design process as parametric modelling saves time, money and resources through fast prefabrication in the construction phase of a building.

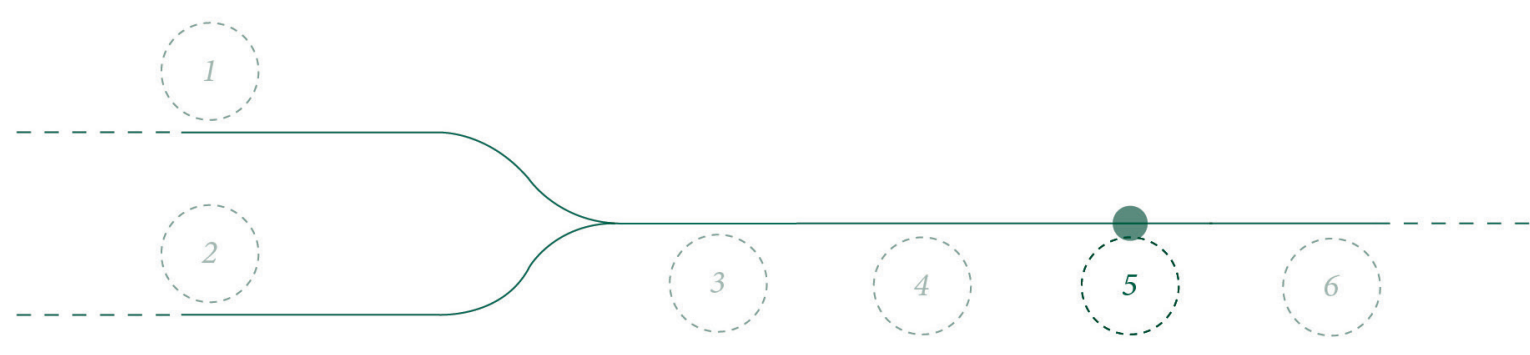



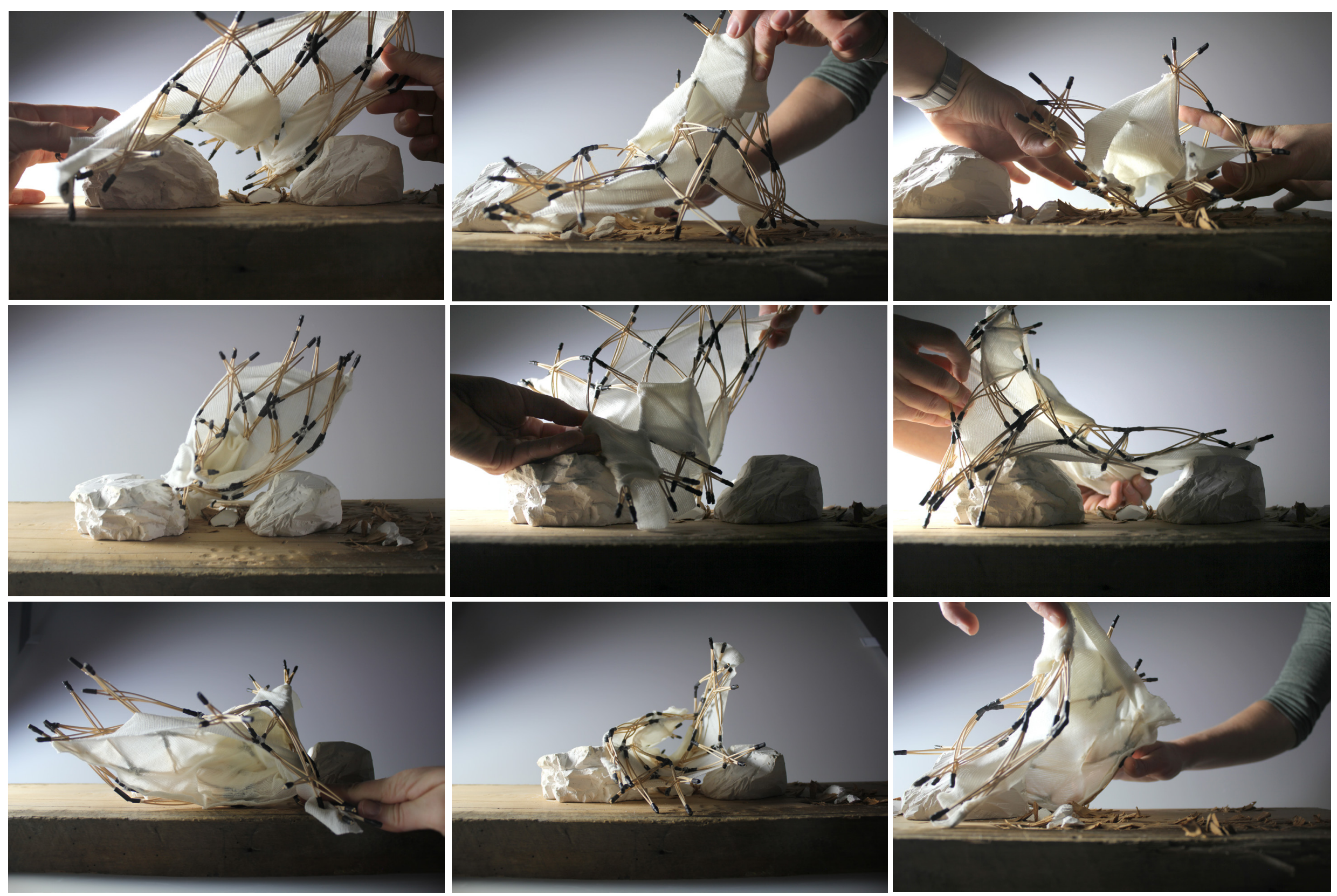

Figure 5.1

Model manipulation to find forms that exhibit the most movement.

One simple stick weave structure can be manipulated into this vast series of forms and movements. Each form expresses the occupiable space differently and it is through the range of movements that these spaces come alive.
It is only through comparison between forms that the nature or feel of the space becomes obvious. For example, in the bottom left image the shelter could be considered open with the large entrance, but in comparison to the bottom right image it appears cosy and intimate. A static form cannot respond to all the needs of the occupants in the way this changeable structure can. 
The digital modelling process began by translating the physical stick-weave into a parametric equivalent. This proved to be extremely difficult involving many iterations as I learnt to model in Rhino, with the Grasshopper visual scripting plug-in. The weave joint was the crux of the issue as even parametric modelling has limitations especially relating to physics principles. As Figure 5.2-5.5 show, the first four digital models allowed movement of individual joints along the $z$ axis but their movement did not influence the rest of the system. I needed to model a solution where the whole system reacted to changes on any point of the model as the physical prototype does. This may be possible for highly skilled parametric designers but within the scope of my thesis I instead made the assumption that the digital weave joint was represented by a point where all sticks cross as shown in Figure 5.6. The form of this final fifth digital model was built as a system that reacts to a series of lines that determine form. This allowed a high amount of control over the shape of the weave so that the physical movement forms could be recreated. The digital modelling process with grasshopper and rhino is explained graphically in Figure 5.7.

The digital process acted as a tool that most successfully removed the material constraints to form curved and stretched members but these experiments were still limited by my intention of translating the physical movement forms (refer to Figures 5.8-5.13).
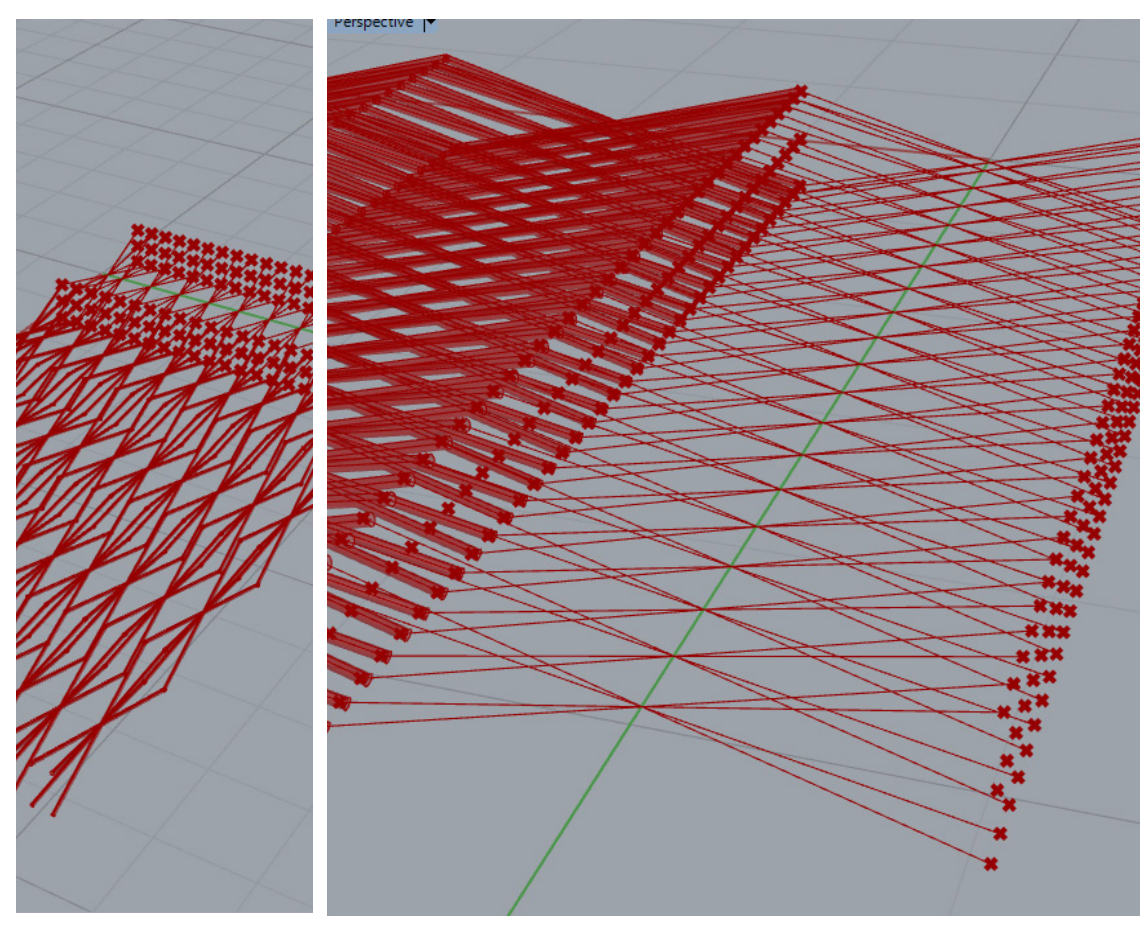

Figure 5.2

Attempt One, translation of physical stick weave into Rhino digital model. This weave looked correct but was not a transformable model and did not allow transformation in the $z$ axis or $x$ axis. 

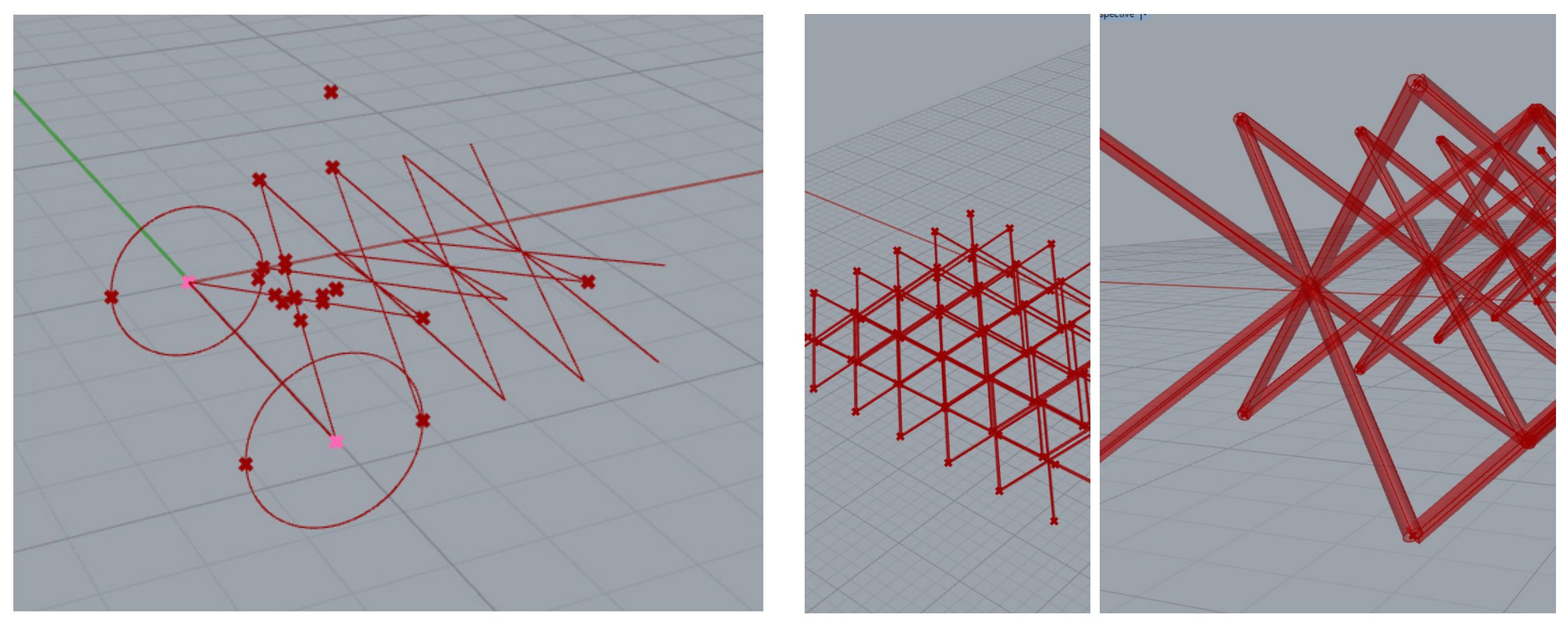

Figure 5.3

Figure 5.4

Attempt Two, translation of physical stick weave into Rhino digital model. This attempt was successful to transform as a whole in the vertical axis but not horizontal. When moved each point of the joint detaches from the next.

Attempt Three, rhino stick weave model.

A grid modelled from two square grids and interlinking lines and pipes. Again no connection between the points when stretched so joints detach. 

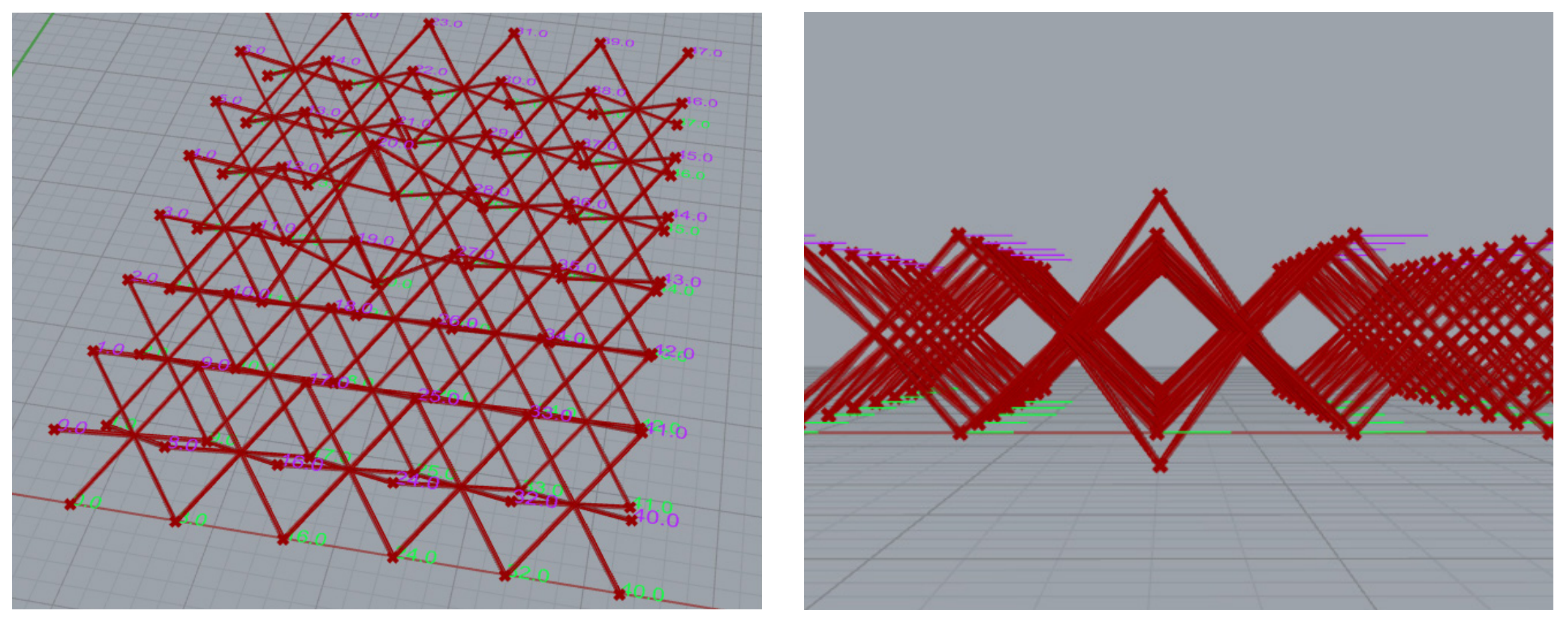

Figure 5.5

Attempt Four; this model aimed to allow controlled movement in each individual joint. This time the grids were labelled and list item components were inserted into grasshopper to identify points, and manipulate them.

Despite this the model was not very robust and when altered for improvements quickly went into disarray. Also the movement of points did not affect the rest of the system which is critical to recreate the weave movement from the physical model. 


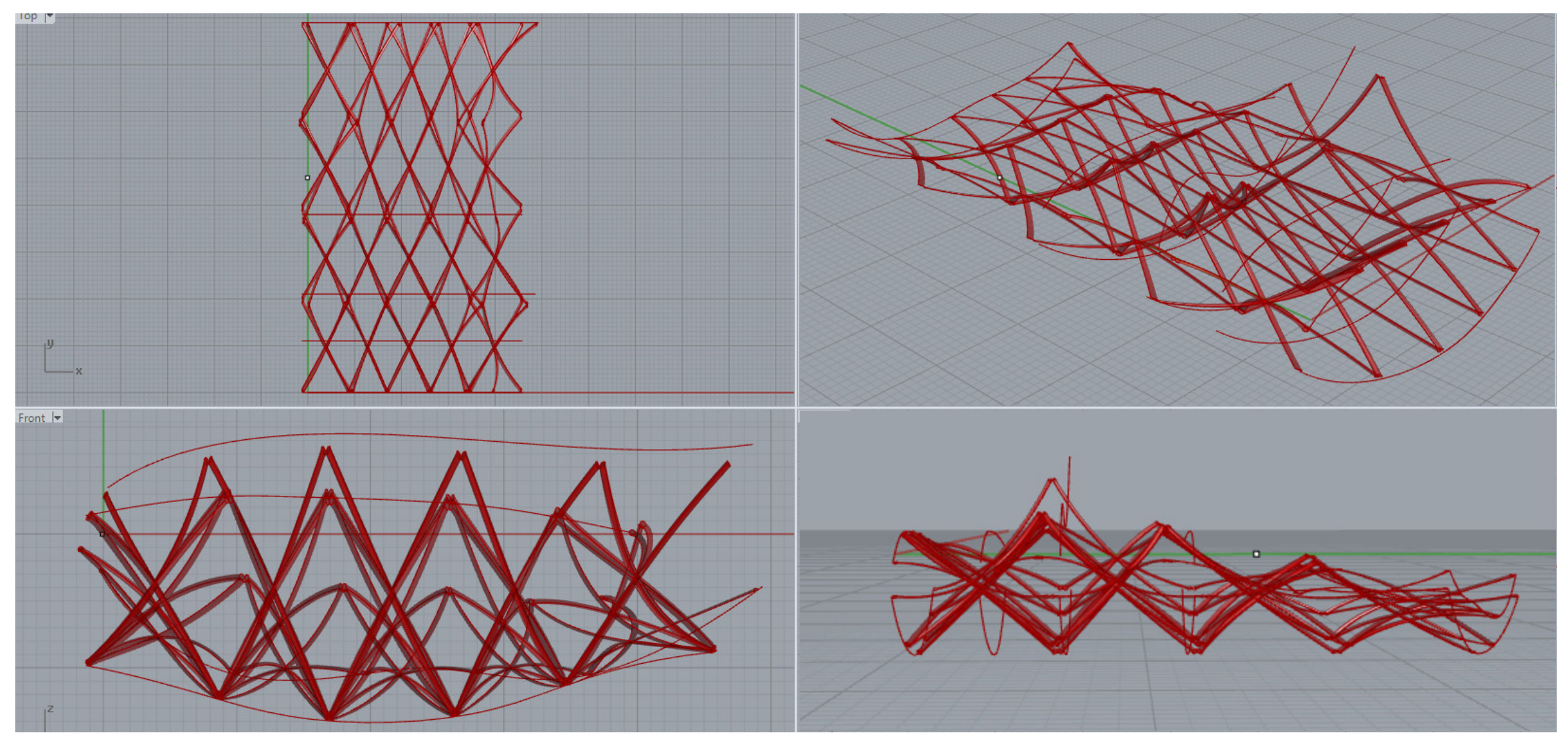

\section{Figure 5.6}

Attempt Five, the weave is formed by distorted square cubes between upper and lower grids that are controlled by the placement of a series of lines. This resulted in a complete system that interacts and reacts to changes in the position of the lines. Any form and space is able to be modelled by adding more lines to increase the detail.
The main issue with this model is that there is a lack of control of individual joints as lines did not correspond to joins and in the $x$ axis the joints are evenly spaces along the lines. Note in the bottom right image the control lines are located at different positions to the cross joins, and in the top left image the even spacing of joints. This means a different method of form generation is required to recreate the physically modelled forms. 


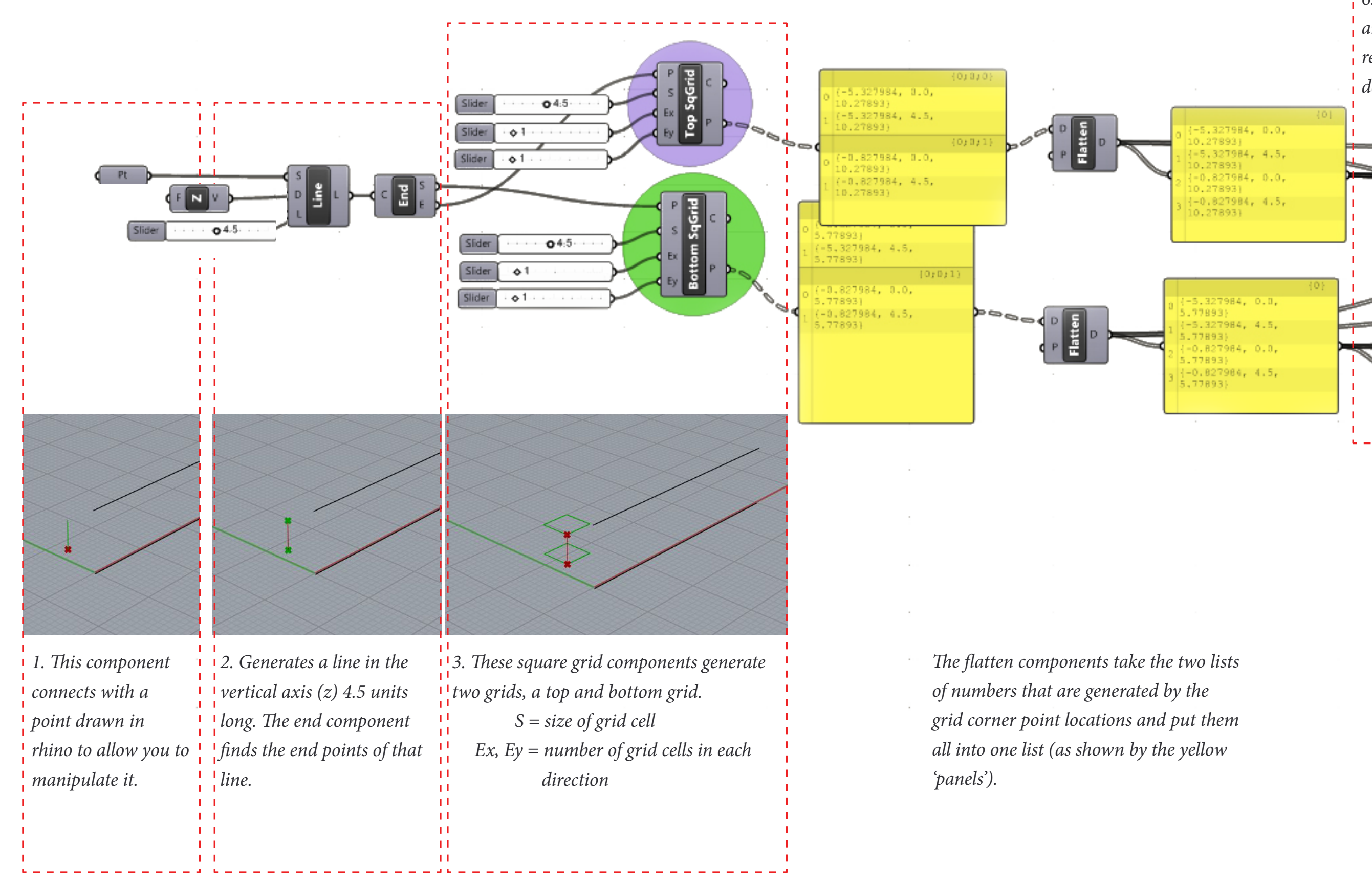

\section{Figure 5.7}

This diagram is page one of two of a sequential explanation of how the grasshopper programming generates the successful 'Attempt Five' stick weave model. The following page of programming connects to the top right component on this page. 


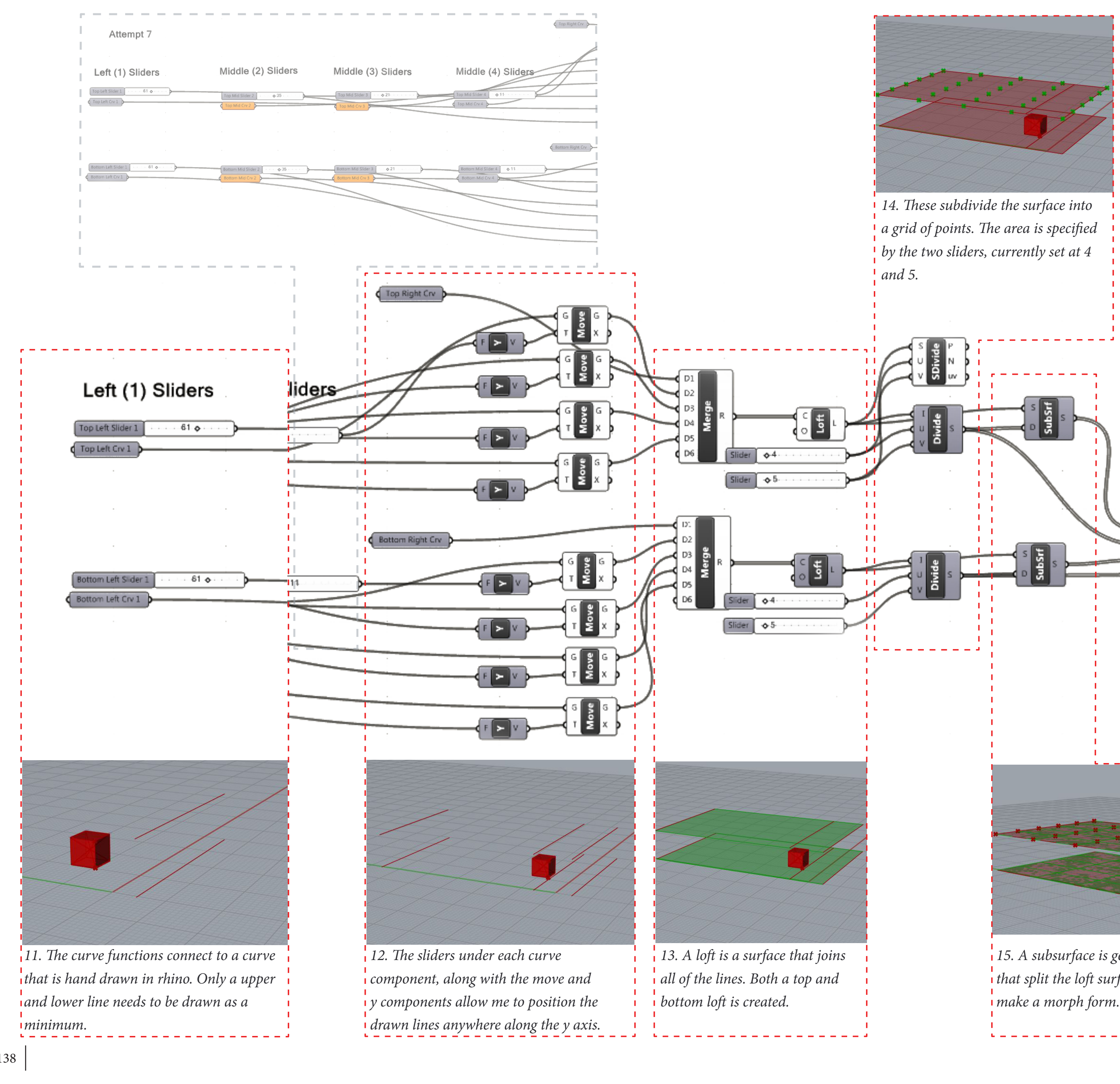




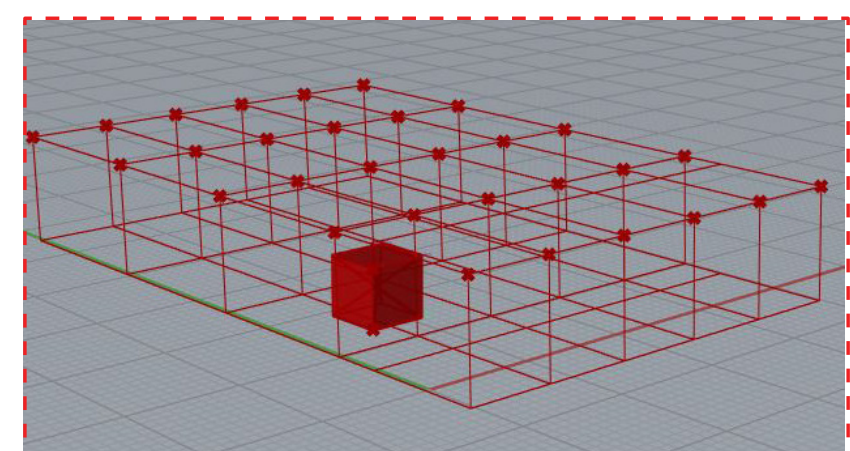

16. This step creates boxes that connect the two grid surfaces. A blendbox lets you create 'twisted boxes' between two surfaces so even when the lines dictate a more unusual shape this same grid is held and the volume and shape of the boxes can change, yet they remain connected.

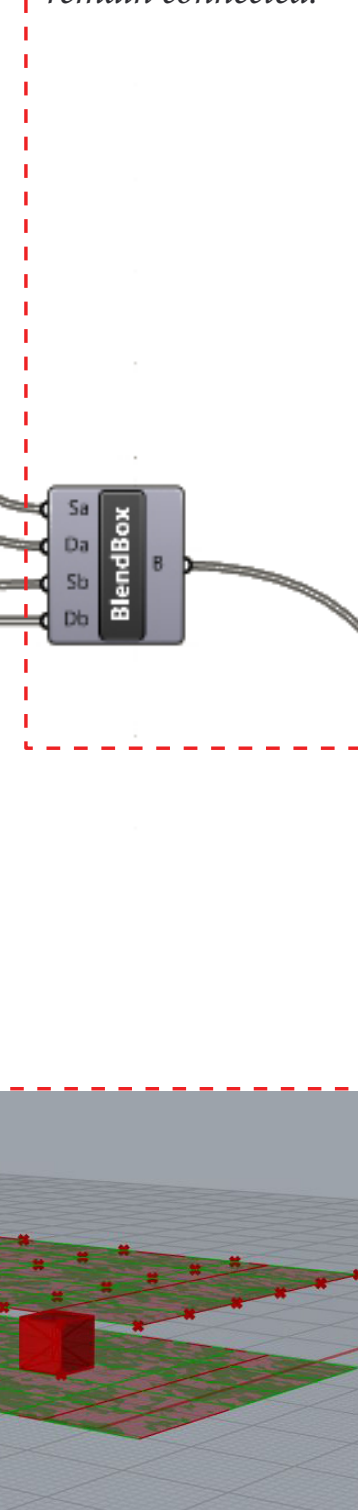

nerated by the grids

17. The morph component places an object within each of the boxes. In this case it is the cross ace. This is necessary to $1:$ joint to create a weave network. The crucial different with this digital model in comparison to I the previous is that it acts as a manipulatable whole, when one joint is moved it influences the 1 rest of the connected joints.

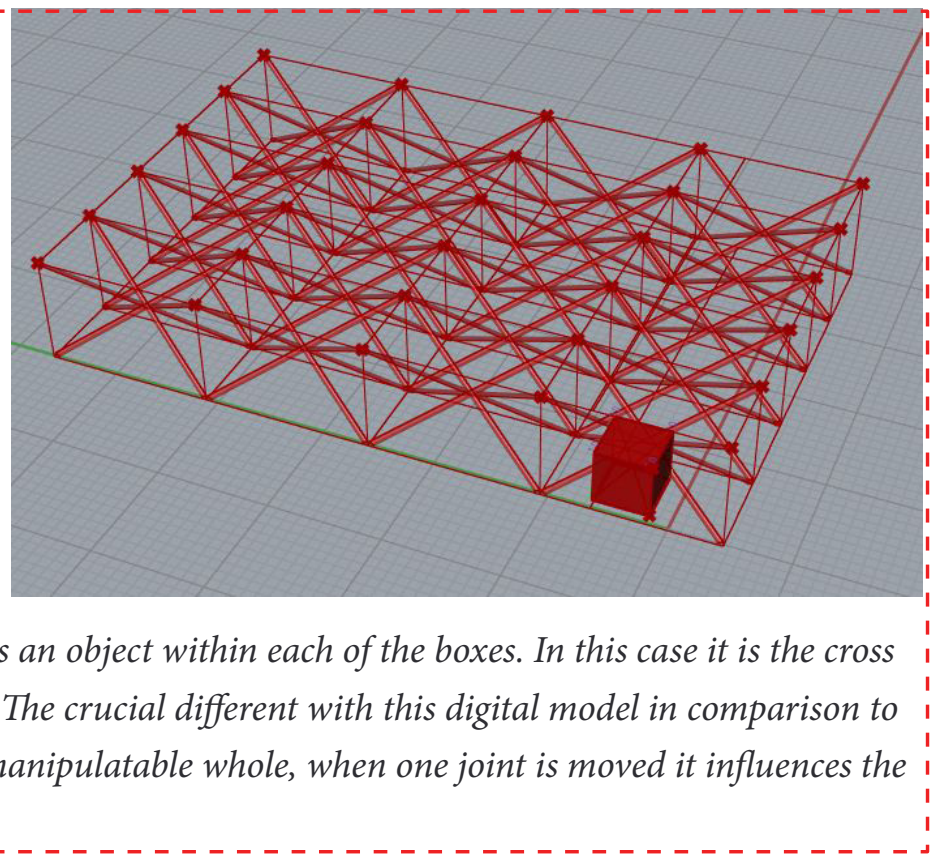

This image displays the possible forms that can be created by this model, and the relationship between each joint. 

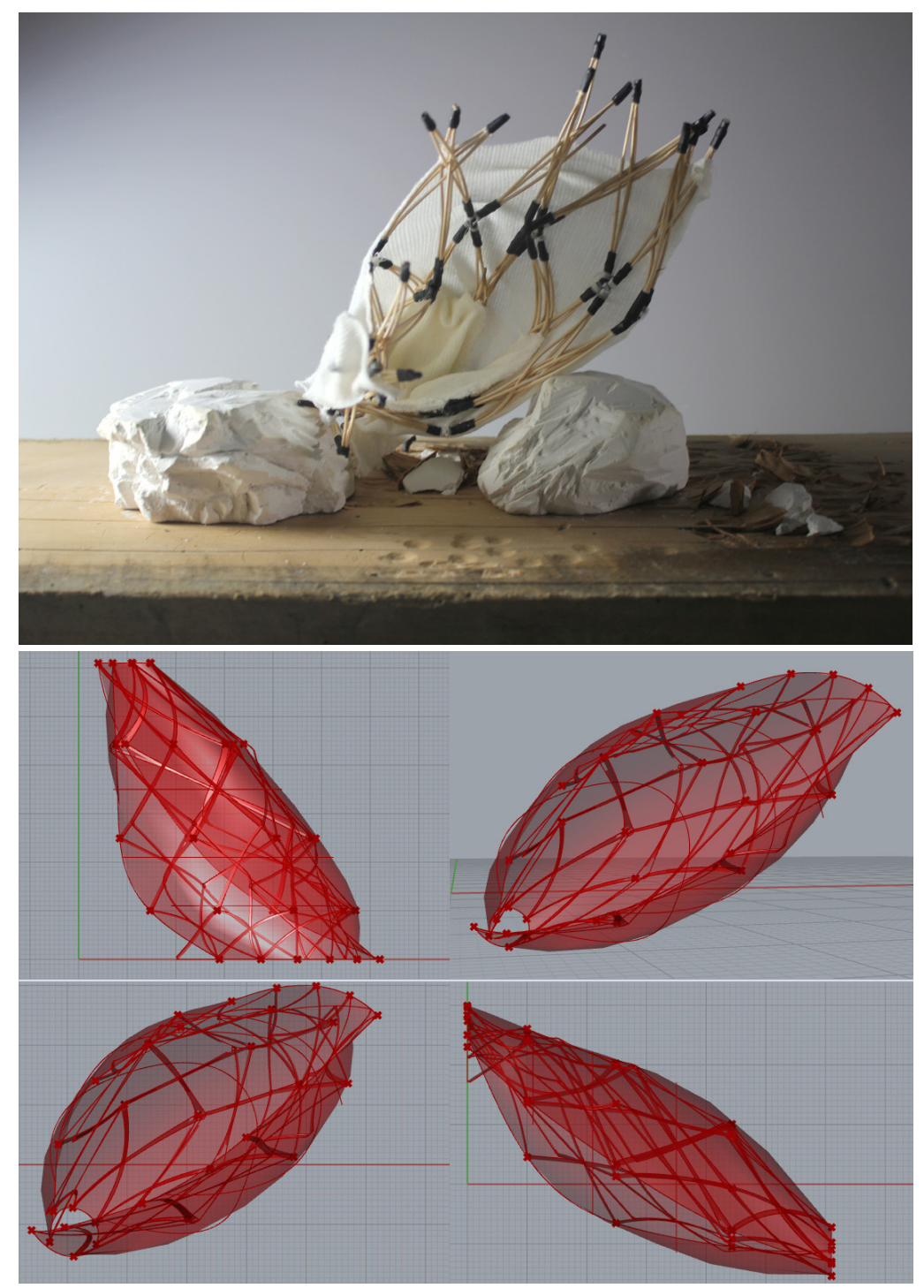

Figure 5.8

Digital model one; recreating the fluid physical forms through digital modelling. The intention is to compare the differences between physical and digital modelling to try and maximise the potential for movement and fluid forms from the structure.
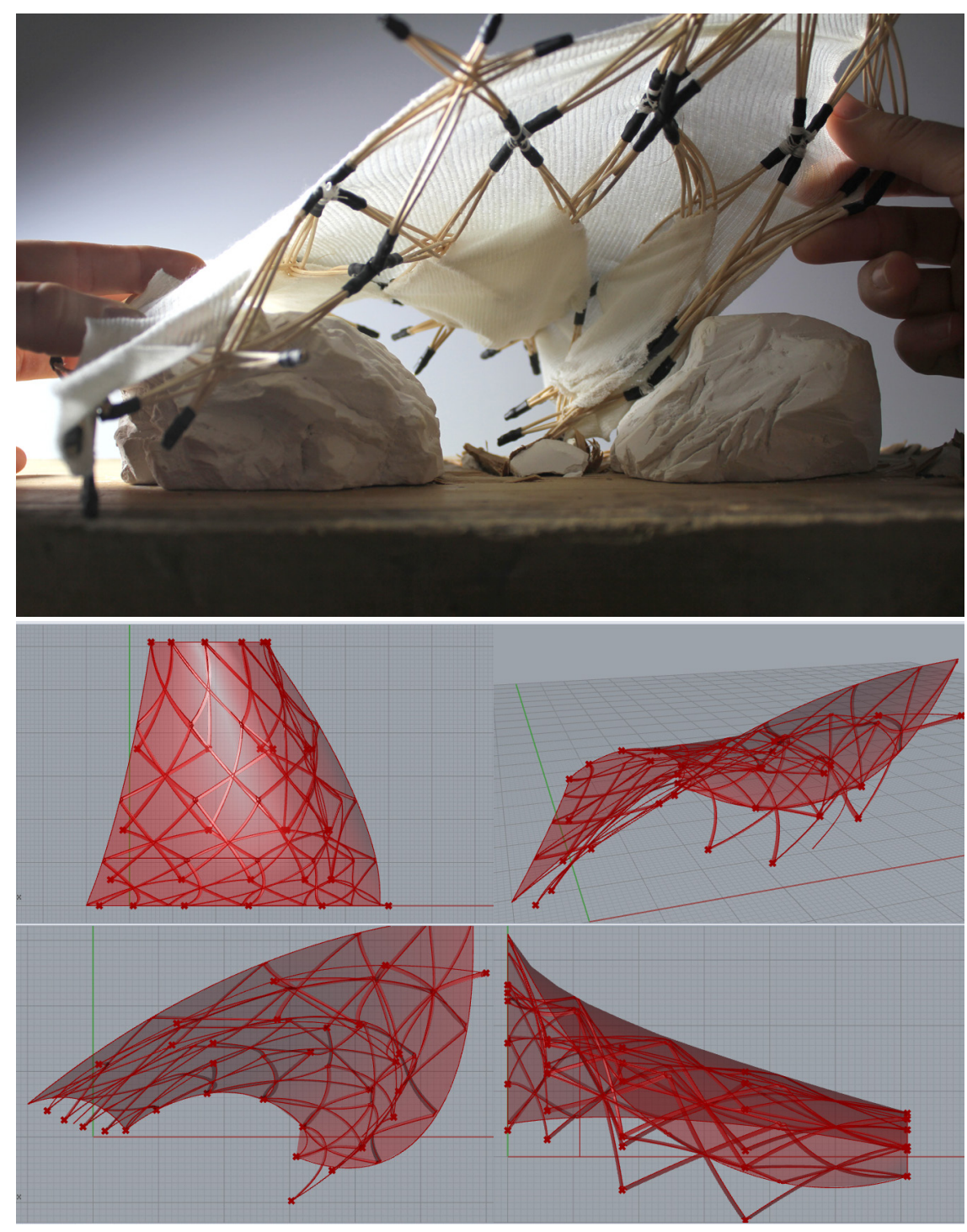

Figure 5.9

Digital model two. The digital models have no material constraints and are based around the even distribution of the points along each axis. Notice the curved stick members. 

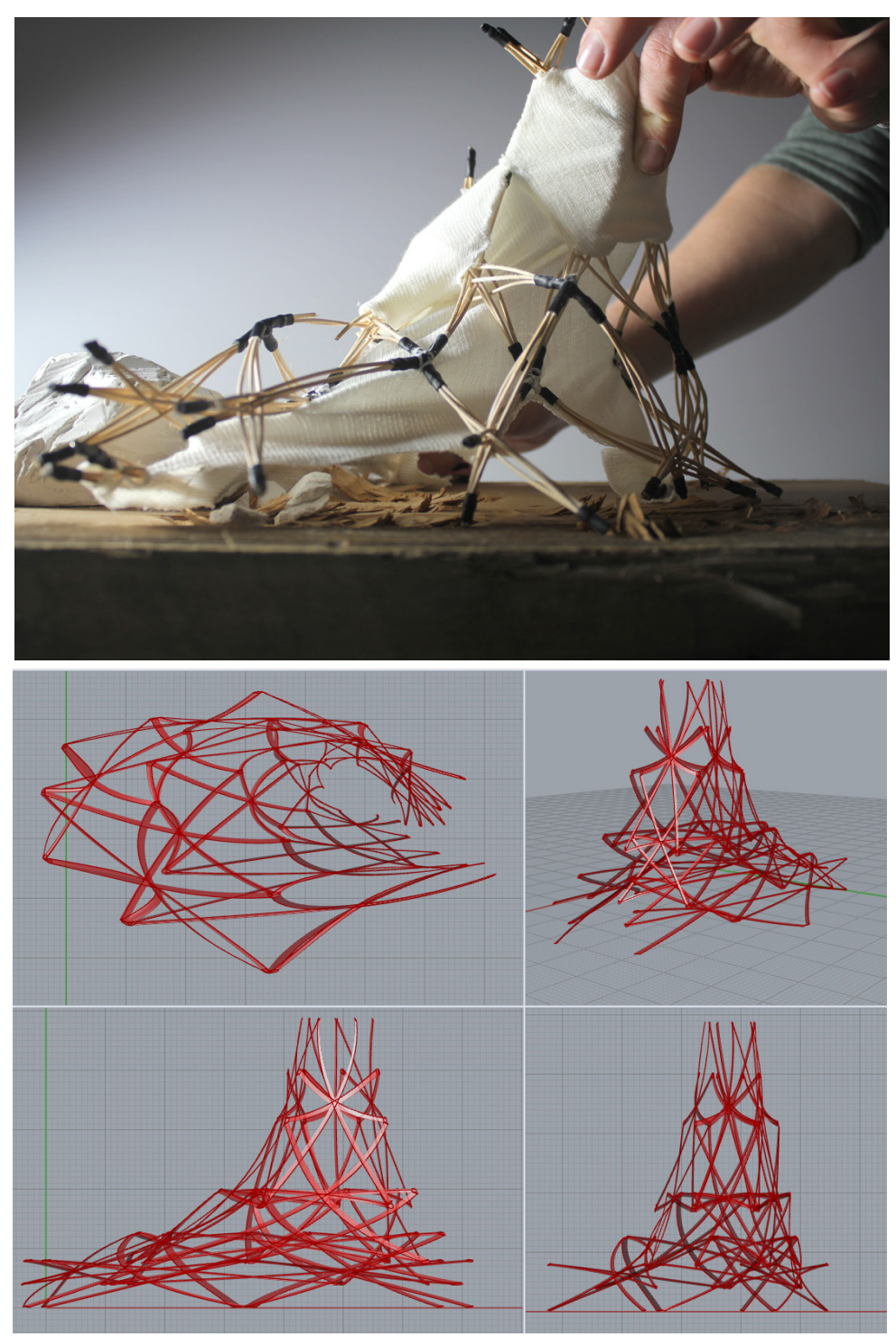

Figure 5.10

Digital model three. The closer geometric arrangement of point connections up the vertical part of this form is obvious in the digital model. This creates a seemingly over-complex and distorted structure to recreate the same form.
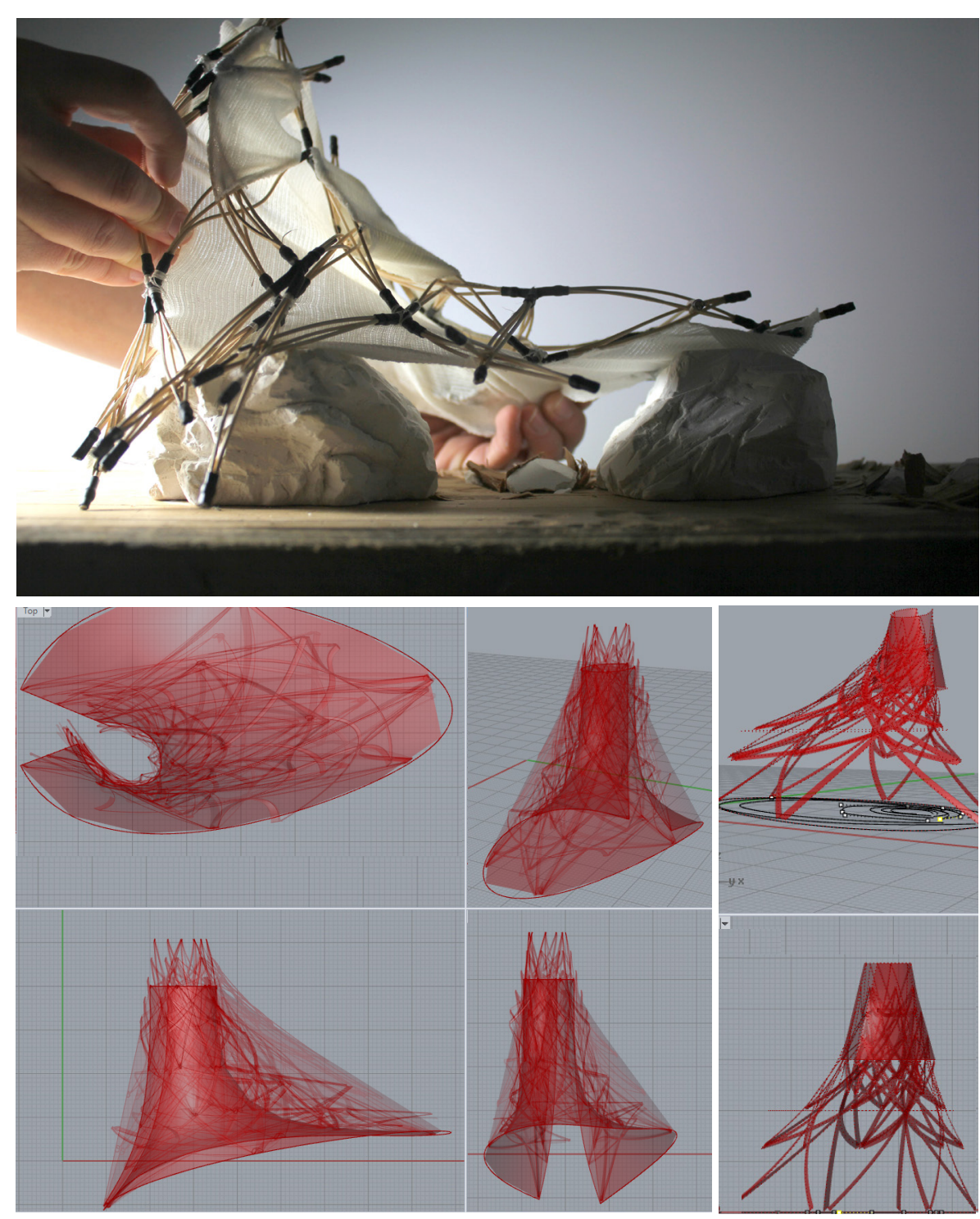

Figure 5.11

Digital model four, very similar to the previous form. Through a process of random alterations to the sliders in grasshopper accidental designs were the most interesting as they challenged the limits of the physical stick-weave that could be limiting the design. (bottom right model, nested aesthetic) 

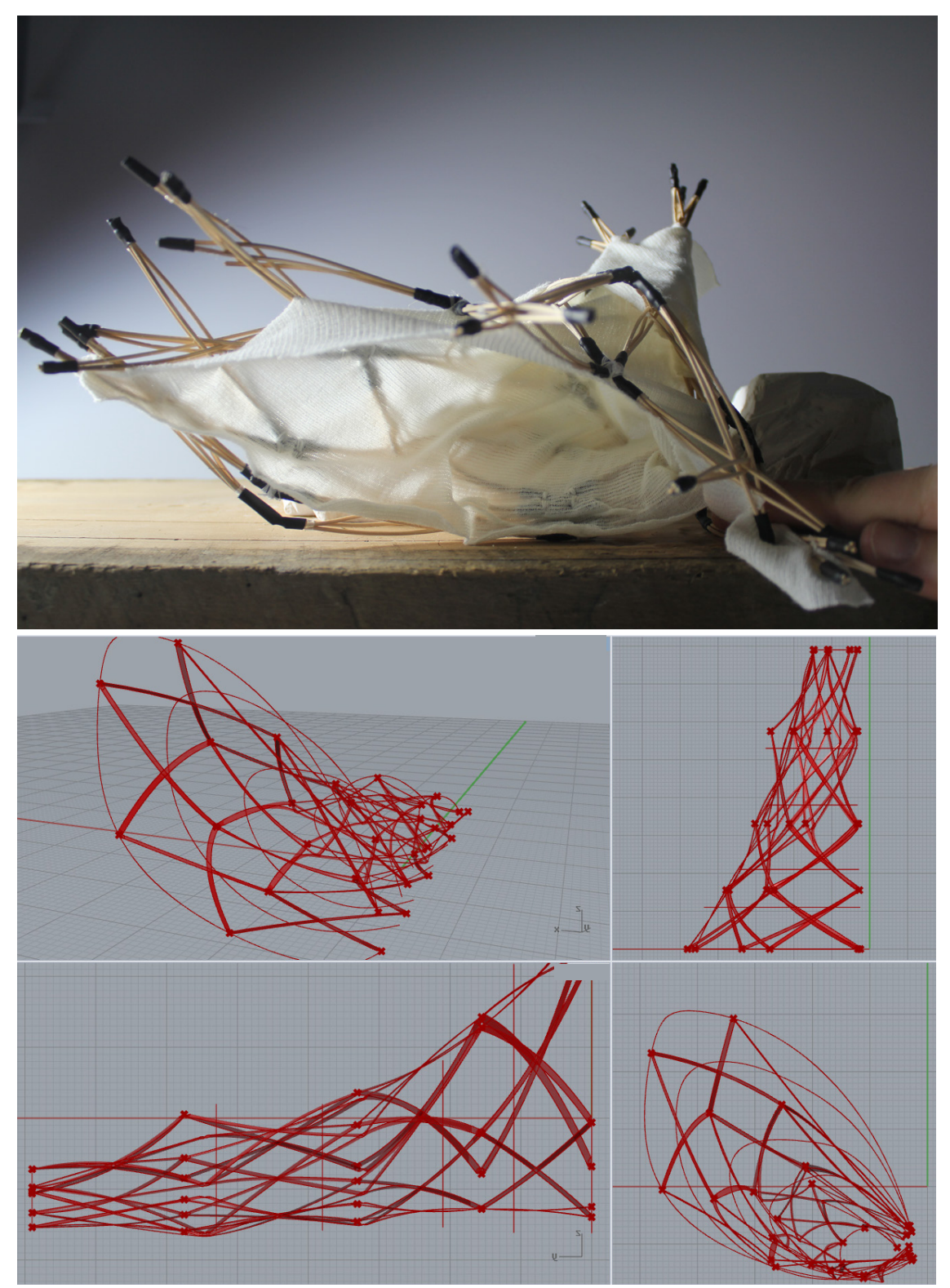

Figure 5.12

Digital model five. These last two forms were the hardest to model digitally as I was not able to determine where the ends of the joints lie and this is the strongest movement indicator in the physical models.
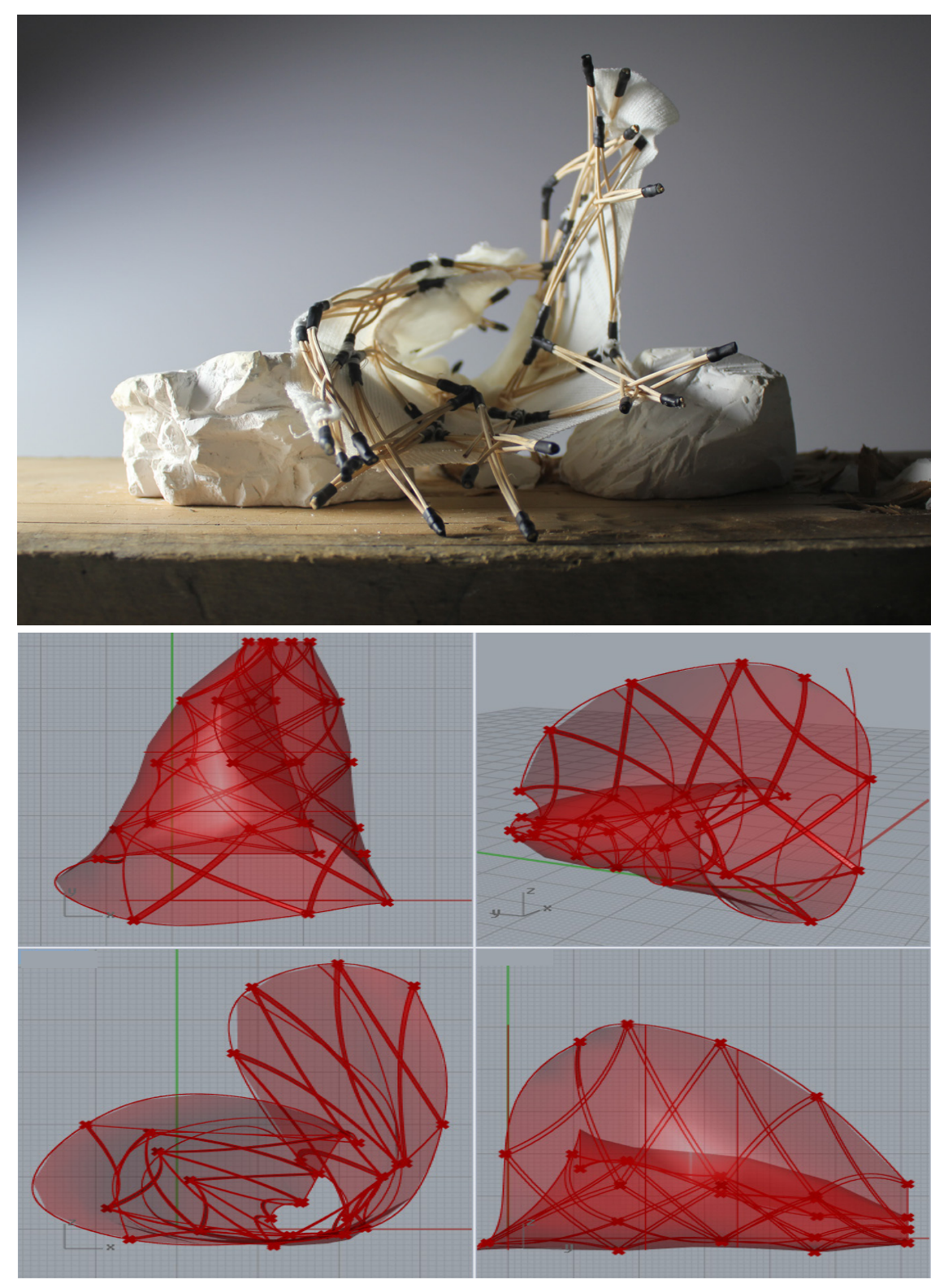

Figure 5.13

Digital model six.

There is a lack of visible movement with the digital models. When a loft is added (the red fabric-like layer) there is a greater visible understanding of movement. 
The parametric design process was pushed further into the removing of control by creating a series of parameters so that the computer can run through all mathematically possible form options. The computer therefore simulates the randomness of wind action upon the architecture, (refer to Figure 5.14).

The important realisation at this stage of the design was that there has to be a level of control by the designer to enable occupation and successful engagement with the public. The designer influence was minimised as much as possible to: necessary fixed points of contact between the structure and the ground, functionality of each shelter, and the relationship between environmental influence and participant influence. This control is needed to most effectively heighten the awareness of the relationships between architecture, environment and people.
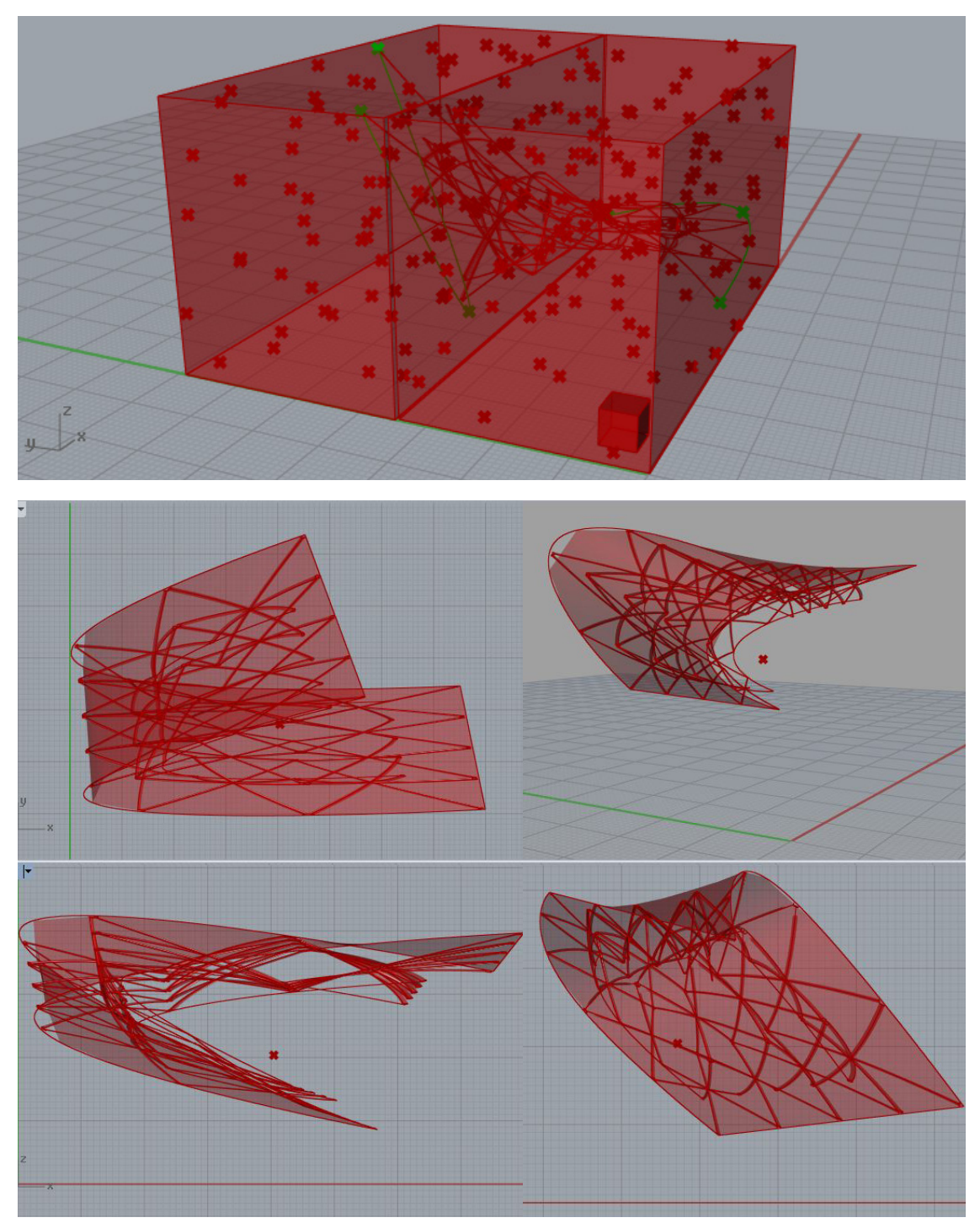

Figure 5.14

An example of random digitally generated forms using Rhino and Grasshopper. The lower image shows how the random lines were generated. A box was randomly filled with points, three of these were connected with a curve to generate the lines to create the form. 


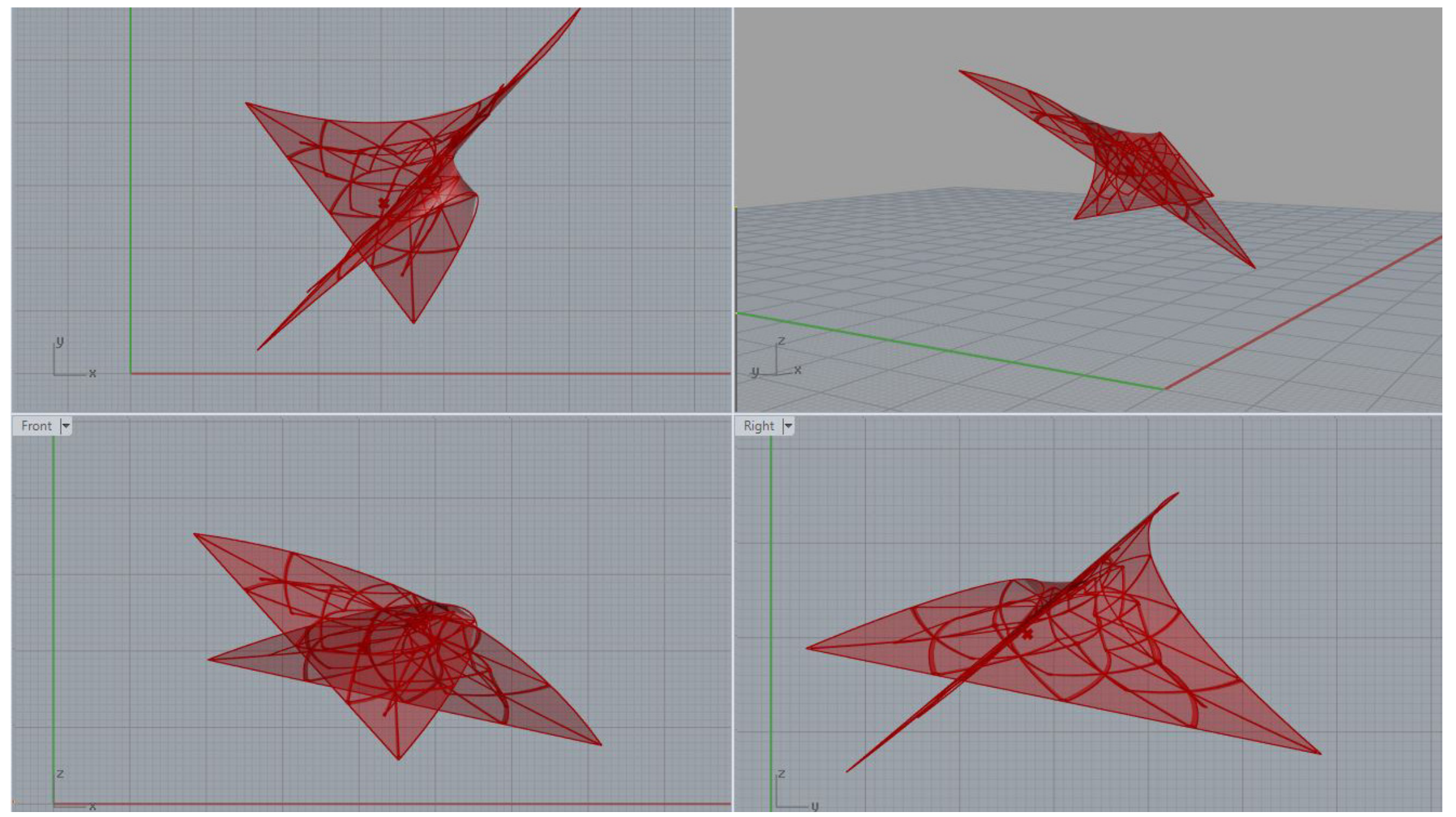

Figure 5.15

Video stop motion sequence showing 100 different options generated randomly through the digital modelling process. In this example only one of the two lines was changed so one line remains constant.
These two videos were included as it is essential to reinforce that the digital and analogue forms presented visually in this thesis are representations of endless possible forms that could be created by people or atmospheric conditions. 


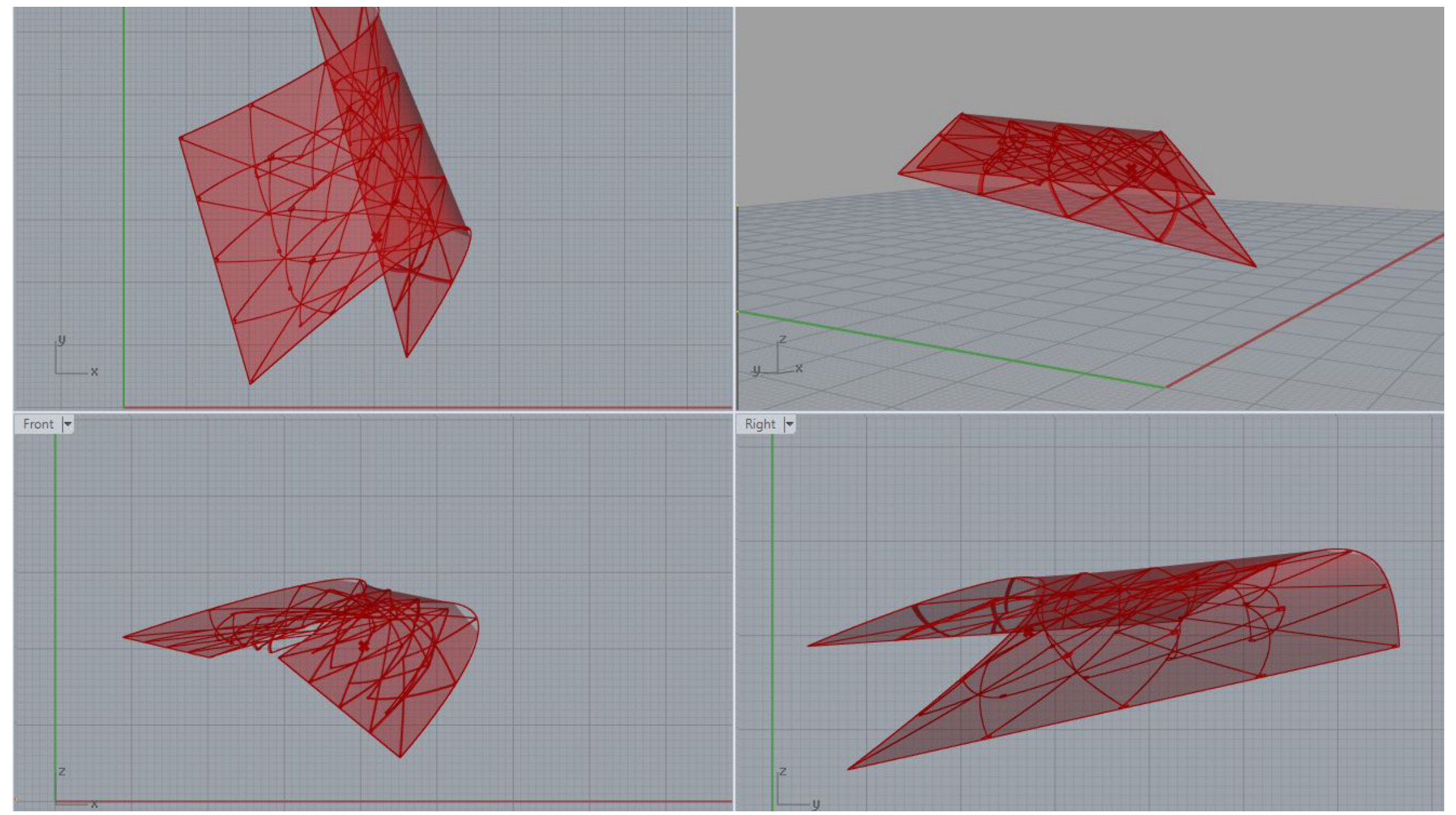

Figure 5.16

Second example of random digital form generation. Both of these sequences show the success of this transformable structure. In this example both of the lines that determine the form were randomly changed. 
Throughout the design development phase physical modelling and rapid prototyping was undertaken to resolve areas of the design in detail such as; further options that enhance inhabitation and floor-weave connections as is shown in Figures 5.17-5.20). Despite this, due to the complexity of the stick weave structural system complete resolution of the joints is outside the scope of this thesis. Should further development of this stick weave system have occurred, a process of detailing structural controls would be undertaken to filter the interaction between wind and the shelter. Working with engineers, the connection joints and curvature of the stick members could be then tested until breaking point to determine the amount of flexibility in the sliding action of the weave joint. These controls are necessary in a built design to firstly make the building safe, and more importantly to refocus on the manipulation of space and time through slow motion.

The proposed joint detailing that would be break tested (should further development occur) is outlined in the following programme section. These details sit alongside a complete description and discussion of the final shelter design.
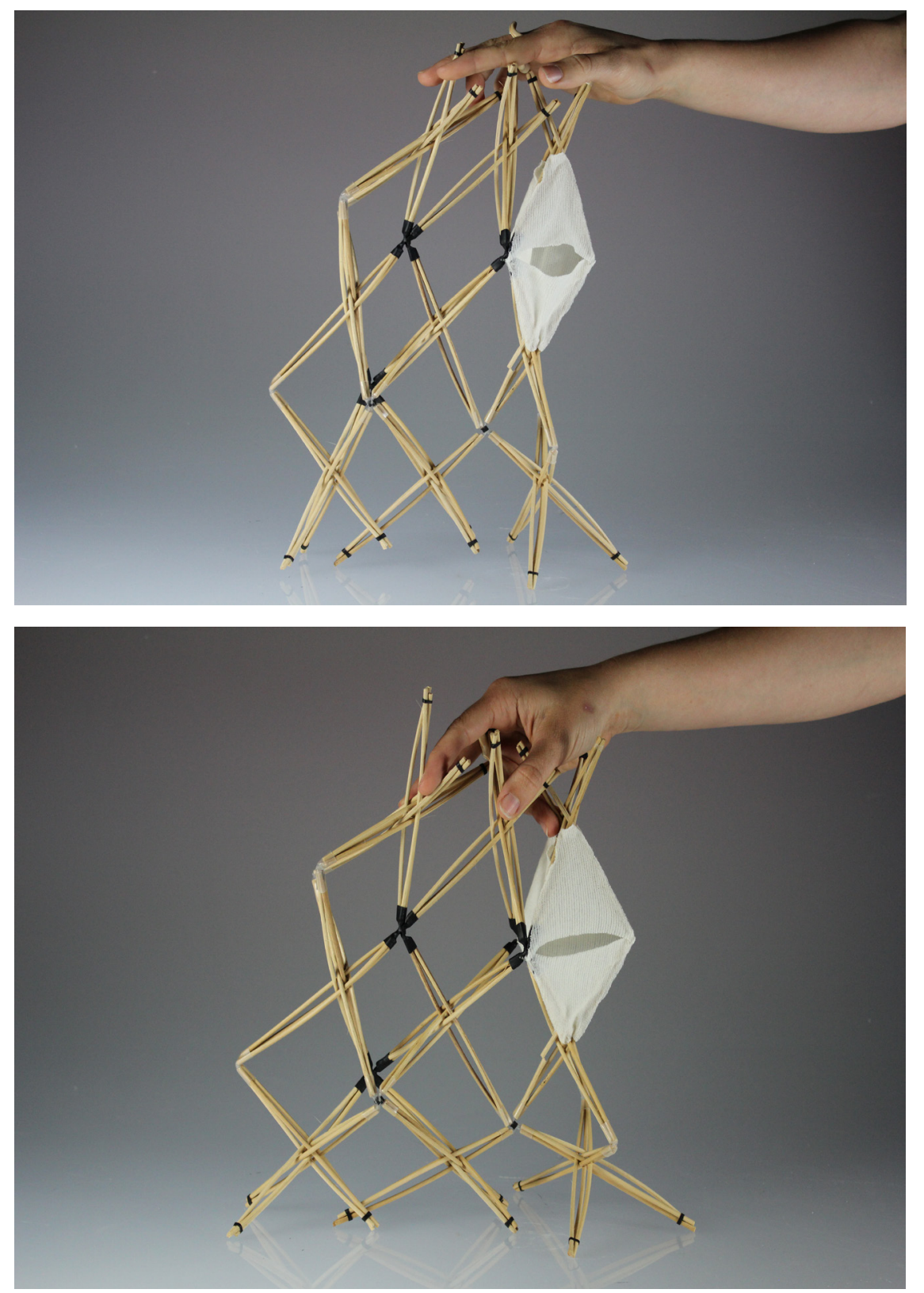

Figure 5.17

This model was constructed to explore possibilities for other uses of the weave such as storage. A soft fabric was attached to each member which had enough stretch to allow complete deformation. 

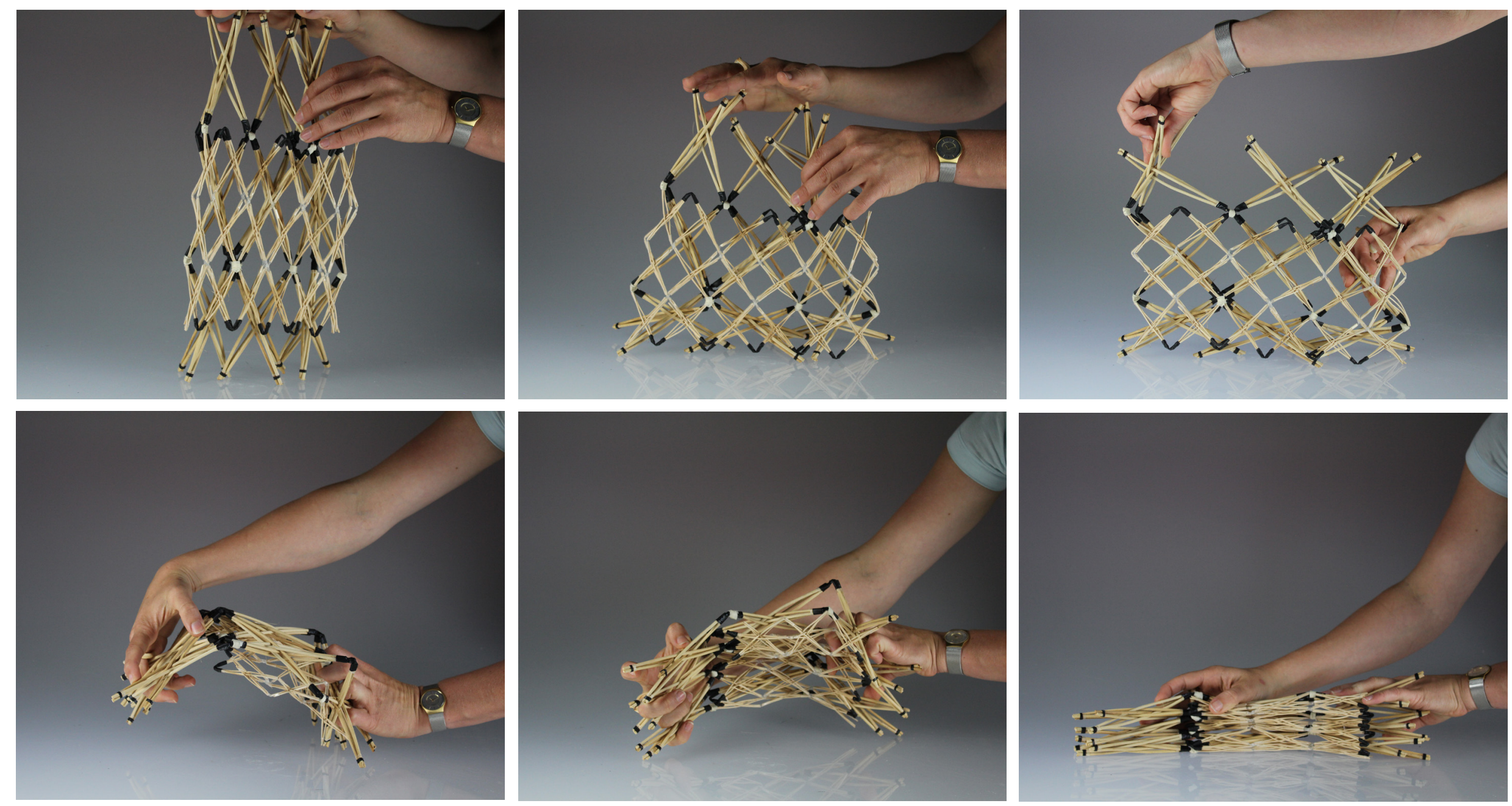

Figure 5.18

Continuing from the storage weave development I began to explore how multiple scales of weave could be connected to create a smaller more tactile surface for that shelters that require more storage or comfort. 


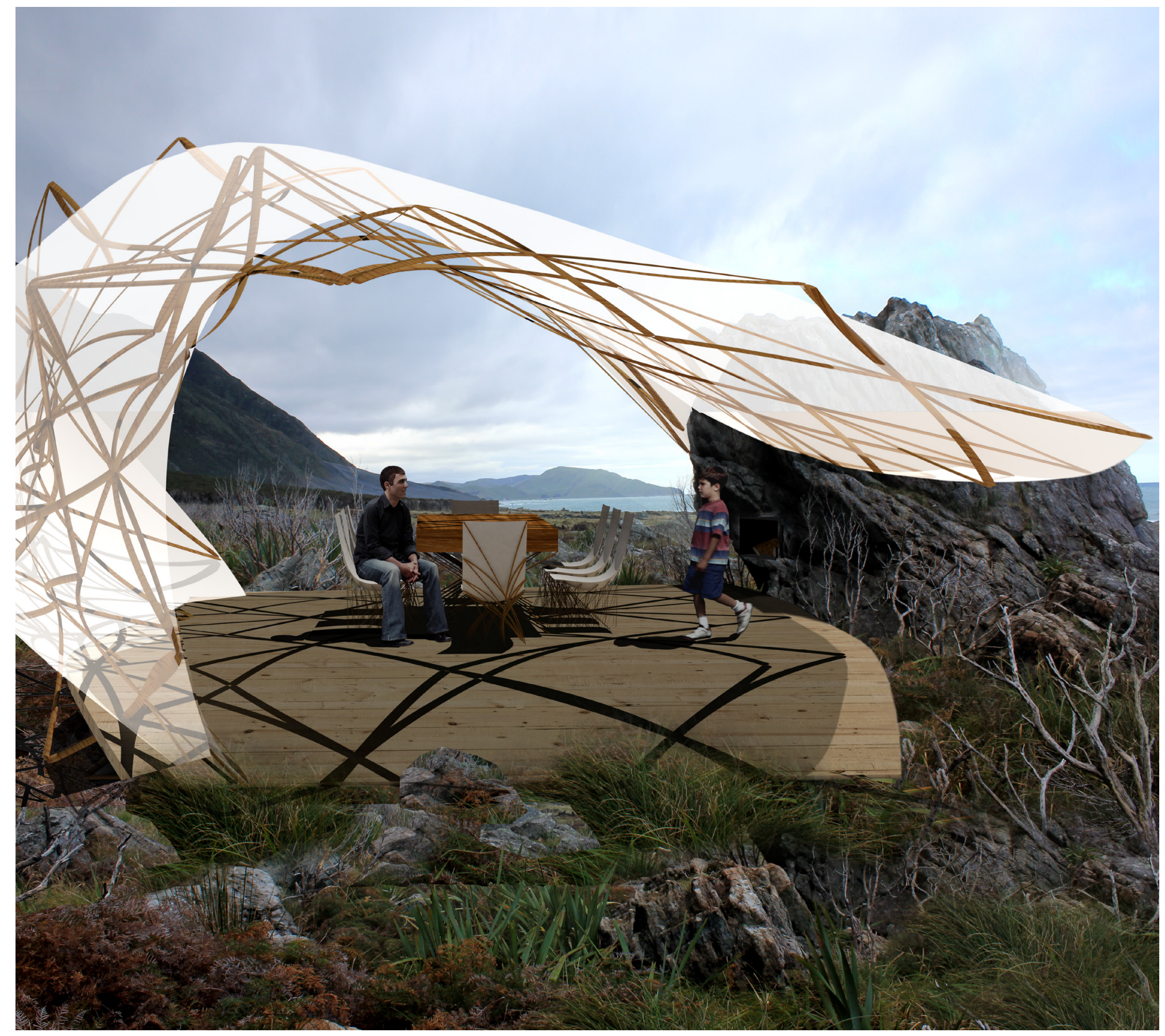

Figure 5.19

This perspective shows a stage in the developed design. The decking floor system aligns with the weave and floor detail development on the following page.
The furniture, number of structural systems and connection to the environment is altered in the final shelter design to further emphasise the connection to the surrounding context. 


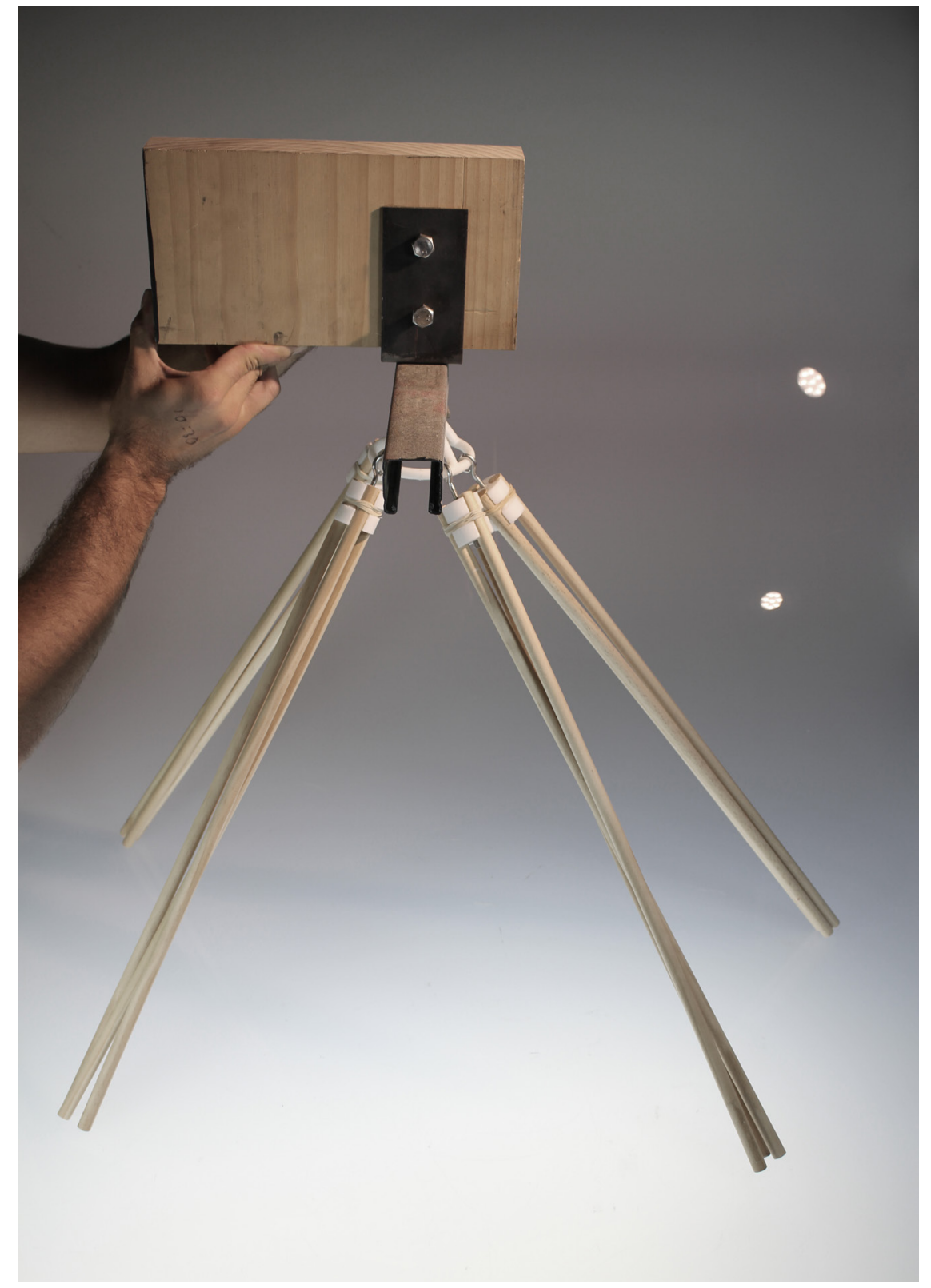

Figure 5.20

This physical model was constructed in an attempt to resolve the connection between the stick weave members and the floor joists for the decking.

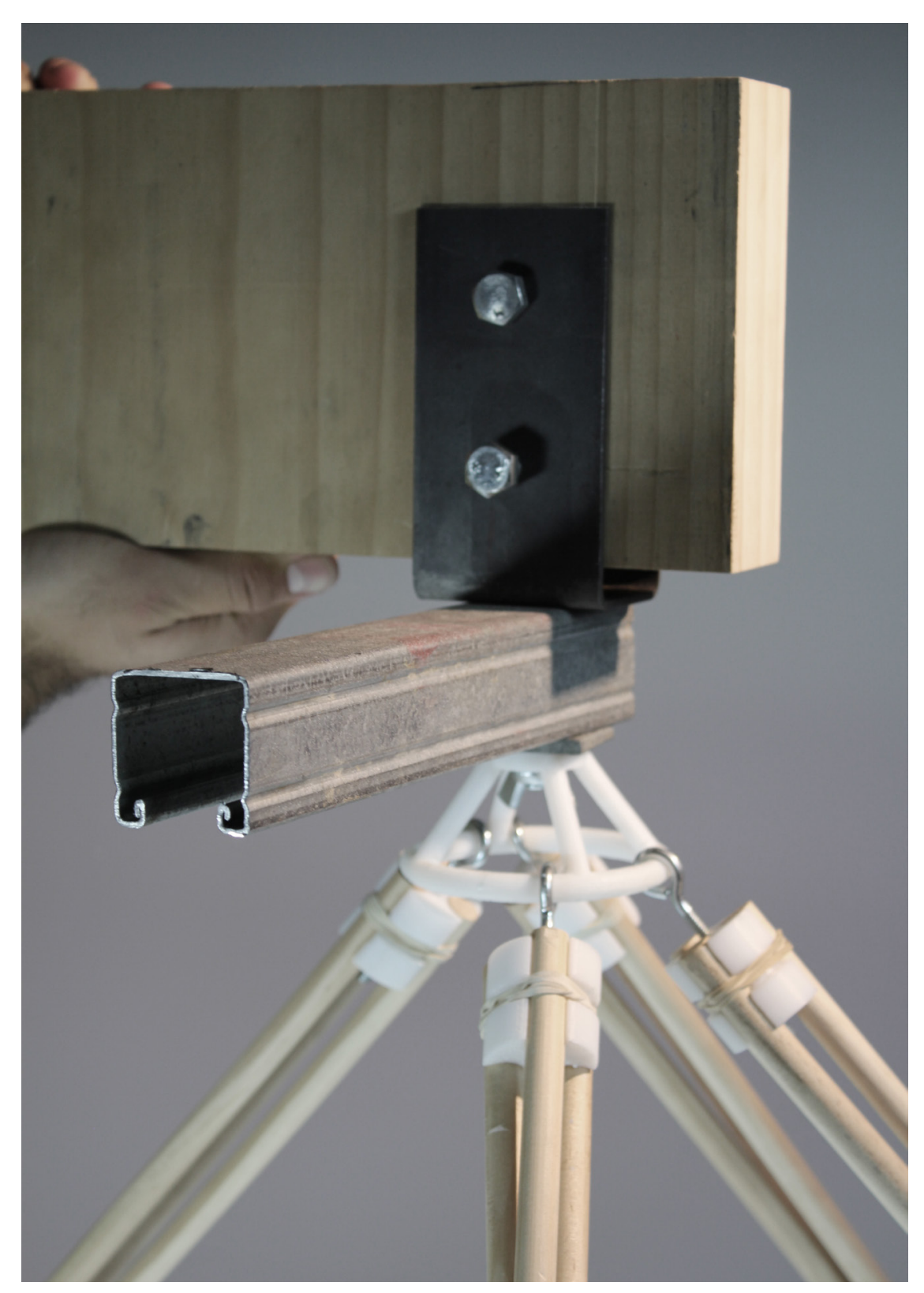

Figure 5.21

Close up view showing the steel section and components that were modelled in Solidworks and 3D printed with white nylon plastic. Another method of rapid prototyping in the digital realm. 


\section{Final Shelter Design}

This concept of removing designer control opposes the very nature of architectural design as a balance between function, experience and mitigating risk through controls such as building codes. The development of a narrative detailing how a person would enter the site, navigate the landscape and occupy the building as a part of their daily activities helps to define all functional design needs of the shelter. A reversion to the split shelter arrangement is explored in this section through writing, hand drawing and finally connected with the digital modelling process for form the developed design.

A detailed narrative followed the movements and outlined the needs of the users of the shelter, refer to Figure 5.22). This was expressed through a succession of diagrammatic studies relating to the boulder site that again focussed on maximising programme for its non-static potential. The divergent plan explored in Chapter Two is revisited here where each function is separated into isolated shelters. Again the shelters are positioned with large enough expanses in between to force immersion in the environment while allowing a sense of ownership over the transitional space. Each shelter is positioned to visually force connection with architecture and wilderness. This is achieved through careful arrangement of each shelter so that all view shafts look from interior to frame another shelter as well as the coastline. Revisiting the framing analysis from the initial design tests, specific view shafts are designed to maximise the feeling of being within the landscape instead of disconnected from it as well as reinforcing the wilderness design dialogue.

The final design is described visually through the plans, sections, details and perspectives to follow, (Figure 5.23-5.27)

Figure 5.22 (right)

Narrative mapping of boulder site.

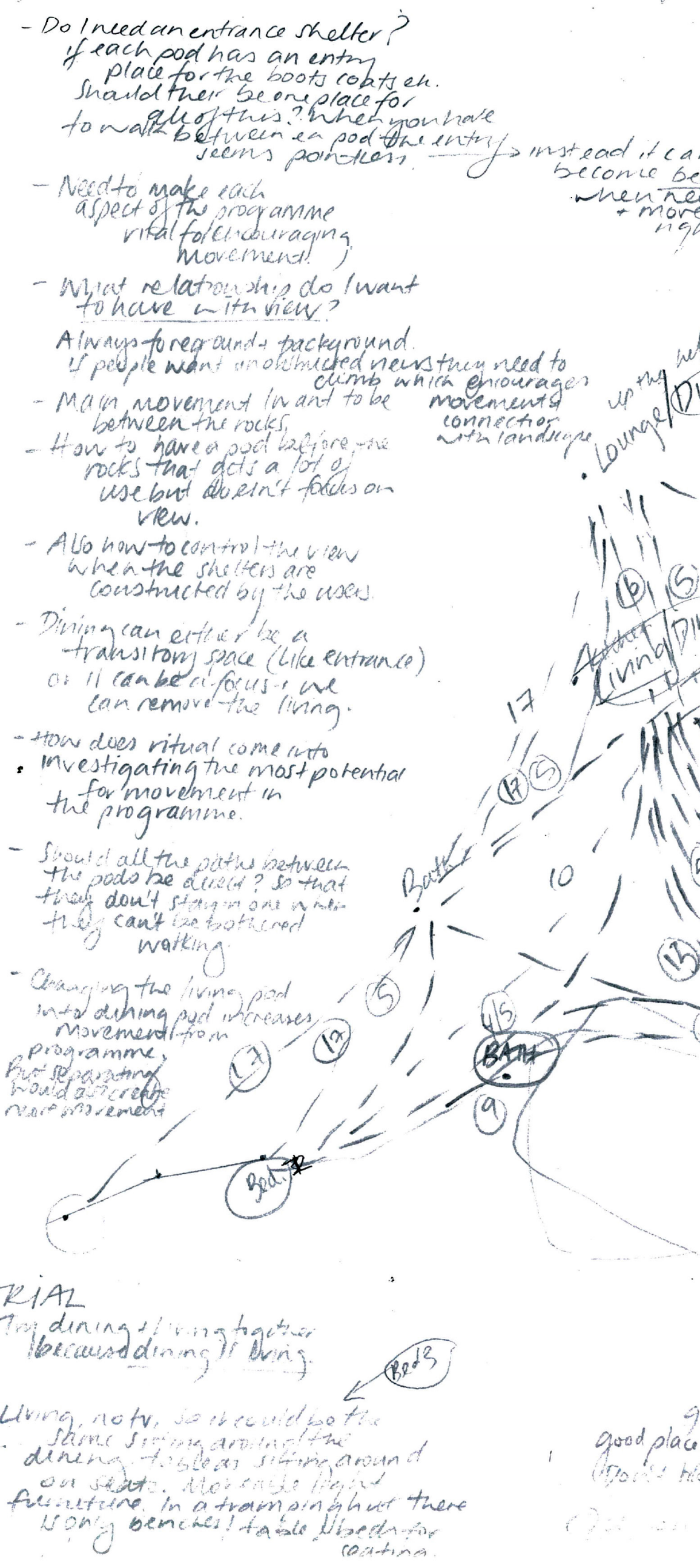

- Do Ineedan entrance kelter?

place for has an entry

hasld their se one place for

to wallojthis? Ihen noutrale

Jecreem en pod the ente

wento make tan

vital foicluaraging
movement?

list relatious stes do lwant

to have sita bien?

Aivays foregound tacteyriund

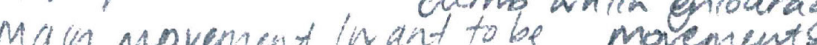

connectior

on to have ope betine w

ocks that dets a loy

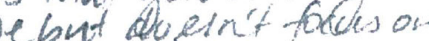
rtew.

Also how to contry tias bu hen the shither are

enturated by was

ti can be ri grus he

can remove tive living

Has dues nitual come unt

Mvestigatingtire most porentiar

the for movemeat in

programme

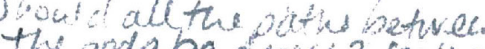

a cant be ?atin

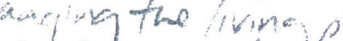

movementlof

prosiamme

rovisuting connectio

ces 

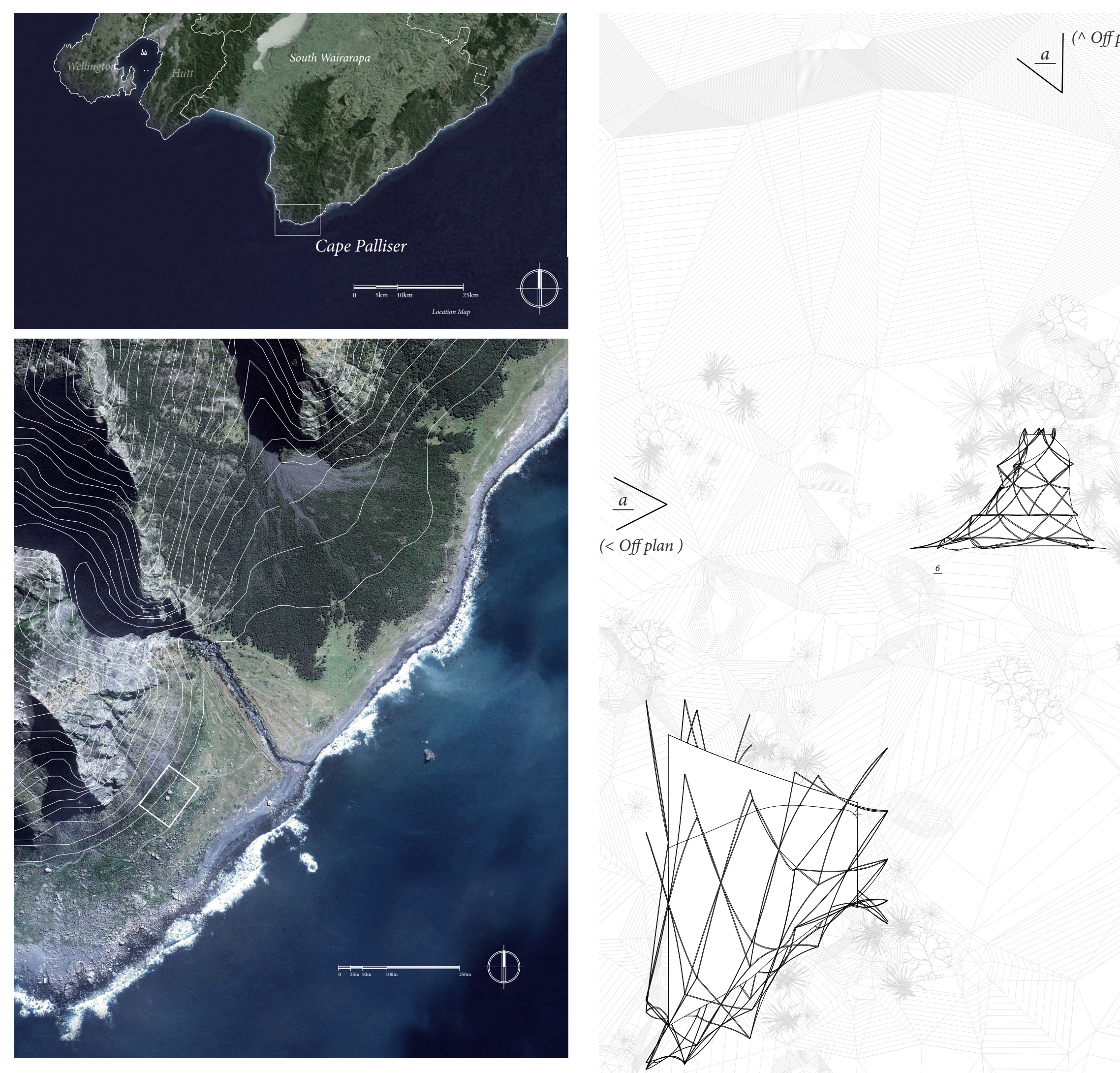

$$
\frac{a}{\left.\right|^{(\wedge O f f p l a n)}}
$$

Figure 5.23

Top - Location Plan, Bottom - Ngapotiki Reserve Site Plan 1:10000

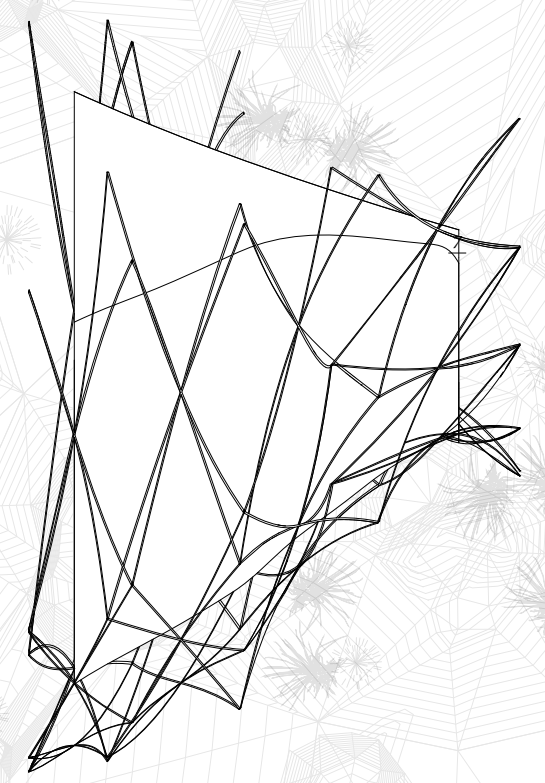

Figure 5.24 (right) 

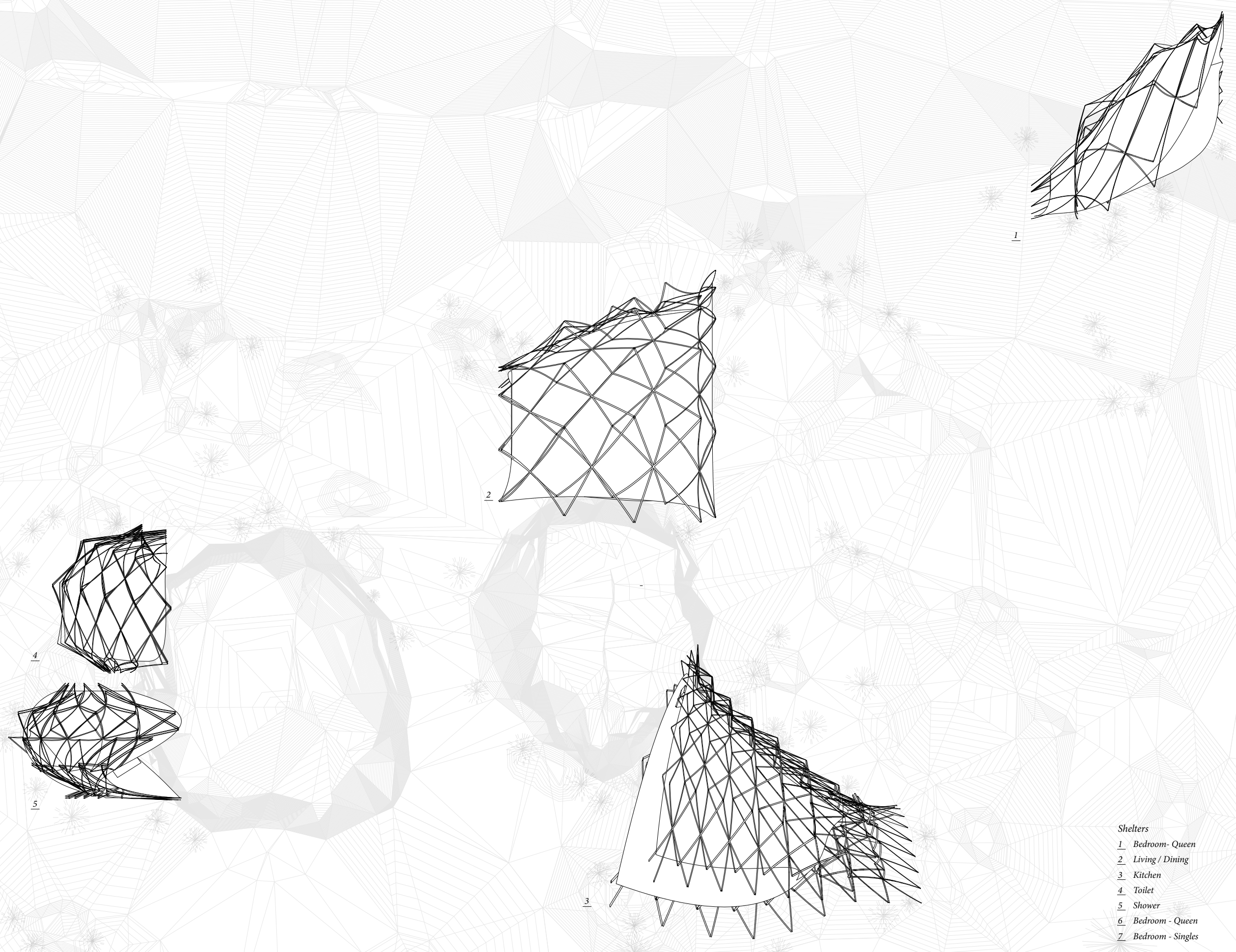

Shelters

1 Bedroom-Queen

$\frac{2}{2}$ Living/Dining

3 Kitche

4 Toilet

$\underline{6}$ Bedroom-Queen

$\underline{7}$ Bedroom-Singles

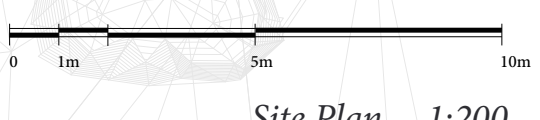

$\varnothing$ 


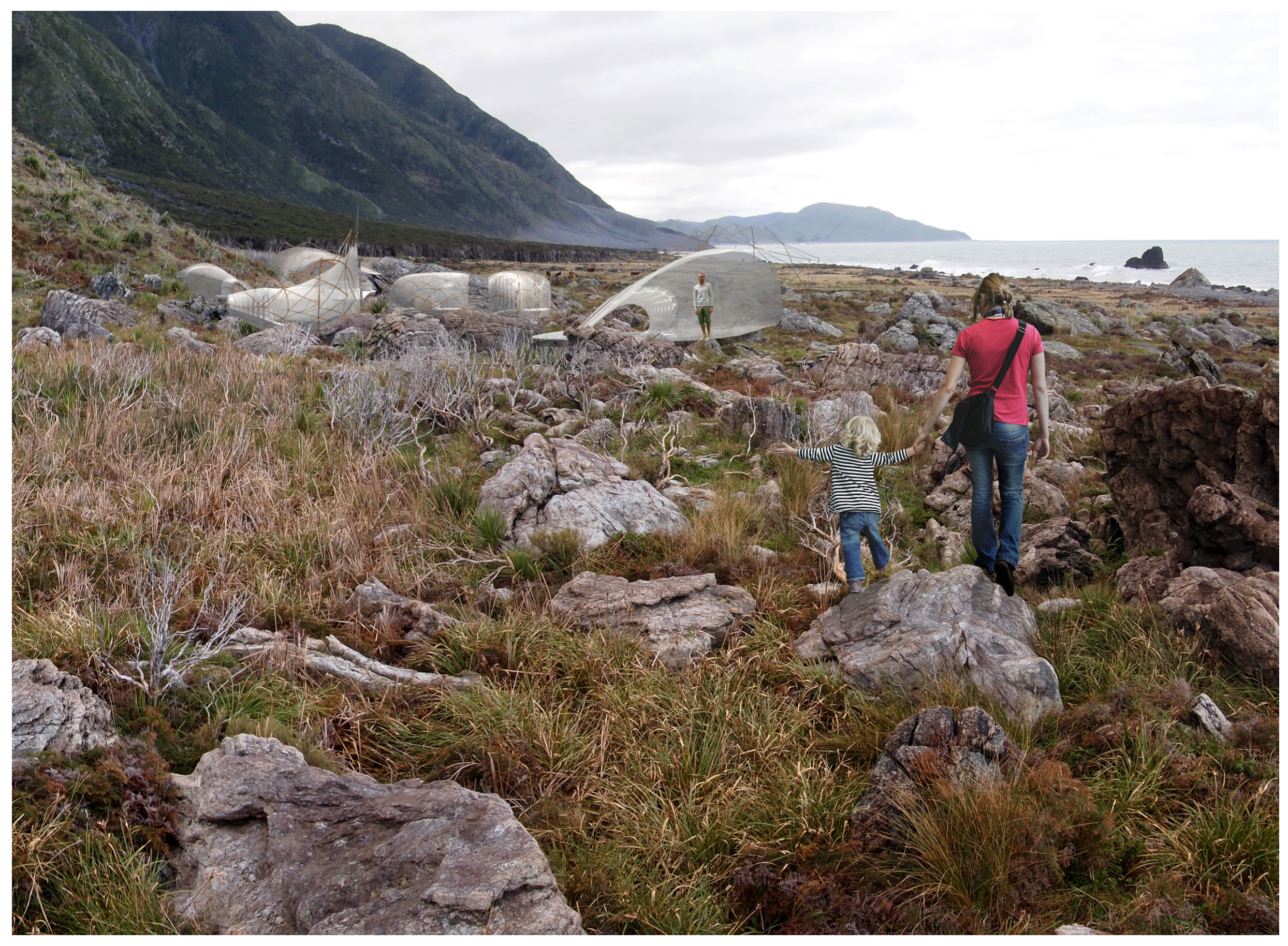

Figure 5.25 Perspective a, (position indicated on Site Plan)

The shelters are displayed within the context of the wilderness site in this long distance perspective. 


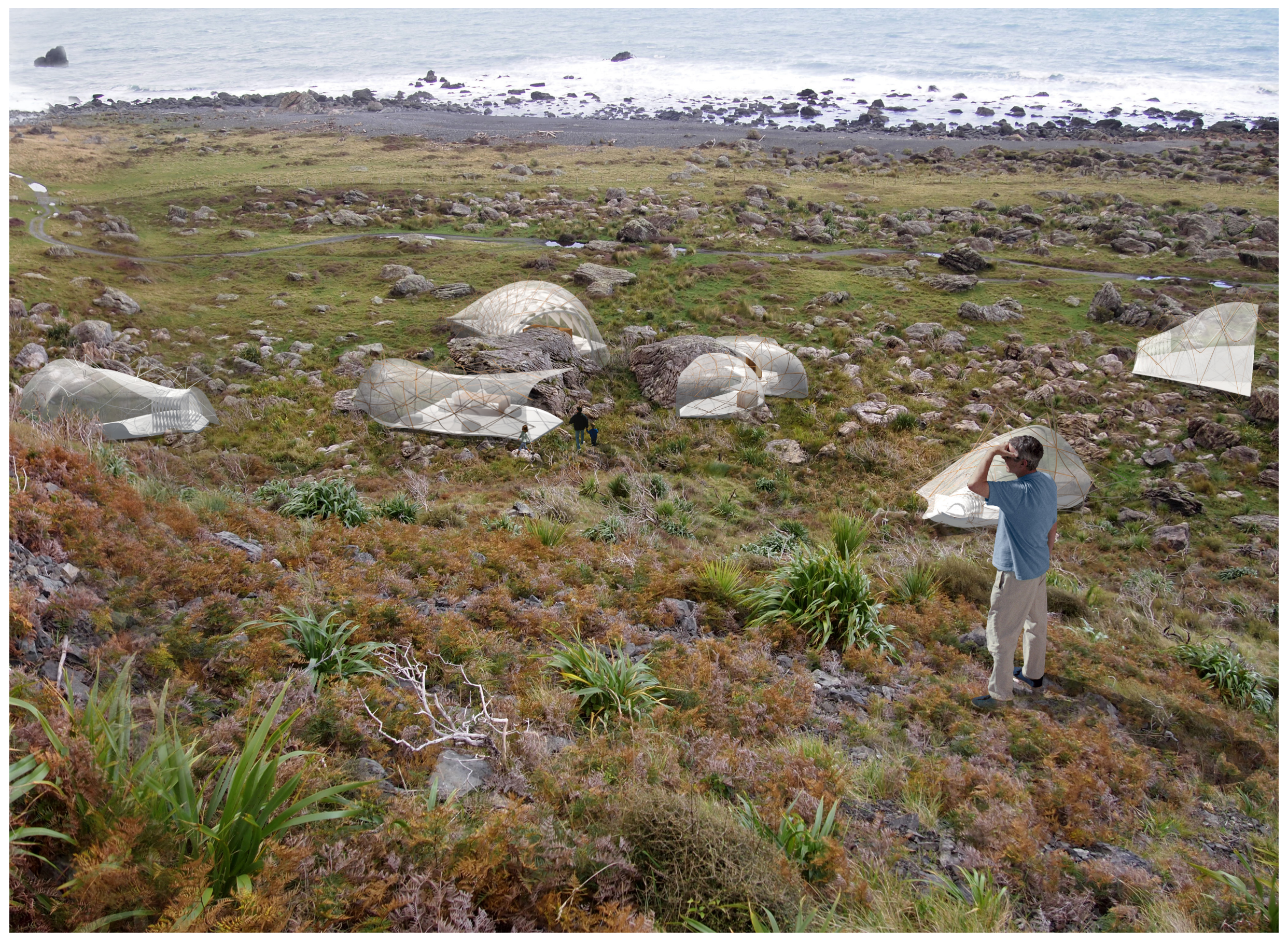

Figure 5.26 Perspective b, (indicated on Site Plan) This perspective displays the view from the steep hillside behind the shelters, down to the flat rocky coastal platform and out to the coastline. 


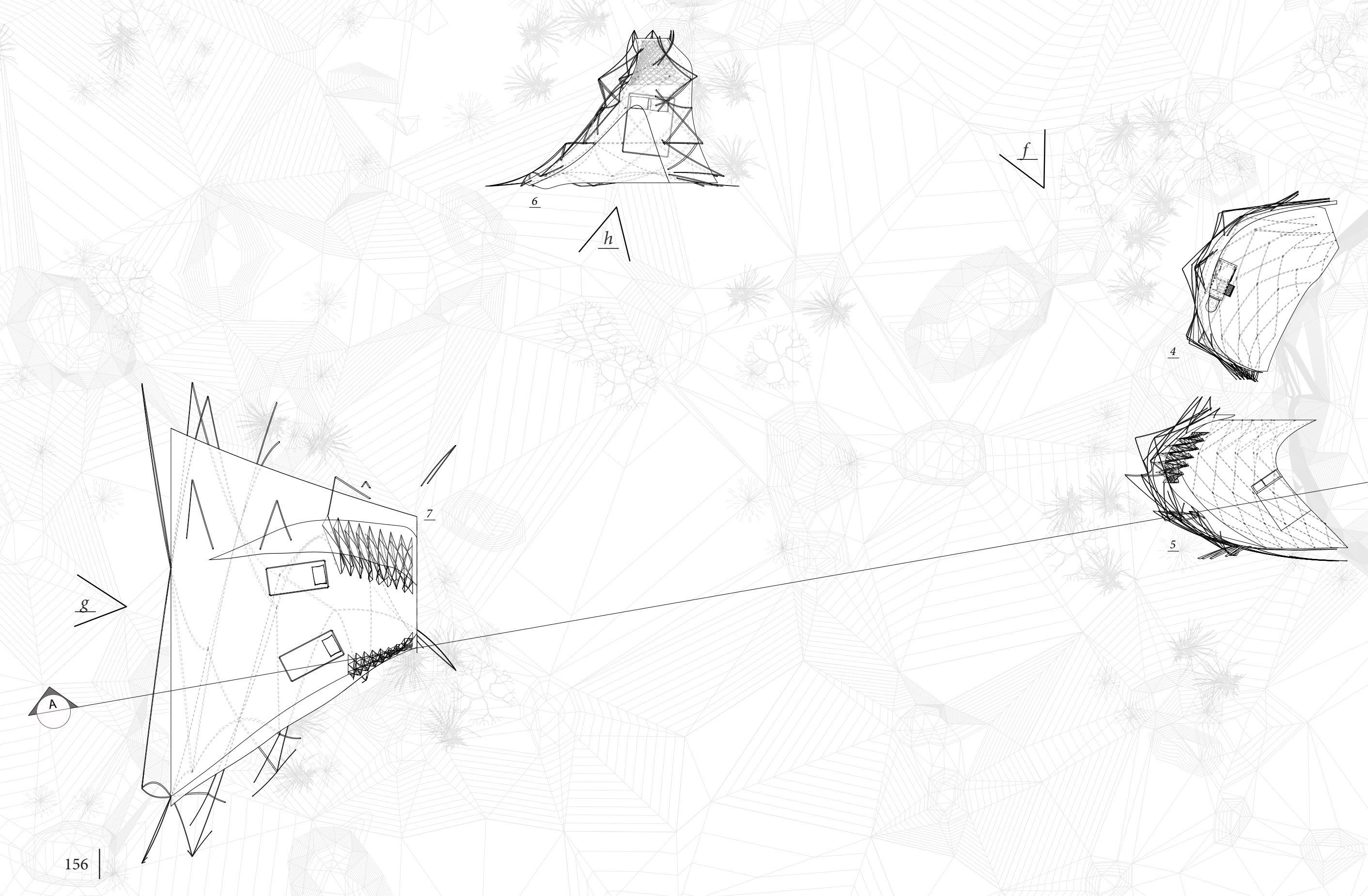


$\angle$ 
The function of each shelter becomes obvious through the prefabricated utilities pods that are placed within. These transportable, plumbed and wired pods are designed to be trucked or flown to site and act as permanent elements within the architecture, with the exception of the living/dining space where the utilities are semi-permanent, refer to Figure 5.28-5.31.

At this Nga Potiki Reserve site the most obvious quality of the landscape is movement and change. There is a constant interplay between permanent, semi-permanent and fluid features. The architecture is designed to follow this scale, with permanent insertions into the large rocks; semi-permanent flooring and utility pods sitting amongst the uneven vegetated ground plane, and fluid movement of the stick weave being transformed by the wind and people as they interact with the architecture.

The shelter aims to leave only designed alterations to the landscape. The programme is designed to emphasise sustainable use of natural resources such as water and power, and employs a range of waste treatment systems that minimise impact on the environment.

The utilities pods emphasise the relationship between function and experience by challenging preconceived notions of architecture through juxtaposition with the fluid changing stick weave. The weave is free to move uncontrollably as it interacts with the conditions sometimes rendering the utilities unusable. The building is then altered by the occupants in response to these environmental changes highlighting the ever present and often invisible suppressed relationship between culture and nature.
Topography Ground Plane

Large Rocks

Smaller Rocks

Ground cover

Trees

Plants

Flaxs

Tussocks

Water

Wind

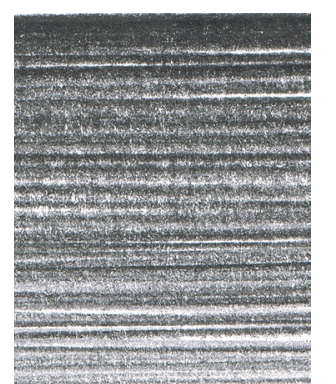

Utilities Insertions

- Kitchen Stove

- Living Fireplace

- Sink Bathroom

Utilities Pods

Floor Platform +

Stickweave structure to support it

Table

\section{Stickweaving}

Architecture

\section{Dining Chairs}

\section{Occupants possessions \\ - Boots \\ - Packs \\ - Clothes}

Stickweaving Membrane People 

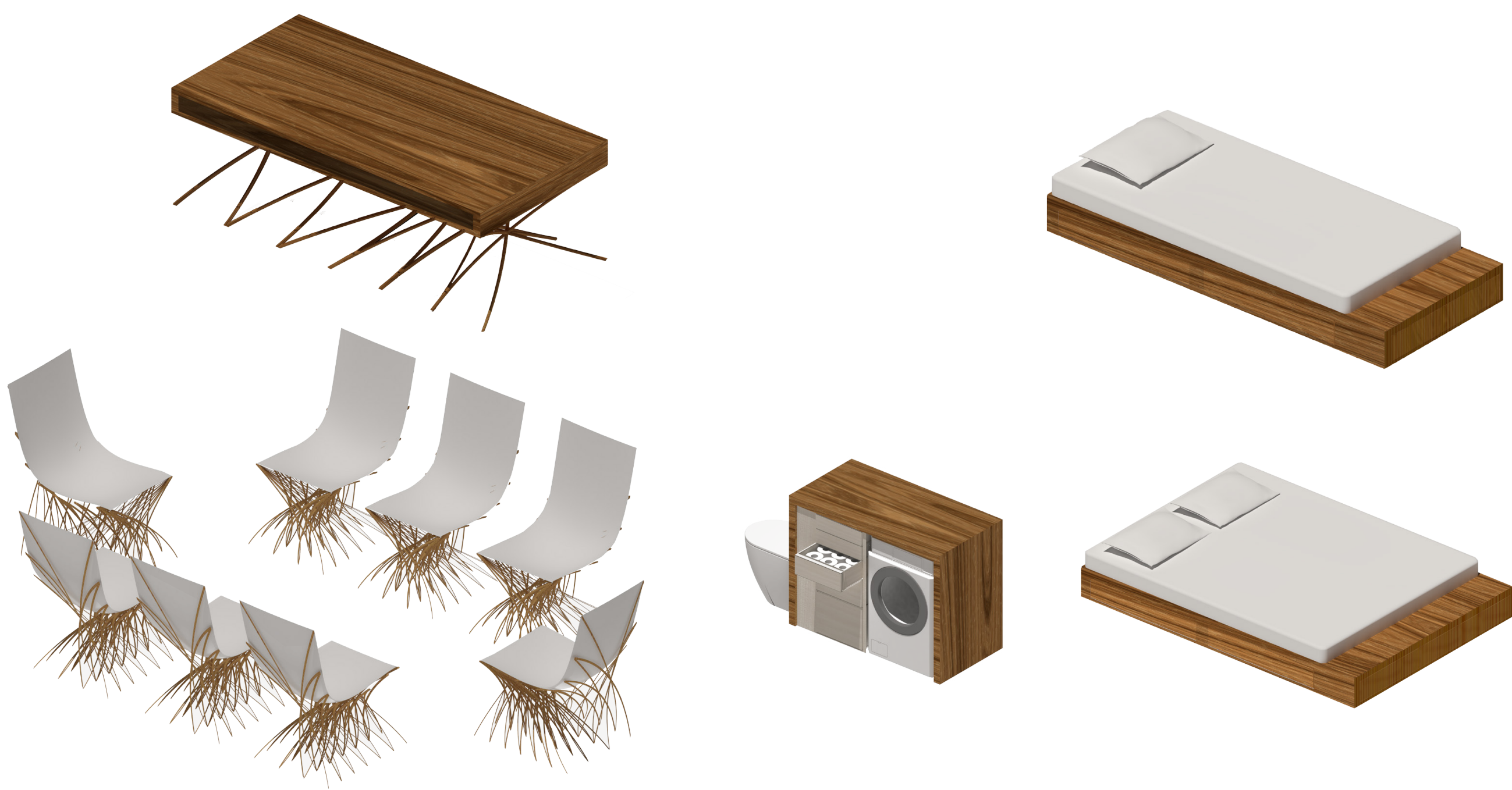

Dining Table

Adjustable height

Storage

Toilet/laundry

Toilet

Storage

Washing Machine

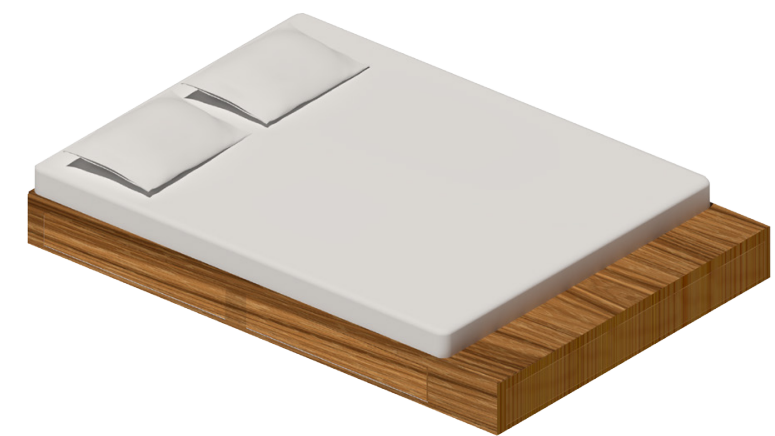

Chairs

Seating

Rock sink - permanence

Figure 5.28

Utilities Pods - Iteration One

These units contain all necessary elements to make each space function within the programme. 


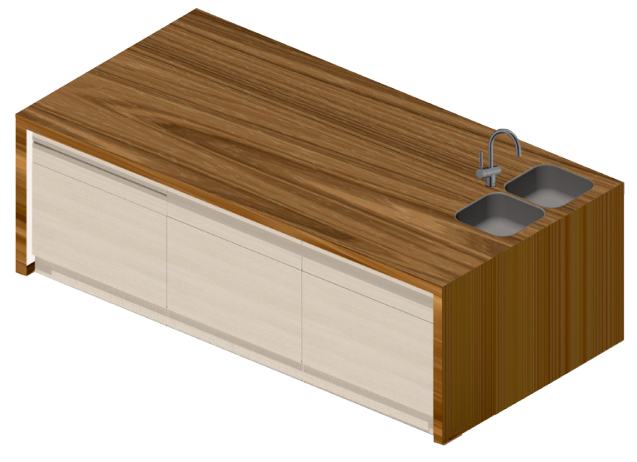

Kitchen

Fridge/Freezer/Pantry Drawers

Sink

Oven

Kitchen Storage

Hot plate barbecue - permanence

Figure 5.29

Utilities Pods

Each utility pod is designed to speak of glamour through refined detailing. High quality products, materials, and finishes reinforce this.

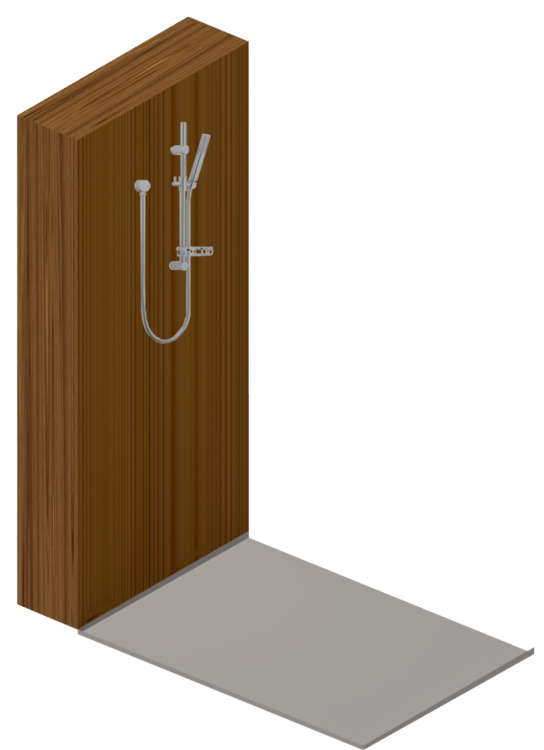

Shower

Shower

Rack

Storage

Tilt floor rock drain - permanence
Timber joinery in Hinau (lighter) and Totara (dark) relates to the surrounding environment as these timbers can be seen in the forests across the site. 


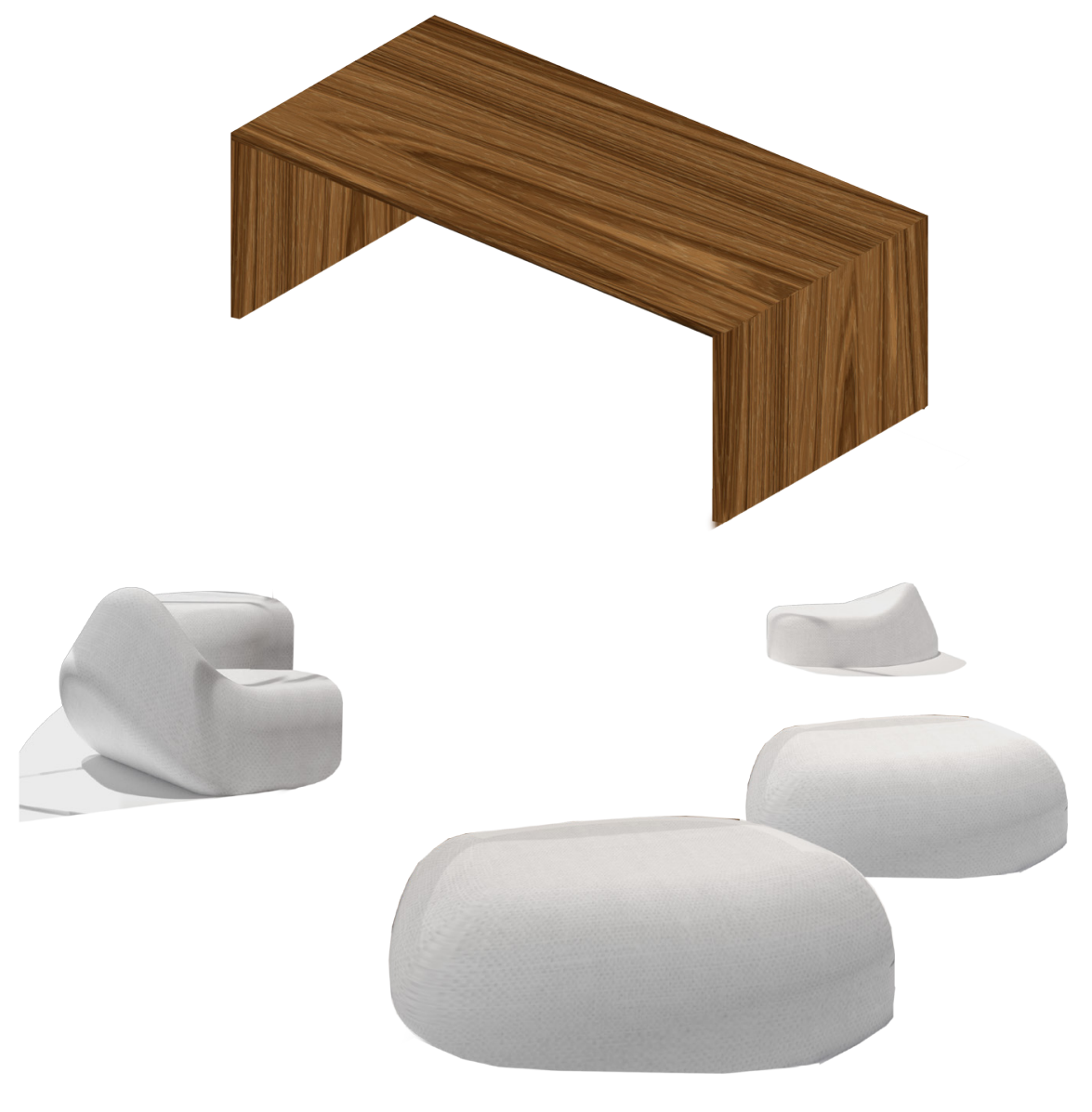
Dining Table
Adjustable height
Storage

\section{Chairs}

Seating

\section{Figure 5.30}

Utilities Pods - Iteration Two

These revised utilities simply the spaces and are designed to encourage inhabitation of the ground plane. The chairs are formed from memory foam, a similar material to the compressed foam floor surfaces.
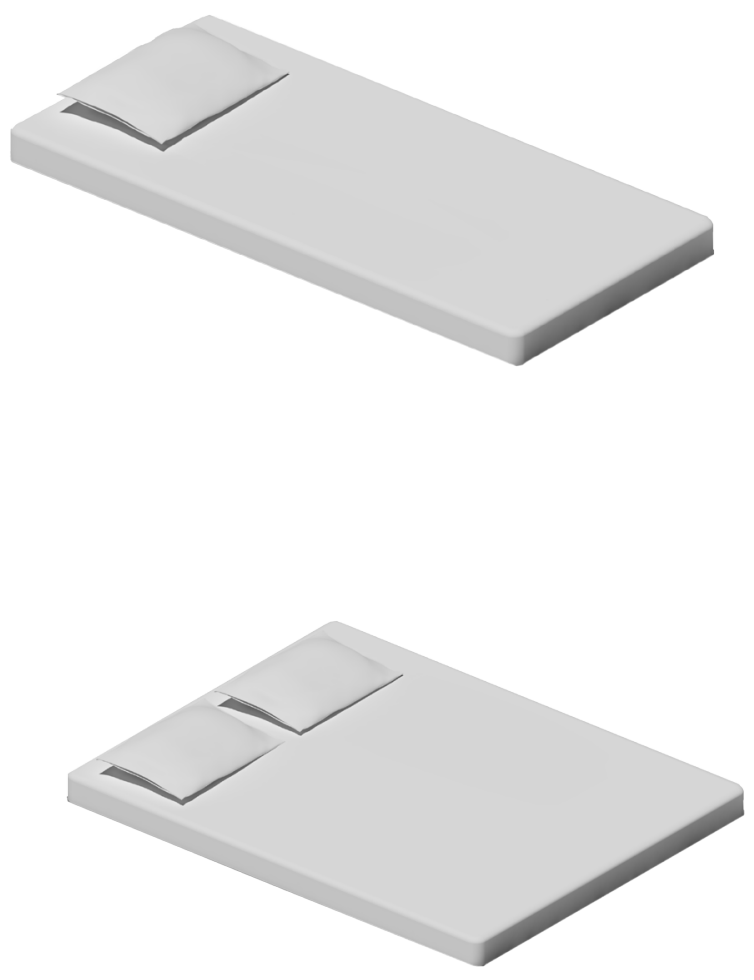

\author{
Queen + Single Beds \\ Clothes Storage \\ Bedding \\ Mattress
}

The timber bases of the beds were removed as this also reinforces inhabitation of the floor plane by bringing the users closer to the surface. 
Stick weaving structure base net compressed

Utility pod

(kitchen) packed

Floor surface - stacked

Weave surface+ ground plates packed for transportation

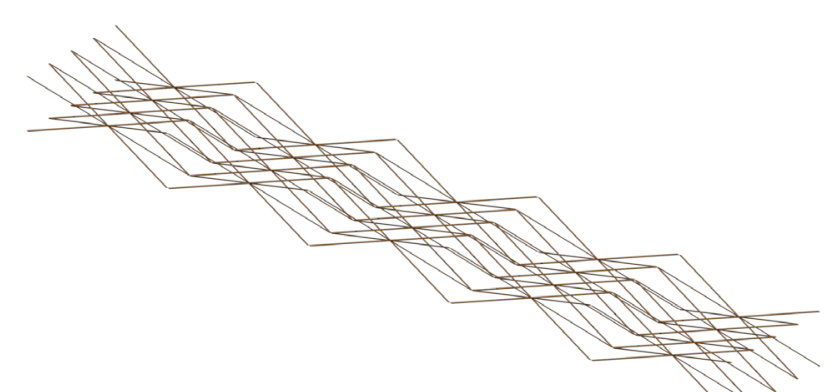

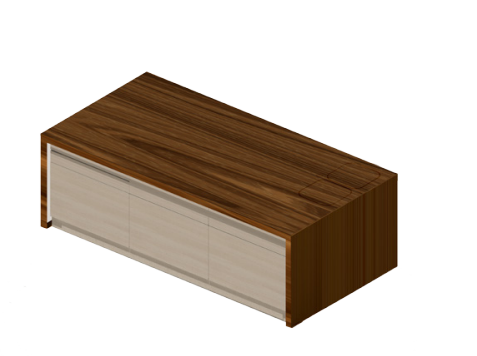
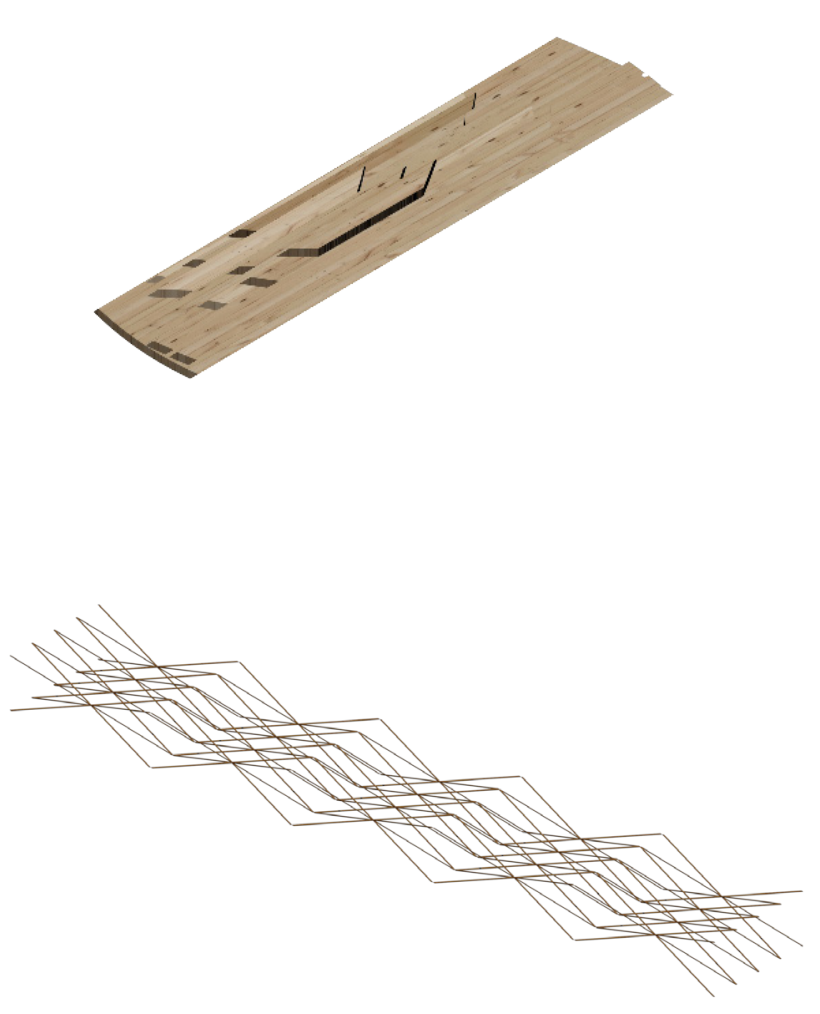

Stick weaving structure base net uncompressed

\section{Utility pod \\ (kitchen) open}

Floor surface - half constructed

\section{Figure 5.31}

Initial developed construction sequence exploded axonometric of Kitchen components. Each of the systems is designed to be semi-permanent and transportable to enable placement at any end-of-road site. The construction sequence and parts are shown.
This sequence was later revised as each shelter was simplified into one surface of weave and floor instead of separate weave structures for floor and canopy. The construction sequence still remains predominantly the same. The floor surface is instead inserted into a pocket in the weave membrane and then connected to the weave at the edges.
Weave surface and ground plates half constructed 


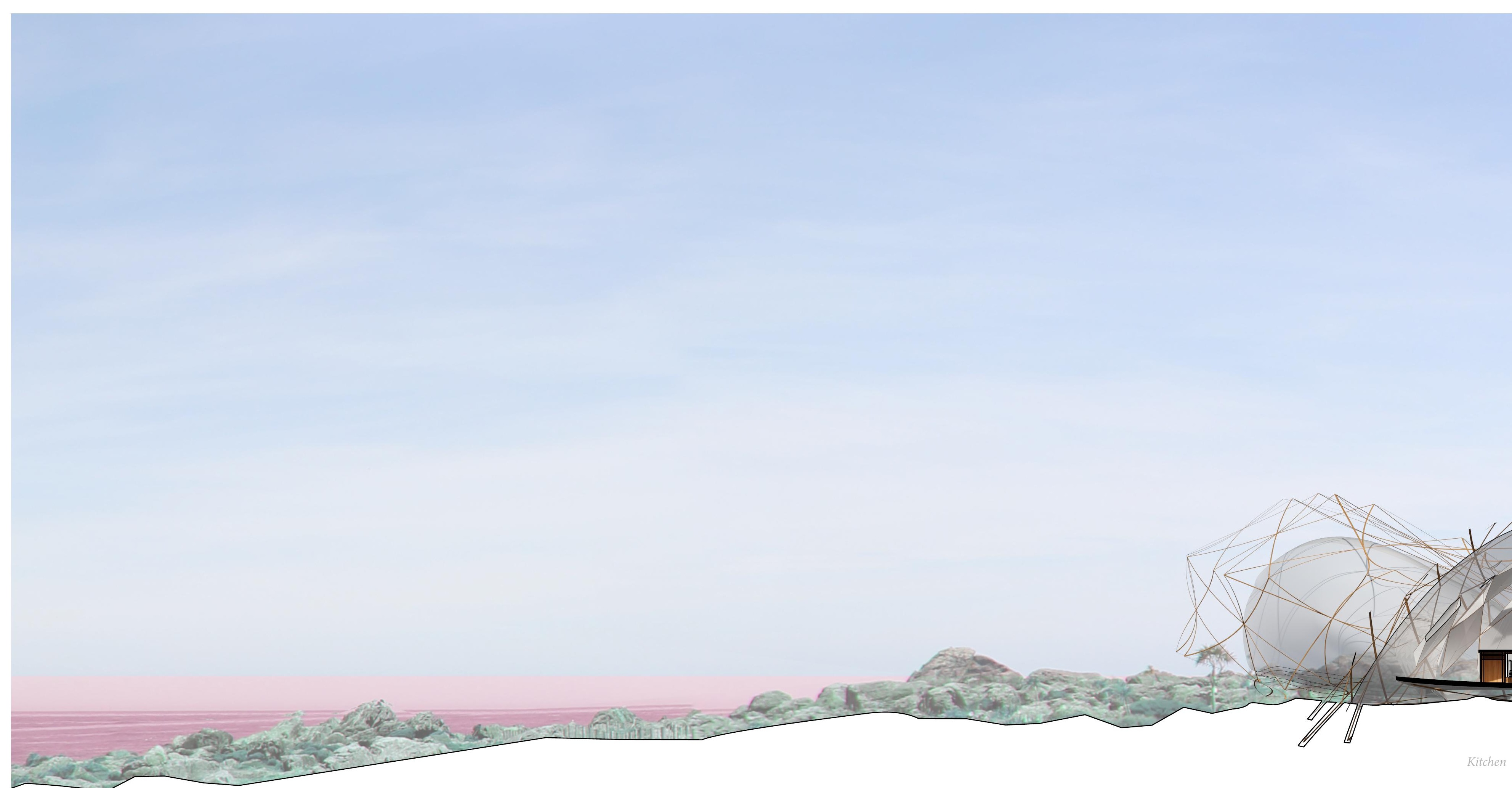

\section{Figure 5.32}

Cross-section 1:100

Section through kitchen and living space displaying the permanent

insertions into the boulders and the relationship between semi-permanent 


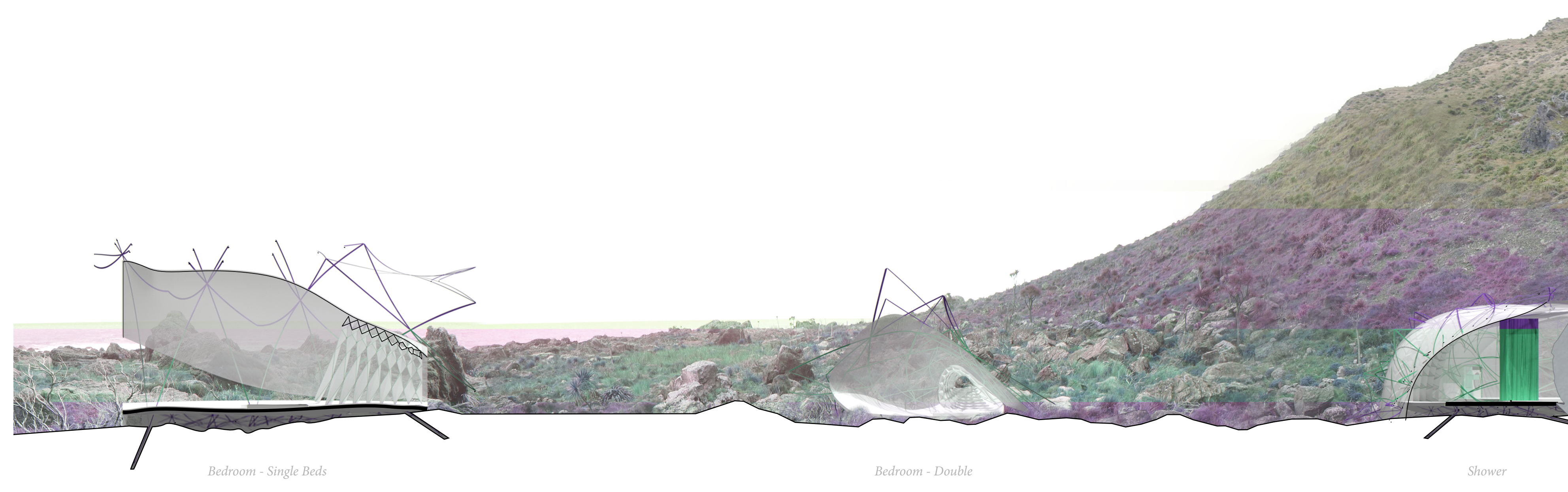

Figure 5.33

Longitudinal Section 1:100

Section displays the relationship between shelters and distances between

that encourage movement and ownership of the wilderness. 


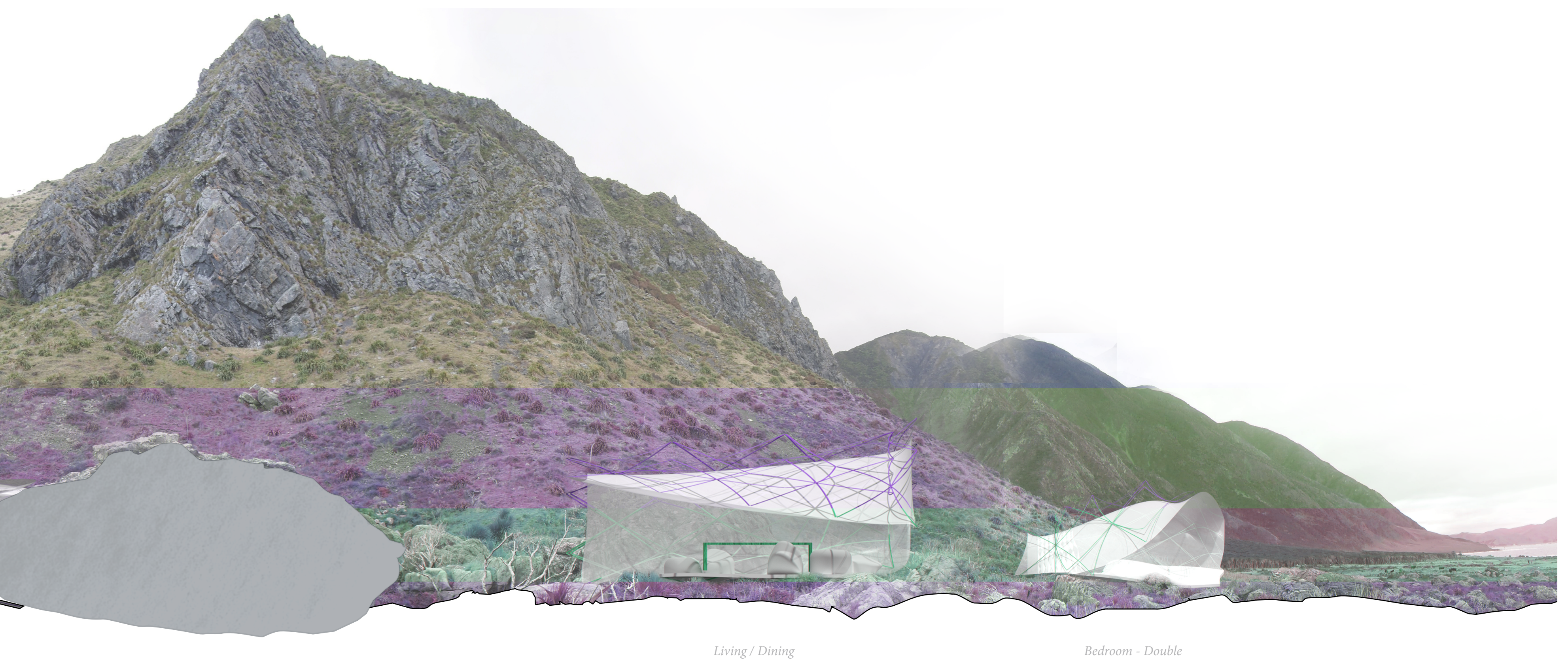





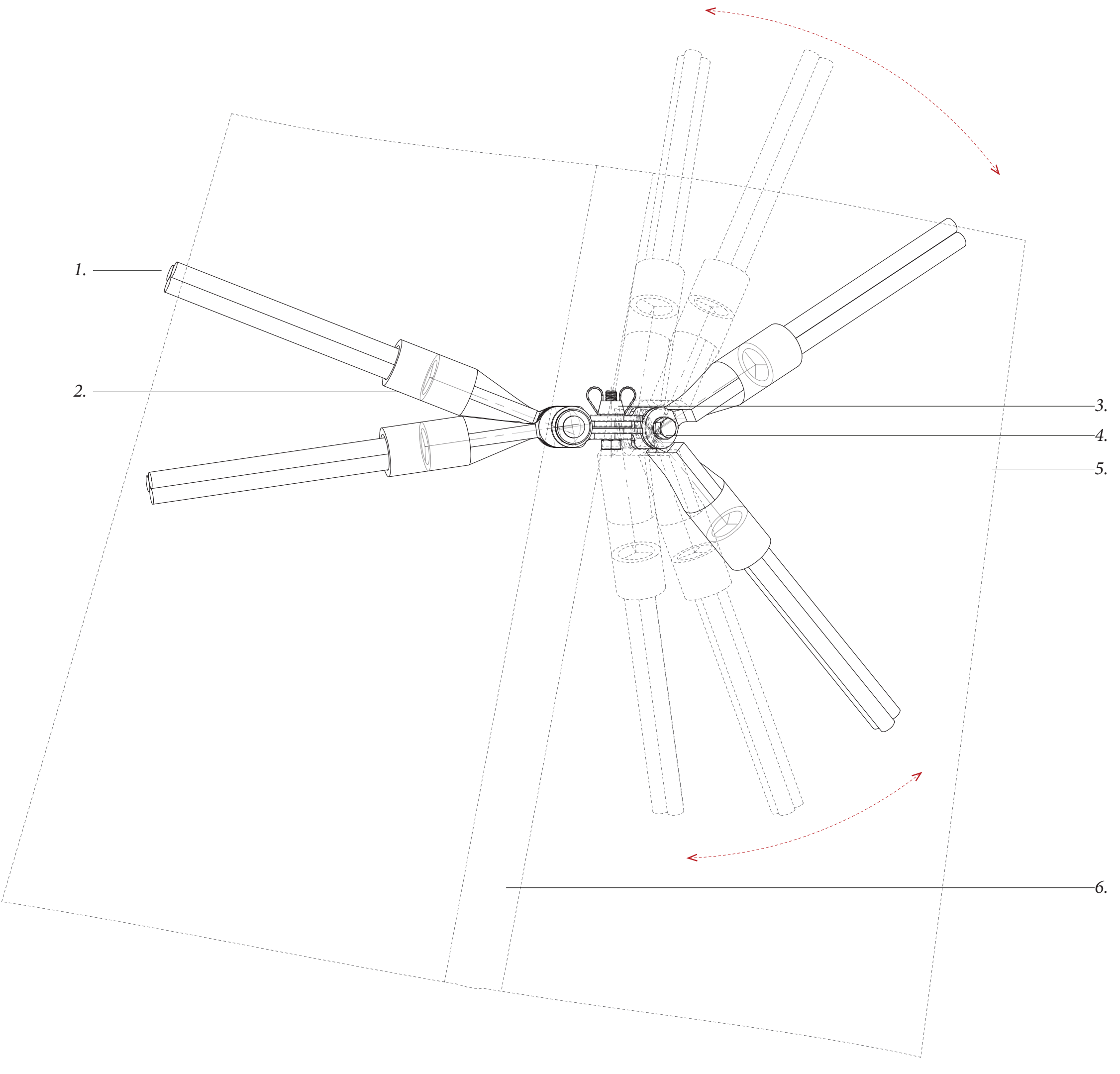




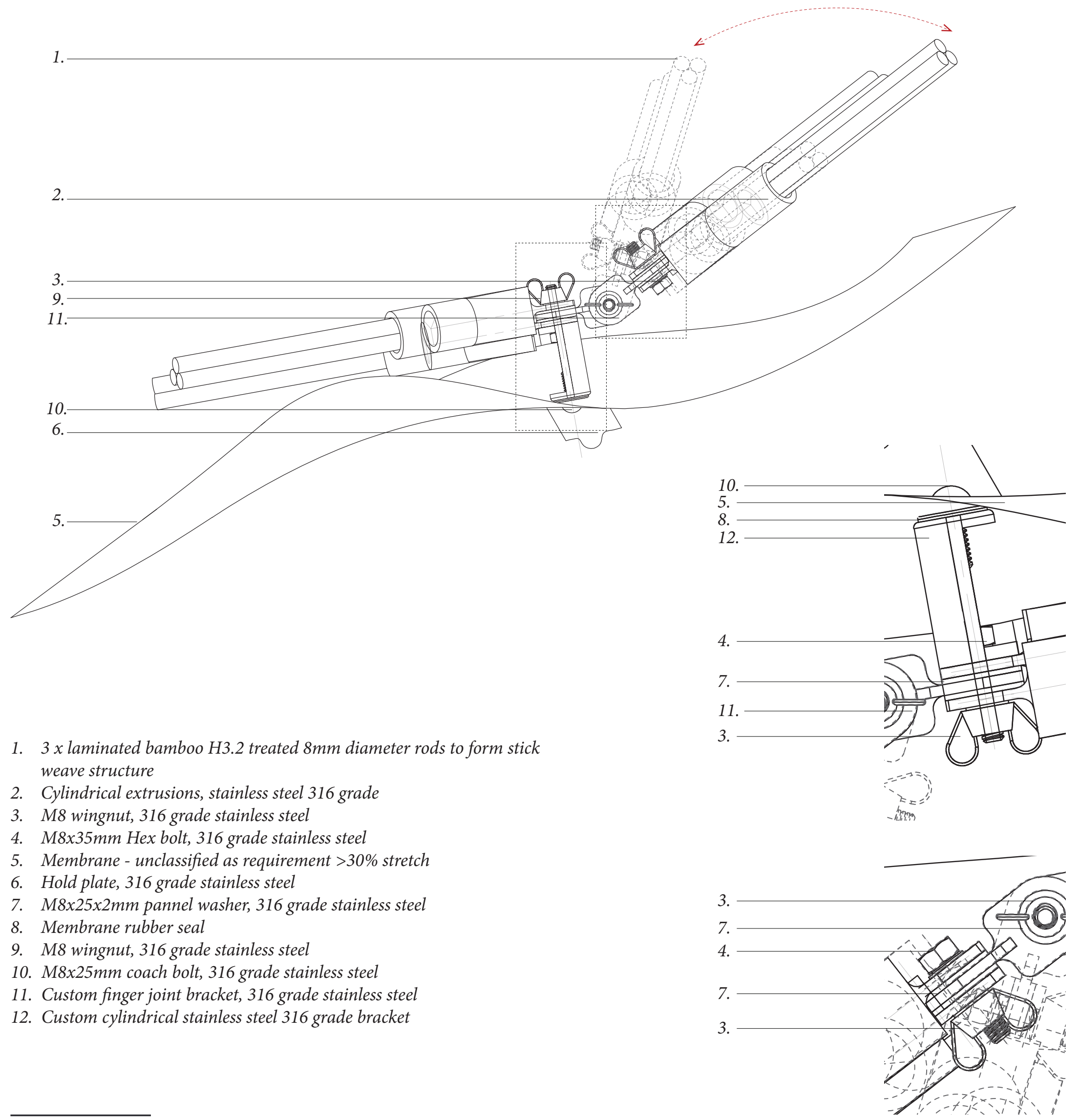

Figure 5.36

Detail One - Elevation 1:2 


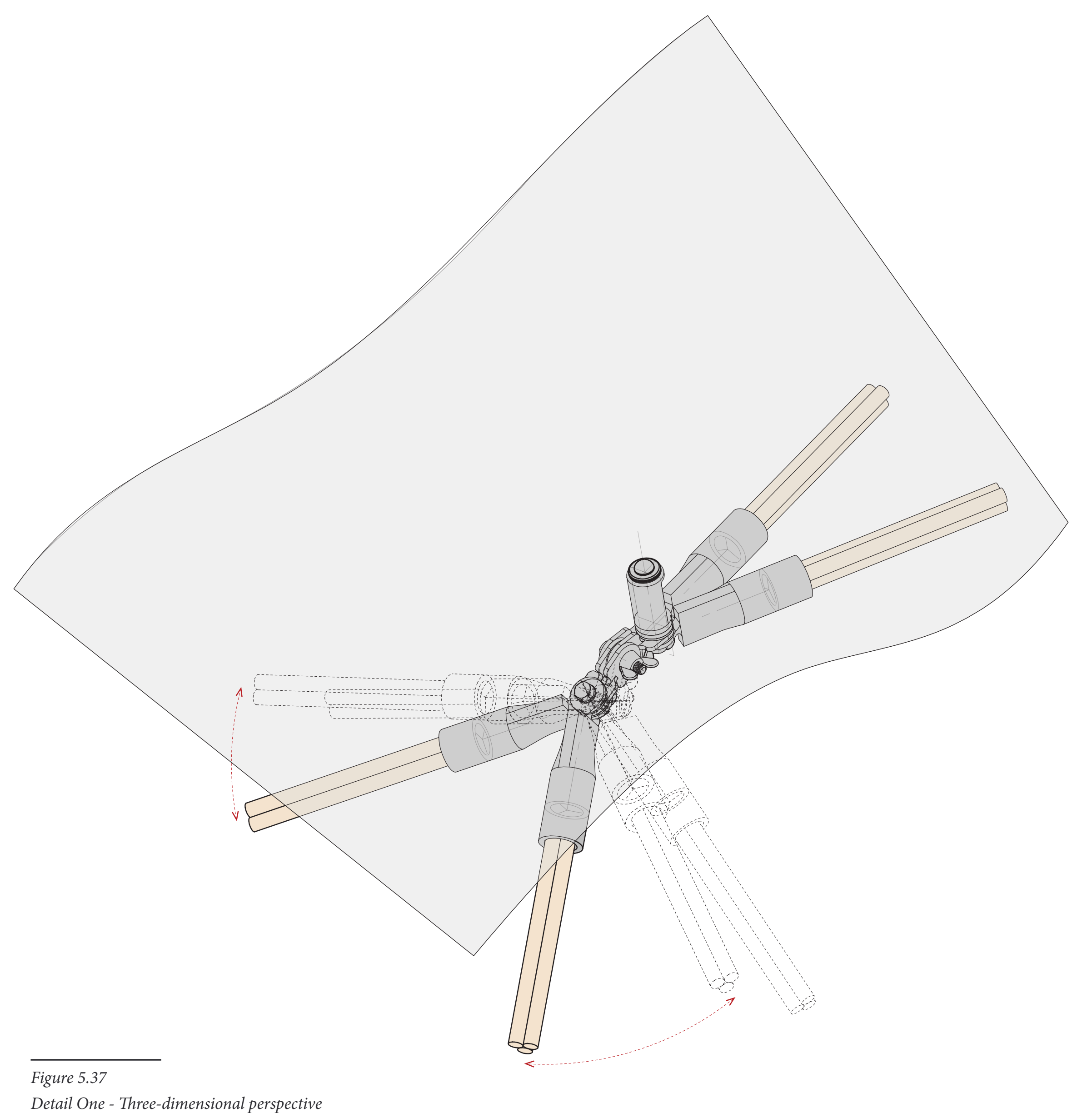




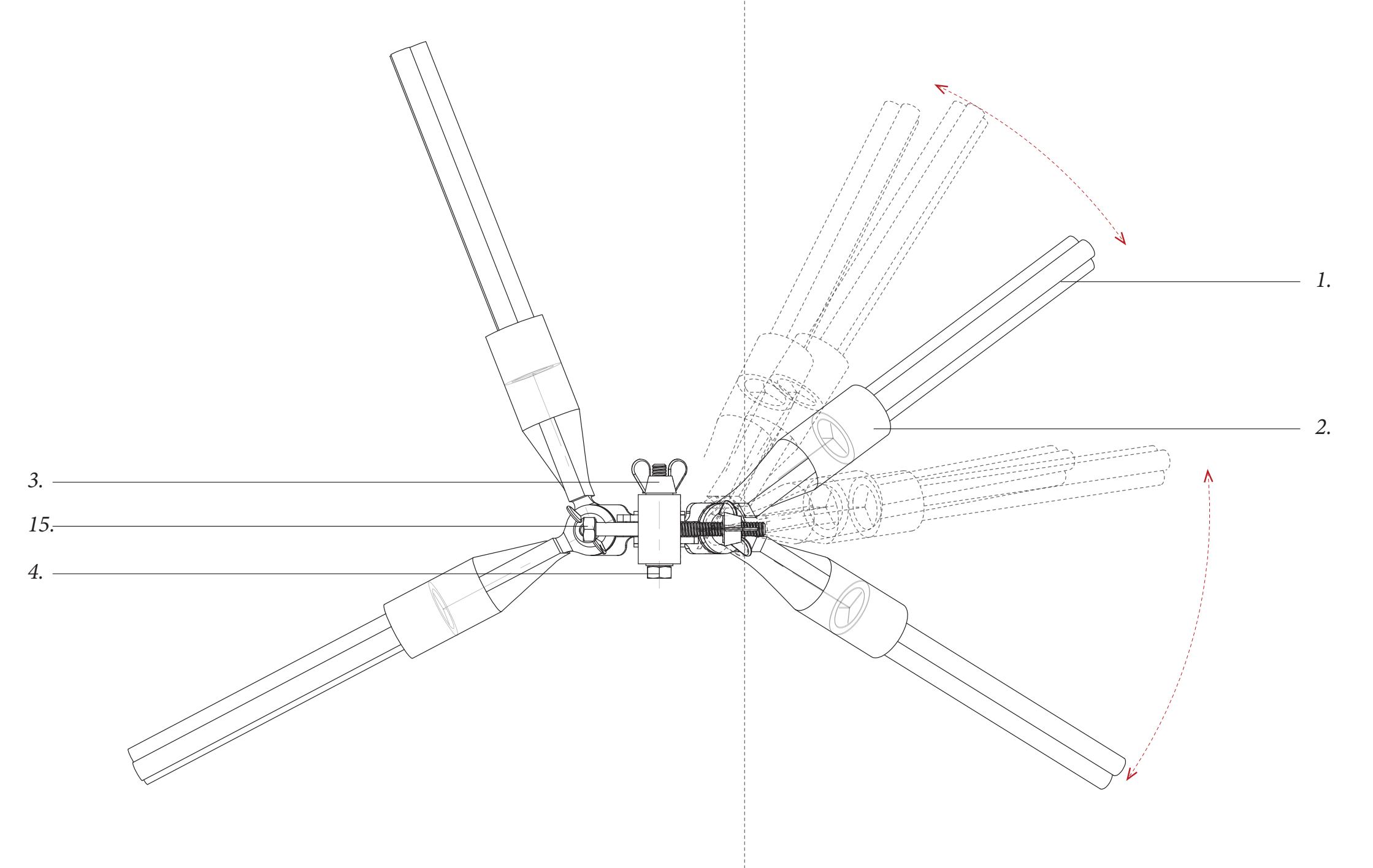


1. $3 x$ laminated bamboo $\mathrm{H} 5$ treated $8 \mathrm{~mm}$ diameter rods to form stick weave structure

2. Cylindrical extrusions, stainless steel 316 grade

3. M8 wingnut, 316 grade stainless steel

4. M8x35mm Hex bolt, 316 grade stainless steel

5. Membrane - unclassified as requirement $>30 \%$ stretch

6. Hold plate, 316 grade stainless steel

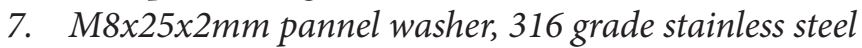

8. Membrane rubber seal

9. M8 wingnut, 316 grade stainless steel

10. M8x25mm coach bolt, 316 grade stainless steel

11. Custom finger joint bracket, 316 grade stainless steel

12. Custom cylindrical stainless steel 316 grade bracket

13. $75 \mathrm{~mm}$ memory foam floor, lined with fabric sleeve

14. $75 \mathrm{~mm}$ cross-linked polyethylene, ribbed profile

15. M8x100mm Hex bolt, 316 grade stainless steel

16. Custom floor extrusion, 316 grade stianless steel

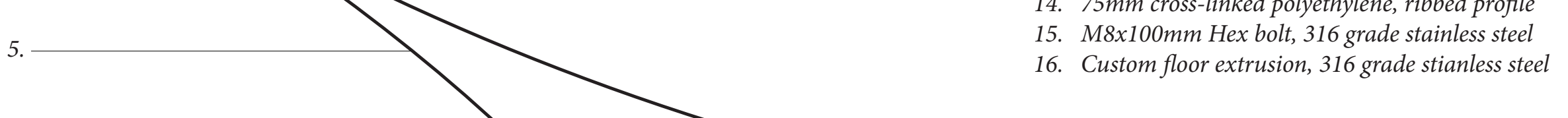

13.

14.

15.
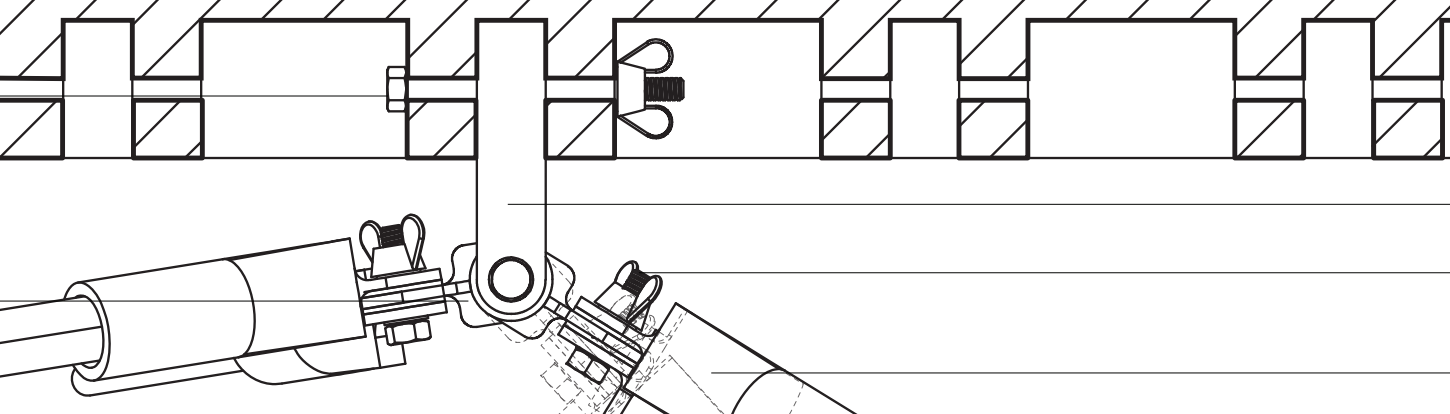

3.

1.

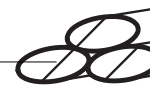

2)

Figure 5.39

Detail Two - Section 1:5

Weave structure fixing to floor surface and membrane at bottom edge. 


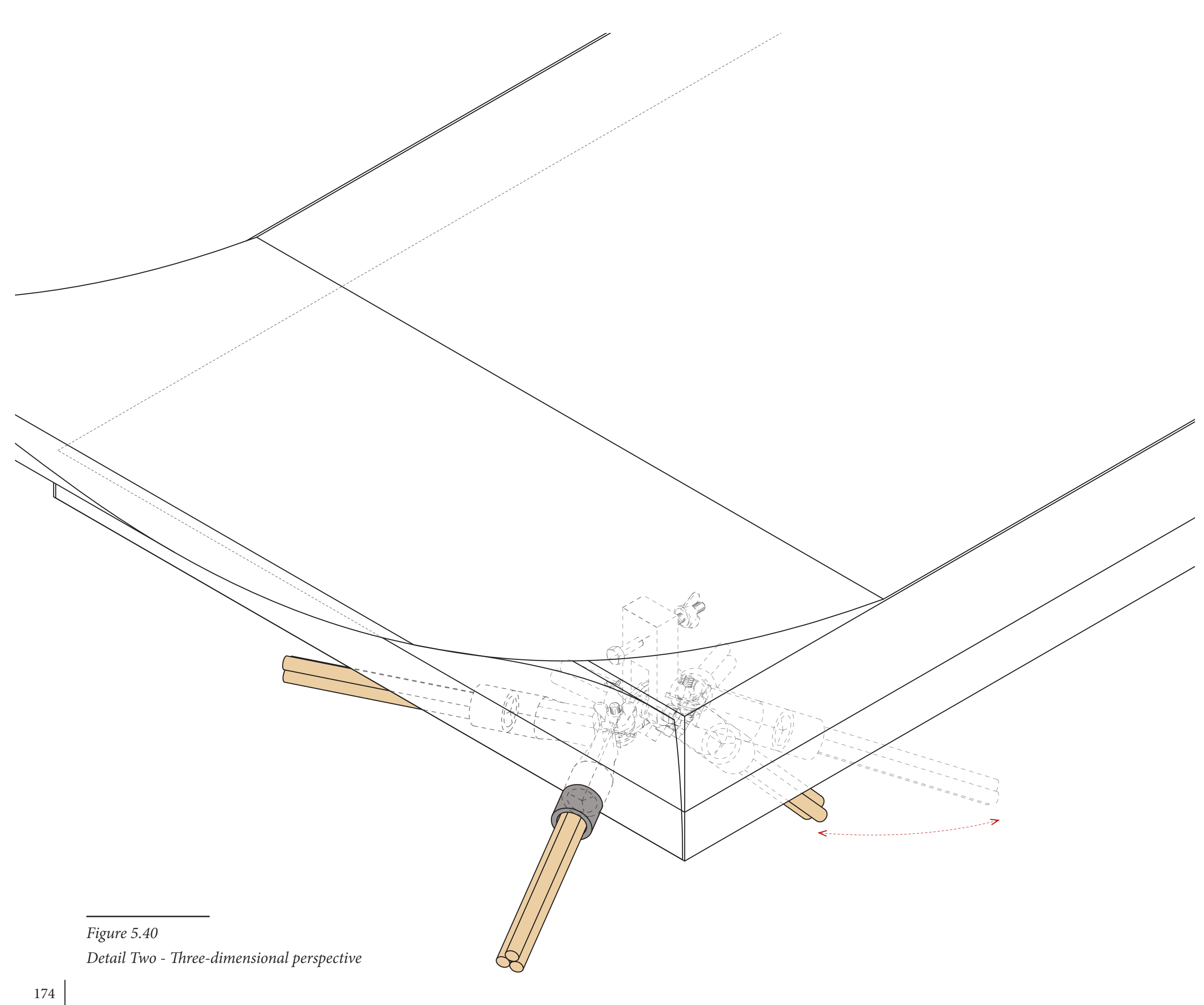




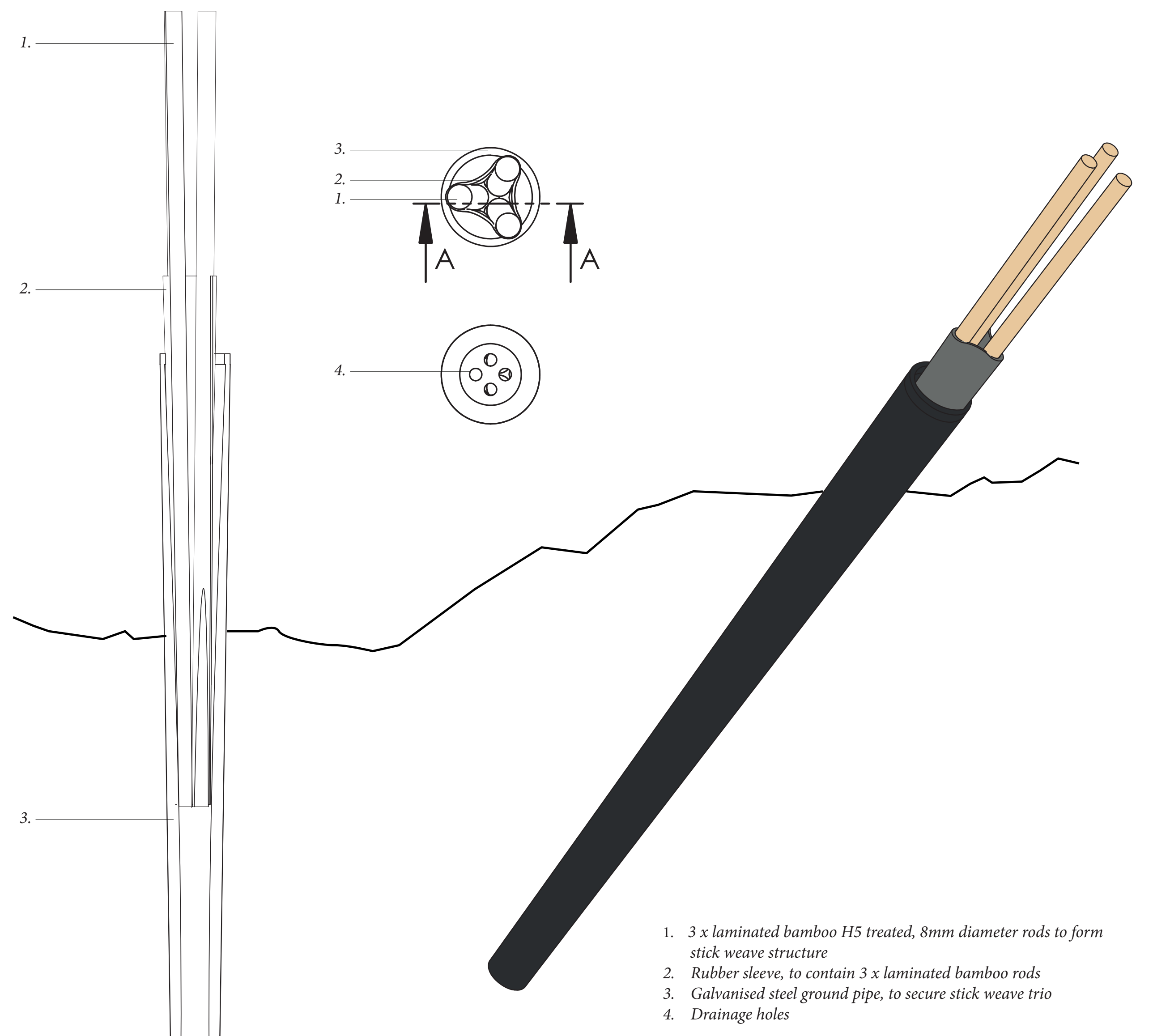

Figure 5.41

Detail Three - Section AA, Top Plan, Base Plan,

Figure 5.42 Three-dimensional perspective 

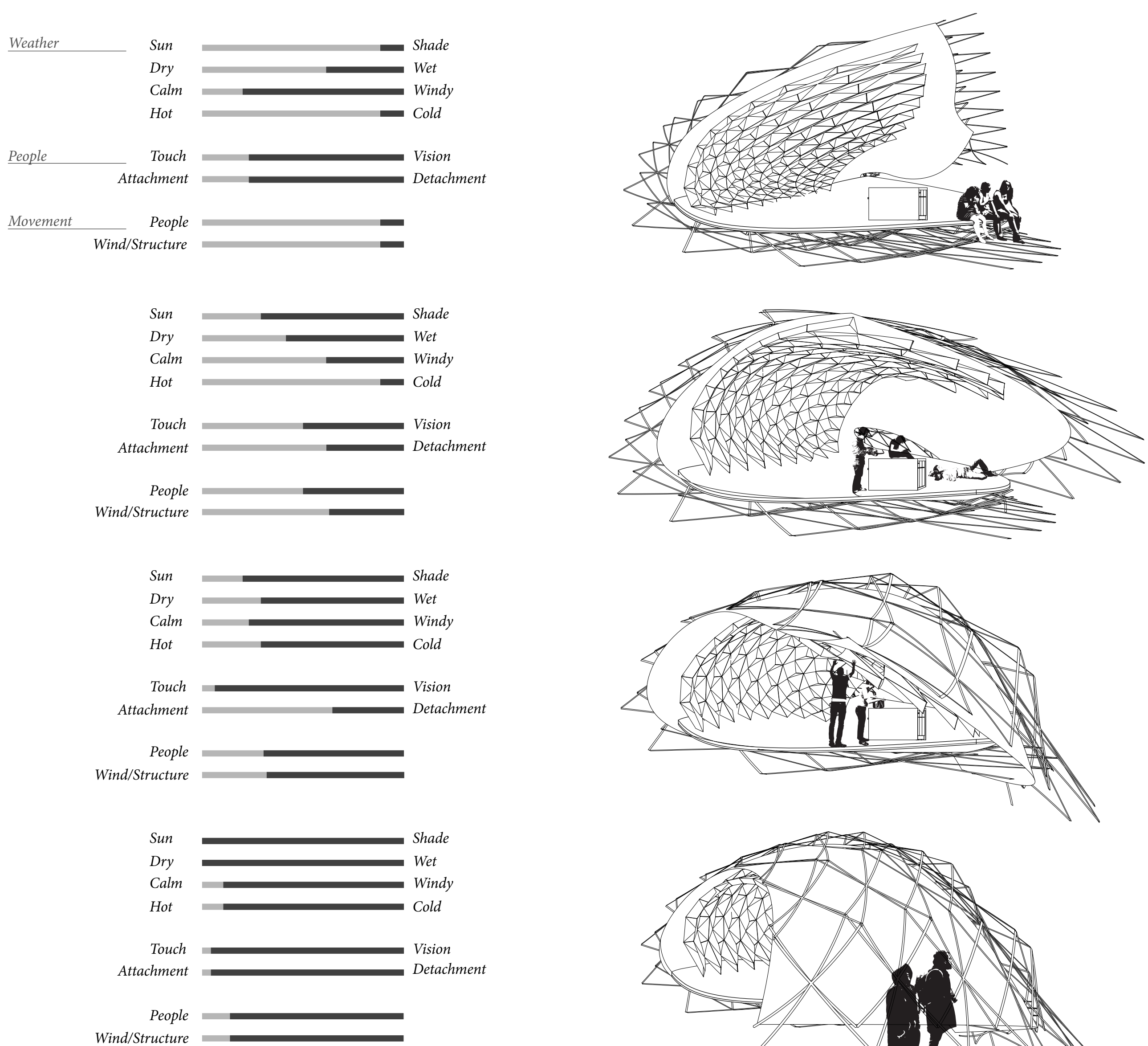

\section{Figure 5.43}

This series of elevations explore occupation of the kitchen as it transforms

through people and wind interaction. The bar sliders define the relationship each phase of form has with the environment, people and movement.

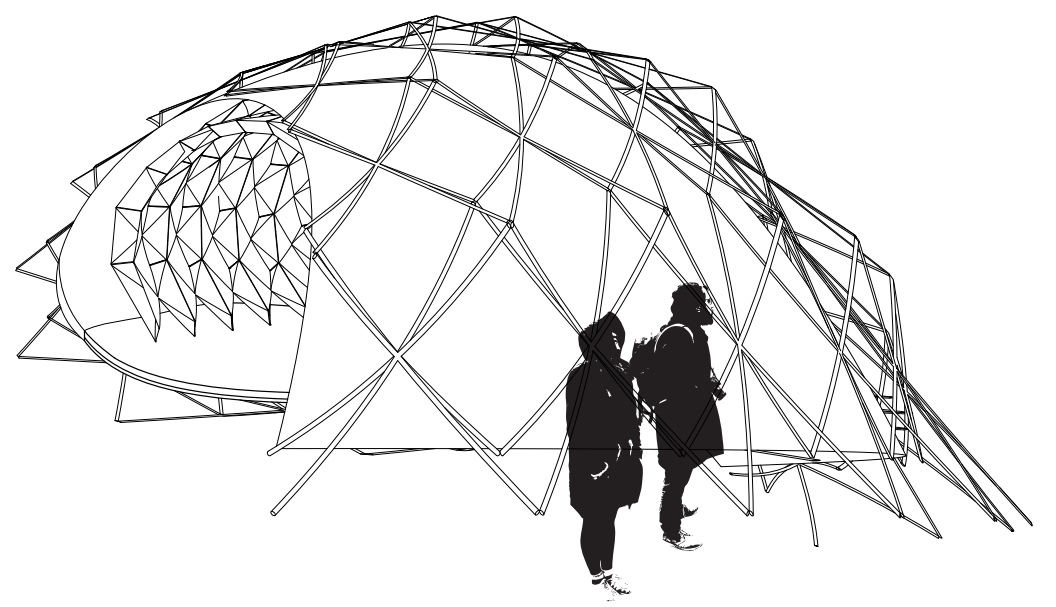



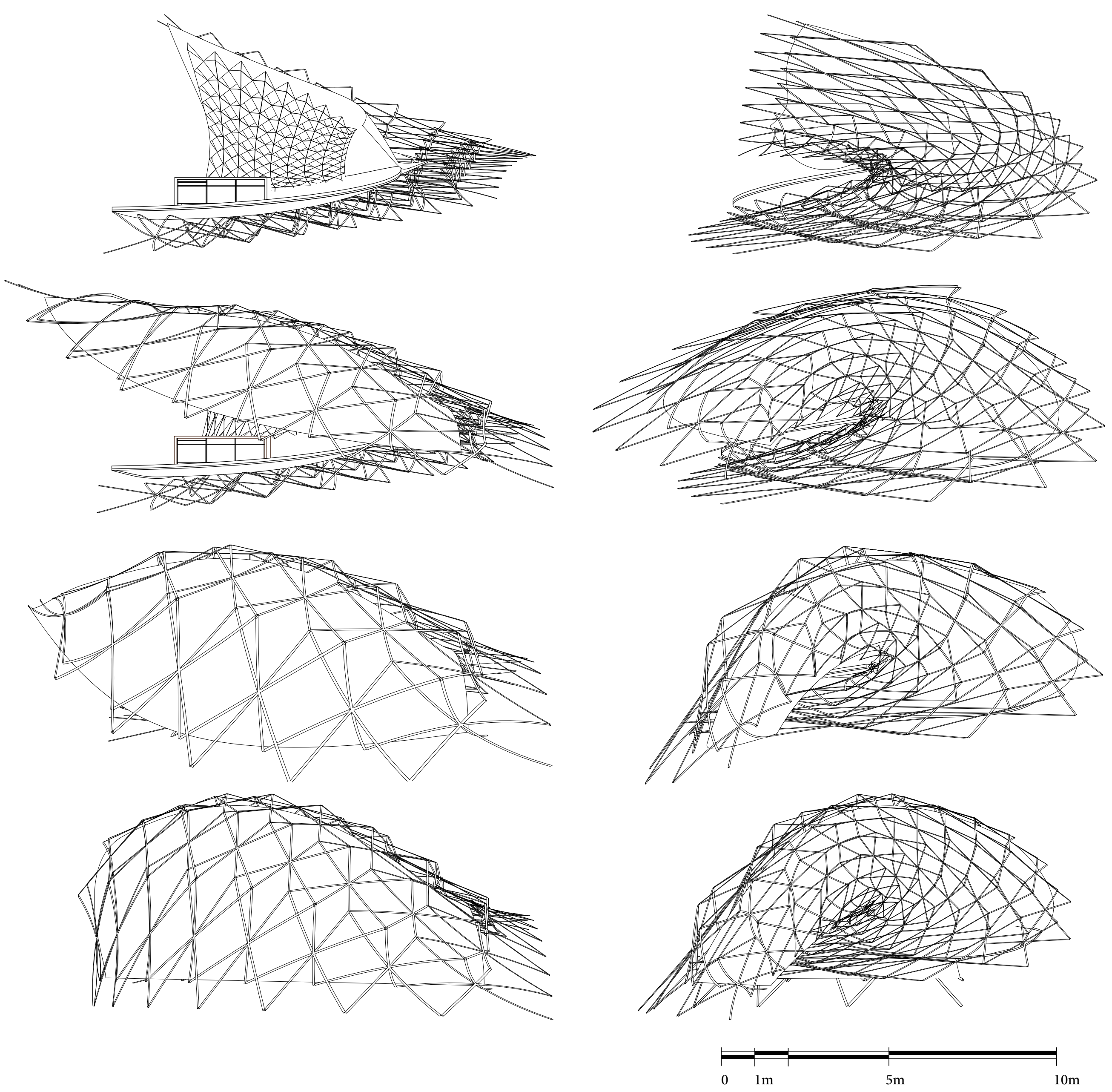


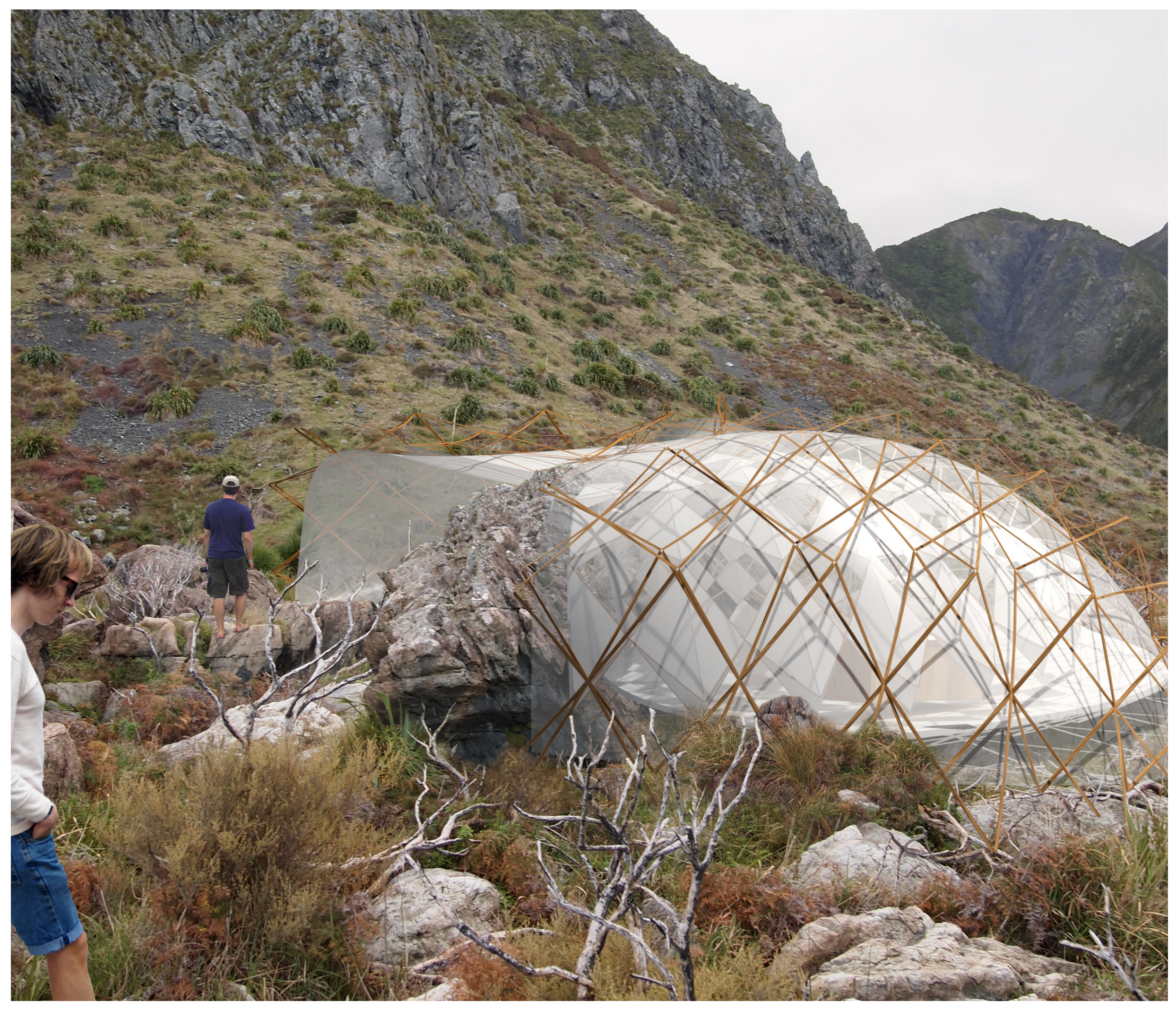

Figure 5.44 Closed Kitchen-Perspective (c) (displayed on Floor Plan) Each of the following four perspectives show the kitchen as it transforms through four stages of inhabitation within a sited context. This closed form is protective at night, and in unpleasant weather conditions. 


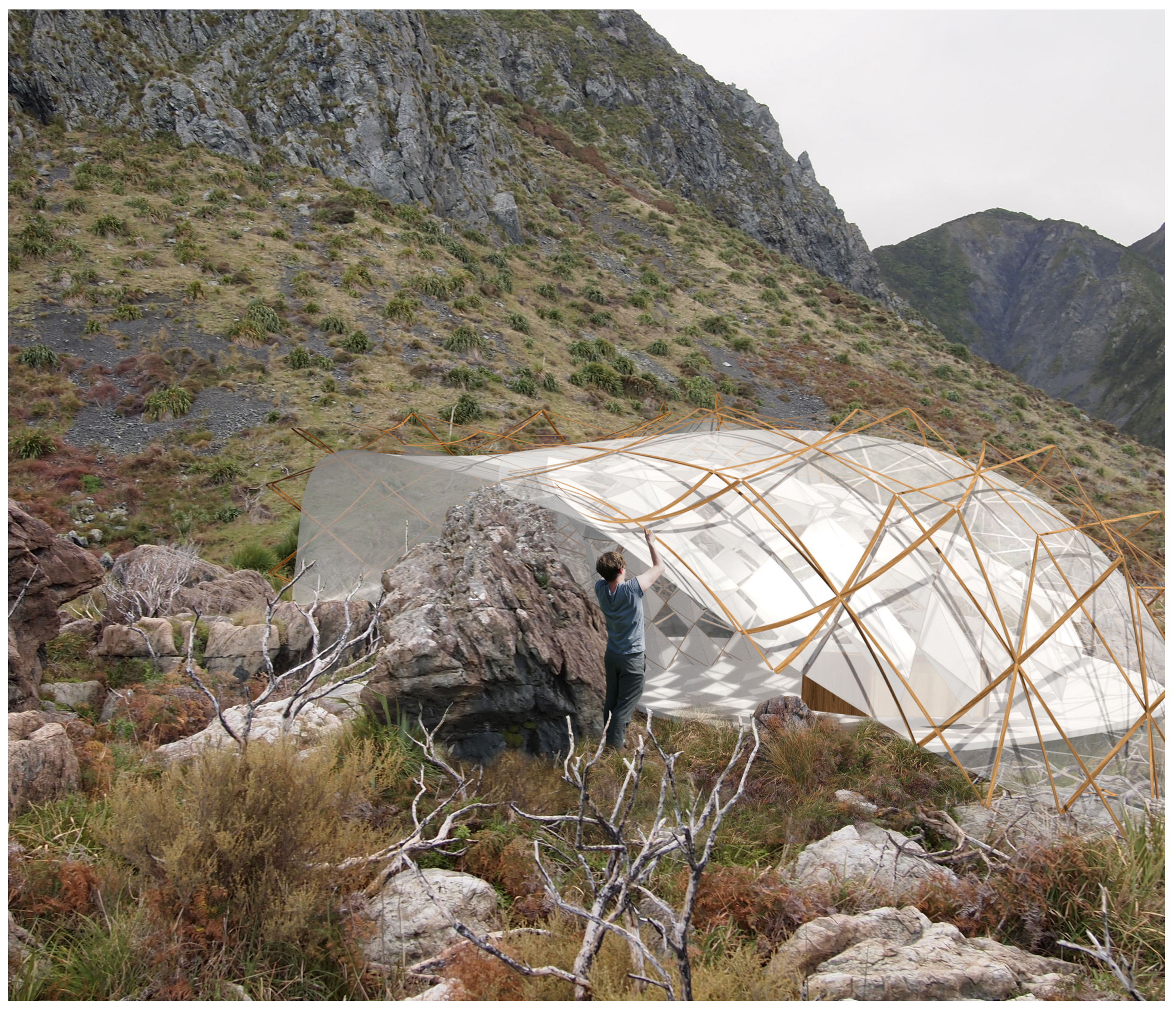

Figure 5.45 Entering Kitchen-Perspective (c)

When closed, to enter each of the shelters the occupant must lift a stick weave from the ground pipe. 


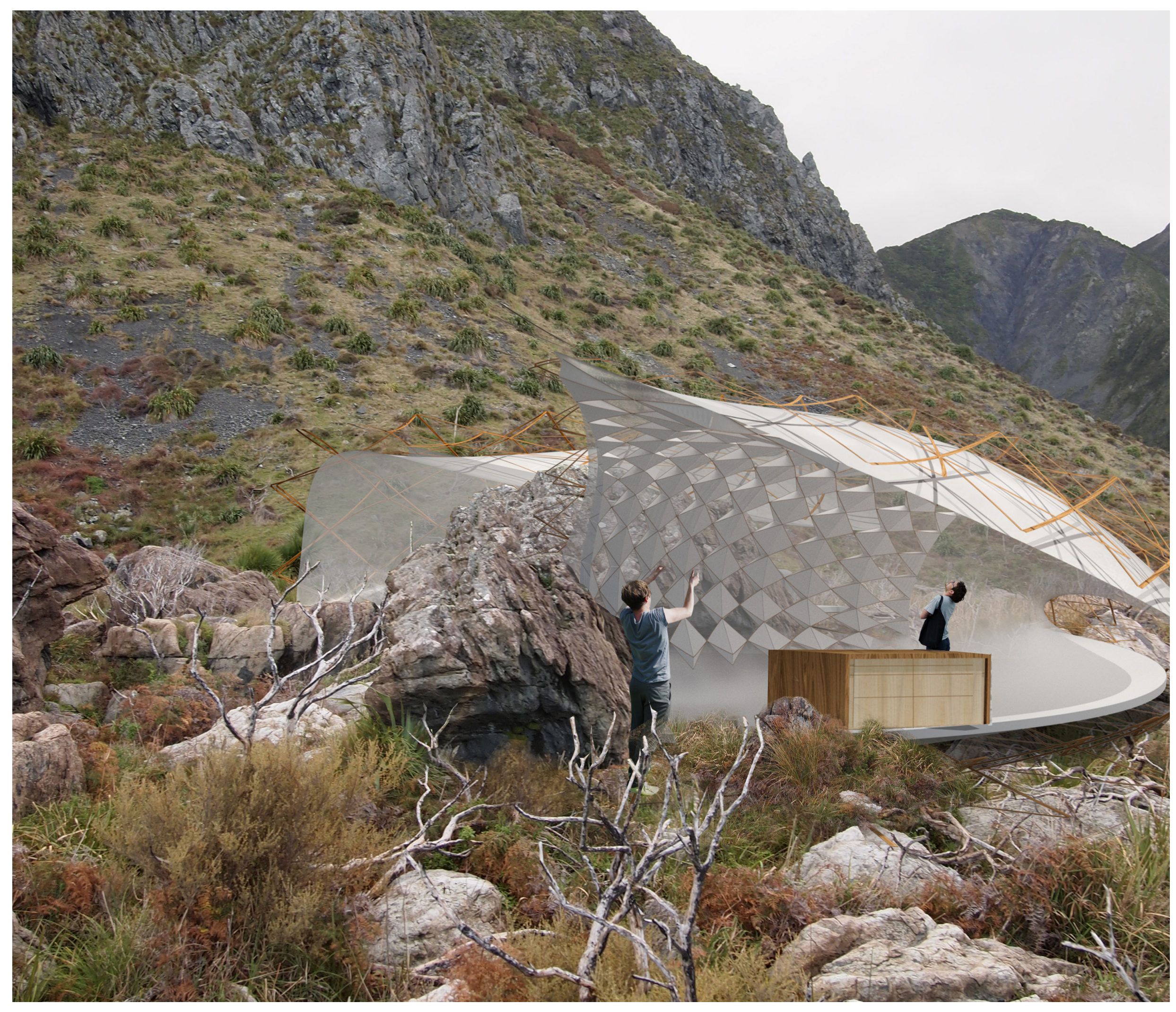

Figure 5.46 Opening + Wind-Perspective (c)

As the structure is gradually released from the ground fixings it is able to

move more freely and interact with wind and other conditions on site. The

openness increases light and ventilation but maintains the shade to make 


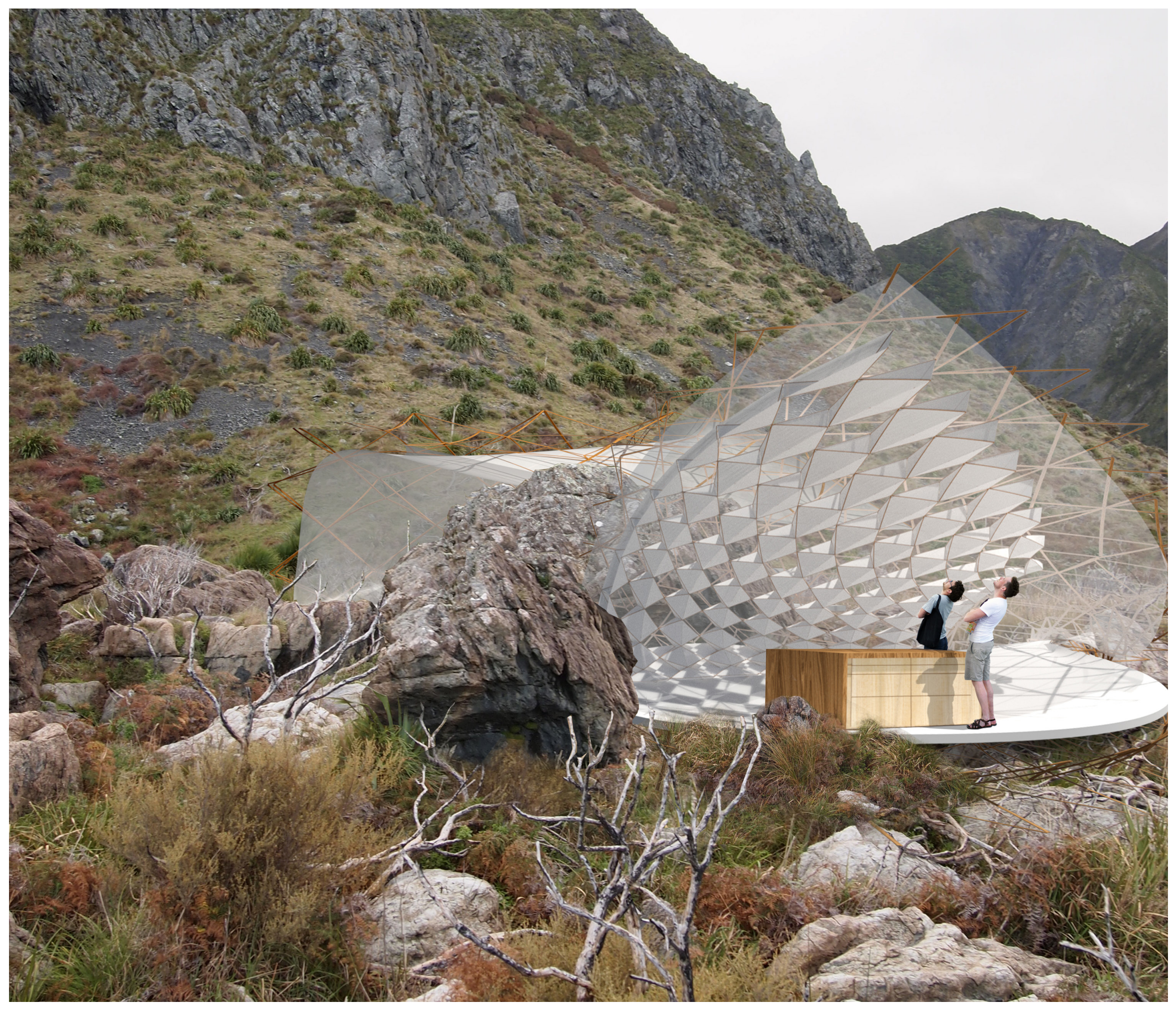

Figure 5.47 Open + Wind-Perspective (c)

The form is now completely open, allowing sun to enter the space and

removing defined interior-exterior boundaries. 


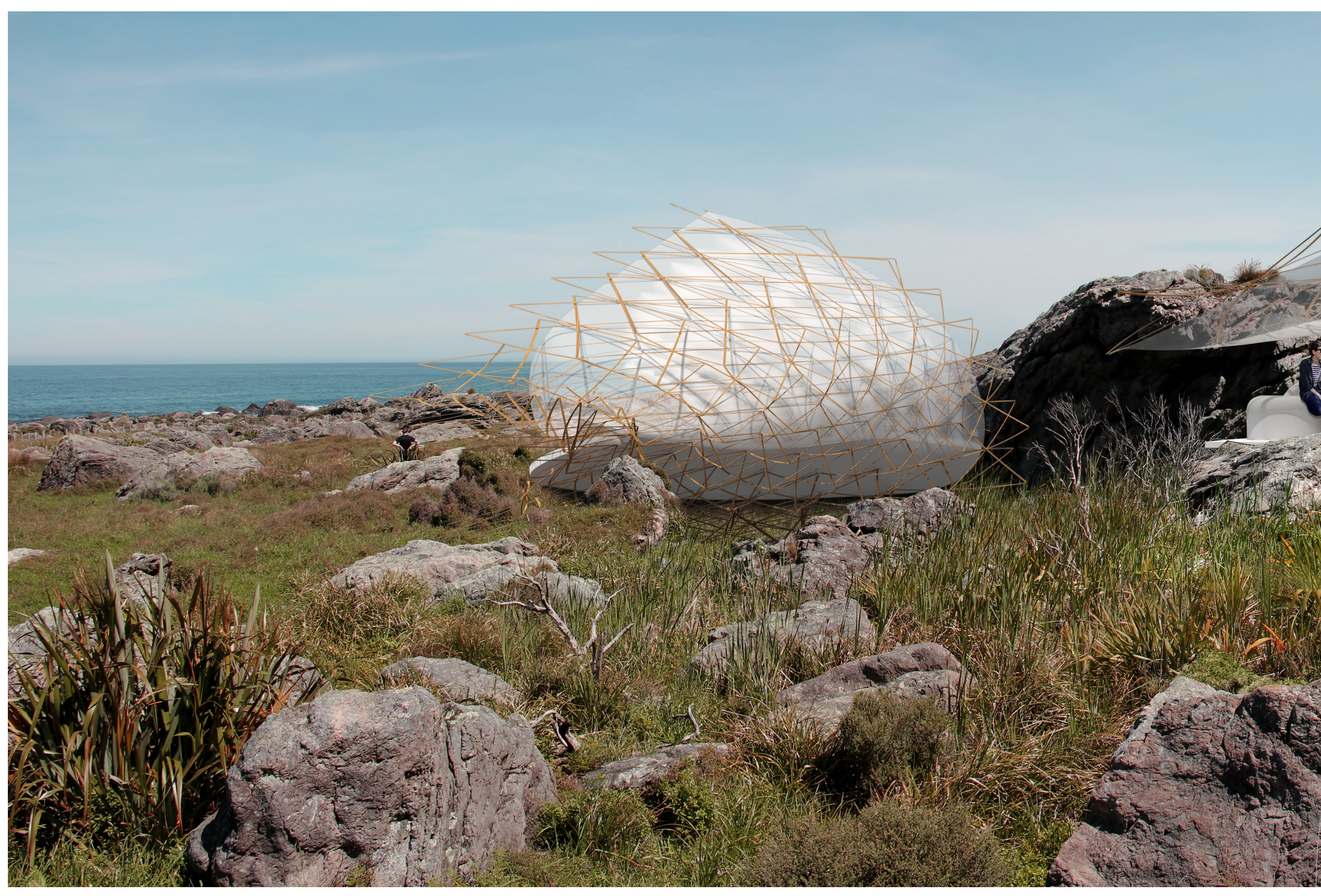

Figure 5.48 Perspective (d), (position indicated on Floor Plan)

Perspective looking south past a double bedroom towards the living-dining and kitchen shelters. 


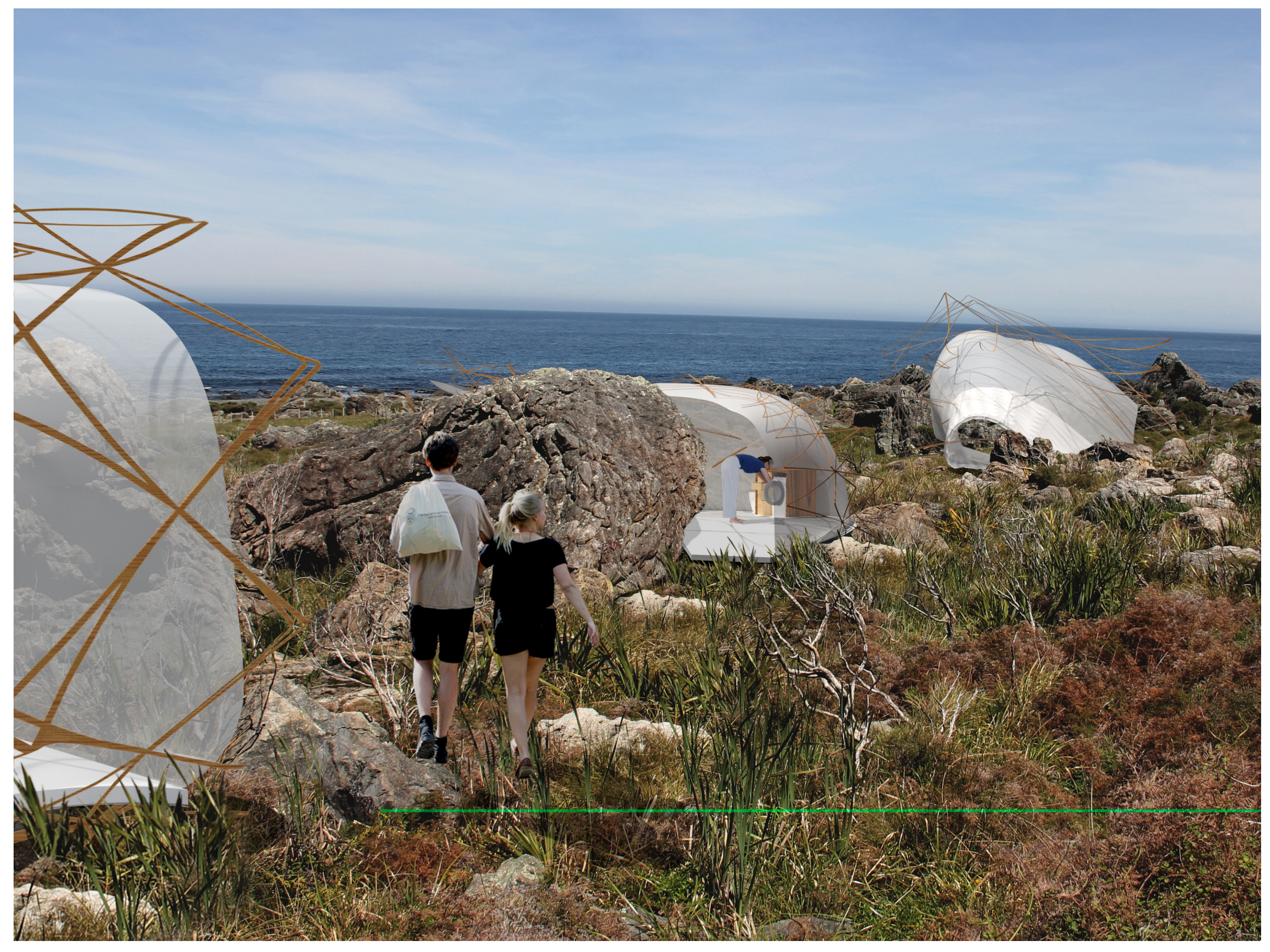

Figure 5.49 Perspective (e)

Walking within the shelters and boulders looking towards the bathroom-

laundry and single bed shelter. 


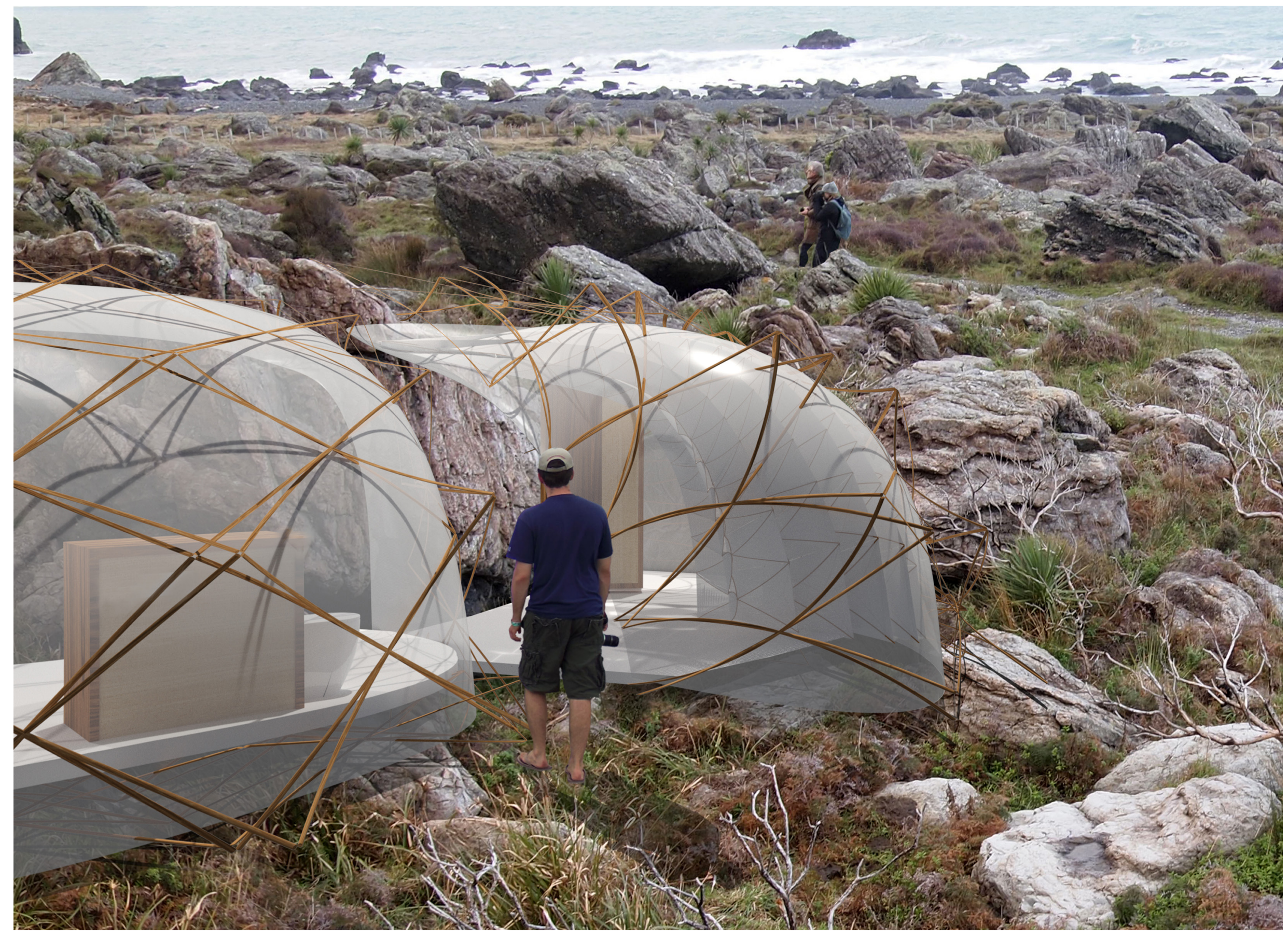

Figure 5.50 Perspective ( $f$ )

Walking towards the shower shelter, past the bathroom-laundry shelter. 


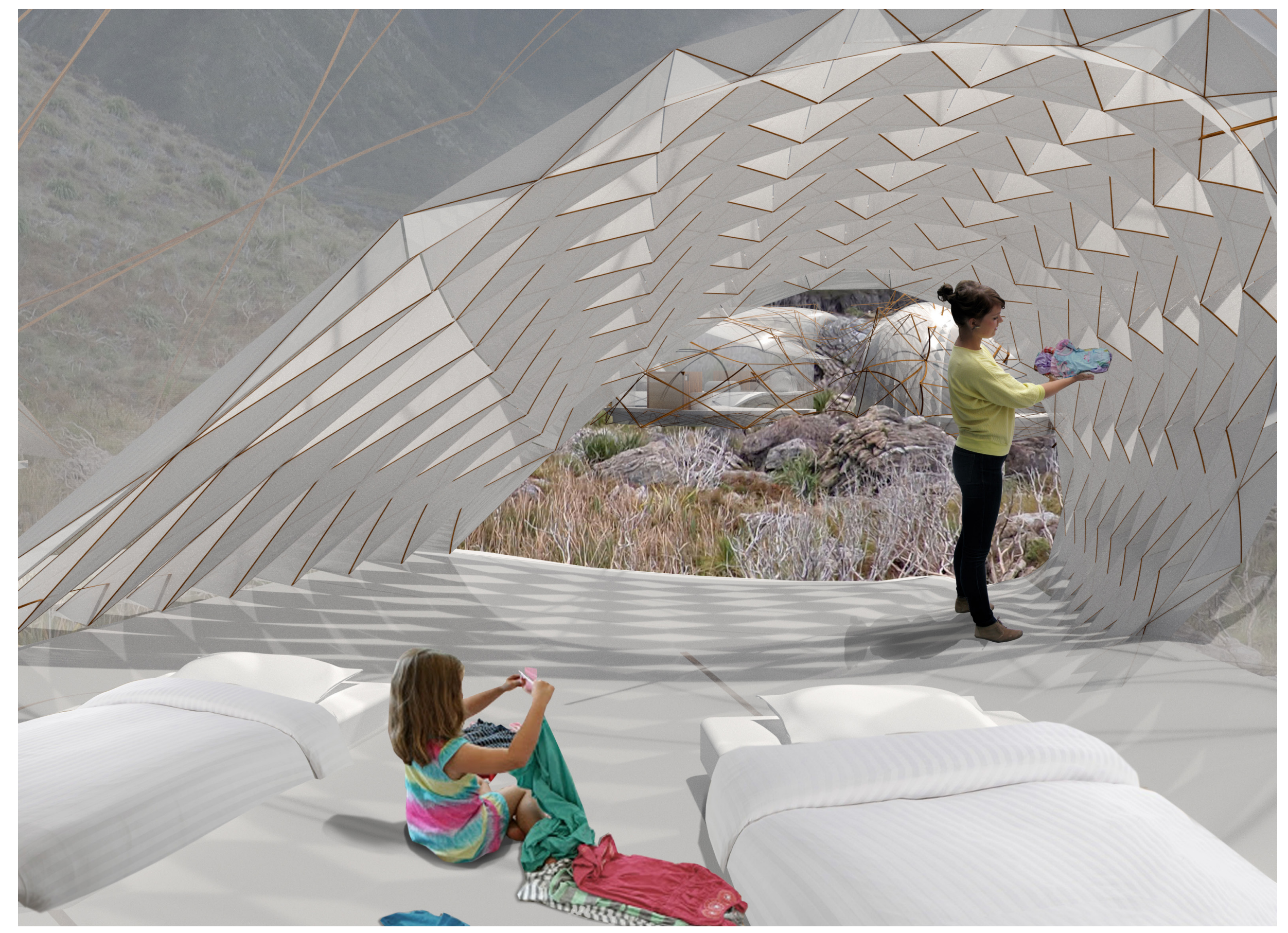

Figure 5.51 Perspective ( $g$ )

Single bedroom perspective displaying inhabitation of the floor plane and use of the storage weave. 


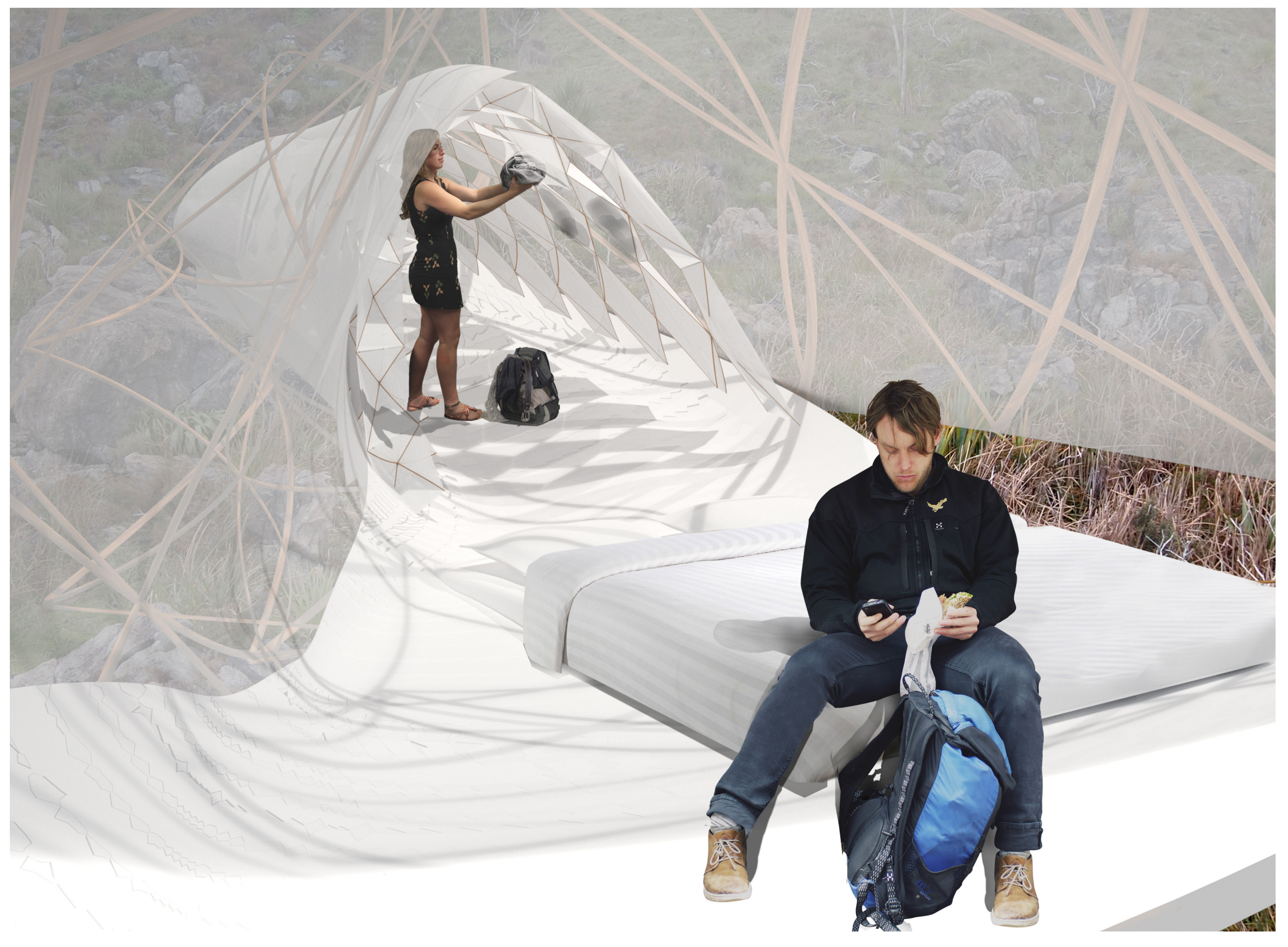

Figure 5.52 Perspective ( $h$ )

Perspective looking into the second double bedroom shelter. 


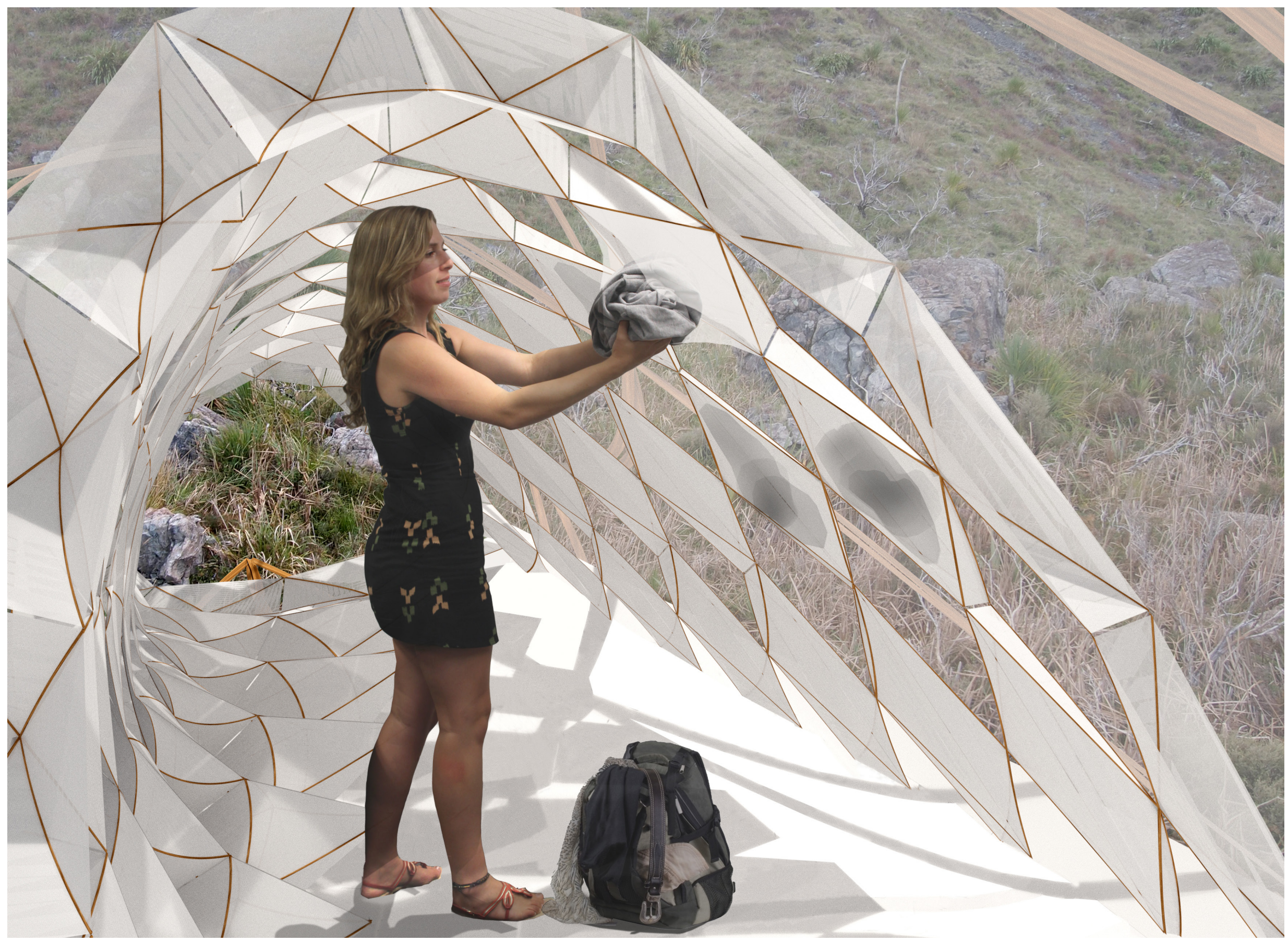

Figure 5.53 Perspective ( $h$ )

Double Bedroom close-up of storage weave.

188 


\section{Phase Three Reflection}

The process for phase three challenges and investigates the differences between digital and analogue design tools and processes. While hand drawings and physical modelling allow rapid prototyping and fast development of form and programme, this was not suited to the desire to reduce architect control. I aimed to create a building that surpasses expectations of form through allowing wind and occupants to alter the shelter. The constant interplay between controllable and uncontrollable influences both survival drives and emotional drives.

The digital realm creates a series of iterations that push the design into different forms and widen the scope of expectation I personally had of the architecture. This in turn re-informed the physical process of prototyping to insure that only the minimum constraints were satisfied.

The differences between digital and analogue design tools specifically related to the generation of affect as a part of the process of design. My digital modelling process successfully simulated the uncontrollable wind force, explored movement and form and synthesising the programme. Despite this, the act of designing through the computer detaches me as the architect from the physical form. The physical modelling process creates attachment and affect through interaction. As the models are physically manipulated the tactile connection with changing forms creates an experience. Affect therefore became a constant gauge of the success of the process as well as an aim for the final design.
The design acts as a work of juxtaposition to emphasise key relationships present. The scales of change within the landscape are referenced through the architectural interaction between fluid elements and permanent insertions into natural features such as the boulders. The sensitive debate regarding wilderness and how culture can be recognised as a part of the landscape to increase its value lies in prioritising the experiential before functional and sculptural as architectural. This same movement also heightens awareness of the relationship between functional and experiential, architecture and sculpture.

\section{References}

1. Standards New Zealand, Structural Design Actions; Part Two: Wind Actions, NZS, 2002. 
190 | 


\section{DISCUSSION}

In this section I discuss the outcomes of this design research investigation with critical focus on the interactive relationships between nature, architecture and occupation. The final shelter design is recognised as only one response to the potential for a beneficial design dialogue with the New Zealand public and the wilderness environment. Interactive architecture is a field that is constantly developing for pragmatic reasons but the focus on experience, learning, connection and awareness of the surrounding context is equally important. The intertwined development of culture and nature is paramount. A final critique considers the value of this investigation within the architectural discipline and the scope for more research to further this

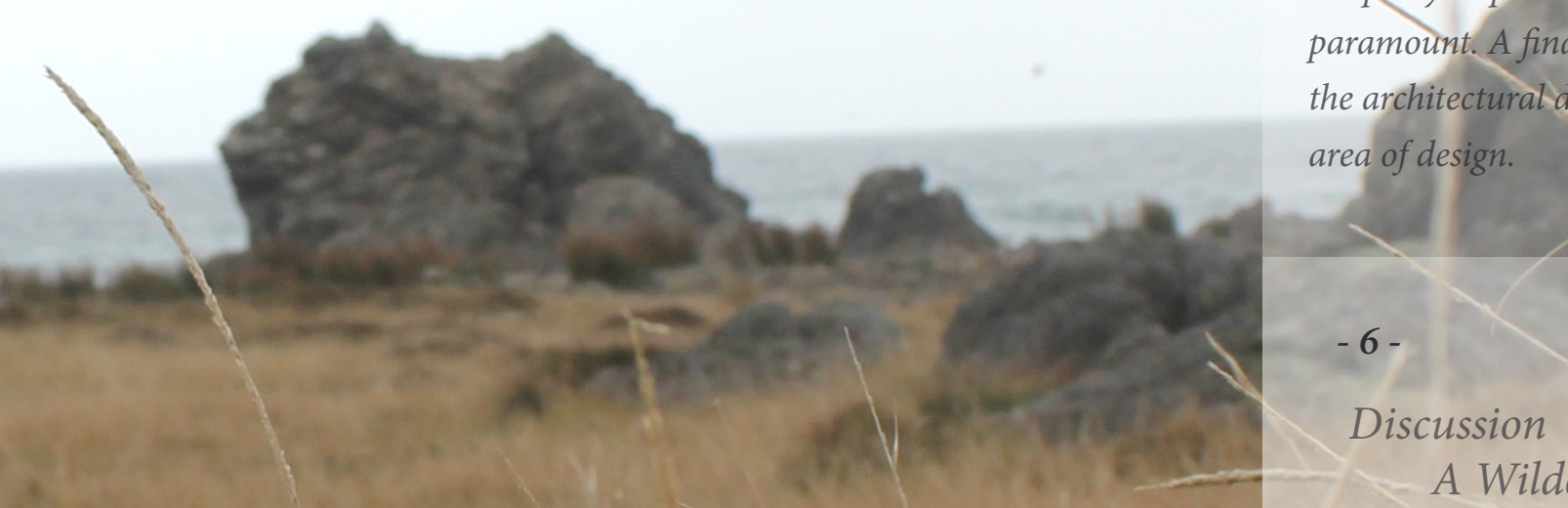

A Wilderness Design Dialogue Reflection 
The notion of shelter is a division between interior and exterior, a split between areas of exposure and areas of protection. This glamping shelter provides a spatial experience that contradicts and questions this distinction. The outcome is a speculative solution that engages the notion of movement and change to increase a person's awareness and connection to wilderness environments and architecture.

Abbott reinforces that the opposition between wilderness and culture is embedded in people's understanding of nature and limits the connection with New Zealand's iconic landscapes. He proposes that the potential for a new public approach to 'landscape' is held within the design discipline as through landscape architecture and architecture the natural environment can become less isolated, more accessible and understood as a vital part of our wellbeing. In response to this, the design proposal is positioned within the 'wilderness' context. It is speculated that a glamorous camping shelter located within the most isolated and extreme landscapes redefines the relationship between wilderness and culture. A shelter can act as a filter, a transition space that introduces and connects people to the ecology, biology and captivating temporality of the wild.

Movement and change, two inseparable concepts are the critical drivers to create a heightened experience of place through dwelling. It is important to note that this key idea is present in two fields; maximising affect and temporality of wilderness environments, but unexplored physically or connected to one-another. This approach to shelter is a tentative exploration into architecture with equal external and internal focus to increase a haptic connection with wilderness.
Temporality is further explored through the notion of permanence. This semi-permanent shelter sits between two wilderness architectural typologies; the backcountry hut, and the tent. One is a permanent addition to the landscape to provide shelter, warmth, and comfort. The other is a semipermanent shelter that offers the same but with a lower level of comfort. Both are successful but the semi-permanent shelter, as with this glamping design, offers a responsive relationship to the surroundings. The different scales of permanence present with these two architectural typologies are likewise present in the wilderness environment, with multiple temporal scales acting in unison. Geological, seasonal, and daily scales of change all alter the feel of the environment. The shelter responds to the temporal scales of change on site through careful detailing from maximising fluid people movements, to wind-driven temporal structure movements, more permanent floors and utilities pods positioned and removed and then the most permanent cuts into rock. Once removed only specific remnants will be left to show that site was once occupied. In this way, the shelter continues to extend the current wilderness architecture typologies by differentiating itself through connection to site as it responds to multiple temporal scales simultaneously.

Connection was investigated through the field of affect as one theoretical approach to understanding the levels of experience. Two different translations of affect; Affect drive theory and Spinozian/Deleuzian theory formed a framework for designing the shelter. The critique of Freudian's model of affective drives by Tomkins, and Sedgwick and Frank, forms an understanding of the relationship between survival time-constrained drives; eating, sleeping, drinking and experiential or emotional drives and have no time-limit. The shelter was initially designed to satisfy both drives

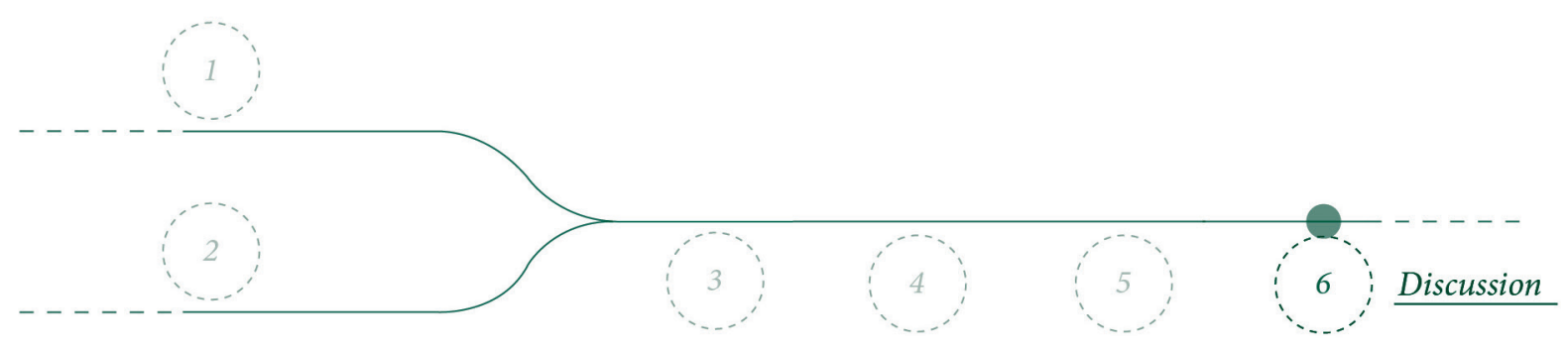


as separate elements of experience with one canopy sheltering all of the programme. A shift in approach isolated each function and created areas of the design where the stick weave is completely inhabited, such as the bedrooms, so the spaces are designed to satisfy both drives not separately but in unison. Functional spaces are juxtaposed with fluid structure and people movement to maintain a constant interplay between controllable and uncontrollable interaction with the architectural space.

Nigel Thrift who writes on the Spinozian/Deleuzian approach to affect theory proposes a series of viewpoints on how the experience of affect can be increased. These methods of tending and manipulation of space and time are investigated in the shelter through the key idea of movement and change. Initially this design research began as a formal exploration of archetypal norms; traditional roof, walls and floor. This was decomposed into a design that borders fields of art, sculpture and landscape architecture. The transformable spatial design relies on the structures physical transformations to create moments of forced interaction and realisation. As the shelter moves, the constant and often unrealised relationship between a building and its surroundings are emphasised. Movement is synonymous with change and this relates to the notion of tending. Therefore, this design proposes that the temporality of the environment is not excluded through a clear division of exterior and interior, but is included to create a constantly changing spatial experience. A nontraditional approach to roof, walls and floors through one fluid structure removes social expectations of space and creates room for a person to have a new experience and form new connections with their surroundings.

This tentative design solution leaves room for further theoretical investigation into the relationship between inhabitation of space, and non-static architecture. How can movement alter routine and expectations of space, and does this responsive interactive design have a place in the wider architectural field. The shelter specifically leaves room for structural development as further prototyping and testing could resolve the structural joints and skin to product level resolution.

It is also important to note that design led research as a process engages with multiple levels of design, theoretical questioning, physical and digital testing, and with addition of practical design constraints creates a repetitive cyclic process. The shelter design process is an investigation of theoretical enquiries that creates a physical connection with me, as the designer through modelling. The act of manipulating models tests the act of occupants inhabiting the shelter to create conclusions that could not be reached through theorising alone. 
${ }^{194}$ | 


\section{CONCLUSION}

This thesis is concluded with a final chapter that summarises the complete outcomes of the design research process. 


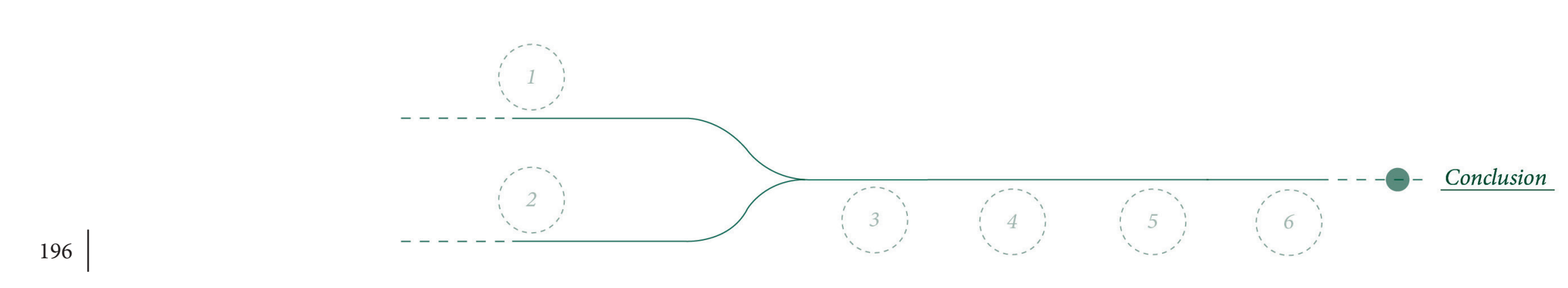


Architecture is designed to resist the temporality of the environment; the cold, wet, heat and darkness. This static fixity of buildings does not interact with the surrounding environment and detaches the occupant from their immediate environment. The aim of this thesis was to explore how non-static architecture that embodies movement could heighten an occupants awareness of both architecture and environment. A theoretical understanding of affect provides a framework that permeates the design process to generate an affective architecture.

This research is situated within a rich theoretical, artistic and architectural context formed around the areas of wilderness, affect and non-static. It differentiates itself through the investigation of affect through a process of physical modelling to form a built design. Furthermore, the non-static interactive architecture is explored with focus on attachment and experience as opposed to technological or spatial development for comfort or efficiency. Finally, the idea of a remote, isolated wilderness is challenged through the creation of a design dialogue which uses architecture to reinforce an equal nature-culture ideal. The final design sits on the boundary between sculpture, architecture and landscape to create an interactive shelter that challenges preconceived expectations of how wilderness and architecture can come together.

The transformable structure is central to the success of this enquiry. This thesis argues that the experience of being within the wilderness can be dramatized using moving structure to reveal an unrealised but constant interaction between weather conditions, spaces, structure and use. The interplay between functional use of utilities and experiential moving qualities of the design challenges the user to realise how they inhabit space.
The stick weaving system is a complex and intricate solution that is one of many possible approaches to changing form. While complete resolution of the system is outside the scope of this thesis, structural movement still holds true as a valid method to enhance a person's connection to architecture and environment.

The design led research approach had agency in understanding how analogue and digital design processes differed. Although with collaboration, the process could reduce designer control and create a building which form is controlled by weather and occupation. Every aspect of the building is designed to embody a sense of movement. The careful choice of siting, materiality, spatial configuration, programme and most of all structural system maximises the movement within and around the shelters. The fluidity of the form removes interior-exterior boundaries and creates a seamless inhabitation of architecture and environment as one. 
${ }_{198} \mid$ 
WORKS CITED 
$24 H>$ architecture. "Ecological Children Activity and Education Center / $24 H>$ Architecture." ArchDaily, 2007 2006. http://www.archdaily. com/34946/ecological-children-activity-and-education-center-24harchitecture/.

Abbott, Mick. "Designing Wilderness as a Phenomenological Landscape: Design-Directed Research Within the Context of New Zealand's Conservation Estate." Lincoln University, 2008. http:// researcharchive.lincoln.ac.nz/dspace/handle/10182/1026.

Agkathidis, Asterios, Johan Bettum, Markus Hudert, and Harald Kloft. Digital Manufacturing in Design and Architecture. Amsterdam: BIS Publishers, 2010 .

Architecture Uncomfortable Workshop. "Spring Wind House." Designboom. Accessed March 24, 2013. http://www.designboom.com/readers/ spring-wind-house/.

ART+COM. "ART+COM : Manta Rhei." Accessed March 25, 2013. http:// www.artcom.de/en/projects/project/detail/manta-rhei/.

"Kinetic Rain" Changi Airport Singapore, 2012. http://vimeo. com/45188800.

Beardsley, John. "Kiss Nature Goodbye. Marketing the Great Outdoors." Harvard Design Magazine 10 (2000): 1-6.

Bhatt, Mehul, Frank Dylla, and Joana Hois. "Spatia-Terminological Inference for the Design of Ambient Environments." In Spatial Information Theory, 371-391. Aber Wrac'h, France: COSIT, 2009.

"Black Beech." NZ Wood. Accessed November 12, 2013. http://www. nzwood.co.nz/learning-centre/black-beech/.

Blake, David. "Assorted Wilderness Photography." Google+, January 19, 2010. https://plus.google.com/photos/115458569418877025347/alb ums/5430113215124574049?banner=pwaepartnerid=pwrd1.

Boffa Miskell. Wairarapa Landscape Study 2010; Landscape Character Description Report. Greater Wellington Regional Council, August 2010.

Bradford Hansen-Smith. "The History of Stickweaving." Stickweaving: The
Work of Bradford Hansen-Smith, 2007. http://www.stickweaving. com/history.html.

Braun, Bruce, and Noel Castree. Remaking Reality: Nature at the Millenium. Routledge, 1998.

Brooker, Edward, and Marion Joppe. "Trends in Camping and Outdoor hospitality - An International Review." Journal of Outdoor Recreation and Tourism 3-4 (December 2013): 1-6.

Bruce, A. P. Indigenous Vascular Plants in the Vicinity of Cape Palliser, Southern Wairarapa, S.L.-1000Ft. (Ngawhi to Ngapotiki - N 168/9). Wairarapa: Department of Scientific and Industrial Research, Botany Division, 1987.

Bultena, Gordon, and Marvin J. Taves. "Tenting on a Park Campground." Conserv. Volunteer 23 (1960): 60-64.

City Form Lab. "SUTD Library Pavilion." ArchDaily, June 17, 2013. http:// www.archdaily.com/387696/sutd-library-pavilion-city-form-lab/.

Corner, James. Recovering Landscape: Essays in Contemporary Landscape Theory. Princeton Architectural Press, 1999.

Cronon, William. "The Trouble with Wilderness: Or, Getting back to the Wrong Nature." Environmental History 1, no. 1 (1996): 7-28.

Cullen, Ross, Jim Harland, Craig Potton, and New Zealand Tourism Policy Group. Collection of Essays on Equity and Access to Natural Areas. Tourism Policy Group, Ministry of Commerce, 1994.

Digital Architectural Lab. "DAL Canopy Design." ArchDaily, September 1, 2011. http://www.archdaily.com/165298/dal-canopy-design-digitalarchitectural-lab/.

Dolson, Bill. "Landscape Temporal Variations." Bill Dolson, February 24, 2007. http://www.billdolson.com/Variations/variations.htm.

El-Khoury, Rodolphe, Christos Marcopoulos, and Carol Moukheiber. The Living, Breathing, Thinking, Responsive Buildings of the Future. London: Thames \& Hudson, 2012.

Fox, Michael, and Miles Kemp. Interactive Architecture. Princeton 
Architectural Press, 2009.

Franz, Jill. "The Potential of the Window in 'Framing' Landscape Meaning." IDEA Journal (2005): 85-95.

Harris, Graham. "Maori Land Restoration: The Matakitaki-a-Kupe Project." Accessed April 27, 2013. http://www.rnzih.org.nz/pages/NZ-Plantsand-their-Story-45-50.pdf.

"Rengarenga Lilies And Maori Occupation At Matakitaki-a-Kupe (Cape Palliser): An Ethnobotanical Study, By Graham F. Harris And Haami Te Whaiti, P 271-286." Journal of the Polynesian Society 105, no. 3 (1996): 271-286.

Higgleton, Elaine, Howard Sargeant, and Anne Seaton, eds. Chambers Pocket Dictionary. Edinburgh: Chambers Harrap Publishers, 1997.

"Hinau." NZ Wood. Accessed November 12, 2013. http://www.nzwood. co.nz/learning-centre/hinau/.

Hui, Wing Yi, and Lap Ming Wong. "Wood Pavilion." ArchDaily, July 12, 2010. http://www.archdaily.com/68446/wood-pavilion-wing-yihui-lap-ming-wong/.

Hull, R. Bruce, David P. Robertson, and Angelina Kendra. "Public Understandings of Nature: A Case Study of Local Knowledge about Natural' Forest Conditions." Society \& Natural Resources 14, no. 4 (2001): 325-340

Jansen, Theo. "Strandbeest." Theo Jansen's Strandbeest. Accessed January 10, 2014. http://www.strandbeest.com/theo_cv.php.

Karawitz Architecture. "Passive House." ArchDaily, 2009. http://www. archdaily.com/84165/passive-house-karawitz-architecture/.

Karjalainen, Eeva, Tytti Sarjala, and Hannu Raitio. "Promoting Human Health through Forests: Overview and Major Challenges." Environmental Health and Preventive Medicine 15, no. 1 (March 25, 2009): $1-8$

Keskisarja, Emmi, Pekka Tynkkynen, and LEAD. "Dragon Skin Pavilion." ArchDaily, March 10, 2012. http://www.archdaily.com/215249/ dragon-skin-pavilion-emmi-keskisarja-pekka-tynkkynen-lead/.
Klatzky, Roberta L., and Susan J. Lederman. "Haptic Object Perception: Spatial Dimensionality and Relation to Vision." Philosophical Transactions of the Royal Society B: Biological Sciences 366, no. 1581 (November 12, 2011): 3097-3105.

Kundig, Tom. "Delta Shelter." Olson Kundig Architects, 2013. http://www. olsonkundigarchitects.com/Projects/38/Delta-Shelter.

"Rolling Huts." Olson Kundig Architects, 2013. http://www. olsonkundigarchitects.com/Projects/825/Rolling-Huts.

Leach, B. Foss. "The Prehistory of the Southern Wairarapa." Journal of the Royal Society of New Zealand 11, no. 1 (1981): 11-33.

Macnaghten, Phil, and John Urry. Contested Natures. Vol. 54. Sage, 1998.

Mannell, Roger C., and Seppo E. Iso-Ahola. "Psychological Nature of Leisure and Tourism Experience." Annals of Tourism Research 14 no. 3 (1987): 314-331.

Marks, Kathrin, and Stefan Marks. Sabine Hut, February 8, 2011. http:// www.flickr.com/photos/ks_marks/5468587193/.

Maslow, Abraham Harold, and Karen J. Lewis. Maslow's Hierarchy of Needs. Salenger Incorporated, 1987. http://www. researchhistory. org/2012/06/16/maslows-hierarchy-of-needs/.

Mount Fuji Architects Studio. "Rainy|Sunny / Mount Fuji Architects Studio." ArchDaily, 2008. http://www.archdaily.com/48381/rainy-sunnymount-fuji-architects-studio/.

Murdoch, Jonathan. "Inhuman/nonhuman/human: Actor-Network Theory and the Prospects for a Nondualistic and Symmetrical Perspective on Nature and Society." Environment and Planning D 15 (1997): $731-756$

New Zealand Wood. "Timber Performance and Durability." NZ Wood. Accessed November 12, 2013. http://www.nzwood.co.nz/learningcentre/lc-timber-performance-durability/.

Newton, Bronwyn M., John R. Fairweather, and Simon R. Swaffield. "Public Perceptions of Natural Character in New Zealand: Wild Nature Versus Cultured Nature." New Zealand Geographer 58, no. 2 
(2002): 17-29.

Noble, David. "Travers - Sabine Circuit - Nelson Lakes National Park, Day6." David Noble, January 10, 2010. http://www.david-noble. net/NZ/Jan10/TraversSabine/Day6.html.

Parsa, Pouya Khazaeli. "Bamboo Structure Project." ArchDaily. Accessed June 26, 2013. http://www.archdaily.com/93922/bamboo-structureproject-pouya-khazaeli-parsal.

rAndom. "'Rain Room' Installation / rAndom." ArchDaily, 2012. http:// www.archdaily.com/288219/rain-room-installation-random/.

"Swarm Light - rAndom International," 2010. http://randominternational.com/work/swarm-light/.

"Swarm Study / III - rAndom International," 2011. http://randominternational.com/work/swarm-study-iii/.

"Rewarewa." NZ Wood. Accessed November 12, 2013. http://www.nzwood. co.nz/learning-centre/rewarewa/.

Robertson, Jan. Sabine Hut View Nelson Lakes, December 31, 2010. http:// www.flickr.com/photos/backcountrynz/8609196904/.

Schweitzer, Marc, Laura Gilpin, and Susan Frampton. "Healing Spaces: Elements of Environmental Design That Make an Impact on Health." The Journal of Alternative and Complementary Medicine 10, no. 1 (September 1, 2004): 71-83.

Sedgwick, Eve Kosofsky, and Adam Frank. Touching Feeling: Affect, Pedagogy, Performativity. Duke University Press, 2003

Shouse, Eric. "Feeling, Emotion, Affect." Journal of Media and Culture 8, no. 6 (December 2005). http://www.journal.media-culture.org. au/0512/03-shouse.php.

Shultis, John. "The Duality of Wilderness." The State of Wilderness in New Zealand (2001): 59.

Sowers, Charles. "Charles Sowers | Wave Wall." Charles Sowers Public Art Works and Science Experiments, 2006. http://charlessowers.com/ wave-wall.
“Charles Sowers | Windswept," 2011. http://charlessowers.com/ windswept.

Standards New Zealand. Structural Design Actions; Part Two: Wind Actions. NZS, 2002.

Studio Gang Architects. "Lincoln Park Zoo South Pond." ArchDaily, October 22, 2010. http://www.archdaily.com/83676/lincoln-park-zoo-southpond-studio-gang-architects/.

Tang, Ming. "Folded Bamboo + Paper House." ArchDaily, 2008. http://www. archdaily.com/8124/folded-bamboo-paper-house-ming-tang/.

The British Camping and Caravanning Club. "Get Richer, Feel Better." WebLabs.Modules.ContentPages.IndexPage. The Camping and Caravanning Club, April 15, 2011. http://www. campingandcaravanningclub.co.uk/newsandevents/get-rich-quick/ real-richness-the-list/get-richer-feel-better/.

Theo Jansen - The Great Pretender, 2009. http://www.youtube.com/ watch? $v=5$ NOIonPOy-I\&feature $=y o u t u b e \_g d a t a \_p l a y e r$.

Thrift, Nigel. "Intensities of Feeling: Towards a Spatial Politics of Affect." Geografiska Annaler: Series B, Human Geography 86, no. 1 (2004): $57-78$.

Tomkins, Silvan S. Exploring Affect: The Selected Writings of Silvan S Tomkins. Cambridge: Cambridge University Press, 1995.

Tongji University Team. "O-STRIP Pavilion." ArchDaily, October 6, 2011. http://www.archdaily.com/174127/o-strip-pavilion-tongjiuniversity-team/.

“Totara." NZ Wood. Accessed November 12, 2013. http://www.nzwood. co.nz/learning-centre/totara/.

Trippy Tramper. “Tramping: New Angelus Hut,” October 31, 2010. http://rippytramping.blogspot.co.nz/2010/11/new-angelushut-30-31102010.html.

University of Stuttgart, Faculty of Architecture and Urban Planning. "ICD/ ITKE Research Pavilion." ArchDaily, March 6, 2013. http://www. archdaily.com/340374/icditke-research-pavilion-university-of- 
stuttgart-faculty-of-architecture-and-urban-planning/.

Vo Trong Nghia. "Bamboo Wing." ArchDaily, 2010. http://www.archdaily. com/219880/bamboo-wing-vo-trong-nghia/.

Vries, Sjerp de, Robert A Verheij, Peter P Groenewegen, and Peter Spreeuwenberg. "Natural Environments, Healthy Environments? An Exploratory Analysis of the Relationship between Greenspace and Health." Environment and Planning A 35, no. 10 (2003): 1717 1731. doi:10.1068/a35111.

White, Naomi Rosh, and Peter B. White. "Travel as Transition: Identity and Place." Annals of Tourism Research 31, no. 1 (January 2004): 200-218. doi:10.1016/j.annals.2003.10.005.

Zamorano, Pablo Esteban, Nacho Martí, and Jacob Bek. "Expandable Surface Pavilion." ArchDaily, November 24, 2011. http://www. archdaily.com/186069/expandable-surface-pavilion-pablo-estebanzamorano/.

Zumthor, Peter. "Kunsthaus Bregenz." ArchDaily, 1997 1989. http://www. archdaily.com/107500/ad-classics-kunsthaus-bregenz-peterzumthor/. 